\title{
The application of oligonucleotide templated chemical reactions to DNA aptamers
}

\author{
by
}

Christopher M. C. Mattice

A thesis submitted to the Faculty of Graduate and Postdoctoral Affairs in partial fulfillment of the requirements for the degree of

Doctor of Philosophy

in

Chemistry

Carleton University

Ottawa, Ontario

(C) 2018, Christopher M. C. Mattice 


\section{Abstract}

The ability of an aptamer to catalyze a chemical reaction under selective conditions presents a novel avenue for the exploration of biosensors and molecular payload delivery. To date, limited research exists for pairing oligonucleotide-templated chemical reactions with the selective nature of DNA aptamers. A system can be designed wherein the conformational change in aptamer structure associated with target binding brings two previously spatially isolated reactants into proximity, thereby catalyzing their reaction through an increase in effective molarity. A rationally designed aptamer-mediated $\mathrm{S}_{\mathrm{N}} 2$ displacement of a sulfonyl-based fluorescence quencher resulted in an effective increase in fluorescence upon mixing of the aptamer with two appropriately modified complementary oligonucleotides. This increased fluorescence could be slowed by the presence of the aptamer target, permitting the development of an aptamer-based sensor for the mycotoxin ochratoxin A. Using this turn-off type sensor system, a linear dynamic range of $100 \mu \mathrm{M}$ to $100 \mathrm{pM}$ ochratoxin A could be detected with a limit of detection of $1.5 \mathrm{pM}$.

Similar aptamer-based sensor systems were also developed that could take advantage of the fluorogenic copper catalyzed azide-alkyne cycloadditions between two labeled probes that could be slowed by the presence of the aptamer target. This has been demonstrated using the thrombin structure switching DNA aptamer to produce a linear response to thrombin between $100 \mathrm{nM}$ and $10 \mathrm{pM}$ with a limit of detection of $3.9 \mathrm{fM}$. These fluorogenic click modifiers were also incorporated directly into a structureswitching thrombin aptamer such that the G-quadruplex formed upon thrombin binding 
brings the two modifiers into proximity to allow the reaction. This constitutes a turn-on sensor for thrombin, with detection of thrombin between $1 \mu \mathrm{M}$ and $1 \mathrm{nM}$ possible.

As a final proof of concept, the fluorogenic azide and alkyne modifiers were incorporated on either side of a split aptamer for cocaine, such that the presence of cocaine will result in a detectable fluorescent signal from ligation of the split aptamer sequences. Using this system, a qualitative cocaine sensor in the concentration range of $100 \mu \mathrm{M}$ to 10 pM was established. This project has demonstrated the first steps toward controlling chemical reactions using DNA aptamers. Having demonstrated this preliminary functionality, future applications in drug delivery, enhanced sensors, and selective chemical synthesis constitute intriguing new avenues for aptamer research. 


\section{Acknowledgements}

First, I would like to extend my deepest gratitude to both of my thesis supervisors, Dr. Maria DeRosa and Dr. Jeff Manthorpe. Maria accepted this shy and unsure undergraduate student into her lab, and through her encouragement and enthusiasm nurtured me into the scientist and presenter that I have become today. Jeff helped me to see past my synthetic organic background- what had been my true passion as an undergradand look at applications of organic chemistry instead, leading me to this wonderful collaborative project. Both of you have allowed me to work independently and grow as a scientist and a person under your amazing guidance- I cannot thank you enough.

Within the Department of Chemistry at Carleton University, I would also like to thank a few individuals for their guidance and help along the way. Dr. Peter Buist gave me my first taste of research as an undergraduate student, and his inspiration and encouragement led to my thirst for research excellence in my Ph.D. Jim Logan singlehandedly saved many of my experiments throughout my Ph.D. that were doomed by the various malfunctioning pieces of equipment- I couldn't have finished without him. Elena Munteanu hired me as a TA in the second year of my undergraduate studies and kept me as a TA all the way through the end of my Ph.D.- I'm going to miss that lab and our chats! Tony O'Neill and Keith Bourque- thanks for the laughs- you always made TAing an absolute blast!

To all members of the DeRosa lab- past and present- thank you for being by my side for an amazing five years of grad school filled with fun memories! You were always there for the celebration when experiments worked, and for the support when experiments didn't. Thank you, and best of luck to all of you in your future. From the Manthorpe lab, 
Monica Gill and Sam Shields were always there to offer their perspective on a reaction and help any way that they could. Thank you both!

I've made a lot of connections through the years, but there are a few people that will always stand out in my mind and need to be thanked for all their support throughout grad school. Will Turnbull, our late-night gaming venting-sessions and constant supportive messaging back and forth throughout grad school helped us both. Best of luck on your next adventure in Edmonton! Dan Goudreau- we may not have been best friends at the beginning, but once I got past that grumpy demeanour I found an awesome guy that I could talk about science, cars, dogs, life, and CrossFit with! I still miss our coffee conversations! John Nelson, you have been my mentor since undergrad. Whenever I needed any encouragement from anyone outside the lab, you were there. You were always there to give me a kick in the butt when I needed it, but also a laugh when you saw that was more fitting. Grad school would have been much different without being able to sneak away for a chat in your office and coming back refreshed and ready to take on the worldthank you so much!

Finally, I must acknowledge my family. Mom and Dad, you encouraged me to follow my dreams of being a scientist and a doctor from a very young age- and look where I am now! I wouldn't be here without you and all the support that you have given me throughout the years- I owe you more than you can imagine. To my sisters, Jessica and Amanda, thanks for keeping me sane and acting as a mini support group of science students in my own home- we all made it! Tucker, though you've only been here for the last few years, you've become my science support pup- thank you for all the support while I was writing my thesis. Grandma, I told you we would do it together, and we did. Now let's go on another adventure. 


\section{Table of Contents}

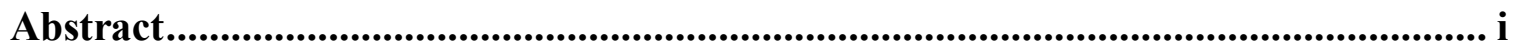

Acknowledgements .........................................................................................................

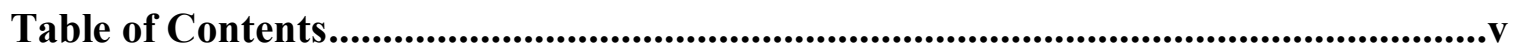

List of Tables .................................................................................................................... xii

List of Figures ....................................................................................................................xv

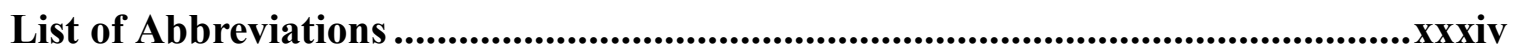

List of Appendices ..............................................................................................................xxxvii

1 Chapter: An introduction to DNA aptamers and Oligonucleotide Templated

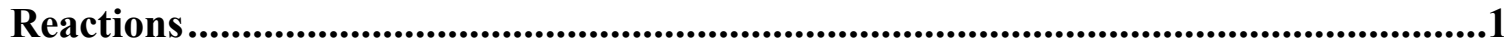

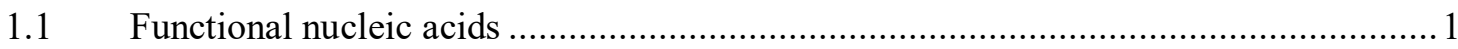

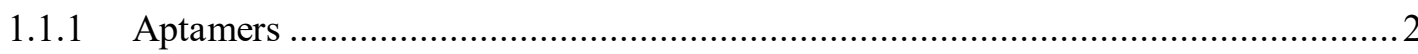

1.1.1.1 Systematic Evolution of Ligands by Exponential Enrichment (SELEX) .........5

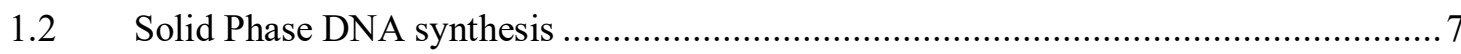

1.2.1 Phosphodiester oligonucleotide synthesis ................................................ 7

1.2.2 Phosphotriester and phosphite-triester chemistry .........................................

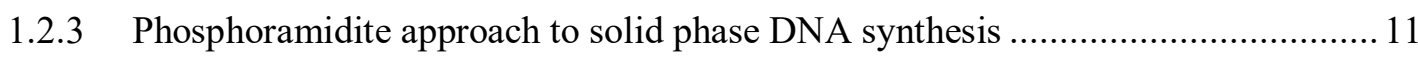

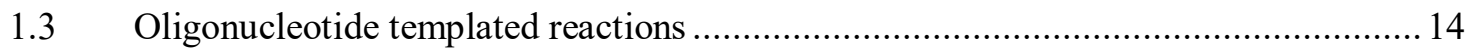

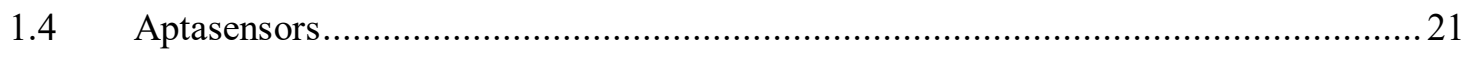

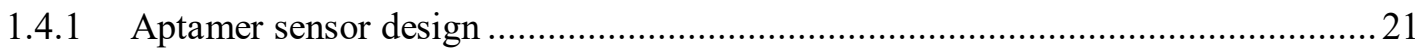

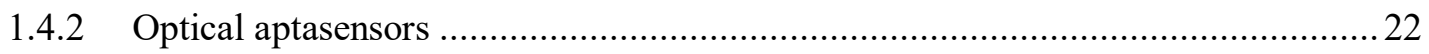

1.5 Designing a DNA aptamer-based sensor using oligonucleotide templated reactions .. 24

\section{Chapter: Designing an Ochratoxin A sensor based on Quenched-Autoligation} probes ................................................................................................................................26

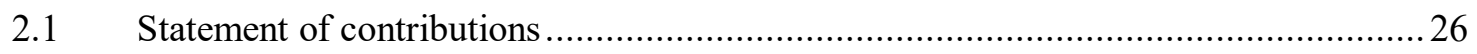




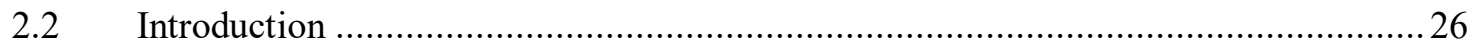

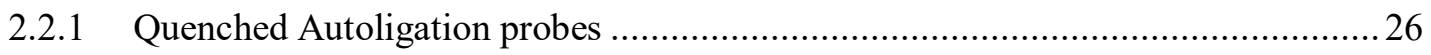

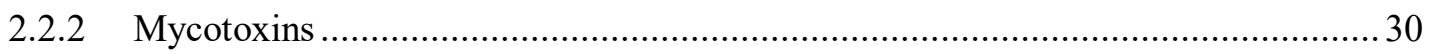

2.2.2.1 An introduction to mycotoxins and their importance ................................. 30

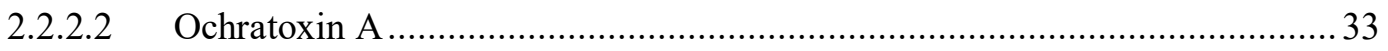

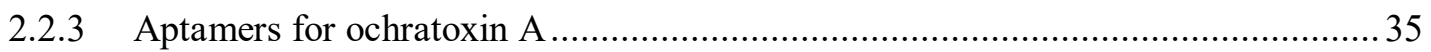

2.2.4 Proposed sensor design for ochratoxin A …...................................................... 36

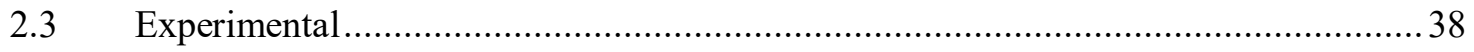

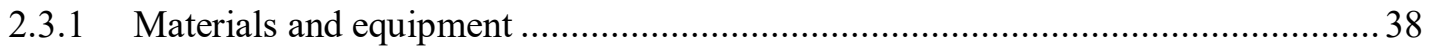

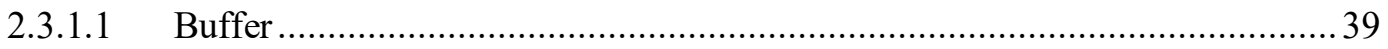

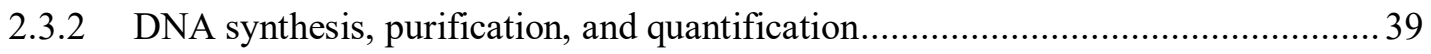

2.3.2.1 Glen Pak DNA purification and desalting.............................................. 41

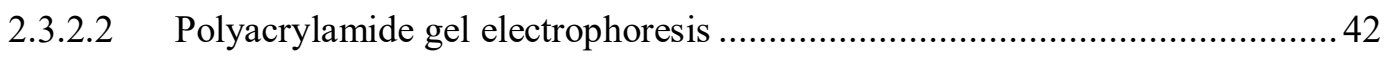

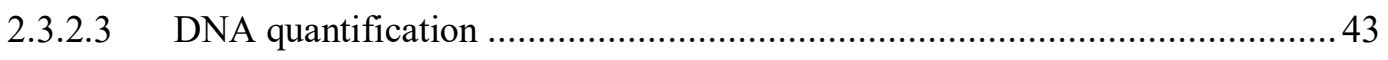

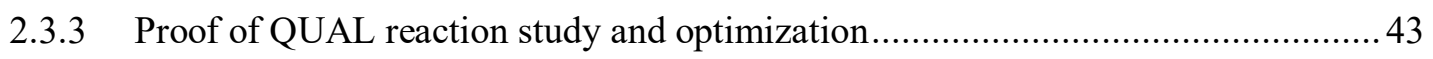

2.3.4 QUAL experiments in the presence of ochratoxin A ....................................... 44

2.3.4.1 General protocol for QUAL experiment with ochratoxin A ........................ 44

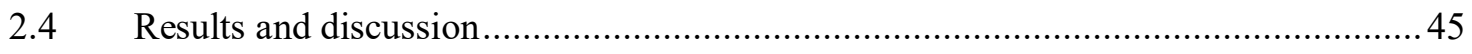

2.4.1 Incorporation of thiophosphate and dabsyl/fluorescein modifiers on DNA sequences 45

2.4.2 Demonstration of OTR functionality in the absence of ochratoxin A

2.4.3 Demonstration of the effect of high levels of ochratoxin A on the measured

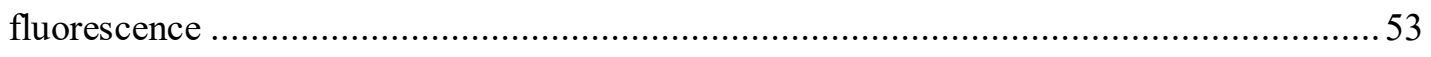

2.4.4 Challenges associated with dabsyl lability and thiophosphate oxidation .................56

2.4.5 Limit of detection and linear dynamic range assessment of ochratoxin A sensor

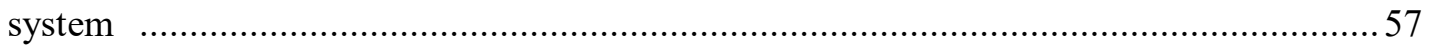


2.5 Conclusions

\section{Chapter: Synthesis of modifiers for fluorogenic click templated chemical} reactions using DNA .................................................................................69

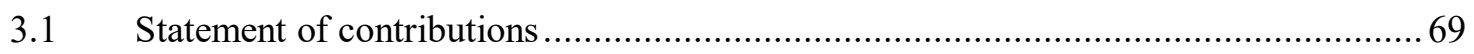

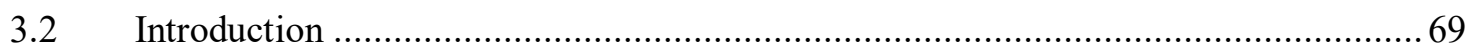

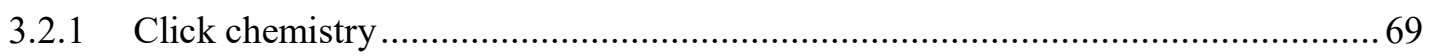

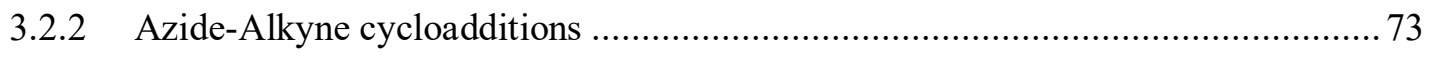

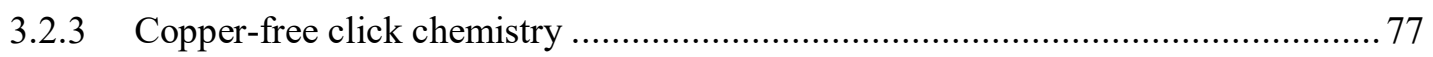

3.2.4 Example of click reactions used in aptamer sensor development ........................ 81

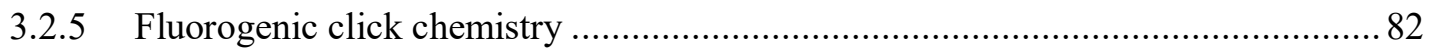

3.2.6 The application of fluorogenic click modifiers to DNA aptasensors ..................... 86

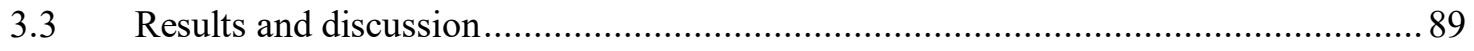

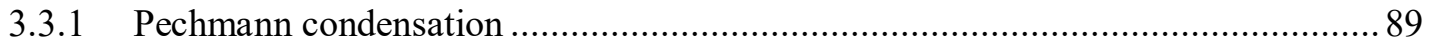

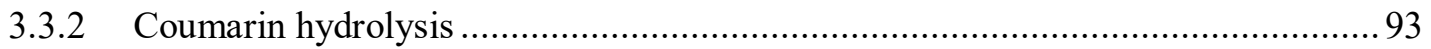

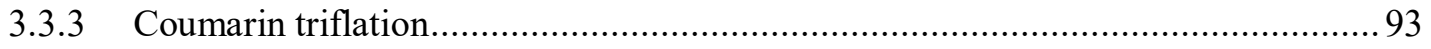

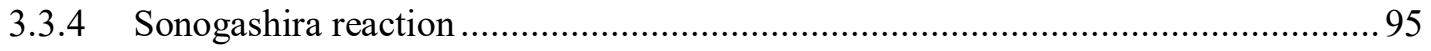

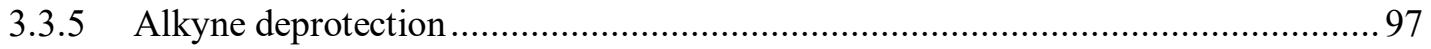

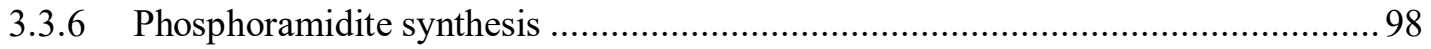

3.3.7 Alkyne-coumarin phosphoramidite synthesis overview................................. 100

3.3.8 Synthesis of 4-azidobutanoic acid and commercial availability ........................ 101

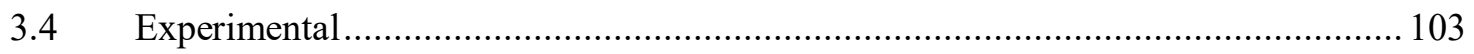

4 Chapter: Fluorogenic Click chemistry for the detection of thrombin ...............110

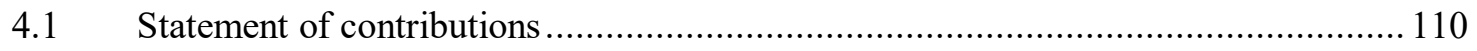

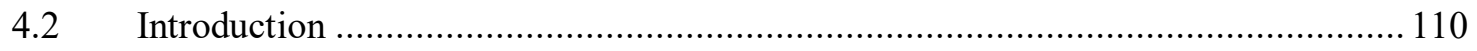

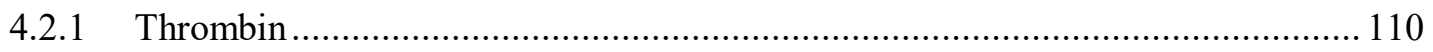




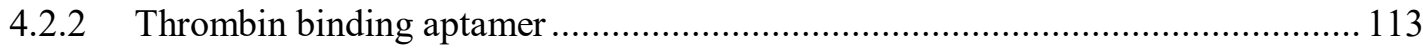

4.2.3 Choice of aptamer for the fluorogenic click reaction sensor ............................ 115

4.2.4 Sensor design rationale for tripartite system................................................. 117

4.2.5 Sensor design rationale for single aptamer system with internal modifiers .......... 118

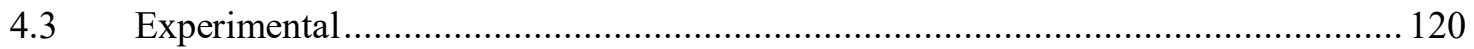

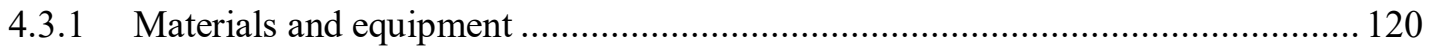

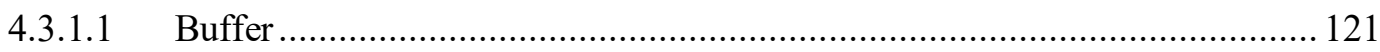

4.3.2 DNA synthesis, purification, and quantification for thrombin tripartite system.... 122

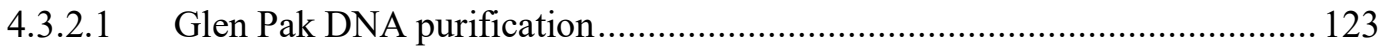

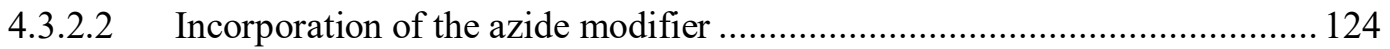

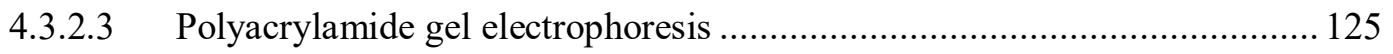

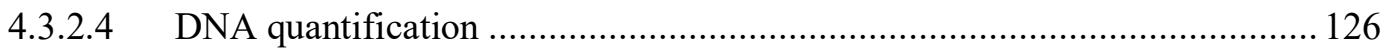

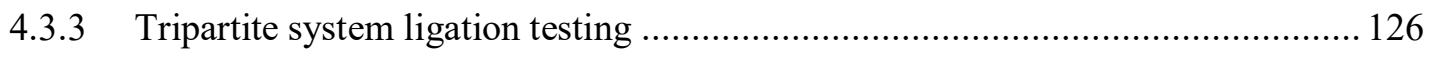

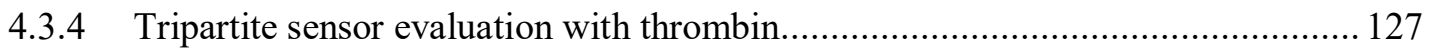

4.3.5 DNA synthesis, purification, and quantification for the thrombin single aptamer

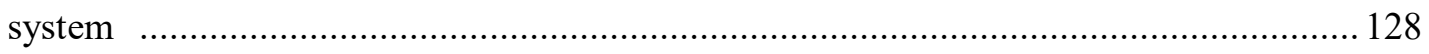

4.3.5.1 Incorporation of azide modifier on thrombin aptamer .............................. 129

4.3.5.2 Incorporation of coumarin modifier on thrombin aptamer ....................... 130

4.3.6 Sensor evaluation for thrombin single aptamer system................................... 131

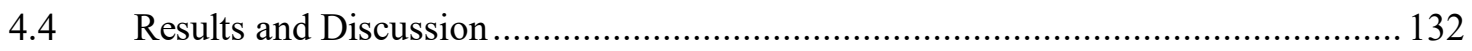

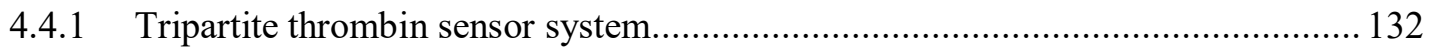

4.4.1.1 Tripartite thrombin capture probe design rationale ................................... 132

4.4.1.1.1 Synthesis and incorporation of alkyne coumarin and azide DNA modifier ...

4.4.1.2 Demonstration of OTR functionality in the absence of thrombin ................ 135

4.4.1.3 Detection of thrombin with the OTR sensor system.................................. 136 
4.4.1.4 Limit of detection and linear dynamic range of click thrombin sensor system...

4.4.1.5 Controls and specificity of thrombin sensor system

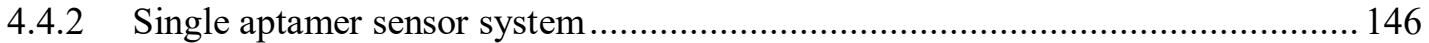

4.4.2.1 Azide and alkyne modification location rationale for single aptamer system146

4.4.2.2 Incorporation of an azide modifier within the SRA 2 aptamer sequence..... 148

4.4.2.3 Incorporation of alkyne coumarin within the SRA_2 aptamer sequence and

associated issues 148

4.4.2.4 Fluorescence signal increase in the presence of thrombin target................. 150

4.4.2.5 Linear dynamic range of single aptamer thrombin sensor system................ 153

4.5 Conclusions

154

\section{Chapter: The detection of cocaine using a split aptamer modified with}

fluorogenic click handles .................................................................................................156

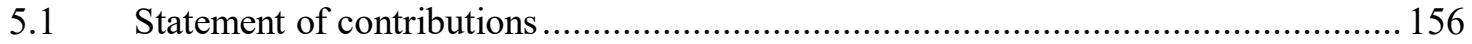

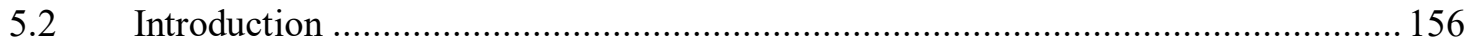

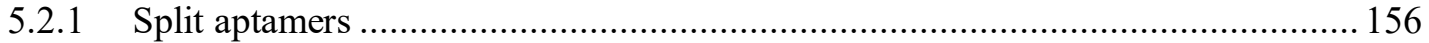

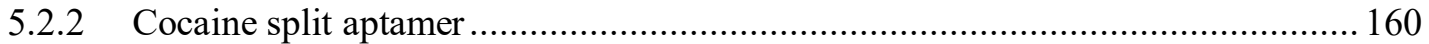

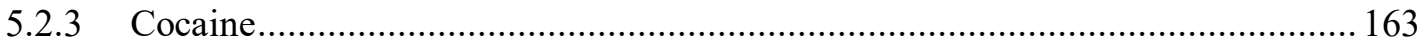

5.2.4 Proposed fluorogenic split aptamer ligation sensor ..................................... 165

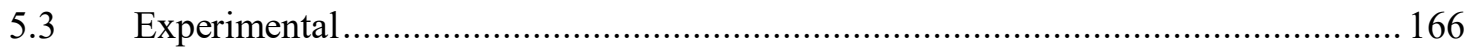

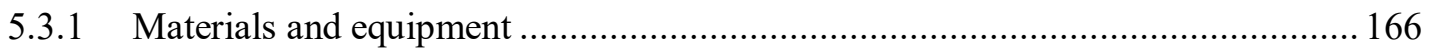

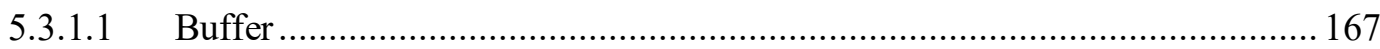

5.3.2 DNA synthesis, purification, and quantification............................................ 168

5.3.2.1 Glen Pak DNA purification.................................................................... 169

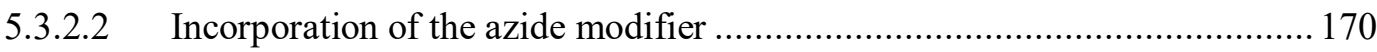

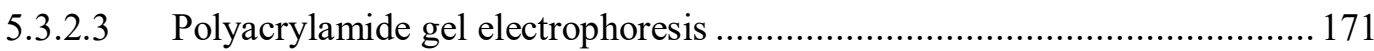




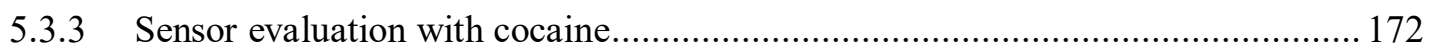

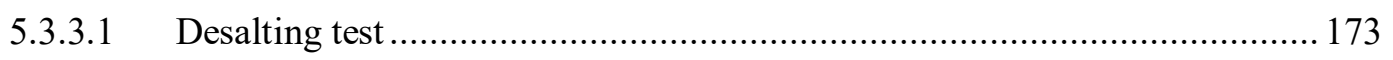

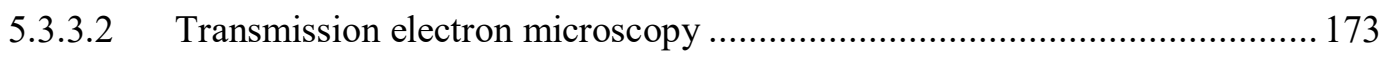

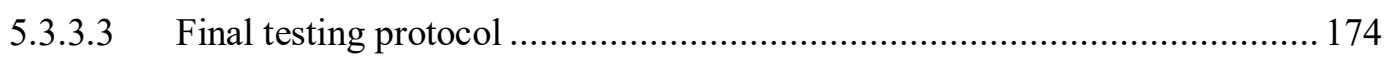

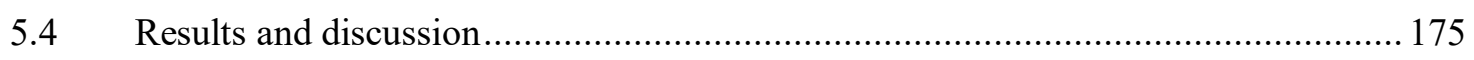

5.4.1 Incorporation of alkyne coumarin into the cocaine split aptamer ....................... 175

5.4.2 Incorporation of the azide modifier into the cocaine split aptamer...................... 176

5.4.3 Challenges associated with fluorescent copper nanoparticles and reaction

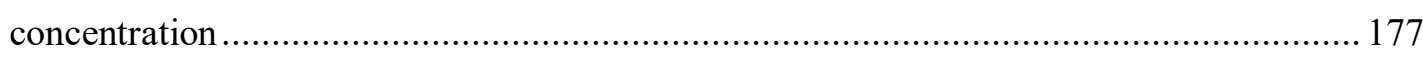

5.4.4 Detection of cocaine with the cocaine split aptamer sensor system.................... 182

5.4.5 Controls and specificity of the cocaine split aptamer sensor system ................... 189

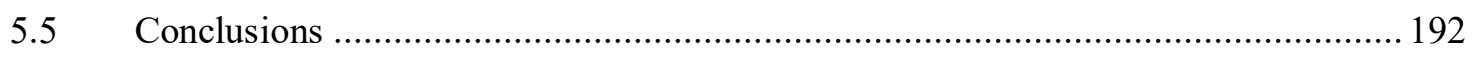

6 Chapter: Significance and future work ...................................................194

Appendices .................................................................................................199

Appendix A : Mass spectra for the Quenched Autoligation sensors ................................. 199

A.1 Deconvoluted mass spectrum of the ochratoxin A aptamer 1.12.2, confirming its

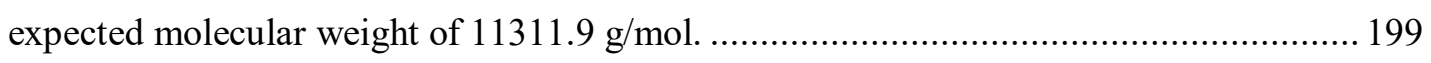

A.2 Deconvoluted mass spectrum of the thiophosphate probe, confirming its expected

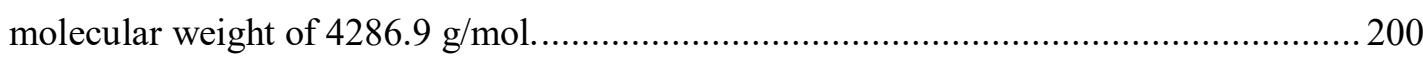

A.3 Deconvoluted mass spectrum of the dabsyl probe, confirming its expected molecular weight of $4943.8 \mathrm{~g} / \mathrm{mol}$ 201

A.4 Deconvoluted mass spectrum of the decomposed dabsyl probe, demonstrating the loss of the dabsyl modifier to give a molecular weight of $4695.9 \mathrm{~g} / \mathrm{mol}$. 201

A.5 Deconvoluted mass spectrum of the dimerized thiophosphate probe. 202 
A.6 Deconvoluted mass spectrum of the ligation product excised from a polyacrylamide gel, showing the ligated product at $8408.4 \mathrm{~g} / \mathrm{mol}$, along with the OTA aptamer 202

Appendix B NMR spectra. 203

B.1 ${ }^{1}$ H-NMR spectrum of 4-(chloromethyl)-7-hydroxy-2H-chromen-2-one 3.2 in d6DMSO. .203

B.2 ${ }^{1}$ H-NMR spectrum of 7-hydroxy-4-(hydroxymethyl)-2H-chromen-2-one 3.3 in d6DMSO. .204

${ }^{1} \mathrm{H}-\mathrm{NMR}$ spectrum of 7-hydroxy-4-(hydroxymethyl)-2H-chromen-2-one 3.4 in $\mathrm{CDCl}_{3}$... 205

B.3 ${ }^{1}$ H-NMR spectrum of 4-(hydroxymethyl)-7-((trimethylsilyl)ethynyl)-2H-chromen-

2-one 3.5 in $\mathrm{CDCl}_{3}$. 206

B.4 ${ }^{1} \mathrm{H}-\mathrm{NMR}$ spectrum of 7-ethynyl-4-(hydroxymethyl)-2H-chromen-2-one 3.6 in d6DMSO. .207

B.5 ${ }^{1} \mathrm{H}-\mathrm{NMR}$ spectrum of 2-cyanoethyl ((7-ethynyl-2-oxo-2H-chromen-4-yl)methyl) diisopropylphosphoramidite 3.7 in $\mathrm{CDCl}_{3}$. 208

Appendix C Mass spectra for thrombin sensors. 209

C.1 Deconvoluted ESI-MS of the 5'-coumarin SRA_2 probe ..................................209

C.2 Deconvoluted ESI-MS of the 3'-amine SRA_2 probe .......................................209

C.3 Deconvoluted ESI-MS of the 3'-azide SRA_2 probe ...................................... 210

C.4 Deconvoluted ESI-MS of the SRA_2 aptamer ............................................... 210

C.5 Deconvoluted ESI-MS of the single aptamer system labeled with the azide and the amine modifiers 211

C.6 Deconvoluted ESI-MS of the single aptamer system labeled with the azide and the coumarin modifiers 211

C.7 Deconvoluted ESI-MS of the SRA_2 capture probe.........................................2 212

Appendix D Mass spectra for cocaine sensor 
D.1 Deconvoluted mass spectrum of the alkyne-coumarin modified cocaine split aptamer, confirming its expected molecular weight of $7680 \mathrm{~g} / \mathrm{mol}$.

D.2 Deconvoluted mass spectrum of the amine modified cocaine split aptamer, confirming its expected molecular weight of $4242 \mathrm{~g} / \mathrm{mol}$

D.3 Deconvoluted mass spectrum of azide modified cocaine coumarin split aptamer. 214

D.4 Deconvoluted mass spectrum of the cocaine ligation product, with the expected molecular weight of the ligated product being 12030.9

References. .216 


\section{List of Tables}

Table 2.1: DNA sequences of all known ochratoxin A aptamers....................................36

Table 2.2: Buffer composition for ochratoxin A quenched autoligation probe sensor experiments.

Table 2.3: Oligonucleotide sequences used in the QUAL experiment for designing an ochratoxin A sensor. TD is the dabsyl-thymidine modifier, TF is the fluoresceinthymidine modifier, PS is used to indicate the location of the thiophosphate modifier, and PO is a 3'-phosphate modification.

Table 3.1: Reaction conditions and repeated trials for the product of 3.2 with yield indicated.

Table 3.2: Summary of the capricious nature of the Sonogoshira reaction as observed in this experiment. Yield appeared to vary regardless of reaction time, conditions, and freshness of the palladium catalyst .............................................................................97

Table 4.1: Sequence of thrombin binding aptamers developed by Bock and Tasset......114 Table 4.2: Aptamer sequences identified by Oh et al. for bindng to human $\alpha$-thrombin. ${ }^{46}$

Table 4.3: Buffer composition for both the tripartite and single sequence thrombin

fluorogenic sensor projects

Table 4.4: Oligonucleotide sequences used in the fluorogenic thrombin tripartite click experiment. The abbreviation "Coum" is used to indicate the location of the alkyne- 
coumarin DNA modifier, while the N3 label is used to indicate the location of the azide

modifier.

Table 4.5: Oligonucleotide sequences used in the fluorogenic thrombin single aptamer click experiment. The abbreviation "coum" is used to indicate the location of the alkynecoumarin DNA modifier, while the $\mathrm{N}$ label is used to indicate the location of the azide modifier.

Table 4.6: Estimated melting temperatures for each probe based on entering the sequence into IDT OligoAnalyzer.

Table 5.1: A summary of currently published DNA and RNA split aptamers

Table 5.2: Buffer composition for ochratoxin A quenched autoligation probe sensor

experiments.

Table 5.3: Oligonucleotide sequences used in the fluorogenic split aptamer click experiment for designing a cocaine sensor. The abbreviation "coum" is used to indicate the location of the alkyne-coumarin DNA modifier, while the $\mathrm{N}_{3}$ label is used to indicate the location of the azide modifier. 168 


\section{List of Figures}

Figure 1.1: The phosphodiester approach to DNA synthesis, using a 3'-acetylated 5'phosphorylated nucleotide to generate a dinucleotide..................................................

Figure 1.2: Structures of the protected bases developed by Khorana, benzoyl adenine and cytosine, and isobutyryl guanine. The 5'-OH protecting group dimethoxytrityl is also

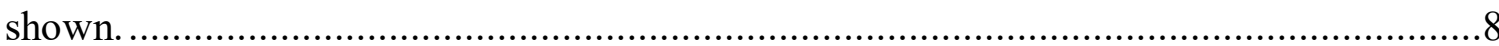

Figure 1.3: The phosphotriester method of DNA synthesis, starting from a $\beta$-cyanoethyl

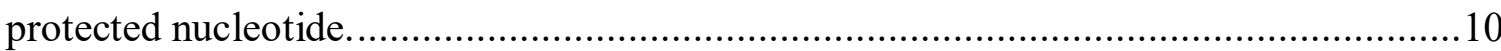

Figure 1.4: The phosphite triester method of DNA synthesis, denoting the synthesis of the phosphochloridite nucleotide, and its reaction with the subsequent base prior to oxidation to the phosphate.

Figure 1.5: The phosphoramidite approach to DNA synthesis results in the production of

a phosphite

Figure 1.6: The oxidation of the phosphite from the previous step in the phosporamidite DNA synthesis method results in the formation of a phosphate

Figure 1.7: First two steps in solid phase DNA synthesis, demonstrating the DMT deprotection of the support-bound nucleotide using trichloracetic acid, followed by 
reaction with the subsequent phosphoramidite in tetrazole to generate the phosphite ester.

Figure 1.8: A capping step is performed using acetic anhydride to consume any unreacted

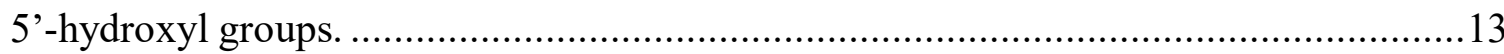

Figure 1.9: Oxidation of the phosphite triester to the phosphate is the final step before another round of detritylation and reaction with the subsequent phosphoramidite. .14 Figure 1.10: Two different strategies are used in literature for oligonucleotide templated reactions. 1) End labelling of two probes complementary to a third template sequence 2) End labelling of two complementary probes. .15

Figure 1.11: A) Henry reaction between a nitroalkane linked DNA sequence and an aldehyde-linked template in the presence of a complementary sequence. B) Nitro- 
Michael reaction between a nitroalkane linked DNA sequence and a maleimide linked sequence in the presence of a complementary sequence. ${ }^{69}$

Figure 1.12: Wittig olefination DNA templated reaction between a stabilized phosphorous ylide modified DNA and an aldehyde linked DNA probe at $55^{\circ} \mathrm{C}$ in the presence of a complementary sequence demonstrated by Liu et al. at $93 \%$ yield. ${ }^{69} \ldots \ldots . .16$ Figure 1.13: Bis-click ligation of two terminal alkyne-modified DNA sequences in the presence of a complementary sequence using a bifunctional azide as demonstrated by Yang and Seela. ${ }^{72}$

Figure 1.14: Heck coupling DNA templated reaction between aryl iodide modified DNA and acrylamide DNA in the presence of $\mathrm{Na}_{2} \mathrm{PdCl}_{4}$ and a complementary sequence demonstrated by Liu et al. with $26 \%$ yield. ${ }^{69}$ 17 Figure 1.15: Staudinger ligation between an azide labeled DNA sequence and a phosphine labeled sequence when bound to a third complementary sequence to generate 
amine modified DNA and phosphine oxide modified DNA as demonstrated by the Liu lab. ${ }^{74}$

Figure 1.16: The templated staudinger ligation reaction between an azide modified DNA sequence and a triphenylphosphine-fluorescein linked DNA sequence (quenched), to generate fluorescent DNA as demonstrated by Cai et al. ${ }^{76}$

Figure 1.17: The DNA templated unmasking reaction of 7-azidocoumarin (quenched) to generate a coumarin fluorphore through a Staudinger reaction with triphenylphosphine labeled DNA. 19

Figure 1.18: Q-STAR OTR system proposed by Farzini and Kool, wherein the Staudinger reaction and subsequent linker cleavage results in the release of a quencher and restoration of fluorescence of the attached fluorophore. ${ }^{79}$ .20 Figure 2.1: Examples and general structures of each of the most common mycotoxin classes. 32

Figure 2.2: The structures of the most common ochratoxins, with ochratoxin A being the most prevalent and the most toxic. .34

Figure 2.3: A) Catalyzing a quenched autoligation reaction using an ochratoxin DNA aptamer A (light blue), complementary to two probes. One labeled with dabsyl quencher (D) and fluorescein (F), and the other labeled with a 3'-nucleophilic phorphorothioate. B) Addition of ochratoxin A results in aptamer binding, which inhibits the autoligation reaction. This is a simplified representation of the system; dabsyl and fluorescein modifiers are separated by a single base in this and all subsequent figures. .37

Figure 2.4: Representation of the ligation reaction between a dabsyl-quencher labeled probe (D) and a phosphorothioate labeled probe (T, labeled with PS), complementary to 
the ochratoxin A aptamer (light blue, O). The circled bands in the DTO lanes indicate the ligated species. The identity of the bands in the gel is indicated by the labels on the left indicating their location in the gel.

Figure 2.5: Representation of the disabled reaction between a dabsyl-quencher labeled probe (D) and a phosphate labeled probe (P, labeled with PO), complementary to the ochratoxin A aptamer (light blue, O). In the DPO lane no new ligated product is observed. The identity of the bands in the gel is indicated by the labels on the left indicating their location in the gel.

Figure 2.6: Representation of the disabled ligation reaction between a dabsyl-quencher labeled probe (D) and an internal phosphorothioate labeled probe (I, labeled with internal PS), complementary to the ochratoxin A aptamer (light blue, O). In the DIO lane, no ligation product is observed. The identity of the bands in the gel is indicated by the labels on the left indicating their location in the gel. .51

Figure 2.7: Representation of the ligation reaction between a dabsyl-quencher and fluorescein labeled probe (DF) and a phosphorothioate labeled probe (T, labeled with PS), complementary to the ochratoxin A aptamer (light blue, O). The left gel is imaged under UV-light, while the right gel is imaged using a fluorescent lamp. In the presence of the dual modified dabsyl oligo, a new ligated product is observed as indicated by the square outline. This newly ligated product is highly fluorescent as would be expected from quencher loss. The identity of the bands in the gel is indicated by the labels on the left indicating their location in the gel. .52

Figure 2.8: Fluorescence spectrum of the ligation reaction between the dabsyl-fluorescein labeled probe (D), thiophosphate probe $(\mathrm{T})$, and the complementary OTA aptamer $(\mathrm{O})$, in 
the presence and absence of $1 \mathrm{mM}$ OTA concentration. $\lambda_{\mathrm{ex}}=494 \mathrm{~nm}, \lambda_{\mathrm{em}}=500-600 \mathrm{~nm}$. In the presence of all three sequences (DTO) the fluorescence intensity of the reaction is much greater than when ochratoxin $\mathrm{A}$ at $1 \mathrm{mM}$ concentration is added to the reaction (DTO + toxin) .54

Figure 2.9: Bar graph of the data represented in Figure 2.8 at $520 \mathrm{~nm}$ emission demonstrating the ligation reaction between the dabsyl-fluorescein labeled probe (D), thiophosphate probe $(\mathrm{T})$, and the complementary OTA aptamer $(\mathrm{O})$, in the presence and absence of $1 \mathrm{mM}$ OTA concentration. The fluorescence intensity of the reaction at 520 $\mathrm{nm}$ (DTO) is far greater than the comparable background, with the addition of toxin (DTO + Toxin) falling in between these two values as expected.

Figure 2.10: Fluorescence spectrum of the ligation reaction between the dabsylfluorescein labeled probe (D), thiophosphate probe (T), and the complementary OTA aptamer $(\mathrm{O})$, in the presence and absence of a range of ochratoxin A toxin concentrations $(100 \mu \mathrm{M}-1 \mathrm{pM}) . \lambda_{\mathrm{ex}}=494 \mathrm{~nm}, \lambda_{\mathrm{em}}=500-600 \mathrm{~nm}$. The reaction $(\mathrm{DTO})$ results in the highest fluorescence intensity, with the samples containing toxin falling below that in a trend of decreasing fluorescence with increasing ochratoxin A toxin concentration.........58 Figure 2.11: Bar graph of the data $(\mathrm{N}=3)$ represented in Figure 2.10 at $520 \mathrm{~nm}$ emission demonstrating the ligation reaction between the dabsyl-fluorescein labeled probe (D), thiophosphate probe $(\mathrm{T})$, and the complementary OTA aptamer $(\mathrm{O})$, in the presence and absence of a range of ochratoxin A toxin concentrations $(100 \mu \mathrm{M}-1 \mathrm{pM})$. A linear trend in the fluorescence intensity of the reaction in the presence of toxin is observable between $100 \mu \mathrm{M}$ and $100 \mathrm{pM}$ after which is plateaus, with all reactions in the presence of toxin having lower fluorescence output than the standard reaction (DTO). One way subjects 
ANOVA at $\alpha=0.05$ showed a significant difference between all means $[F(16,33)=$ 6074.4, $\mathrm{p}=0]$

Figure 2.12: Fluorescence emission spectrum of dabsyl probe incubated with and without ochratoxin $\mathrm{A}$ at $1 \mu \mathrm{M}$ concentration. The addition of toxin appears to quench the fluorescence emission of the dabsyl probe and result in a slight blueshift of the peak.....61 Figure 2.13: Scatter plot of the observed trend at $520 \mathrm{~nm}$ emission for the reaction in the presence of $100 \mu \mathrm{M}$ to $1 \mathrm{pM}$ ochratoxin A concentrations. A linear trend in fluorescence intensity with toxin concentration is observed between $100 \mu \mathrm{M}$ and $100 \mathrm{pM}$, with an $\mathrm{R}^{2}$ value of 0.989. All data points were run in triplicate $(\mathrm{N}=3)$, with error bars for some concentrations hidden by the markers.

Figure 2.14: Plot of change in fluorescence from DTO for each ochratoxin A concentration, plotted against the logarithm of ochratoxin A concentration in pM. Using the regression analysis, an LOD of $1.5 \mathrm{pM}$ was calculated. All data were run in triplicate $(\mathrm{N}=3)$, with some error bars hidden by markers.

Figure 2.15: Fluorescence spectrum of the ligation reaction between the dabsylfluorescein labeled probe (D), thiophosphate probe (T), and the complementary OTA aptamer $(\mathrm{O})$, in the presence of $10 \mu \mathrm{M}$ concentration of adenosine, ATP, and thrombin as controls. The OTA aptamer was also replaced with the thrombin SRA_2 aptamer. $\lambda_{\mathrm{ex}}=$ $494 \mathrm{~nm}, \lambda_{\mathrm{em}}=500-600 \mathrm{~nm}$. The addition of thrombin and adenosine results in a slight decrease in fluorescence emission in the DTO system, while replacement with the SRA_2 aptamer results in no signal difference from background dabsyl (D). .66

Figure 2.16: Bar graph of the data represented in Figure 2.15 demonstrating the ligation reaction between the dabsyl-fluorescein labeled probe (D), thiophosphate probe (T), and 
the complementary OTA aptamer $(\mathrm{O})$, in the presence $10 \mu \mathrm{M}$ concentration of adenosine, ATP, and thrombin as controls. The OTA aptamer was also replaced with the thrombin SRA_2 aptamer. All data were run in triplicate $(\mathrm{N}=3)$.

Figure 3.1: General reaction scheme for nucleophilic ring opening reactions as seen in click chemistry.

Figure 3.2: General reaction schemes for the addition to carbon-carbon multiple bond class of click reactions. Examples of these reactions include a) addition across an alkene to form a strain three member ring system, b) thiol addition under radical conditions, and c) thiol addition to a Michael acceptor.

Figure 3.3: General scheme of the non-aldol carbonyl chemistry reaction class of click chemistry, depicting the formation of either oximes $X=O$, or hydrazides $X=N R$. Figure 3.4: Cycloadditions occupy an important spot in click chemistry, with the two most prevalent examples being the a) Diels-Alder cycloaddition between an electron rich 
diene and electron poor dienophile to generate a six-membered ring, and b) the azidealkyne cycloaddition to produce a five-membered ring triazole. .73

Figure 3.5: Uncatalyzed thermal 1,3-cycloadditon between an azide and an alkyne results in a $1: 1$ ratio of 1,4-:1,5-substituted triazole. .74

Figure 3.6: a) The copper(I) catalyzed azide-alkyne cycloaddition results in the rapid production of solely the 1,4-triazole regioisomer. b) The triazole has similar $\mathrm{R}_{1}$ to $\mathrm{R}_{2}$ distance to an amide bond.

Figure 3.7: Proposed scheme for the copper-catlyzed azide-alkyne cycloaddition. Two possible transition states are possible, as depicted by A and B, involving either two or three copper atoms respectively.

Figure 3.8: Strictures of the common catalytic triazoles used in copper catalyzed azidealkyne cycloadditions, showing tris[(1-benzyl-1H-1,2,3-triazol-4-yl)methyl]amine (TBTA), and its water soluble analog tris(3-hydroxypropyltriazolylmethyl)amine. .77

Figure 3.9: The strain promoted azide-alkyne cycloaddition occurs between a strained cyclootyne ring and an azide to produce a triazole in the absence of a copper catalyst....78 Figure 3.10: Structures of the activated cyclooctynes produced by the Bertozzi lab, a monofluoromethylene cyclooctyne (MOFO), and a difluoromethylene cyclooctyne (DIFO).

Figure 3.11: Structure of the strained alkyne dibenzocyclooctyne (DIBO), produced by the Boons lab. .80 Figure 3.12: Structure of the biarylazacyclooctyne (BARAC) strained alkyne produced by the Bertozzi lab. 
Figure 3.13: A) Depiction of the cocaine sensor produced by the Heemstra research group, consisting of two labeled cocaine split aptamers brought together by target binding to align the cylooctyne and azide DNA modifiers to permit the strain promoted cycloaddition. B) The strain-promoted azide-alkyne cycloaddition to ligate the two DNA sequences shown in A to generate a triazole. The disadvantage of this system is that gel electrophoresis is not a convenient way to monitor the presence of an analyte, and therefore alternative detection strategies needed to be developed.

Figure 3.14: Scheme of the fluorogenic click reaction developed by Zhou and Fahrini, involving the copper catalyzed azide-alkyne cycloaddition between an alkyne coumarin and benzyl azide. Coumarin numbering convention is shown in red on the structure on the right.

Figure 3.15: Scheme of the fluorogenic click reaction developed by Sivakumar et al. for the reaction between a 3 -azido substitued coumarin and phenyl azide. .83 Figure 3.16: Scheme of the fluorogenic click reaction developed by Wong et al., in which an alkyne modified naphthalimide is reacted with an azide to generate a fluorophore from a profluorophore.

Figure 3.17: 3-azido modified boron-dipyrromethene (BODIPY) dyes act as proflorophores, activated to highly fluorescent molecules upon azide-alkyne cycloaddition with an alkyne. BODIPY numbering convention is indicated in red on the non-fluorescent structure. .85

Figure 3.18: A) Sensor design of Sun and Peng, based on the detection of a target sequence (yellow) through a fluorogenic copper catalyzed azide-alkyne cycloaddition between an alkyne-coumarin and an azide. B) The fluorogenic reaction between the 7- 
alkyne coumarin profluorophore labeled DNA and the azide labeled DNA to generate the fluorescent triazole. .86

Figure 3.19: Overall synthesis scheme for the production of the 7-alkyne coumarin phosphoramidite starting from ethyl acetoacetate, as proposed by Sun and Peng. ${ }^{155}$ .88

Figure 3.20: Overall synthesis scheme for the production of the NHS-activated ester of 4azidobutanoic acid proposed by Sun and Peng. ${ }^{155}$ .88

Figure 3.21: The reaction of ethyl-4-chloroacetoacetate with resorcinol in refluxing toluene using PTSA as reported by Fan et al. proved unsuccessful with no recoverable product. ${ }^{160}$ 91

Figure 3.22: The reaction of ethyl-4-chloroacetoacetate with resorcinol in the presence of sulfuric acid could be run at $0{ }^{\circ} \mathrm{C}$, and resulted in a yield of $71 \%$...... 92 Figure 3.23: The hydrolysis of alkyne coumarin 3.2 in water to produce hydroxycoumarin 3.3 . .93

Figure 3.24: Triflation of the hydroxycoumarin 3.3 using the protocol of Zhou and Fahrni yielded the triflated coumarin 3.4 at $85 \%$ yield. ${ }^{151}$ .95 Figure 3.25: Sonogoshira reaction for the production of TMS-alkyne modified coumarin 3.5 from triflated coumarin 3.4 using the method of Sun and Peng. ${ }^{155}$ .96 Figure 3.26: Deprotection of the TMS-alkyne 3.5 to produce the 7-alkyne-coumarin 3.6 using the protocol of Jeon et al. ${ }^{170}$ .98 Figure 3.27: Production of the final alkyne-coumarin phosphoramidite 3.7 for immediate use on the automated DNA synthesizer. 100 
Figure 3.28: Overall synthetic pathway for the production of alkyne-coumarin 3.6 and alkyne-coumarin phosphoramidite 3.7 with an overall yield of $40.8 \%$ and $22.4 \%$, respectively from ethyl-4-chloroacetoacetate 3.1 .

Figure 3.29: Optimized synthetic pathway proposed by DeForest and Anseth for the production of 4-azidobutanoic acid from methyl-4-bromobutanoate. ${ }^{171}$

Figure 3.30: Structure of the commercially available azidobutryate NHS ester from Glen Research for the modification of DNA, thereby eliminating the need for an optimized synthetic procedure.

Figure 4.1: A simplified representation of the thrombin cascade for the conversion of prothrombin to its active thrombin form, which in turn cleaves fibrinogen to initiate formation of the fibrin clot.

Figure 4.2: Representation of the common G-quadruplex topologies, including A) parallel G-quadruplex, B) antiparallel G-quadruplex adopting the chair conformation, and C) antiparallel G-quadruplex containing a diagonal loop

Figure 4.3: Representation of the thrombin fluorogenic sensor, with the alkyne-coumarin and azide modifier probes depicted in green and the thrombin SRA_2 aptamer in yellow. In the presence of $\mathrm{Cu}^{1}$ and absence of thrombin, a triazole-coumarin fluorophore will form and give a detectable signal, but as shown on the bottom in the presence of thrombin fluorophore synthesis is slowed.

Figure 4.4: Representation of the single aptamer thrombin sensor, depicting the incorporation of the alkyne-coumarin profluorphore and the azide modfier on each side of the G-quadruplex structure. A) In the absence of target the double modified thrombin aptamer is bound to its capture probe (pink), and the alkyne and azide are kept far apart. 
B) In the presence of human $\alpha$-thrombin and a $\mathrm{Cu}^{\mathrm{I}}$ source, the coumarin-triazole fluorophore will form after the G-quadruplex shape change brings the two modifiers into proximity, indicating the presence of thrombin.

Figure 4.5: The reaction of the alkyne coumarin 3.6 with carbonyl diimidazole (CDI) to produce the CDI-activated alkyne coumarin 3.11 for addition to amine labeled DNA. .130 Figure 4.6: Denaturing 19\% polyacrylamide gel electrophoresis run on $10 \mu \mathrm{M}$ reaction between SRA_2 coumarin (C), SRA_2 azide (A), and SRA_2 aptamer (S) loaded into separate wells. Ligated thrombin product is only observable in lanes containing all three sequences (CAS) and is indicated by the black box. The identity of the bands in the gel is indicated by the label on the left, corresponding to the distance travelled in the gel......136 Figure 4.7: Fluoresence emission spectra measured between 350-500nm with an excitation of $333 \mathrm{~nm}$. Reactions consisted of a mixture of SRA_2 coumarin (C), SRA_2 azide (A) and SRA_2 aptamer (S), in the presence of $\mathrm{Cu}(\mathrm{I})$ with or without the addition

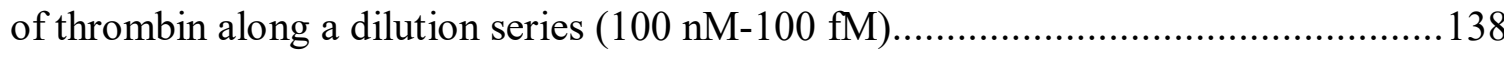
Figure 4.8: Bar plot conversion of the data from Figure 4.7 showing the measured fluorescence intensity at $410 \mathrm{~nm}$ for each of the reactions with the indicated concentration of thrombin. A linear trend in increasing fluorescence intensity with decreasing thrombin concentration is observed between $100 \mathrm{nM}$ and $10 \mathrm{pM}$ thrombin 
concentrations. All data was collected in triplicate $(\mathrm{N}=3)$. One way subjects ANOVA at $\alpha=0.05$ showed a significant difference between all means $[F(16,33)=2682.7, p=0] .140$ Figure 4.9: Scatter plot conversion of the observed trend at $410 \mathrm{~nm}$ emission for the reaction in the presence of $100 \mathrm{nM}$ to $10 \mathrm{pM}$ human $\alpha$-thrombin. A linear dynamic range of $100 \mathrm{nM}-10 \mathrm{pM}$ thrombin is observed.

Figure 4.10: Plot of change in fluorescence from CAS for each thrombin concentration, plotted against the logarithm of thrombin concentration in $\mathrm{pM}$. Using the regression analysis, an LOD of $3.9 \mathrm{fM}$ was calculated. All data was collected in triplicate $(\mathrm{N}=3)$, with error bars appearing smaller than some markers.

Figure 4.11: Fluorescence spectrum of the ligation reaction between the SRA_2 coumarin (C), SRA_2 azide (A), and the complementary SRA_2 aptamer (S), in the presence of 10 $\mu \mathrm{M}$ concentration of adenosine, and ATP as controls. The SRA_2 azide was also replaced with the cocaine azide probe (CAZ). $\lambda_{\mathrm{ex}}=333 \mathrm{~nm}, \lambda_{\mathrm{em}}=350-500 \mathrm{~nm}$. Fluorescence emission of the reaction in the absence of target was higher than in the presence of all controls. Replacing the azide probe with the cocaine azide probe resulted in only background fluorescence emission in the presence and absence of thrombin (CS CAZ 10 Thr and CS CAS respectively).

Figure 4.12: Bar graph of the data represented in Figure 4.11 at $410 \mathrm{~nm}$ demonstrating the ligation reaction SRA_2 coumarin (C), SRA_2 azide (A), and the complementary SRA_2 aptamer (S), in the presence of $10 \mu \mathrm{M}$ concentration of adenosine, and ATP as controls. The SRA_2 azide was also replaced with the cocaine azide probe (CAZ). Fluorescence emission at $410 \mathrm{~nm}$ of the reaction in the absence of target was higher than in the presence of all controls. Replacing the azide probe with the cocaine azide probe 
resulted in only background fluorescence emission in the presence and absence of thrombin (CS CAZ $10 \mathrm{Thr}$ and CS CAS respectively). All data was collected in triplicate $(\mathrm{N}=3)$. 146

Figure 4.13: The SRA_2 aptamer selected by Oh et al. consisted of three distinct regions. A DNAzyme G-quadruplex forming region shown in green, a four thymine base spacer region shown in red, and the randomized regions selected for target binding shown in black.

Figure 4.14: The modification of the SRA_2 thrombin aptamer at the indicated locations would permit the controllable reaction between the alkyne coumarin (red), and the azide (blue). The DNAzyme region is indicated in green. 148

Figure 4.15: Fluoresence emission spectra measured between 350-500nm with an excitation of $333 \mathrm{~nm}$. Reactions consisted of a mixture of the double modified SRA_2 aptamer mixed with its capture probe and the indicated concentration of human $\alpha$ thrombin. No thrombin (0) resulted in the lowest fluorescence emission at $410 \mathrm{~nm}$, with increasing concentrations of thrombin resulting in a positive increase in fluorescence emission.

Figure 4.16: Bar plot conversion of the data from Figure 4.15 showing the measured fluorescence intensity at $410 \mathrm{~nm}$ for each of the reactions with the indicated concentration of thrombin. No thrombin (0) resulted in the lowest fluorescence emission 
at $410 \mathrm{~nm}$, with increasing concentrations of thrombin resulting in a positive increase in fluorescence emission. Data was collected with a single replicate. 152

Figure 4.17: Scatter plot of the observed trend for the reaction in the presence of $1 \mu \mathrm{M}$ to $1 \mathrm{nM}$ human $\alpha$-thrombin concentrations. Data was collected from a single replicate. .. 153 Figure 5.1: a) Originally proposed structure of the cocaine aptamer MNS-4.1 by Stojanovic et al. b) Improved NMR elucidated cocaine aptamer MNS-4.1 structure by Neves et al. showing the tandem GA mismatch and the two nucleotide bulge in the cocaine binding pocket. ${ }^{187,203,206}$ 161

Figure 5.2: Structure of $(R)-(-)$-cocaine, the most addictive stereoconfiguration of cocaine.

Figure 5.3: A) A sensor for cocaine can be designed by labeling the two fragments of a cocaine split aptamer with a fluorogenic azide-alkyne pair that will only be able to react in the presence of cocaine. B) The fluorogenic azide-alkyne cycloaddition occurs between an alkyne-coumarin profluorophore and an azide to generate a fluorescent coumarin-triazole 166

Figure 5.4: Fluorescence spectra of reaction consisting of $1 \mu \mathrm{M}$ of each of azide labeled and coumarin labeled cocaine split aptamer reacted for one hour in the presence (AC 1000) of $1 \mathrm{mM}$ ochratoxin A and absence of toxin (AC). This initial experiment demonstrated concerns with nanoparticle formation and low reaction concentrations leading to a further investigation of the extraneous peak observed. 179 Figure 5.5: Transmission electron microscope image on a copper grid of the reaction of 1 $\mu \mathrm{M}$ azide and coumarin labeled split aptamer with $1000 \mu \mathrm{M}$ cocaine in the presence of 2 $\mathrm{mM} \mathrm{CuSO}_{4}$ and $4 \mathrm{mM}$ sodium ascorbate. Fluorescent copper nanoparticles in the 8-10 
$\mathrm{nm}$ range were observed from the reaction, confirming the previous suspicions of their presence. 180

Figure 5.6: Assessment of desalting methods to remove the $450 \mathrm{~nm}$ peak assumed to be associated with fluorescent copper nanoparticles. AC stands for azide and coumarin labeled DNA mixed together, while 1000 is $1000 \mu \mathrm{M}$ cocaine. Desalting after the reaction by Glen Pak cartridge, Amicon filter, and ethanol precipitation were explored. Desalting reactions were abandoned after the results of this test, as the $450 \mathrm{~nm}$ contaminant peak could not be removed under any condition, and the loss of the target $410 \mathrm{~nm}$ peak presented downstream sensor application issues due to lack of reproducibility.

Figure 5.7: Polyacrylamide gel (19\%) of the reaction between the cocaine coumarin fragment $(\mathrm{C})$ and the azide cocaine fragment $(\mathrm{Az})$. Cocaine was added to the reactions at the indicated concentrations in $\mu \mathrm{M}$ (AC 1000- AC 1). Lanes labeled 1-1000 are for cocaine alone with no added DNA. A ligated product is observable in the reaction lanes containing cocaine and is indicated by a black box. .183

Figure 5.8: Polyacrylamide gel (19\%) for the reactions in the presence of cocaine (AC $100 \mu \mathrm{M}-\mathrm{AC} 10 \mathrm{pM})$, or absence of cocaine (AC). Lanes C and Az represent the coumarin cocaine fragment, and the azide cocaine fragment respectively. A ligated product band is observable in all lanes containing cocaine with the split aptamer (black box), but not in the lanes missing any of the components. .184

Figure 5.9: Fluoresence emission spectra measured between 350-500nm with an excitation of $333 \mathrm{~nm}$. AC represents the azide and coumarin cocaine fragments mixed together, while $\mathrm{AC} 100 \mu \mathrm{M}$ to $\mathrm{AC} 10 \mathrm{pM}$ represent the reaction in the presence of 
cocaine at the indicated concentration. The reaction in the absence of cocaine (AC) resulted in a similar low signal to the coumarin alone (C), while the addition of cocaine saw a positive increase in fluorescence intensity with cocaine concentration in the reaction system. 186

Figure 5.10: Bar plot conversion of the data from Figure 5.9 showing the measured fluorescence intensity at $410 \mathrm{~nm}$ for each of the reactions. The reaction in the absence of cocaine $(\mathrm{AC})$ resulted in a similar low signal to the coumarin alone $(\mathrm{C})$, while the addition of cocaine saw a positive increase in fluorescence intensity with cocaine concentration in the reaction system. Data was collected in triplicate $(\mathrm{N}=3)$.

Figure 5.11: Fluoresence emission spectra measured between 350-500nm with an excitation of $333 \mathrm{~nm}$. AC represents the azide and coumarin cocaine fragments mixed together, while AC 10 cocaine, thrombin, ATP, and adenosine represent the reaction in the presence of $10 \mu \mathrm{M}$ of the indicated target. SRA_2Az represents reactions where the thrombin azide probe is used in place of the cocaine azide fragment. The reaction in the presence of $10 \mu \mathrm{M}$ cocaine resulted in a large fluorescent signal, while the control reactions all resulted in a low signal comparable to coumarin alone. Replacement of the azide sequence with the thrombin azide resulted in a low signal as well, indicative of the importance of the complementary cocaine azide split aptamer sequence. 190

Figure 5.12: Bar plot conversion of the data from Figure 5.11 showing the measured fluorescence intensity at $410 \mathrm{~nm}$ for each of the reactions. The reaction in the presence of $10 \mu \mathrm{M}$ cocaine resulted in a large fluorescent signal, while the control reactions all resulted in a low signal comparable to coumarin alone. Replacement of the azide sequence with the thrombin azide resulted in a low signal as well, indicative of the 
importance of the complementary cocaine azide split aptamer sequence. Data was

collected from a single replicate..................................................................... 191 


\section{List of Abbreviations}

APS: ammonium persulfate

ATP: adenosine triphosphate

AuNP: gold nanoparticle

BARAC: biarylazocyclooctynone

BEN: Balkan endemic nephropathy

BODIPY: boron dipyrromethene

ACN: acetonitrile

CDI: carbonyl diimidazole

CPG: controlled pore glass

DA: Diels Alder

DCM: dichloromethane

DCVC: dry column vacuum chromatography

DIBO: dibenzocyclooctyne

DIFO: difluorinated cyclooctyne

DIPEA: $N, N$-diisopropylethylamine

DMF: dimethylformamide

DMSO: dimethyl sulfoxide

DMT: dimethoxy trityl

DNA: deoxyribonucleic acid

DON: deoxynivalenol

dT: deoxy-thymidine

DTT: dithiothreitol 
EDTA: ethylenediaminetetraacetic acid

ESI: electrospray ionization

EtOAc: ethyl acetate

FISH: fluorescence in situ hybridization

FRET: Förster resonance energy transfer

LC: liquid chromatography

LDR: linear dynamic range

LOD: limit of detection

LSPR: localized surface plasmon resonance

MeOH: methanol

MOFO: mono-fluorinated cyclooctyne

MS: mass spectroscopy

NHS: $N$-hydroxysuccinimide

NMM: $N$-methylmorpholine

NMR: nuclear magnetic resonance spectroscopy

OTA: ochratoxin A

OTR: oligonucleotide templated reaction

PAGE: polyacrylamide gel electrophoresis

PES: polyethersulfone

PTSA: $p$-toluenesulfonic acid

QCM: quartz crystal microbalance

Q-STAR: quenched Staudinger triggered $\alpha$-azidoether release

QUAL: quenched autoligation 
RNA: ribonucleic acid

RT-PCR: reverse transcription polymerase chain reaction

SAR: structure-activity relationship

SELEX: systematic evolution of ligands by exponential enrichment

SNP: single nucleotide polymorphism

SPR: surface plasmon resonance

TBE: tris-borate EDTA

TBTA: Tris[(1-benzyl-1H-1,2,3-triazol-4-yl)methyl]amine

TCEP: Tris(2-carboxyethyl)phosphine hydrochloride

TEAA: triethylammonium acetate

TEM: transmission electron microscopy

TEMED: $N, N, N^{\prime}, N^{\prime}$-Tetramethylethylenediamine

THF: tetrahydrofuran

THPTA: Tris(3-hydroxypropyltriazolylmethyl)amine

TLC: thin layer chromatography

TMS: trimethylsilyl

UHP: ultra-high purity

UV: ultraviolet 


\section{List of Appendices}

Appendix A Mass spectra for the Quenched Autoligation sensors.................200

Appendix B NMR spectra.............................................204

Appendix C Mass spectra for thrombin sensors............................210

Appendix D Mass spectra for cocaine sensor ..................................214 


\section{Chapter: An introduction to DNA aptamers and Oligonucleotide Templated Reactions}

\subsection{Functional nucleic acids}

Our understanding of the utility of nucleic acids has expanded significantly since the early 1990s. Nucleic acids have broadened their role from being a carrier of genetic information to a building block for numerous applications in nanotechnology and material science and have led to the development of the field of DNA and RNA nanotechnology. ${ }^{1-}$

2 DNA was traditionally viewed as a sequence of complementary hydrogen bonding nitrogenous base pairs that were responsible for carrying genetic information from one cell generation to another, as the molecular basis of inheritance. DNA, being the more stable of the two polynucleotide biopolymers, has experienced far faster growth in applications in nanotechnology especially in those involving the construction of nanostructures and sensor design. ${ }^{1}$

The field of structural DNA nanotechnology has expanded quickly since the initial experiments of Nadrian Seeman in the 1980s. ${ }^{3-4}$ In his initial work, he demonstrated the utility of sequence-complementarity in short DNA sequences to generate structural building blocks with programmable elements to create larger nanostructures through selfassembly. Larger DNA objects could also be prepared through two-dimensional and three-dimensional DNA origami, which was demonstrated in the early $2000 s^{5}$ DNA origami uses short staple strands of DNA to fold a long DNA sequence into a defined structure or shape. ${ }^{6}$ The unique addressability of DNA allows the incorporation of chemical modifiers in defined locations within these DNA nanostructures, permitting an 
expanded range of applications including sensor development and drug delivery. 5,7

Functional DNA nanotechnology has also been broadened past structural applications to include more dynamic applications such as devices that either permit chemical transformations, as DNAzymes, or are capable of binding to targets in a highly selective manner, as aptamers. ${ }^{1}$ DNAzymes are essentially biocatalysts, or enzyme mimics, comprised of DNA and are analogous to the naturally occurring RNA enzymes or ribozymes. ${ }^{8-9}$ DNAzymes have never been discovered in vivo unlike their RNA analogues ribozymes, but are instead selected through an in vitro process from a randomized library over several iterative selection steps. ${ }^{10}$ DNAzymes have been selected for such processes as RNA ligation and cleavage, DNA phosphorylation, and DNA oxidative cleavage. ${ }^{11}$ DNAzymes have also been developed for reactions not acting upon oligonucleotides including those that act as peroxidase analogues and those that are capable of protein backbone and side chain modification. ${ }^{9}{ }^{11-12}$ Applications of DNAzymes include in vivo mRNA degradation and sensor development primarily by taking advantage of the peroxidase or RNA cleavage activity. ${ }^{11}$

\subsubsection{Aptamers}

Aptamers, a term derived from the Latin word "aptus" meaning "to fit", are short sequences of DNA or RNA that are capable of binding to a target with a high degree of affinity and selectivity. ${ }^{13-14}$ These functional oligonucleotides will fold into a distinct three-dimensional structure which has a high affinity to the target against which they are selected. ${ }^{2,15}$ Aptamers were discovered independently by two different research groups in 1990. Andrew Ellington and Jack Szostak selected RNA sequences in vitro that were 
capable of binding to a variety of dyes, while Larry Gold and Craig Tuerk isolated RNA aptamers against bacteriophage T4 in a process they called Systematic Evolution of Ligands by Exponential Enrichment (SELEX). ${ }^{14,16}$ In the same year, Debra Robertson and Gerald Joyce demonstrated the selection of catalytic RNA sequences through a similar selection process starting from a known ribozyme. ${ }^{17}$

Aptamers have been selected against a wide range of targets for a variety of downstream applications. The clear majority of aptamers have been selected against protein targets such as thrombin, followed by small molecules such as mycotoxins. ${ }^{2}, 15,18$ More recently, the therapeutic applications of aptamers have been of interest. resulting in examples of aptamers selected against whole cells and viruses. ${ }^{2,19-24}$ The interactions between aptamers and their targets are based on weak non-covalent interactions, including hydrogen bonding, and van der Waals interactions as well as the hydrophobic effect and stacking interactions. ${ }^{25}$ The affinity of an aptamer for its target is defined as its dissociation constant, $\mathrm{K}_{\mathrm{d}}$, and is typically in the nanomolar to picomolar range. Typically, small molecules have $K_{d}$ values on the higher end of this range (weaker binding) while larger targets like proteins will have values on the lower end of this range (stronger binding). ${ }^{15}$ While low $\mathrm{K}_{\mathrm{d}}$ values and high specificity are one of the defining characteristics of aptamers, some selections do not result in aptamers with both characteristics due to the challenges of the selection process. Typically, selection experiments that favour highaffinity binders will possess lower selectivity, and vice versa, due to the pressures of multiple negative selections (vide infra) potentially excluding species with high binding capacities. ${ }^{19,26}$ A variety of thermodynamically stable three-dimensional structures have 
also been identified for aptamers interacting with their targets, including double helices, imotifs, G-quadruplexes, hairpins, and pseudoknots. ${ }^{27-28}$

Aptamers are commonly compared to antibodies due to the similarities in both their form and function, but it is commonly stated that there are many advantages of aptamers over antibodies. ${ }^{2,15,26}$ One of the those is in the aptamer discovery process occurring in vitro. Since the selection does not rely on animals or cells to generate aptamers, the selection method can be fitted to the downstream application- especially when this involves non-physiological conditions. ${ }^{26,29}$ This also opens the possibility of developing aptamers against targets that would not generate an immune response or could potentially be toxic to the animal used for its production. ${ }^{26}$ Seeing as aptamers are commonly referred to as chemical antibodies, this also infers another distinct advantage. Once an aptamer has been selected and sequenced, that sequence can be replicated on an oligonucleotide synthesizer and purified on demand, thus resulting in minimal batch to batch variation. ${ }^{29}$ This chemical synthesis also permits the inclusion of chemical functionality that is different from the five natural nitrogenous bases, thereby expanding the chemical space of the aptamer and allowing for additional chemistry and potentially higher binding affinity. ${ }^{30}$ Aptamers also have the inherent ability to have their activity modulated through the addition of "antidotes". These antidotes are complementary oligonucleotide sequences that disrupt the aptamer-target binding interaction and are afforded through the natural duplex nature of DNA through Watson and Crick base pairings. ${ }^{31-32}$

Due to their chemical nature, there are numerous applications for aptamersespecially in non-physiological conditions that would not be amenable to antibodies. ${ }^{29}$ The 
primary application of aptamers in the literature is for sensor development for a wide range

of analytes. ${ }^{18,33-36}$ Aptamers have also been used for a variety of therapeutic applications, especially in the field of targeted therapeutics as it applies to personalized medicine and theranostics. ${ }^{37}$ Aptamers would permit the targeted delivery of therapeutics to an individualized disease state, thereby limiting off-target effects associated with most broadacting therapeutics. ${ }^{2}$ 29, 38-39 Though of interest to the scientific community, only one aptamer to date has been approved for therapeutic use, being Pegaptanib, for the treatment of age-related macular degeneration. ${ }^{40-41}$

\subsubsection{Systematic Evolution of Ligands by Exponential Enrichment (SELEX)}

The iterative process for the selection of aptamers is called Systematic Evolution of Ligands by Exponential Enrichment (SELEX). The method for the selection of DNA aptamers begins from a randomized single-stranded library that is synthesized such that an internal randomized region of 30-80 nucleotides in length is flanked by two known primer binding sequences on the $5^{\prime}$ and $3^{\prime}$ ends. ${ }^{15,42}$ Incubation of this library with the target of interest will result in some sequences binding while others do not. Those non-binding sequences are washed away while the binding sequences are kept. The sequences that bind to the target are eluted and amplified by to generate a new pool that is enriched for higher affinity sequences. This process is repeated with the newly enriched library over a series of rounds before the final pool is amplified and sequenced. ${ }^{42}$ After sequencing, the potential aptamers are screened and assessed for target affinity and specificity before the 
best aptamer candidate is chosen. This process is called a positive selection as the target of interest is present. ${ }^{43}$ A negative or counter-selection can also be performed, in which the library is incubated with either the stationary phase if the target is immobilized, and/or with non-targets, and binding sequences are discarded while those that wash off are retained for subsequent steps. ${ }^{43}$

Several different modifications to the selection protocol are possible, as has been detailed in recent reviews. One such amendment is cell-SELEX, which involves incubation of the oligonucleotide pool with a whole cell target bearing the biomarker of interest. ${ }^{22-23,} 44$ Counter selection would involve incubation with non-target cells to eliminate non-specific interactions. The advantage of this process over traditional protein targeted SELEX, is that the cell surface biomarker does not need to be known. Post selection, the cell surface aptamer target can be identified through a pull-down assay. ${ }^{23}$

Another modification on the standard selection protocol is capture SELEX, which is advantageous when selecting aptamers against small molecule targets. Traditionally, the challenge for small molecule selections is the partitioning step between binding sequences and non-binding sequences due to the minimal mass change associated with the binding event when compared to a larger target such as a protein or a whole cell. ${ }^{45}$ To allow for partitioning, the target is typically immobilized on a surface through coupling chemistry, but this presents both steric and chemical limitations for the proper selection of an aptamer. This can also limit downstream applications if the target recognition is limited once it is free in solution. Capture SELEX solves this problem by immobilizing the library instead of the immobilizing the target. ${ }^{15,45}$ The library is designed in such a way that there is a 
central fixed sequence domain flanked by two random sequences and the subsequent primer binding sites. This centrally fixed sequence is designed to hybridize to an immobilized complementary sequence. This hybridization is disrupted upon target binding, permitting the separation of binding from non-binding sequences. This capture SELEX process results in aptamers that are called structure switching aptamers due to the

known structure switching event (from duplex to target complex) upon target binding. ${ }^{45-46}$

\subsection{Solid Phase DNA synthesis}

\subsubsection{Phosphodiester oligonucleotide synthesis}

The initial steps towards viable solid phase DNA synthesis were taken by Khorana between the late 1950s and early 1970s. At this point, the chemical synthesis of DNA was an abstract thought and those attempts that had been made to synthesize oligonucleotides proved unsuccessful based on limited stability of the starting materials. ${ }^{47}$ Khorana is credited with the development of the phosphodiester approach to oligonucleotide synthesis, along with the first publication of the protection groups for the 5'-hydroxyl group as well as the protecting groups for the exocylic amines on the nitrogenous bases.

The phosphodiester approach, as depicted in Figure 1.1, involves the reaction of a 5'-trityl protected nucleoside with a 3'-acetylated 5'-phosphorylated nucleotide to generate a dinucleotide after removal of the protecting groups. ${ }^{48-49}$ Due to the high reactivity afforded by the lack of phosphate protection, branching and side reactions were a detriment to this chemistry resulting in arduous cleanup steps and the eventual abandonment of the chemistry in the early 1970 s. ${ }^{47}$ 


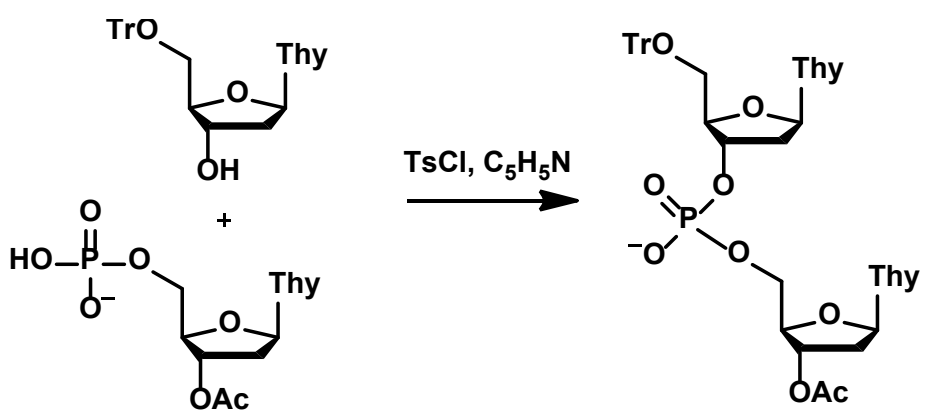

Figure 1.1: The phosphodiester approach to DNA synthesis, using a 3'-acetylated 5'-phosphorylated nucleotide to generate a dinucleotide.

The most important innovation from Khorana's work was the development of protecting groups for the 5'-hydroxyl and the nitrogenous bases. Adenine and cytosine were protected as their benzoyl derivatives, while guanosine was protected as its Nisobutyryl derivative. ${ }^{50}$ Thymine residues remain unprotected. The 5'-hydroxy functionalities on the nucleotides is protected by a trityl leaving group, mainly dimethyoxytrityl protection (DMT). DMT is an acid labile hydroxyl protecting group that can be cleaved rapidly without disturbing the nitrogenous bases. ${ }^{51}$<smiles>[R]n1cnc2c(NC(=O)c3ccccc3)ncnc21</smiles><smiles>[O][R](O)n1ccc(NC(=O)c2ccccc2)nc1=O</smiles><smiles>[R]n1cnc2c(=O)[nH]c(NC(=O)C(C)C)nc21</smiles>

benzoyl cytosine

isobutyryl guanine

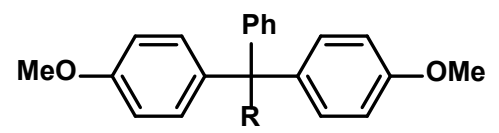

Dimethoxytrityl

Figure 1.2: Structures of the protected bases developed by Khorana, benzoyl adenine and cytosine, and isobutyryl guanine. The 5'-OH protecting group dimethoxytrityl is also shown. 


\subsubsection{Phosphotriester and phosphite-triester chemistry}

The next significant step towards solid phase DNA synthesis occurred in the lab of Letsinger in the 1960s. Letsinger is credited with the first demonstration of solid phase oligonucleotide synthesis, as well as the development of the phosphotriester and phosphite triester methods of DNA synthesis. ${ }^{47}$

In 1965 Letsinger reported on the use of a styrene-divinylbenzene popcorn polymer as a solid polymer support for oligonucleotide synthesis. The proposal was that much like solid phase peptide synthesis, the use of a solid support would simplify the complex stepwise procedure. ${ }^{52}$ This original popcorn polymer suffered from swelling and decomposition over long syntheses and has since been replaced with controlled pore glass (CPG) or crosslinked polystyrene. ${ }^{47}$ The process of solid phase synthesis starts with the 3'-terminal nucleoside residue attached to the solid support, and subsequent residues are added sequentially.

In the phosphotriester method of DNA synthesis, nucleotides are produced containing a $\beta$-cyanoethyl protecting group on the phosphate to protect the internucleotide linkage as in Figure 1.3. ${ }^{52-54}$ This $\beta$-cyanoethyl group is easily removed using ammonium hydroxide during the workup. Stepwise efficiency for the phosphotriester method was rarely above $95 \%$, which plagued long syntheses and limited the utility of the approach. ${ }^{47}$ 


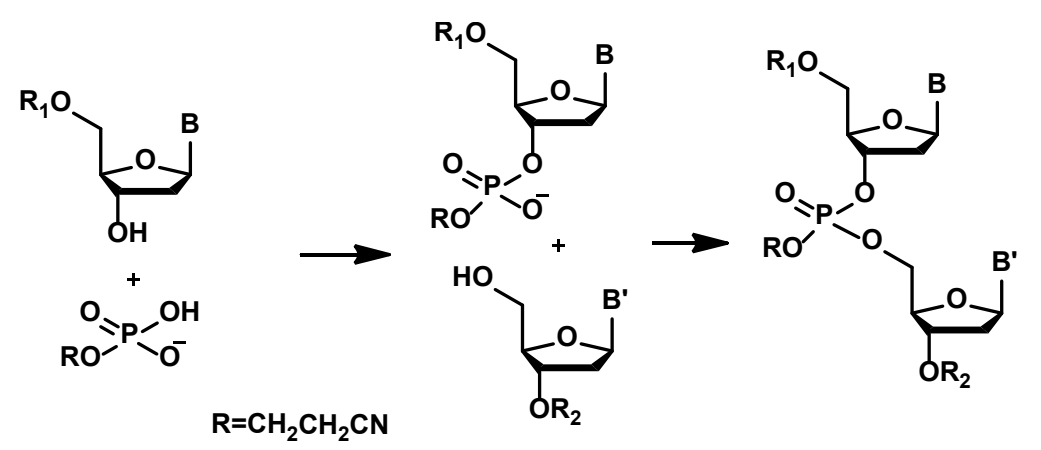

Figure 1.3: The phosphotriester method of DNA synthesis, starting from a $\beta$-cyanoethyl protected nucleotide.

Letsinger and Lunsford discovered in 1976 that P(III) species were significantly more reactive than the corresponding $\mathrm{P}(\mathrm{V})$ acylating agents, prompting the development of the phosphite-triester method of DNA synthesis. ${ }^{55}$ In this method, 5'-protected nucleosides were reacted with 2-chlorophenyl phosphorochloridite to yield a phosphochloridite nucleoside that could readily react with a 3 ' protected nucleoside to yield a dincucleoside phosphite. Treatment in situ with iodine and water resulted in oxidation of the phosphite to the phosphate, yielding the final dinucleotide as shown in Figure 1.4. Letsinger and Lunsford demonstrated this method for the stepwise synthesis of a tetranucleotide. ${ }^{55}$ The advantage of this approach was the increased coupling efficiency and rapid reaction, but the major drawback was the instability of the intermediate phosphorochloridite nucleoside. ${ }^{47}$ Due to their susceptibility to aqueous hydrolysis, these phosphorochloridites had to be prepared immediately before use under arduous $-78^{\circ} \mathrm{C}$ conditions thereby limiting their application to non-chemists. ${ }^{47}$ 

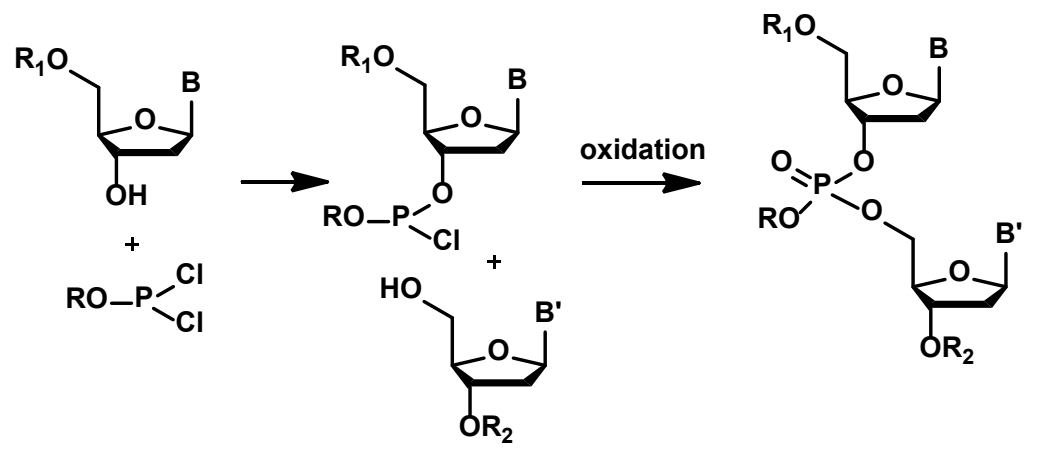

Figure 1.4: The phosphite triester method of DNA synthesis, denoting the synthesis of the phosphochloridite nucleotide, and its reaction with the subsequent base prior to oxidation to the phosphate.

\subsubsection{Phosphoramidite approach to solid phase DNA synthesis}

In 1985, Beaucage and Caruthers published an improvement to the phosphitetriester method of DNA synthesis, wherein the replacement of the chloride leaving group with an amine resulted in a significant change in the properties of the molecule. By reacting 2'-deoxyribonucleosides with chloro(dimethylamino)-methoxyphosphine, they were able to generate the corresponding phosphoramidites. ${ }^{56}$ Phosphoramidites had been shown to react with acids to generate an acyl phosphite, which when combined with an alcohol will form a trialkyl phosphite as shown in Figure 1.5. ${ }^{47}$ These phosphoramidites have the advantage that they could be isolated as a stable solid and stored until needed unlike the previously demonstrated phosphochloridites. ${ }^{47}$ 


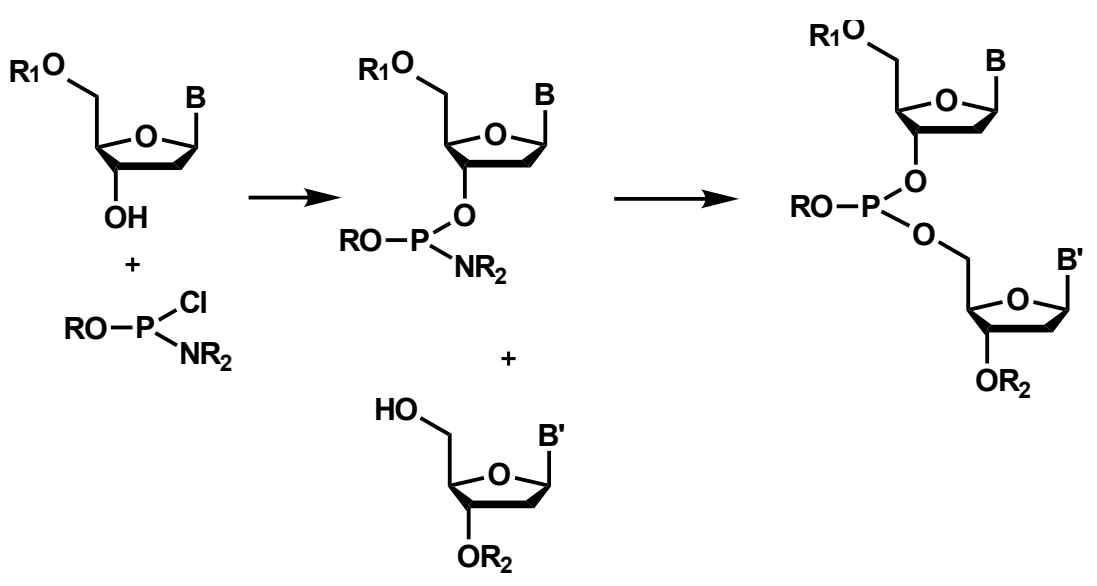

Figure 1.5: The phosphoramidite approach to DNA synthesis results in the production of a phosphite.

As shown in Figure 1.5, reaction of the phosphoramidite with a 3'-protected nucleoside results in the generation of a phosphite just like the phosphite-triester approach, and subsequent oxidation in iodine and water will produce the phosphate linkage. ${ }^{56}$
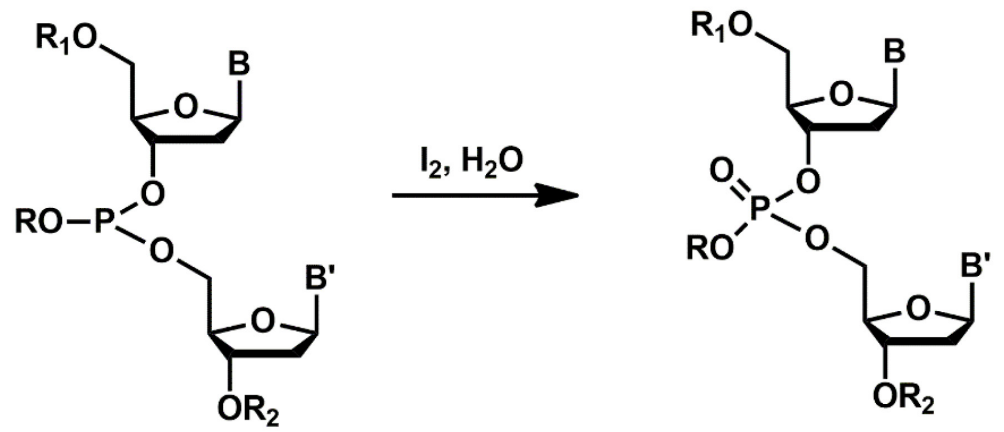

Figure 1.6: The oxidation of the phosphite from the previous step in the phosporamidite DNA synthesis method results in the formation of a phosphate.

The cycle of solid phase DNA synthesis starts from a support-bound 3'-nucleoside having it's 5'-DMT protecting group removed using trichloroacetic acid. This exposes the 5'-OH for reaction with the subsequent phosphoramidite. Addition of the subsequent phosphoramidite along with a tetrazole activator results in replacement of the diisopropylamine leaving group with the 5'-hydroxyl of the support bound nucleoside to 
generate a phosphite triester. ${ }^{47,57}$
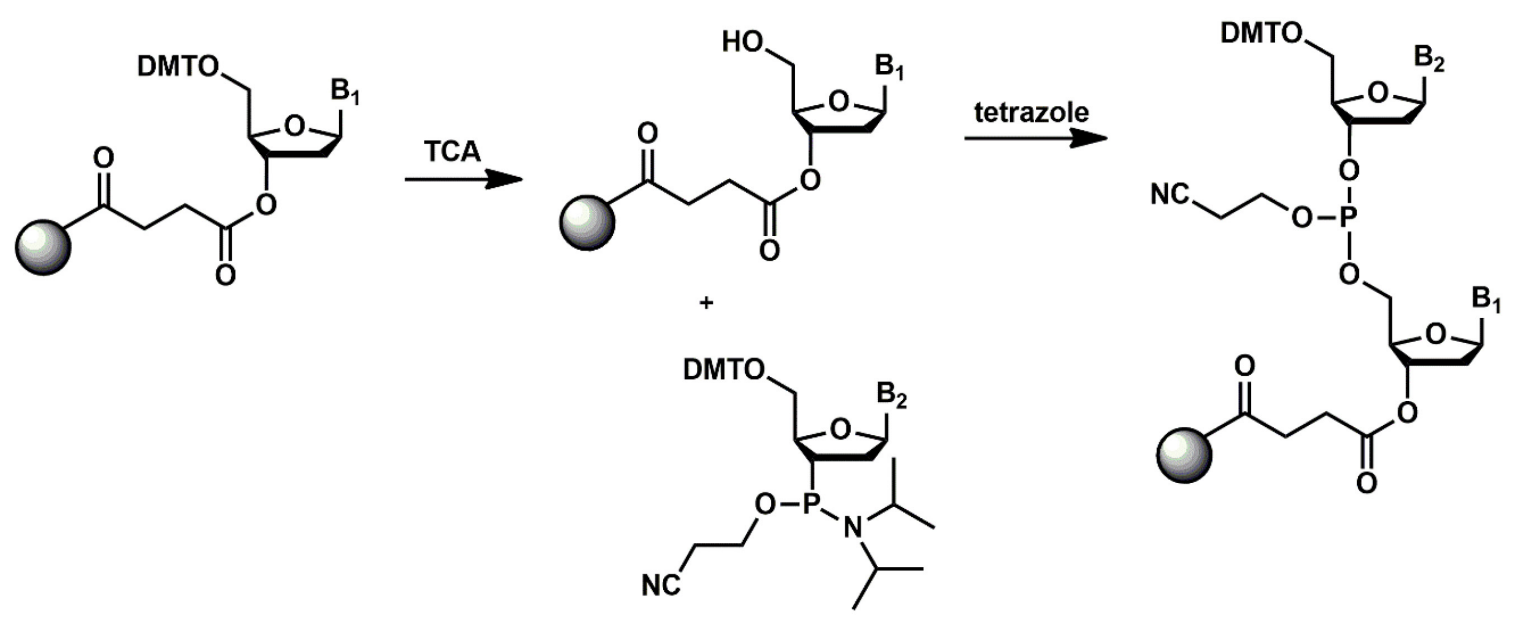

Figure 1.7: First two steps in solid phase DNA synthesis, demonstrating the DMT deprotection of the support-bound nucleotide using trichloracetic acid, followed by reaction with the subsequent phosphoramidite in tetrazole to generate the phosphite ester.

To limit deletion mutations, any non-reacted 5'-hydroxyl groups are capped quantitatively with acetic anhydride in N-methylimidazole. This renders these bases inert to subsequent reactions and eliminates the possibility of any skipped bases during the synthesis cycle. $^{47}$
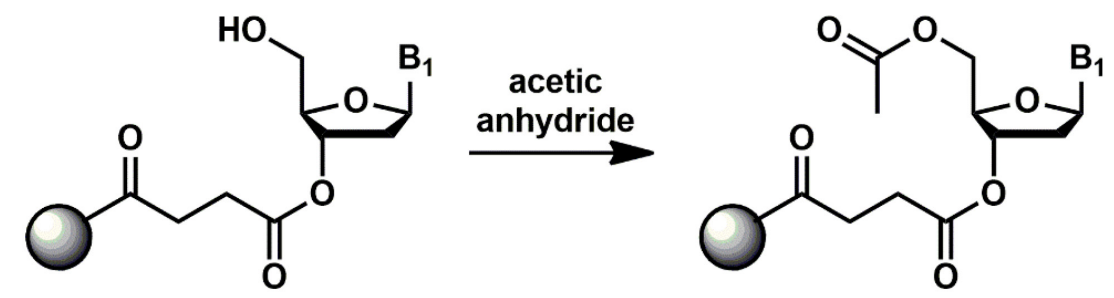

Figure 1.8: A capping step is performed using acetic anhydride to consume any unreacted 5'-hydroxyl groups.

The phosphite triester is then oxidized in the presence of iodine and water from a $\mathrm{P}(\mathrm{III})$ to $\mathrm{P}(\mathrm{V})$, leaving the phosphodiester linkage of natural DNA with the cyanoethyl protecting group intact to prevent side reactions. From here, the synthesis cycle will start 
again with a detritylation step. ${ }^{47,57}$

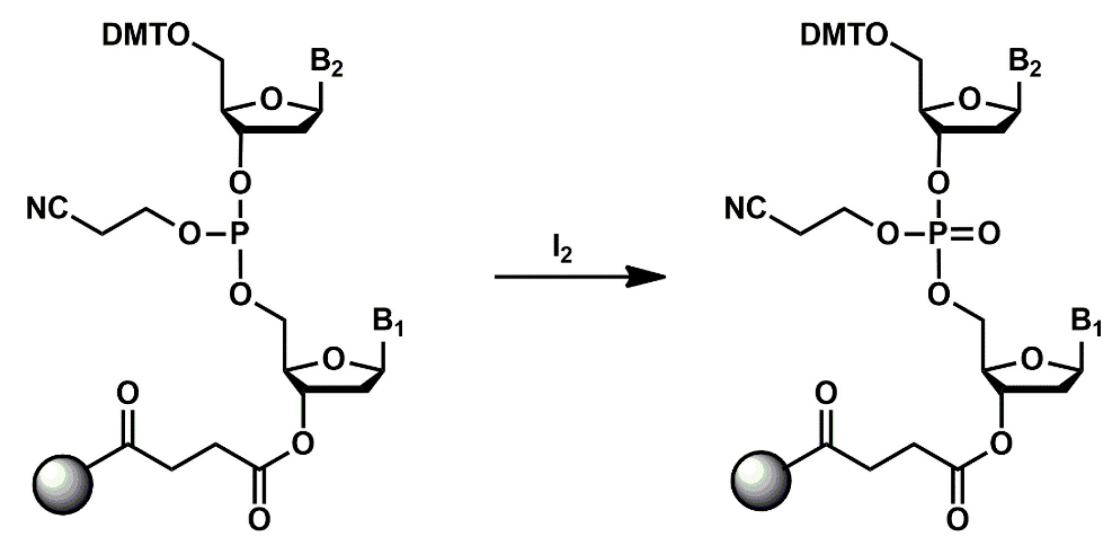

Figure 1.9: Oxidation of the phosphite triester to the phosphate is the final step before another round of detritylation and reaction with the subsequent phosphoramidite.

Following synthesis, the oligonucleotide is cleaved from its succinyl ester linker using ammonium hydroxide. This same solution will also deprotect the nitrogenous bases and remove the cyanoethyl group from the phosphate backbone, leaving the final oligonucleotide in solution. ${ }^{47,57}$

\subsection{Oligonucleotide templated reactions}

Oligonucleotide templated reactions (OTR) can generally be defined as reactions that rely on sequence-specific hybridization to bring together two spatially isolated molecules, enabling the formation of a new chemical bond. ${ }^{58}$ Reactions templated by oligonucleotides are not novel, as there are extensive examples of such reactivity in nature, with DNA replication being perhaps the oldest example of an OTR. ${ }^{59}$ OTR relies on Watson and Crick base pairing to promote the reaction by increasing the effective molarity of two reactants, otherwise present at concentrations too low to favourably evoke a reaction 
in solution. ${ }^{58-59}$ The result is a dramatic increase in reaction rate due to DNA templating. Two strategies exist in literature for OTRs as shown in Figure 1.10, with the first being the end labelling of two sequences complementary to a third template sequence, such that the probe binding the two labeled sequences brings the labels into proximity, and the second being the end labelling of two complementary sequences with the reactive groups. ${ }^{58}$

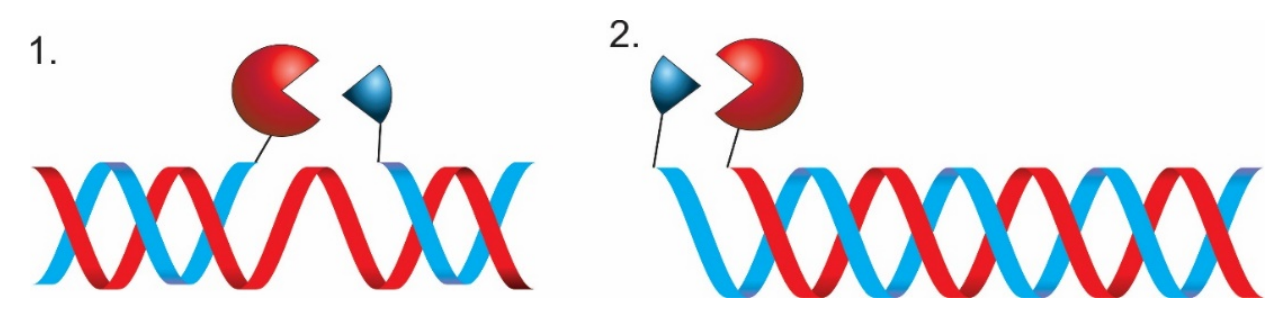

Figure 1.10: Two different strategies are used in literature for oligonucleotide templated reactions. 1) End labelling of two probes complementary to a third template sequence 2) End labelling of two complementary probes.

OTRs can be further subdivided into two categories based on the transformation performed. The first category is ligation reactions, and this consists of any reaction in which the outcome is the ligation of two sequences. ${ }^{58,60}$ The earliest examples were $\mathrm{S}_{\mathrm{N}} 2$ reactions between two oligonucleotides, wherein one oligonucleotide would be functionalized at the 5' end with an electrophilic group, while a second oligonucleotide is labeled at the 3' end with a nucleophile. The Lestinger group initially demonstrated OTR using a bromoacetyl electrophile with a phosphorothioate nucleophile, while the Kool group expanded the chemistry to include iodo and tosyl electrophiles with phosphorothioate and phosphoroselenoate nucleophiles. ${ }^{61-67}$

A wide variety of other chemistry has been applied to oligonucleotide ligation reactions. Lynn et al. demonstrated a reductive amination for ligating sequences 
containing an aldehyde and an amine functionality in the presence of $\mathrm{NaBH}_{3} \mathrm{CN} .{ }^{68}$ Liu et al. demonstrated the first examples of a Henry reaction and a nitro-Michael reaction for ligation of DNA sequences as shown in Figure 1.11. ${ }^{69}$ This was demonstrated through the reaction of nitroalkenes with aldehydes or maleimides in a temperature and $\mathrm{pH}$-dependent fashion. A Wittig olefination was also demonstrated by the Liu group using a stabilized phosphorus ylide and an aldehyde to form an E-olefin linkage between the DNA sequences as in Figure 1.12. ${ }^{69}$

A

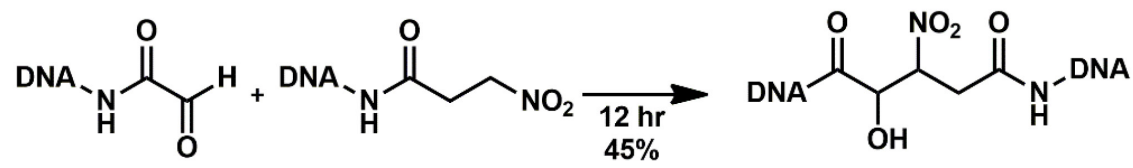

B

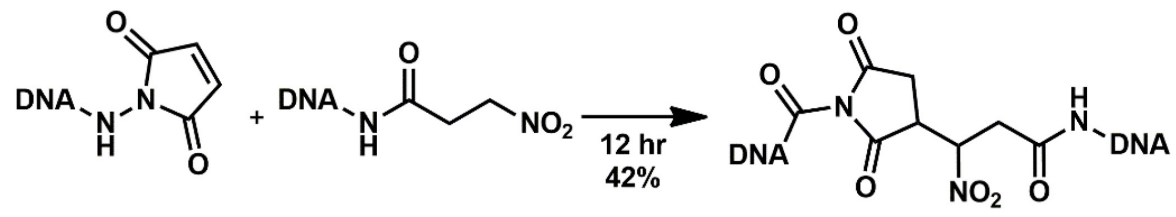

Figure 1.11: A) Henry reaction between a nitroalkane linked DNA sequence and an aldehyde-linked template in the presence of a complementary sequence. B) Nitro-Michael reaction between a nitroalkane linked DNA sequence and a maleimide linked sequence in the presence of a complementary sequence. ${ }^{69}$

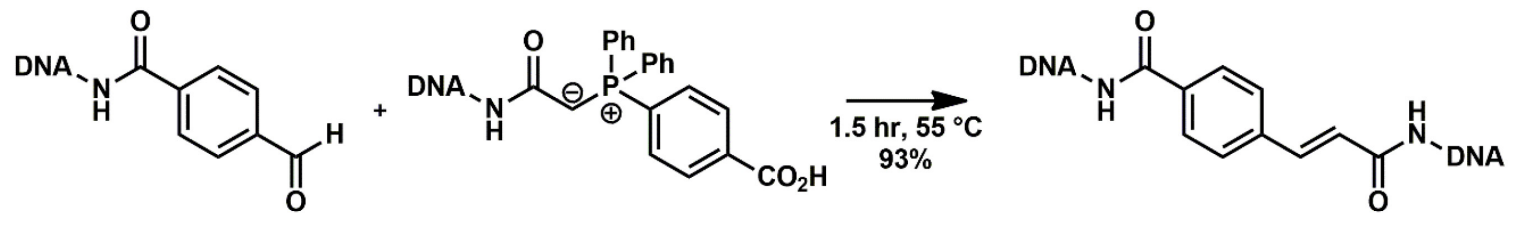

Figure 1.12: Wittig olefination DNA templated reaction between a stabilized phosphorous ylide modified DNA and an aldehyde linked DNA probe at $55{ }^{\circ} \mathrm{C}$ in the presence of a complementary sequence demonstrated by Liu et al. at $93 \%$ yield. ${ }^{69}$

Organometallic reactions have also been used to catalyze OTR ligation reactions, with the most prominent example being the Huisgen cycloaddition. The Huisgen cycloaddition is the copper(I) catalyzed cycloaddition between an azide and an alkyne to 
generate a 1,4-disubstituted triazole. ${ }^{70}$ The first example of a Huisgen cycloaddition applied to OTR was performed by the Liu group for linking a propargyl-glycine and a phenylazide modified sequence in a templated reaction. ${ }^{71}$ In 2017 Yang and Seela demonstrated a bis-click ligation of two terminal alkyne modified DNA probes using a bifunctional azide as in Figure 1.13. ${ }^{72}$ This reaction occurred rapidly in solution in the presence of copper(I) for the ligation of two probes complementary to a template as well as for the circularization of a short DNA sequence end modified with two alkyne groups. Heck coupling has also been demonstrated for the palladium mediated ligation of aryl iodide labeled DNA and alkene bearing DNA (Figure 1.14). ${ }^{71}$

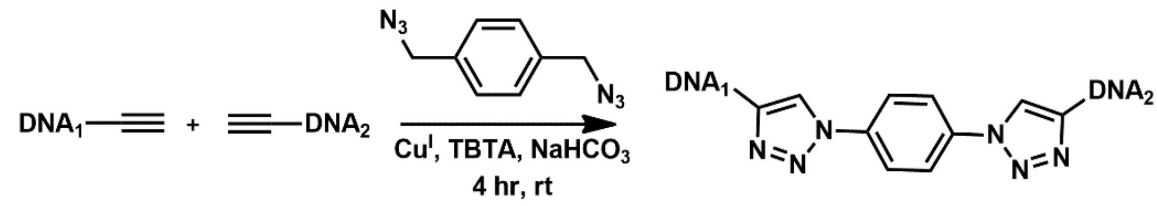

Figure 1.13: Bis-click ligation of two terminal alkyne-modified DNA sequences in the presence of a complementary sequence using a bifunctional azide as demonstrated by Yang and Seela. ${ }^{72}$<smiles></smiles>

Figure 1.14: Heck coupling DNA templated reaction between aryl iodide modified DNA and acrylamide DNA in the presence of $\mathrm{Na}_{2} \mathrm{PdCl}_{4}$ and a complementary sequence demonstrated by Liu et al. with $26 \%$ yield. ${ }^{69}$

OTR ligations are not without their issues, with one of the significant challenges being the increased stability of the product of the reagents preventing reaction turnover and amplification. Due to the increased hydrogen bonding resulting from two ligated strands complementary to the template strand, their affinity for the template strand is higher than 
either of the two starting probes. ${ }^{70}$ This results in a lack of turnover after one reaction cycle, and a variety of methods have been proposed to eliminate this issue with thermocycling being the most prevalent. By running the reaction in a PCR thermocycler, one can encourage probe binding followed by reaction and dissociation of the product in an excess of starting material. ${ }^{70,73}$ Alternative strategies involve the design of reactivity that does not result in ligation of the two probes, thereby eliminating the issue at hand and aiding in template turnover.

The second category of OTR is functional group transformation or templated transformation without ligation. These are reactions that do not result in the ligation of the two oligonucleotide sequences, but instead result in changing the functional group in the attached chemistry on the probe. The most prevalent form of this type of OTR derives from the work of Liu in 2005 in which the Staudinger reaction between a tertiary phosphine and organic azide linked to DNA was performed in a template-directed manner as in Figure $1.15 .^{74}$ The result is not a ligation between the sequences, but the conversion of the organic azide into a primary amine, and the tertiary phosphine into a phosphine oxide. Bertozzi et al demonstrated a modified Staudinger reaction in 2000 in which the aza-ylide intermediate of the reaction is captured by an ester group on the phosphine to generate an amide bond. ${ }^{75}$ Cai et al. demonstrated that by attaching the triphenylphosphine group to a monoalkylated fluorescein via an ester, the fluorescence is quenched, and the reaction between the azide and esterified fluorescein triphenylphosphine will regenerate fluorescence through ester hydrolysis (Figure 1.16). ${ }^{76}$ A similar strategy was undertaken by Pianowski and Winssinger in 2007, and in 2008 by Franzini and Kool, to demonstrate that the masked 
fluorescence of 7-azidocoumarin could be activated in a similar unmasking reaction with triphenylphosphine on DNA as shown in Figure 1.17..$^{77-78}$ This led to the development of systems based on caged fluorophores that could be activated through a simple reaction based on the presence of a DNA template.

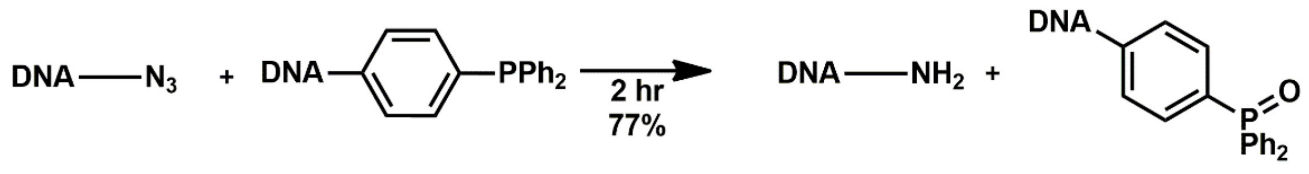

Figure 1.15: Staudinger ligation between an azide labeled DNA sequence and a phosphine labeled sequence when bound to a third complementary sequence to generate amine modified DNA and phosphine oxide modified DNA as demonstrated by the Liu lab. ${ }^{74}$

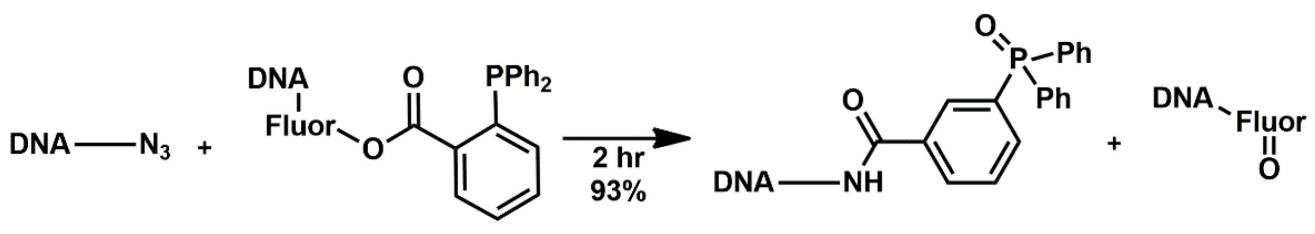

Figure 1.16: The templated staudinger ligation reaction between an azide modified DNA sequence and a triphenylphosphine-fluorescein linked DNA sequence (quenched), to generate fluorescent DNA as demonstrated by Cai et al. $^{76}$<smiles>Cc1c(C(C)C)c(=O)oc2cc([O-])ccc12</smiles>

Figure 1.17: The DNA templated unmasking reaction of 7-azidocoumarin (quenched) to generate a coumarin fluorphore through a Staudinger reaction with triphenylphosphine labeled DNA.

In 2009 Franzini and Kool developed the Q-STAR system of OTR reactions based on the Staudinger reaction. Q-STAR is the quenched Staudinger-triggered $\alpha$-azido-ether release, and consists of a Staudinger reaction resulting in quencher loss eliciting a strong fluorescence signal. ${ }^{79}$ A probe is synthesized containing a fluorophore and a quencher attached through an $\alpha$-azidoether linker. As shown in Figure 1.18, in the presence of 
triphenylphosphine labeled DNA, reduction of the azide is triggered, resulting in linker cleavage and quencher loss. The system was demonstrated using the loss of a dabsyl quencher to induce fluorescein fluorescence. ${ }^{79}$ The reaction was also demonstrated to be compatible with the detection of minute sequence irregularities and to differentiate two different bacterial species both in vivo and in vitro.

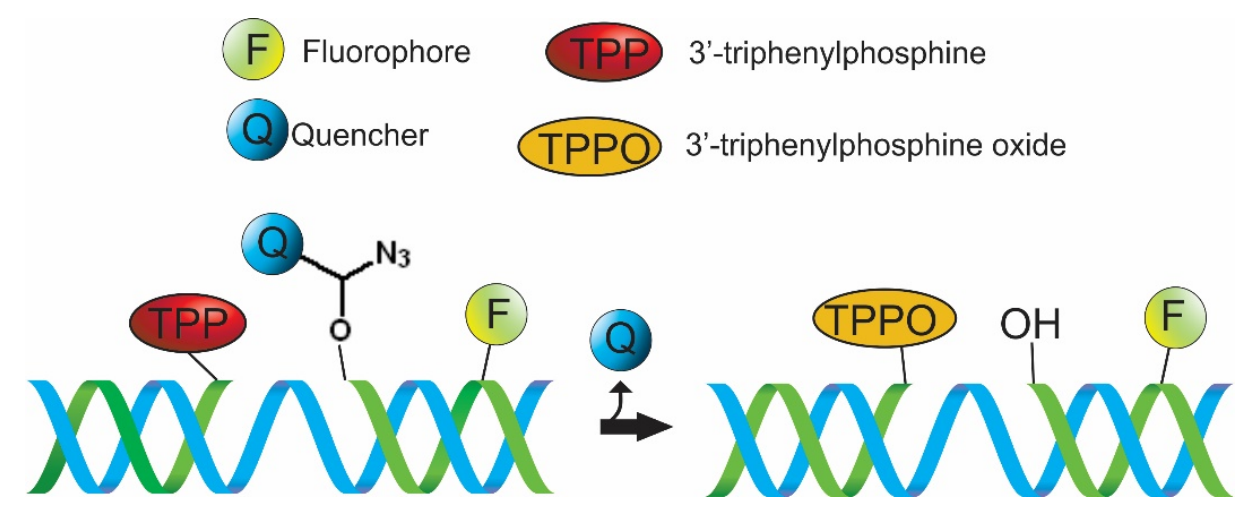

Figure 1.18: Q-STAR OTR system proposed by Farzini and Kool, wherein the Staudinger reaction and subsequent linker cleavage results in the release of a quencher and restoration of fluorescence of the attached fluorophore. ${ }^{79}$

An area of more recent interest in OTR is the incorporation of catalysts into the reaction, such that a molecule released by the triggered reaction would result in the conversion of some freely diffusing reactant in solution. Mokhir, Ogilby, and Gothelf demonstrated that an OTR reaction could trigger the release of a photosensitizer that was previously quenched. ${ }^{80}$ Irradiating with red light resulted in the production of ${ }^{1} \mathrm{O}_{2}$, which resulted in the detectable decomposition of a fluorescent dye. 


\subsection{Aptasensors}

\subsubsection{Aptamer sensor design}

A biosensor is a device comprised of multiple biologically based components, with the most important components being the recognition component and the signal transduction component. ${ }^{81}$ The recognition component is responsible for the binding and recognition of a given target in a complex matrix. The signal transduction component is responsible for transforming the signal of the analyte of interest into some form of detectable signal. ${ }^{82}$ Antibodies were long regarded as the best choice in most biosensor applications, but aptamers have quietly and quickly superceeded them in this role due to the many advantages of aptamers over antibodies. ${ }^{18,34}$ Simplified chemical labeling, known structure switching properties, and minimal batch to batch variation after selection of the aptamer are just a few of the reasons that aptamers are slowly becoming the standard in the biosensor field, and have led to the creation of the new field of aptasensors. ${ }^{18}$ Aptasensors are biosensors in which the recognition and typically the signal transduction event are a direct result of the aptamer-target interaction.

Aptasensor configurations can be subdivided into two categories based on the nature of the target recognition complex. In single-site binding aptasensors, the aptamer will interact with the target at a single site, and it will act as both the recognition and the signal transduction component of the aptamer. ${ }^{18}$ This is most common in small molecule sensors, as it has been shown that small molecules typically associate with their aptamers in binding pockets, where other recognition elements would not have access. Dual site binding sensors, also known as sandwich assays, on the other hand are found in most large 
molecule aptasensors, such as cells and proteins. ${ }^{18}$ In this setup, the analyte is sandwiched between two aptamers, with one acting as the recognition element, and the other acting as the reporter element.

A variety of signal transduction methods have been demonstrated for aptasensors, including electrochemical, optical, and mass-sensitive readouts. Electrochemical sensors are built with an aptamer immobilized on a conductive surface, with some relying on the structural change associated with target binding bringing redox-active labels into the proximity of the surface. ${ }^{83}$ A variety of electrochemical aptasensors have been produced for targets as large as whole cells, viruses, and proteins, to small molecules such as kanamycin, ochratoxin $\mathrm{A}$, and bisphenol $\mathrm{A} .{ }^{84-90}$ A mass-sensitive biosensor is any sensor that relates the target binding to change in the surface upon which the aptamer is immobilized in a mass sensitive fashion (lower signal for lower mass changes), and includes surface plasmon resonance (SPR) and quartz crystal microbalance (QCM) type sensors and are generally considered label free techniques. ${ }^{83}$ Mass sensitive sensors have been developed for a range of protein targets including tubulin, thrombin, and human immunoglobulin E, as well as whole bacterial cells. ${ }^{91-94}$ Optical aptasensors will be discussed in further detail in section 1.4.2.

\subsubsection{Optical aptasensors}

Optical aptasensors are those that convert aptamer-target binding into either a fluorescent signal or a colour change. Fluorescence detection is the most widely used merely due to the ease of chemically labelling aptamers with the wide range of 
fluorophores and quenchers available. ${ }^{34,83}$ Such systems can be built with either single or double reporters, depending on the application. A single reporter system relies on the known structural changes that an aptamer undergoes upon binding to its target to elicit a change in a single appended fluorophore. ${ }^{34}$ The change in structure results in a change in the chemical environment of the attached fluorophore, resulting in a change in the fluorescence properties of the tag such as intensity or anisotropy. Single reporter systems can also be built around the replacement of non-fluorescent nucleotides with fluorescent analogues, such as 3MI (3-methylisoxanthopterin) replacing guanosine. ${ }^{34,95}$ Upon disruption of base stacking, such as in single-stranded regions, these nucleotides will no longer be quenched and thereby result in a fluorescence intensity increase. Fluorescence anisotropy is a result of the change in overall molecular weight and size of the aptamer upon target binding, resulting in a change in its rotational diffusion rate and change in the fluorescence anisotropy of appended fluorophores. ${ }^{34,96}$ Strategies that involve two fluorescent reporters rely on changes in FRET to induce a signal change. This is commonly observed in molecular beacon applications where the formation of a hairpin-loop structure in the aptamer brings the end labeled quencher and fluorophore into proximity to induce quenching. ${ }^{97}$ Target binding disrupts this interaction, increasing fluorescence intensity. ${ }^{34}$

Gold nanoparticles (AuNPs) are commonly used for the development of colourimetric aptasensors due to their ease of use and chemical properties. Due to their unique optical properties of strong localized surface plasmon resonance (LSPR) and high extinction coefficient at the visible wavelength, AuNPs have been used in many colourimetric sensors. ${ }^{83,98}$ One sensor design strategy for AuNPs is networking achieved 
through aptamer crosslinking. For targets with multiple binding epitopes on their surface, AuNP conjugated aptamers can be used to create nanoparticle networks. ${ }^{83}$ These networks result in a colour change from red to purple due to the decrease in inter-nanoparticle distance. This can also be adapted such that an aptamer complimentary to two AuNP conjugated sequences in solution will result in probe binding and colour change, but through the addition of the aptamer target the AuNP network would dissociate, resulting in another colour change. Another strategy relies on the aggregation of AuNPs, which can be controlled using aptamers. AuNPs can be stabilized in solution through association with anions which results in the repulsion of the nanoparticles. ${ }^{83}$ The addition of salt will result in a reduction of static repulsion between AuNPs, resulting in aggregation. By coating the surface of AuNPs with single-stranded DNA such as aptamers, this salt-induced aggregation is limited. ${ }^{98}$ By drawing the aptamer into solution through target binding, they will no longer coat the AuNPs and therefore result in aggregation upon salt addition. ${ }^{83}$

\subsection{Designing a DNA aptamer-based sensor using oligonucleotide templated reactions}

Controlling chemical reactions is of interest in the field of sensor development but is also relevant to the field of spatiotemporal reaction control for its diverse applications. The focus of this work is the fundamental demonstration of controlling chemical reactions using DNA aptamers. There is limited literature precedent for controlling chemical reactions using DNA aptamers, and still less for applying this controlled reaction to the development of sensor technology. This work sought to apply a variety of oligonucleotide 
chemical reactions to aptamer technology for the sensing of aptamer targets in solution. It is hypothesized that an aptamer can be used to both initiate and inhibit an oligonucleotide templated chemical reaction using the target against which the aptamer is selected.

Initial experiments used aptamers as the complementary sequence to two labeled reactive probes as a way of forming a signal-off type sensor. The aptamer sequence would allow the chemical reaction between the two labeled probes by increasing the effective local concentration of the sequences, but upon addition of the target, the amount of unbound aptamer catalyzing this reaction would lower thereby slowing the reaction and resulting in a decrease in signal. These systems were then expanded to include fluorogenic click chemical reactions for greater stability and cleaner reactions. After demonstrating the ability to slow a reaction through the addition of the aptamer target, the ability to initiate a chemical reaction was also demonstrated through two novel systems. The first system sought to demonstrate the ability to take advantage of structure switching aptamers with known structural changes to isolate two reactive elements in the absence of target and bring them into proximity in the presence of target. This was demonstrated using a known Gquadruplex forming thrombin aptamer. Split aptamers were also demonstrated to catalyze chemical reactions and used to develop a sensor for cocaine. 


\section{Chapter: Designing an Ochratoxin A sensor based on Quenched-}

\section{Autoligation probes}

\subsection{Statement of contributions}

All DNA synthesis and testing of the initial QUAL based ochratoxin A sensor was performed with the assistance of undergraduate students Lucas Bonetto, Bouran Hourani, and Evan Monk. Christopher Mattice performed all final experimentation, sensor evaluations, and calculations.

\subsection{Introduction}

\subsubsection{Quenched Autoligation probes}

The nonenzymatic ligation of DNA strands was a field of interest in the early 1990s, with the research groups of Kool and Letsinger being at the forefront. ${ }^{61,63}$ Non-enzymatic ligations of oligonucleotides were initially perceived as a method of synthesizing sequences of greater length than could be produced on the DNA synthesizer, or for the facile incorporation of unnatural bases into these sequences. ${ }^{63}$ It was later demonstrated that the greater utility of these enzymatic ligations was in sensor development, particular for the detection of single nucleotide polymorphisms (SNPs) in the templating sequence, which will be discussed later.

The first demonstration of a templated ligation was in the work of Herrlein and Letsinger, where they demonstrated that two probes labeled with a 3'-phosphorothioate group and a 5'-bromoacetyl group respectively could undergo a spontaneous autoligation 
in the presence of a complementary single-stranded template. ${ }^{61}$ The reactive groups were incorporated into the sequences in such a way that the phosphorothioate and bromoacetyl groups would be nearby upon formation of the DNA duplex with the complementary sequence. ${ }^{61}$ Herrlein et al. demonstrated an alternative method in the following year based on the displacement of a tosyl group by the same phosphorothioate moiety. ${ }^{62}$ The rationale behind this alternative ligation method was an attempt to minimize the distortions introduced through an extended internucleotide bridge caused by the acetyl group. By replacing the bromoacetyl group with a tosyl group, the bridge geometry and charge distribution are much closer to a natural phosphodiester bond with sulfur replacing one of the bridging phosphodiester oxygen atoms. ${ }^{62-63}$

The Kool research group demonstrated that the synthesis of the iodophosphoramidite of thymidine would allow for the incorporation a 5'-iodo group as the electrophilic centre for $\mathrm{S}_{\mathrm{N}} 2$ displacement reactions instead of a tosyl group. ${ }^{64}$ The tosyl functionality required extensive protection schemes and mild synthesis methods to avoid degradation of the tosyl modifier both during and after DNA synthesis, especially during the ammonia deprotection step. ${ }^{63-64}$ Displacement of iodide by the nucleophilic phosphorothioate resulted in rapid ligation of the sequences with minimal background decomposition. It was with this system that the Kool group was first able to demonstrate sequence fidelity in non-enzymatic DNA autoligation reactions. ${ }^{64}$ It was found that the main factor in a successful ligation reaction was the binding affinity of the probes to the template, rather than the geometry of the ligation junction. This allowed for single nucleotide mismatch discrimination in the rate of the reaction. ${ }^{64}$ Single nucleotide 
mismatch detection was also demonstrated by Kool using a phophoroseleonoate in place of a phosphorothioate to increase reaction rates without affecting specificity. ${ }^{65}$ The first demonstration of a point mutation sensitive colourimetric ligation in solution was performed by Kool in 2001, using a FRET pair between a fluorescently tagged 5'iodothymidine probe and unlabeled phosphorothioate complementary to a fluorescently labeled template strand. ${ }^{99}$

The detection of single nucleotide polymorphisms in situ using small oligonucleotide-based probes was of interest in the early 2000s because of its importance in distinguishing strains of bacteria, detecting drug resistance, and evaluating early stages of cancer. ${ }^{100}$ Initially, in situ detection of rRNA in cells relied on FISH (fluorescence in situ hybridization), using fluorophore-labeled targeted probes of 15-30 bases in length. ${ }^{101-}$ 102 This required extensive washing for non-specific binding and imaging posthybridization, with limitations related to low sequence specificity due to the length of the probes and incompatibility with live cells. Quenched oligonucleotide probes were the logical next step, and have the advantage of reducing background signal and eliminating the need for washing steps- making it compatible with live cells. ${ }^{100}$ There are two different categories of quenched probes, being either probes that undergo a conformational change to separate a fluorophore and a quencher (molecular beacons), or probes that are unquenched by a chemical reaction (quenched autoligation probes). A molecular beacon is an oligonucleotide that undergoes a conformational change upon hybridizing to a complementary target, resulting in a fluorescent signal by increasing the distance between the fluorophore and quencher that are attached on either side of its looped hairpin 
structure. $^{97,} 100$ Quenched autoligation probes (QUAL) consist of a probe pair, one containing an internal fluorophore and a 5' electrophilic quencher, and the other probe contains a nucleophilic phosphorothioate at its 3' terminus. ${ }^{100-101}$ By binding adjacently on the target sequence, the nucleophilic group will displace the electrophilic quencher, resulting in a fluorescent signal.

Sando and Kool first proposed quenched autoligation probes in 2002 as an alternative to their original phosphorothioate/iodide DNA ligation reaction. ${ }^{103}$ In this case, a strong fluorescent signal change will be observable by replacing the 5 '-iodothymidine with a 5'-dabsylthymidine labeled quencher sequence containing a fluorescein modifier. The dabsyl modifier includes a sulfonyl group that can be displaced by the phosphorothioate nucleophile to form a dabsylate leaving group. ${ }^{66}$ Separating the dabsyl quencher from the strand containing the fluorophore results in fluorescence restoration. Sando and Kool demonstrated that this system could be used to detect SNPs within hours, but also noted that dabsyl incompletely quenched the fluorescein starting material thereby resulting in an unavoidable background signal. ${ }^{66,103}$ Lack of turnover was also noted in QUAL reactions resulting from increased stability of the probe-template complex after the ligation reaction. ${ }^{66}$ Abe and Kool explored amplification of the signal and increased reaction rate through destabilizing linkers within the probes in $2004 .{ }^{104}$ These destabilizing linkers were incorporated between the dabsyl and the thymidine, and served to destabilize the final linkage between the two oligonucleotides through geometric constraints. In 2008, novel nucleophile chemistry was proposed by Miller, Silverman, and Kool wherein the phosphorothioate molecule was replaced with a phosphorodithioate (PS2) or a 
phosophortrithioate (PS3) using commercial sulfurization reagents. ${ }^{105}$ Although this did serve to increase the rate of the reaction and resulted in a faster readout for the detection of SNPs (rate doubled for PS2 and tripled for PS3), complications related to the stability of the phosophorodithioate and phosphorotrithioate may limit their applications. ${ }^{105}$ In a more recent study, Kleinbaum, Miller, and Kool demonstrated double displacement of dabsyl by nucleophilic phosphorothioate. ${ }^{106}$ A common issue with dabsyl systems is the loss of quencher through latent nucleophiles and autohydrolysis of the quencher in water, resulting in non-specific fluorescence increase. ${ }^{100,105-106}$ It was determined that autohydrolysis of the dabsyl quencher in water resulted in the largest impact on signal to background ratio. In the proposed system, two displacement reactions would have to occur for the duallabeled thymidine residue to produce a substantial fluorescence increase, thereby limiting the effect of the non-specific background reaction. ${ }^{106}$ This resulted in a substantial increase in the signal to background ratio, which was of importance for intracellular measurements.

\subsubsection{Mycotoxins}

\subsubsection{An introduction to mycotoxins and their importance}

Mycotoxins are low molecular weight secondary metabolites produced by filamentous fungi, that serve no understood biochemical role within the fungi. ${ }^{107-108}$ While all mycotoxins are of a fungal origin, it is important to note that not all toxic compounds produced by fungi are mycotoxins- mycotoxins are defined as toxins that are particularly toxic to vertebrates at low concentration. ${ }^{108}$ Other toxins produced by fungi can be 
classified as phytotoxins, which are toxic to plants, and antibiotics, which are toxic to bacteria. ${ }^{108}$ Diseases related to fungi can be classified as either mycoses or mycotoxicoses. Mycoses are a result of the fungi grown on the host, while mycotoxicoses are a result of the toxic fungal metabolites. ${ }^{108}$

Mycotoxins commonly contaminate food commodities, especially cereals such as wheat-maize and barley, and are also found in groundnuts, spices, and dried fruits. Mycotoxicosis is a result of the unintentional consumption of contaminated food products, especially in underdeveloped countries where screening practices and storage practices are less stringent. ${ }^{107-108}$ The two most important drivers of mycotoxin contamination are the need to store crops and the movement of crops outside of their areas of adaptation. Storage of crops, especially in moist conditions, results in the growth of saprophytic fungi. ${ }^{107,109-}$ 110 The toxic effects resulting from mycotoxin consumption can be wide-ranging and include both acute and chronic toxicities.

The most important classes of mycotoxins are those that appear to have the most significant effect on humans. Aflatoxins, of which over 20 derivatives have been isolated, are difuranocoumarin derivates produced primarily by Aspergillus flavus and Aspergillus parasiticus. Aflatoxin $\mathrm{B}_{1}$ is considered the most potent natural carcinogen and is the most commonly produced aflatoxin. ${ }^{107-108}$ Contamination of cereals, nuts, tobacco, figs, and oilseeds are common and result in a family of diseases called aflitoxicoses. Other commonly studied mycotoxins include fumonisins, zearalenone, trichothecenes, and ochratoxins with general structures depicted in Figure 2.1. ${ }^{107-108,110}$ Most mycotoxins are identified through their impact on domesticated livestock, leading to questions in human health and further 
exploration before regulations being recommended by governing bodies. ${ }^{107,111}$
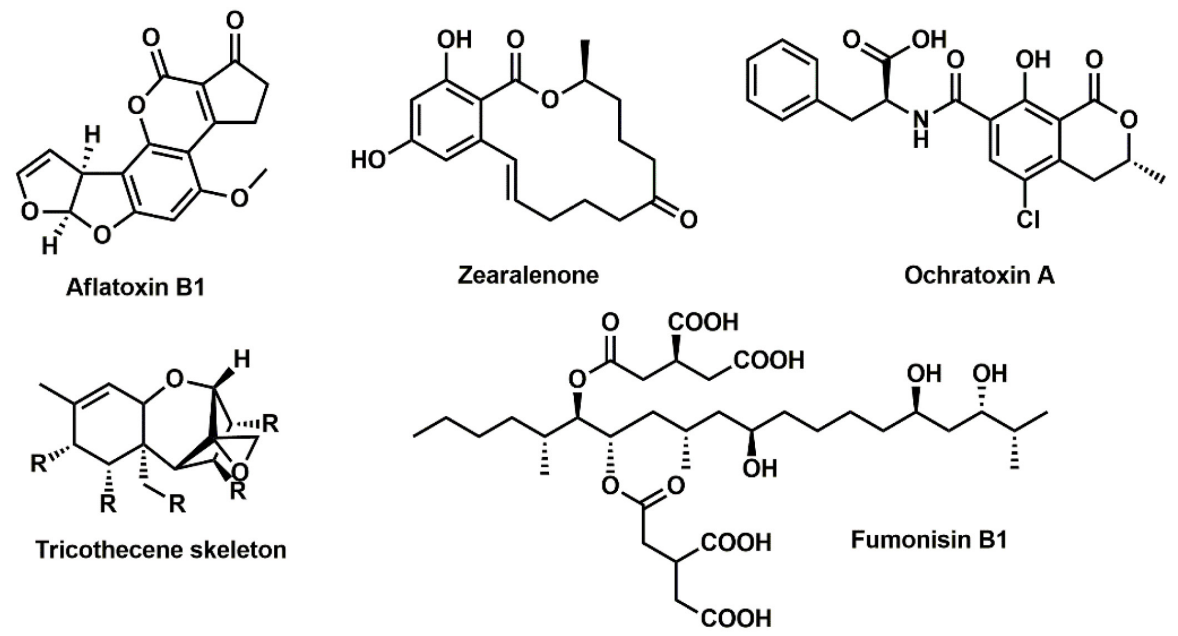

Figure 2.1: Examples and general structures of each of the most common mycotoxin classes.

The economic consequences of mycotoxins are extensive, especially in terms of crop loss. Upon screening, the disposal of contaminated crops is necessary to prevent primary effects on humans through direct consumption, or the secondary effects through consumption of contaminated animal products. ${ }^{110}$ In the United States aflatoxin-related losses alone have accounted for 300 million dollars in loss in food and feed crops per annum, while recent epidemics in Ontario have resulted in $\$ 145$ million in crop loss from deoxynivalenol (DON, a member of the tricothecence class) and head blight from a 1996 outbreak. A similar head blight outbreak in the United States between 1998 and 2000 resulted in over $\$ 870$ million in direct losses. ${ }^{110}$ The detection of one or more mycotoxins above regulated tolerances in a given country triggers a series of tests and assessments and can result in significant crop loss for farmers and labeling of nations as problematic exporters due to contamination. Many crop-based mycotoxins have regulatory tolerances in the $\mu \mathrm{g} / \mathrm{kg}$ range, which results in sampling issues from sample heterogeneity in large 
bulk samples. $^{109}$ A rapid on-site screening method for mycotoxins in harvested crops is essential to eliminate much of the screening issues associated with complicated processes that can result in false positives from sampling error. ${ }^{109}$ Clearing a batch of harvested crops quickly through a simple qualitative test that can be performed by untrained individuals on many samples helps to eliminate false positives and provide better information on genuinely contaminated harvests that require further quantitative analysis. ${ }^{109,} 112$ Such simplified tests could be performed by primary producers, or the processing company, rather than requiring the involvement of the regulator with complicated, time-consuming analysis (i.e. LC-MS/MS). ${ }^{109}$

\subsubsection{Ochratoxin A}

Ochratoxin A (OTA) is a potent nephrotoxic mycotoxin that has a toxic effect in both animal and human populations. ${ }^{107-108,113}$ OTA is a pentaketide derived from dihydrocoumarins coupled to $\beta$-phenylalanine, with the full name L-phenylalanine- $N$-[5chloro-3,4-dihydro-8-hydroxy-3-methyl-1-oxo-1H-2-benzyopyrane-7-yl)carbonyl]-(R)isocoumarin, and is depicted in Figure 2.2. ${ }^{114}$ There are a variety of chemical derivatives of OTA that have been discovered, including ochratoxin B, ochratoxin $\mathrm{C}$, and ochratoxin

$\alpha .^{113}$ The most important characteristic of OTA is its high stability, in that it is highly resistant to acidity and high temperatures, making it quite difficult to remove as a contaminant. $^{113-114}$ Cooking does not remove OTA from contaminated foodstuffs. Thus once it is found in a crop sample above its regulatory limit, it is deemed not fit for 
consumption and must be destroyed. ${ }^{113,115-116}$<smiles>C[C@H]1Cc2c(Cl)cc(C(=O)N[C@@H](Cc3ccccc3)C(=O)O)c(O)c2C(=O)O1</smiles>

Ochratoxin A<smiles>CCOC(=O)[C@H](Cc1ccccc1)NC(=O)c1cc(Cl)c2c(c1O)C(=O)O[C@H](C)C2</smiles>

Ochratoxin C<smiles>C[C@H]1Cc2ccc(C(=O)N[C@@H](Cc3ccccc3)C(=O)O)c(O)c2C(=O)O1</smiles>

Ochratoxin B<smiles>C[C@H]1Cc2c(Cl)cc(C(=O)O)c(O)c2C(=O)O1</smiles>

Ochratoxin alpha

Figure 2.2: The structures of the most common ochratoxins, with ochratoxin A being the most prevalent and the most toxic.

OTA is produced by a range of species of Aspergillus, namely Aspergillus alliaceus, Aspergillus auricomus, Aspergillus carbonarius, Aspergillus glaucus, and Aspergillus niger. ${ }^{113}$ It was initially believed to be produced by a variety of Penicillium species, but it was later determined that the only food-safety relevant species producing OTA in the genus was Penicillium verrucosum. ${ }^{117}$ OTA has been found in a variety of foodstuffs, including barley, oats, rye, wheat, and coffee beans. ${ }^{113,117}$ Cereal products were found to be the highest contributor to OTA in children, while wine has more recently been demonstrated as one of the significant contributors in adults. ${ }^{117}$

Ochratoxin A is linked to a kidney disease found in the Balkans in the 1950s, called Balkan endemic nephropathy $(\mathrm{BEN})$, which is a progressive, untreatable and fatal result of OTA consumption. ${ }^{113-114}$ This led to the understanding of OTA being a potent nephrotoxin, in addition to its liver toxicity, immune suppression, teratogenicity, and more recent 
classification as a potential carcinogen in humans. ${ }^{108,113-114}$ The European Union has established regulatory limits for OTA consumption, with a tolerable weekly intake of 120 ng of OTA per $\mathrm{kg}$ of bodyweight. Limits in foodstuffs such as cereals, cereal products, and coffee have been established to be as low as $3 \mu \mathrm{g} / \mathrm{kg}$ of product. ${ }^{113-115}$ There is currently no regulatory limit in Canada. ${ }^{110}$

The current standardized method for the detection of OTA is based on liquid chromatography coupled to a fluorescence readout. ${ }^{112,115,118}$ Most assays for OTA are based on its known fluorescent nature, simplifying its detection. These analytical methods suffer from being time-consuming, requiring trained personnel, and being quite expensive to operate and maintain. ${ }^{109,112}$ Therefore, alternative strategies for in-field detection would be preferable and aptamers have the potential of fulfilling that role in the future.

\subsubsection{Aptamers for och ratoxin A}

In the literature there are currently 5 published aptamers for ochratoxin A. OTA was the subject of the first aptamer selection for binding to a mycotoxin, as was published by Cruz-Aguado and Penner in 2008. ${ }^{112}$ After selection and sequence optimization they were able to produce a 36 base sequence, called OTA aptamer 1.12.2, that had an experimental $\mathrm{K}_{\mathrm{d}}$ of $100 \mathrm{nM}$ and could be used in assays to detect OTA down to ppb levels. In 2010 Barthelmebs et al. developed two 30 base aptamers against OTA, H8 and H12 with nanomolar binding affinities. ${ }^{119}$ McKeaugue et al. selected two aptamers against OTA in 2014, A08 and B08, which had binding affinities of 290 and $110 \mathrm{nM}$ respectively. ${ }^{120}$ 
Table 2.1: DNA sequences of all known ochratoxin A aptamers.

\begin{tabular}{c|c} 
& DNA sequence \\
\hline $\mathbf{1 . 1 2 . 2}^{\mathbf{1 1 2}}$ & GATCGGGTGTGGGTGGCGTAAAGGGAGCATCGGACA \\
$\mathbf{H 8}^{\mathbf{1 1 9}}$ & GGGAGGACGAAGCGGAACCTGGGTGTGGGGTGATCAAGGGA \\
$\mathbf{H 1 2}^{\mathbf{1 1 9}}$ & GTAGACTCAGAAGACACGCCCGACA \\
$\mathbf{A 0 8}^{\mathbf{1 2 0}}$ & GGGAGGACGAAGCGGAACCGGGTGTGGGTGCCTTGATCCAGGGA \\
$\mathbf{B 0 8}^{\mathbf{1 2 0}}$ & GTCTCAGAAGACACGCCCGACA \\
& AGCCTCGTCTGTTCTCCCGGCAGTGTGGGCGAATCTATGCGTACCGTTCGATA \\
AGCCTCGTCTGTTCTCCCGGGGGATGACAAGCAGACGT \\
GTTGGGGAAGACATTCGGGGGTAAGGTGGTGGTAAC
\end{tabular}

\subsubsection{Proposed sensor design for ochratoxin A}

QUAL applications thus far have been limited to the detection of SNPs either in vivo or in vitro. ${ }^{66-67,100-101,104}$ This project sought to use QUAL probes to demonstrate that oligonucleotide templated reactions could be controlled using an aptamer as the template strand. DNA ligation OTRs rely on the two reactive probes to be brought into proximity through binding to a mutually complementary template strand thereby increasing the effective concentration of the reactive groups and catalyzing a reaction. In the absence of the template strand, these two probes should not be able to interact at the low micromolar concentration that the probes are found in solution, and therefore no ligation reaction would occur. By designing the system such that the template strand is a DNA aptamer, it could be rationalized that binding of the aptamer to its target will result in a conformational change that will result in the aptamer sequence that is complementary to at least one of the probes no longer being accessible for hybridization.

The QUAL based sensor system will be designed using the ochratoxin A 1.12.2 aptamer. A dabsyl probe consisting of a 5'-dabsyl thymidine and containing an internal fluorescein modifier will be used as the electrophilic sequence complementary to the OTA 
aptamer. A thiophosphate sequence will also be synthesized to contain a 3'-thiophosphate and will act as the nucleophilic complementary sequence to the OTA aptamer. Under conditions where ochratoxin A is not present in solution, the aptamer will bind to the two complementary sequences and catalyze the autoligation reaction, resulting in a fluorescent signal increase. If ochratoxin $\mathrm{A}$ is present in solution, a reversible interaction will occur between the aptamer and its target thereby slowing the autoligation reaction. Measurement of fluorescence at a given timepoint would permit qualitative and potentially quantitative information on the presence of ochratoxin A in solution. Though this proposed system does not eliminate the need for a fluorescent readout for the detection of ochratoxin $\mathrm{A}$, it does constitute a step in the direction of controllable chemical reactions using the target of an aptamer and could be expanded to chromogenic applications in the future for an in-field readout as is important in the field of mycotoxin analysis.

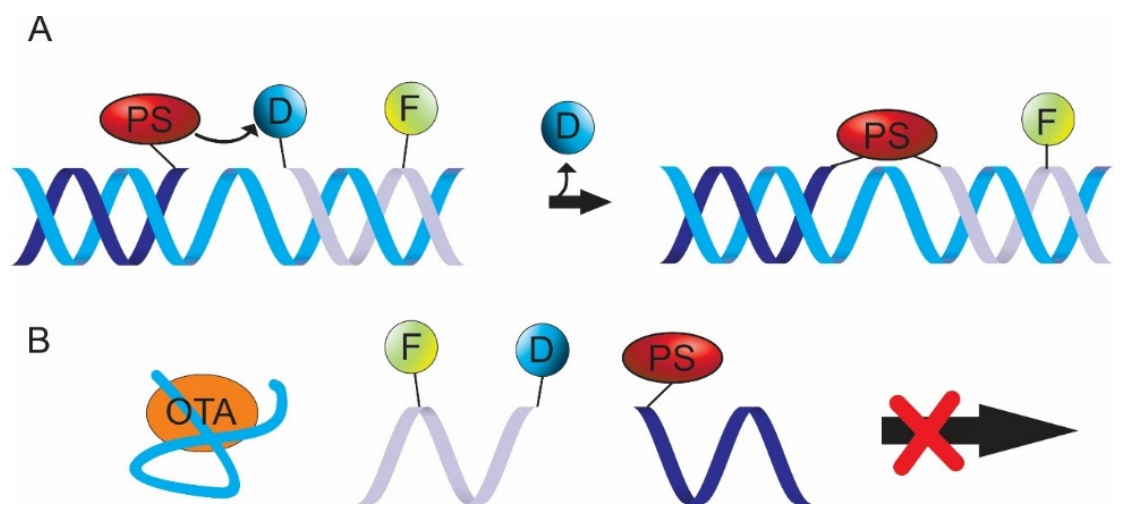

Figure 2.3: A) Catalyzing a quenched autoligation reaction using an ochratoxin DNA aptamer A (light blue), complementary to two probes. One labeled with dabsyl quencher (D) and fluorescein (F), and the other labeled with a 3'-nucleophilic phorphorothioate. B) Addition of ochratoxin A results in aptamer binding, which inhibits the autoligation reaction. This is a simplified representation of the system; dabsyl and fluorescein modifiers are separated by a single base in this and all subsequent figures. 


\subsection{Experimental}

\subsubsection{Materials and equipment}

All oligonucleotide sequences were synthesized using a BioAutomation Mermade 6 oligonucleotide synthesizer using the solid phase phosphoramidite method. 5'-dabsyldT-CE phosphoramidite, fluorescein dT phosphoramidite, sulfurizing reagent II, Pac-dACE phosphoramidite, iPr-Pac-dG-CE phosphoramidite, 5\% phenoxyacetic anhydride in THF Cap Mix A, 0.05M potassium carbonate in methanol, and 3'-phosphate CPG columns were purchased from Glen Research. All necessary reagents for DNA synthesis including standard phosphoramidites, activator, deblock, oxidizer, capping agents, and activator were purchased from Glen Research. Standard controlled pore glass (CPG) columns were purchased from Bioautomation. Biosynthesis grade acetonitrile was purchased from EMD Millipore for use on the DNA synthesizer. Ultra-high purity (UHP) argon was purchased from Praxair for use on the DNA synthesizer. Glen Pak DNA purification cartridges were purchased from Glen Research. All buffers were prepared using EMD Millipore Milli-Q deionized water, and buffer salts were purchased from Sigma Aldrich and BioShop Canada Inc. Buffers were filtered through Corning $0.22 \mu \mathrm{m}$ PES vacuum filtration systems from Sigma Aldrich. Acrylamide/bis-acrylamide 40\% solution, electrophoresis grade ammonium persulfate (APS), $N, N, N^{\prime}, N^{\prime}$-tetramethylethylenediamine (TEMED), ethylenediaminetetracetic acid (EDTA), boric acid, tris(hydroxymethyl) aminomethane (Tris), and urea were purchased from Bioshop Canada Inc. Ochratoxin A, $1 \mathrm{mg} / \mathrm{mL}$ in DMSO from Petromyces albertensis was purchased from Sigma Aldrich. PAGE gels were imaged using an Alpha Innotech multi-image light cabinet. UV/Visible absorption spectra 
were acquired using a Varian Cary 300 Bio/UV visible spectrophotometer. Fluorescence spectra were acquired using a Horiba FluoroLog-3.

\subsubsection{Buffer}

Table 2.2: Buffer composition for ochratoxin A quenched autoligation probe sensor experiments.

\begin{tabular}{c|c|c} 
Buffer name & Composition & $\mathrm{pH}$ \\
\hline & $10 \mathrm{mM} \mathrm{Tris-borate}$ & \\
& $0.1 \mathrm{mM} \mathrm{DTT}$ & 7.4 \\
QUAL buffer & $60 \mathrm{mM} \mathrm{NaCl}$ & \\
& $5 \mathrm{mM} \mathrm{KCl}$ & \\
& $5 \mathrm{mM} \mathrm{MgCl}_{2}$ &
\end{tabular}

\subsubsection{DNA synthesis, purification, and quantification}

Table 2.3: Oligonucleotide sequences used in the QUAL experiment for designing an ochratoxin A sensor. TD is the dabsyl-thymidine modifier, TF is the fluorescein-thymidine modifier, PS is used to indicate the location of the thiophosphate modifier, and PO is a 3'-phosphate modification.

\begin{tabular}{|c|c|}
\hline Oligonucleotide & Sequence \\
\hline $\begin{array}{c}\text { OTA aptamer 1.12.2 } \\
\text { Dabsyl Probe } \\
\text { Thiophosphate probe } \\
\text { Phosphate probe } \\
\text { Internal thiophosphate }\end{array}$ & 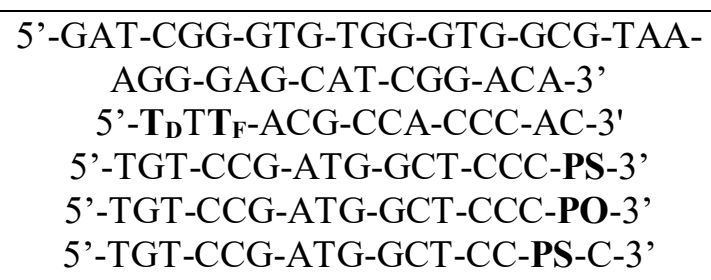 \\
\hline
\end{tabular}

The ochratoxin A 1.12.2 aptamer was synthesized under the standard conditions recommended by Bioautomation, using standard phosphoramidites. $1 \mu$ mol 5'-DMT-dA $1000 \AA$ columns were used for the synthesis. The thiophosphate probe was synthesized starting from 5'-phosphate CPG $1000 \AA$ columns and indicating to the machine that a universal column was being used instead of one already containing a base. For the first 
base added, the oxidizing step was replaced with a sulfurizing step, in which the instrument injected sulfurizing reagent II in place of oxidizing solution. The remainder of the sequence was synthesized under standard conditions with standard phosphoramidites. The phosphate probe was synthesized in the same way as the thiophosphate probe, but the normal oxidizing step was performed instead of the sulfurizing step. Internal thiophosphate was synthesized using a $1 \mu$ mol 5'-DMT-dC $1000 \AA$, with a sulfurizing step after the first base. Both the ochratoxin A 1.12.2 aptamer and the thiophosphate probe were synthesized such that the final deblocking step was omitted, leaving a 5'-DMT group on the final base to allow for Glen Pak purification. For the dabsyl probe, two different modifiers were injected into the machine at the appropriate times, one being 5'-dabsyl dT CE phosphoramidite dissolved to $0.067 \mathrm{M}$ in acetonitrile, and the other being fluorescein $\mathrm{dT}$ phosphoramidite dissolved to $0.067 \mathrm{M}$ in acetonitrile. For the dabsyl probe, ultramild DNA synthesis conditions were followed, requiring the use of Pac-dA-CE phosphoramidite for all adenine bases, and ipr-Pac-dG-CE for all guanine bases, and ultramild Cap Mix A (5\% phenoxyacetic anhydride in THF) in place of the standard Cap Mix A. Coupling times for the two modifiers were also extended to 10 minutes from the standard 1-minute coupling time, and the final deprotection step was omitted.

Upon synthesis completion, the thiophosphate probe, phosphate probe, internal thiophosphate probe, and the OTA aptamer 1.12.2 synthesis columns were removed from the synthesizer, and the contained CPG support beads were transferred into a microcentrifuge tube. The CPG beads were deprotected in $1 \mathrm{~mL}$ of $28 \% \mathrm{NH}_{4} \mathrm{OH}$ for 16 hours at $55^{\circ} \mathrm{C}$, before proceeding to Glen Pak purification. The CPG beads for the dabsyl 
probe were also transferred into microcentrifuge tubes, but in this case, were deprotected for 4 hours at room temperature in $0.05 \mathrm{M} \mathrm{K}_{2} \mathrm{CO}_{3}$ in methanol before proceeding to Glen Pak desalting.

\subsubsection{Glen Pak DNA purification and desalting}

The OTA aptamer 1.12.2, thiophosphate probe, phosphate probe, and internal thiophosphate probe sequences were purified on the Glen Pak cartridge. The CPG deprotection solution in $28 \% \mathrm{NH}_{4} \mathrm{OH}$ was decanted into a new microcentrifuge tube and the beads were washed with $100 \mathrm{mg} / \mathrm{mL} \mathrm{NaCl}$ in deionized water, before decanting and mixing with the initial deprotection solution. One Glen Pak cartridge for each of the six synthesis columns was loaded onto a Phenomenex 12 port vacuum manifold with an attached oil-free vacuum pump and rinsed with $0.5 \mathrm{~mL}$ of acetonitrile followed by $1 \mathrm{~mL}$ of 2.0 M triethyammonium acetate (TEAA). The ammonium hydroxide/ $\mathrm{NaCl}$ deprotection solution was loaded onto each column in turn $1 \mathrm{~mL}$ at a time, and then rinsed twice with 1 $\mathrm{mL}$ of $5 \%$ acetonitrile in $100 \mathrm{mg} / \mathrm{mL}$ sodium chloride. The columns were then deprotected with two $1 \mathrm{~mL}$ aliquots of freshly prepared $2 \%$ trifluoroacetic acid in deionized water, at which point a bright orange band was observed. Rinsing each column twice with $1 \mathrm{~mL}$ of deionized water removed the orange bad. The collection tubes under the vacuum manifold were then switched, and the target sequence eluted using a $1 \mathrm{~mL}$ solution of $50 \%$ acetonitrile in deionized water with $0.5 \%$ ammonium hydroxide. The eluted samples were then dried on a Savant AES2010 SpeedVac cryopump overnight before resuspension in deionized water and quantification. 
For the dabsyl probe, a Glen-Pak desalting protocol was followed, wherein the potassium carbonate in methanol deprotection solution was diluted to $20 \mathrm{~mL}$ in $0.1 \mathrm{M}$ TEAA. The cartridges were prepared as before, and the diluted deprotection solution was added to each of the columns. After loading, each of the columns was rinsed twice with 1 $\mathrm{mL}$ of deionized water. The collection tubes were switched for each of the columns, and the dabsyl probe eluted in $1 \mathrm{~mL}$ of $50 \%$ acetonitrile in deionized water. The eluted samples were then dried on a Savant AES2010 SpeedVac cryopump overnight before resuspension in deionized water and preparation for polyacrylamide gel electrophoresis.

\subsubsection{Polyacrylamide gel electrophoresis}

The dabsyl probe was purified using a $19 \%$ denaturing polyacrylamide gel. The gels were prepared by mixing $63 \mathrm{~g}$ of urea with $75 \mathrm{~mL}$ of acrylamide solution (acrylamide/bisacrylamide $40 \%$ solution), and $30 \mathrm{~mL}$ of $5 \mathrm{x}$ TBE buffer (45 mM Tris, 45 $\mathrm{mM}$ boric acid, $1 \mathrm{mM}$ EDTA). The solution was stirred while heating to $37^{\circ} \mathrm{C}$ and filtered while hot through Whatman No. 1 filter paper. Once cooled to room temperature, $900 \mu \mathrm{L}$ of $10 \%$ ammonium persulfate and $70 \mu \mathrm{L}$ of TEMED were added to the stirred solution. The gel solution was then poured into the casting apparatus for the Hoefer SE600 Chroma standard dual-cooled gel electrophoresis assembly. The gels were pre-run for 20 minutes using a constant voltage of $300 \mathrm{~V}$. During the pre-running, the dried dabsyl probe samples were resuspended in $300 \mu \mathrm{L}$ of deionized water, and then $300 \mu \mathrm{L}$ of formamide was added to each sample. The samples were heated to $55^{\circ} \mathrm{C}$ for 5 minutes before loading into the gel, with each column of synthesized DNA having its own gel. The gels were run for 1.5 hours, before 
imaging on a $F_{254}$ TLC plate in the Alpha Imager gel cabinet set on the epi-UV $(\lambda=254 \mathrm{~nm})$ and fluorescence settings $\left(\lambda_{\mathrm{ex}}=302 \mathrm{~nm}\right)$. The relevant band for the dabsyl probe was manually excised from the gel and placed in a $50 \mathrm{~mL}$ Falcon tube. The probe was eluted from the gel using a crush and soak method using deionized water and incubated on a New Brunswick Scientific Innova 40 incubator/shaker at $37^{\circ} \mathrm{C}$ for 48 hours.

After extraction, the solution was filtered through $0.22 \mu \mathrm{m}$, low retention, cellulose acetate syringe filters. The samples were then made up to $20 \mathrm{~mL}$ in $0.1 \mathrm{M}$ TEAA and desalted according to the previously described Glen Pak desalting protocol.

\subsubsection{DNA quantification}

All three probes were quantified by measuring their optical absorbance at $260 \mathrm{~nm}$ when dissolved in deionized water. By considering the molar extinction coefficients as determined through nearest neighbour analysis and the molar absorptivity of each of the modifiers used, an extinction coefficient can be determined. Using the Beer-Lambert law, the solution concentration of the DNA can be back-calculated. Post quantification, all DNA sequences were submitted for electrospray mass spectrometry for molecular weight confirmation by Novatia LLC. Mass spectrometry was performed using a reverse phase HPLC with direct spray into an LTQ-Orbitrap with deisotoping and deconvolution of the results undertaken using Promass software.

\subsubsection{Proof of QUAL reaction study and optimization}

Quenched autoligation reactions using a DNA aptamer were evaluated by gel 
electrophoresis to determine the extent of probe ligation. In the first set of reactions, the dabsyl probe, ochratoxin A 1.12.2 aptamer, and thiophosphate probe were combined at 10 micromolar concentration each in the QUAL reaction buffer at a $100 \mu \mathrm{L}$ total reaction volume. The reactions were run for 1 hour at room temperature with mixing every 10 minutes. Reactions were also performed in the absence of each of the probes in turn to determine the effect of background reactions. After the 1-hour reaction time, all the samples were diluted with $100 \mu \mathrm{L}$ of formamide and loaded into separate lanes of a $19 \%$ PAGE. Gels were run at $300 \mathrm{~V}$ constant for 1.5 hours, and then imaged under UV-light in the Alpha Imager to determine the extent of the reaction. The same reaction set was performed with the substitution of the phosphate probe for the thiophosphate probe, and again for the internal thiophosphate probe in place of the thiophosphate probe. Both reaction sets were evaluated on a 19\% PAGE and imaged under UV-light in the Alpha Imager.

\subsubsection{QUAL experiments in the presence of ochratoxin A}

\subsubsection{General protocol for QUAL experiment with ochratoxin A}

All tests in the presence of ochratoxin A were evaluated using a 10 micromolar concentration of each of the DNA sequences at a total reaction volume of $100 \mu \mathrm{L}$ in triplicate. Ochratoxin A was diluted in DMSO so that the final reaction concentrations in the reaction tube ranged from $1 \mathrm{mM}$ to $1 \mathrm{pM}$ ochratoxin A. Reactions were prepared so that the reaction in the presence of a given concentration of ochratoxin A (DTO+Tox) could 
be compared to the reaction in the absence of ochratoxin A (DTO). Reactions with the omission of each of the probes, in turn, were also evaluated to determine background reactions. Reactions were set up by adding toxin to the QUAL buffer along with the OTA aptamer. Toxin volumes were calculated so that DMSO comprised $1 \%$ of the total reaction

volume, being $1 \mu \mathrm{L}$, and any reactions that did not contain toxin were supplemented with $1 \mu \mathrm{L}$ of DMSO. After mixing of the OTA aptamer, toxin and buffer, the solution was heated to $55^{\circ} \mathrm{C}$ and then cooled to room temperature. The dabsyl probe was then added to the mixed reactions, and the reaction initiated, and timer started with the addition of the thiophosphate probe. The reactions were run for 1 hour at room temperature with mixing every 10 minutes, and stopped through the addition of $100 \mu \mathrm{L}$ of formamide and heating to $55^{\circ} \mathrm{C}$.

Upon reaction completion, the sample was divided in half, with one half loaded into separate lanes of a $19 \%$ gel. The gel was run for 1 hour at a constant $300 \mathrm{~V}$ and then imaged under both UV and fluorescence modes in the Alpha Imager. The other half of the reaction was analyzed by fluorescence spectroscopy on a Fluorolog3. From each reaction, $70 \mu \mathrm{L}$ was loaded into a quartz fluorescence cuvette, and the emission spectrum measured between 500 and $600 \mathrm{~nm}$ with an excitation wavelength of $494 \mathrm{~nm}$ and $3 \mathrm{~nm}$ slit widths.

\subsection{Results and discussion}

\subsubsection{Incorporation of thiophosphate and dabsyl/fluorescein modifiers on DNA} sequences

The ochratoxin A aptamer was synthesized under standard DNA synthesis 
conditions on the Bioautomation MerMade6 synthesizer. Yields as high as $2.1 \mu \mathrm{mol}$ of DNA were observed from six synthesis columns after purification.

Finding the correct synthesis conditions on the DNA synthesizer for incorporation of the thiophosphate modifier at the 3'-terminus of the thiophosphate probe proved difficult. By purchasing universal 3'-phosphate CPG beads, a 3'-phosphate could be installed onto the DNA rather than an internal phosphate or phosphorothioate bridge. These 3'-phosphate CPG beads are simply a linker with a hydroxyl group attached, which will attack the subsequently added phosphoramidite. The subsequent sulfurization or oxidation step will result in the formation of a 3'-thiophosphate or 3'-phosphate respectively, with the phosphorous being provided by the first phosphoramidite added. Using this method, both a thiophosphate probe and a phosphate probe were synthesized. All three probes were synthesized in the $800-1100 \mathrm{nmol}$ range off six synthesis columns, with the high yield being a result of the short sequence and minimal internal modification.

Synthesis of the dabsyl probe required the use of two modifier positions on the DNA synthesizer to incorporate both dabsyl and fluorescein modification. Due to the lability of dabsyl, ultramild synthesis conditions were recommended by the manufacturer for the dabsyl-dT phosphoramidite. This involves the use of a phenoxyacetic anhydride based capping agent as well as DNA phosphoramidites with weaker protecting groups. This allows for rapid deprotection under mild conditions in room temperature potassium carbonate in methanol. The yield of the dabsyl probe could be as high as $1.2 \mu$ mol off 6 synthesis columns.

The synthesis of the double modified dabsyl probe, the thiophosphate probe, and 
the ochratoxin A aptamer 1.12.2 were confirmed through electrospray ionization mass spectrometry (ESI-MS) molecular weight confirmation analysis. The synthesized double modified dabsyl probe was found to have its expected molecular weight of $4943.8 \mathrm{~g} / \mathrm{mol}$ (appendix Figure A.3), while the thiophosphate was confirmed at a molecular weight of $4286.9 \mathrm{~g} / \mathrm{mol}$ (appendix figure A.2), and the ochratoxin A aptamer at $11311.9 \mathrm{~g} / \mathrm{mol}$ (appendix figure A.1).

\subsubsection{Demonstration of OTR functionality in the absence of ochratoxin A}

Before assessing the ability of the QUAL system coupled with ochratoxin A aptamer 1.12.2 to act as a sensor, the ability of the aptamer to catalyze a QUAL reaction had to be demonstrated. To assess the system, three different analogues of the complementary thiophosphate probe were synthesized, being the traditional 3 '-terminus thiophosphate, the 3'-terminus phosphate, and an internal phosphorothioate linkage in between the final bases at the 3'-terminus. The first assessment of the system for catalyzing a QUAL type reaction used the ochratoxin A 1.12.2 aptamer and the complementary thiophosphate probe along with a dabsyl modified probe. Unlike the dabsyl probe used in QUAL reactions, this probe only contained the 5'-dabsyl dT modifier with no fluorescein dT modifier. This was synthesized to minimize costs in the proof of concept system and was not expected to have any effect on the ligation reaction. As shown in Figure 2.4, the expected reaction is the nucleophilic attack of the phosphorothioate on the dabsyl modifier with $\mathrm{S}_{\mathrm{N}} 2$ displacement of the quencher resulting in ligation of the two strands complementary to the ochratoxin A aptamer. When the reaction was performed, a 
denaturing PAGE gel was run, and the reaction of all three sequences run together in triplicate. As shown in Figure 2.4, when all three sequences are mixed (middle 3 lanes, DTO), a new heavy product band is observed on the gel, indicative of the successful ligation. When the two complementary strands undergo a ligation reaction, a new heavier band is expected to form, but it should not be of greater mass than the ochratoxin A aptamer as the ligation of a 14 and 15 base sequence will not be of greater mass than a 36-base sequence. In the observed gel, the new product band appears above the ochratoxin A band, indicating that it is likely of higher mass, and therefore mass spectrometric confirmation was performed to identify the new product band and confirm the ligation reaction. Based on the ESI-MS results, the excised top band consisted of two DNA sequences, equivalent to the expected ligation product and the ochratoxin A 1.12.2 aptamer sequence (appendix figure A.6). No ligated product is observed in the absence of ochratoxin A aptamer, indicating that the aptamer must be present for the ligation to occur at this $10 \mu \mathrm{M}$ reaction concentration. Based on these results, a complementary ochratoxin A 1.12.2 aptamer can facilitate the strand ligation in a QUAL type reaction in the absence of the fluorescein reporter tag. 

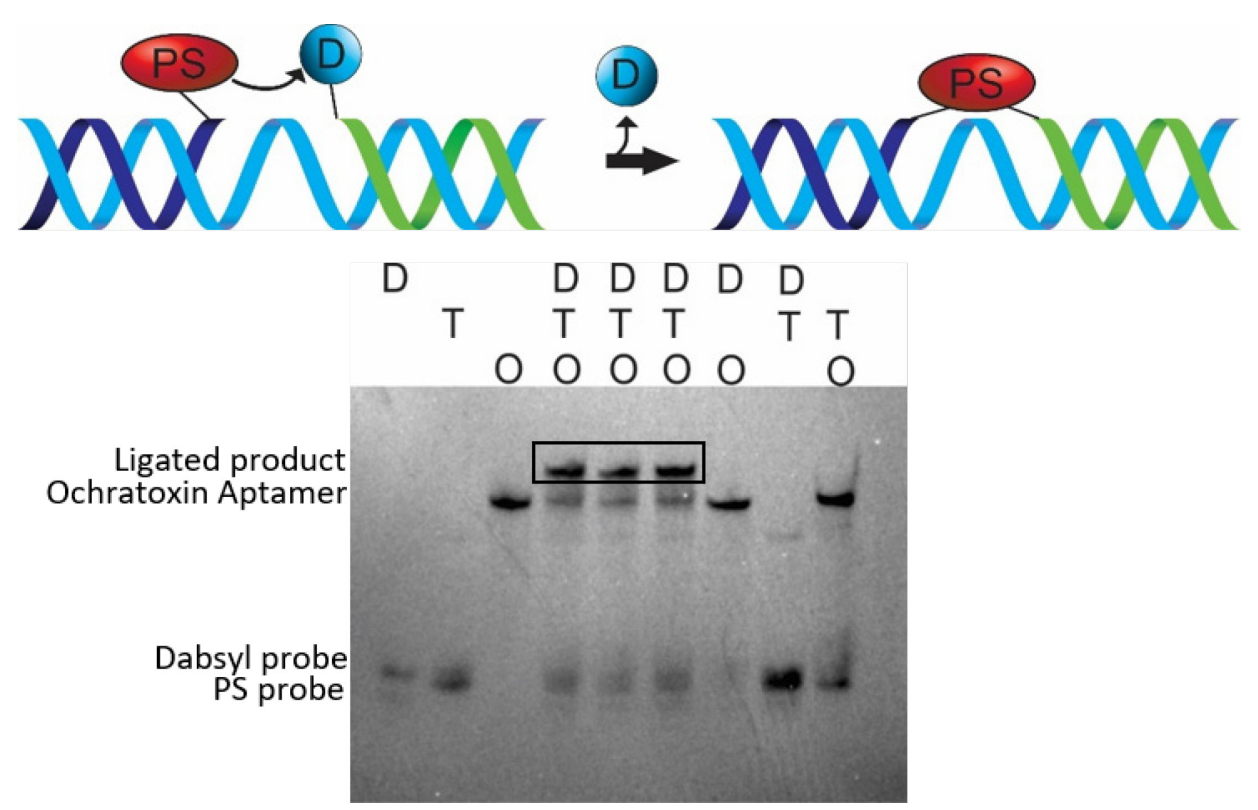

Figure 2.4: Representation of the ligation reaction between a dabsyl-quencher labeled probe (D) and a phosphorothioate labeled probe (T, labeled with PS), complementary to the ochratoxin A aptamer (light blue, 0 ). The circled bands in the DTO lanes indicate the ligated species. The identity of the bands in the gel is indicated by the labels on the left indicating their location in the gel.

Replacement of the thiophosphate sequence with a 3'-phosphate labeled sequence allowed for the testing of the importance of the nucleophilic sulfur atom in the thiophosphate. It is expected that no reaction will occur in the absence of the nucleophile, as had been surmised previously by the Kool group. ${ }^{66,105}$ Upon running the reaction as shown in Figure 2.5, no product band was observed as had been seen in the presence of the appropriate nucleophilic thiophosphate probe. In the lanes containing all three of the sequences (DPO), the three bands for the starting material are observed with no additional sequence, just as in the lane run in the absence of the ochratoxin A aptamer (DP). It can, therefore, be concluded from this simple test that the phosphate does not possess sufficient 
nucleophilic character to displace the dabsyl leaving group, resulting in no QUAL reaction as expected.

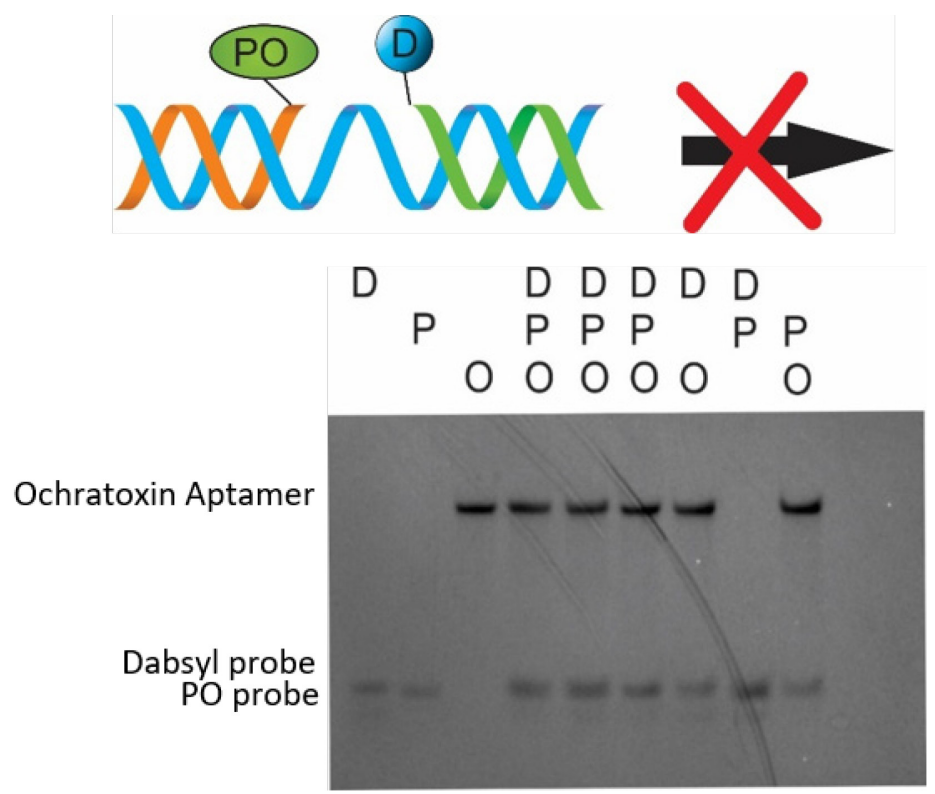

Figure 2.5: Representation of the disabled reaction between a dabsyl-quencher labeled probe (D) and a phosphate labeled probe (P, labeled with PO), complementary to the ochratoxin A aptamer (light blue, $\mathbf{O})$. In the DPO lane no new ligated product is observed. The identity of the bands in the gel is indicated by the labels on the left indicating their location in the gel.

In the final QUAL reaction assessment in the absence of toxin, the phosphorothioate group was placed within the sequence instead of at the 3'-terminus as would be expected for a QUAL reaction. With the phosphorothioate nucleophile being restricted to the middle of the chain, the likelihood of the correct geometry for nucleophilic attack on the dabsyl modifier is unlikely. As shown in Figure 2.6, reaction of the internal thiophosphate with the dabsyl probe in the presence of the complementary ochratoxin A aptamer resulted in no ligated product as expected. It is therefore important that the nucleophilic thiophosphate modifier be located at the 3'-terminus, otherwise strand ligation will not occur as was seen. 


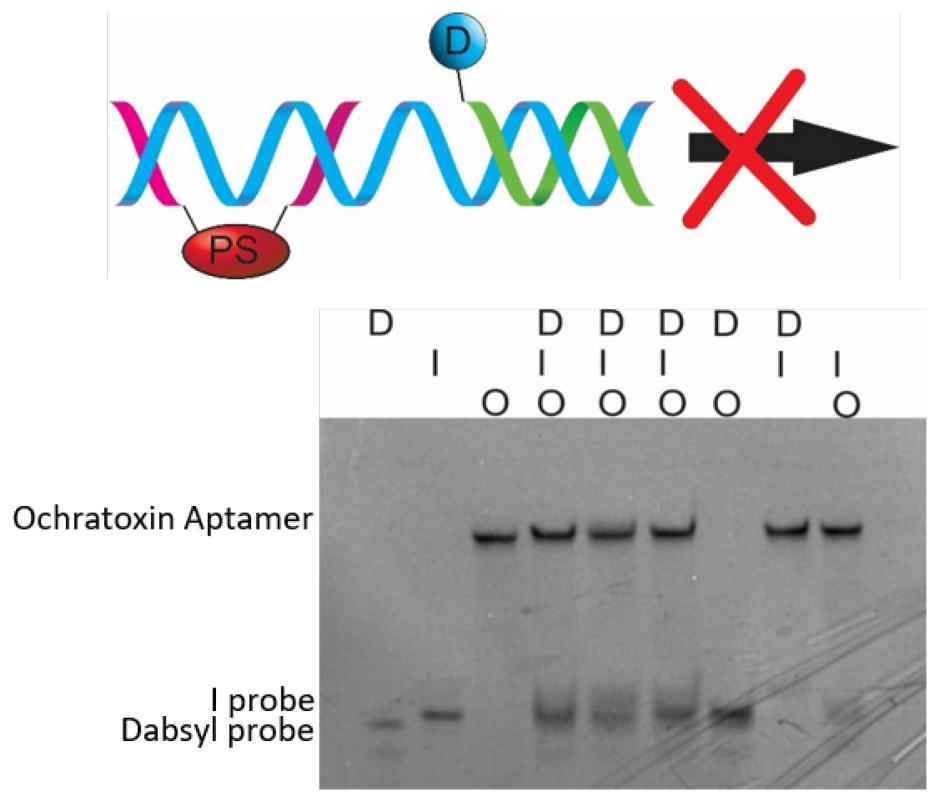

Figure 2.6: Representation of the disabled ligation reaction between a dabsyl-quencher labeled probe (D) and an internal phosphorothioate labeled probe (I, labeled with internal PS), complementary to the ochratoxin A aptamer (light blue, $O$ ). In the DIO lane, no ligation product is observed. The identity of the bands in the gel is indicated by the labels on the left indicating their location in the gel.

A new dabsyl oligo was then synthesized, this time containing both the 5'-dabsyl$\mathrm{dT}$ modifier and the fluorescein-dT modifier two bases in from the 5'-terminus as is typically designed in a QUAL sensor. The reaction of the new dabsyl oligo with the 3'terminus thiophosphate and the ochratoxin A aptamer was assessed under the same conditions as the three previous reactions, and evaluated by gel as shown in Figure 2.7. It is expected that this ligation would readily occur as had been seen in the dabsyl probe without fluorescein and would generate a fluorescent band through loss of the dabsyl quencher in the $\mathrm{S}_{\mathrm{N}} 2$ displacement. As expected, the ligation reaction resulted in a new band forming and upon fluorescence imaging of the gel it was seen that this newly formed 
band was fluorescent, as seen in the gels in Figure 2.7. As seen in Figure 2.7, incomplete separation of the ochratoxin A aptamer and the newly ligated sequence results in streaking of the fluorescent product.

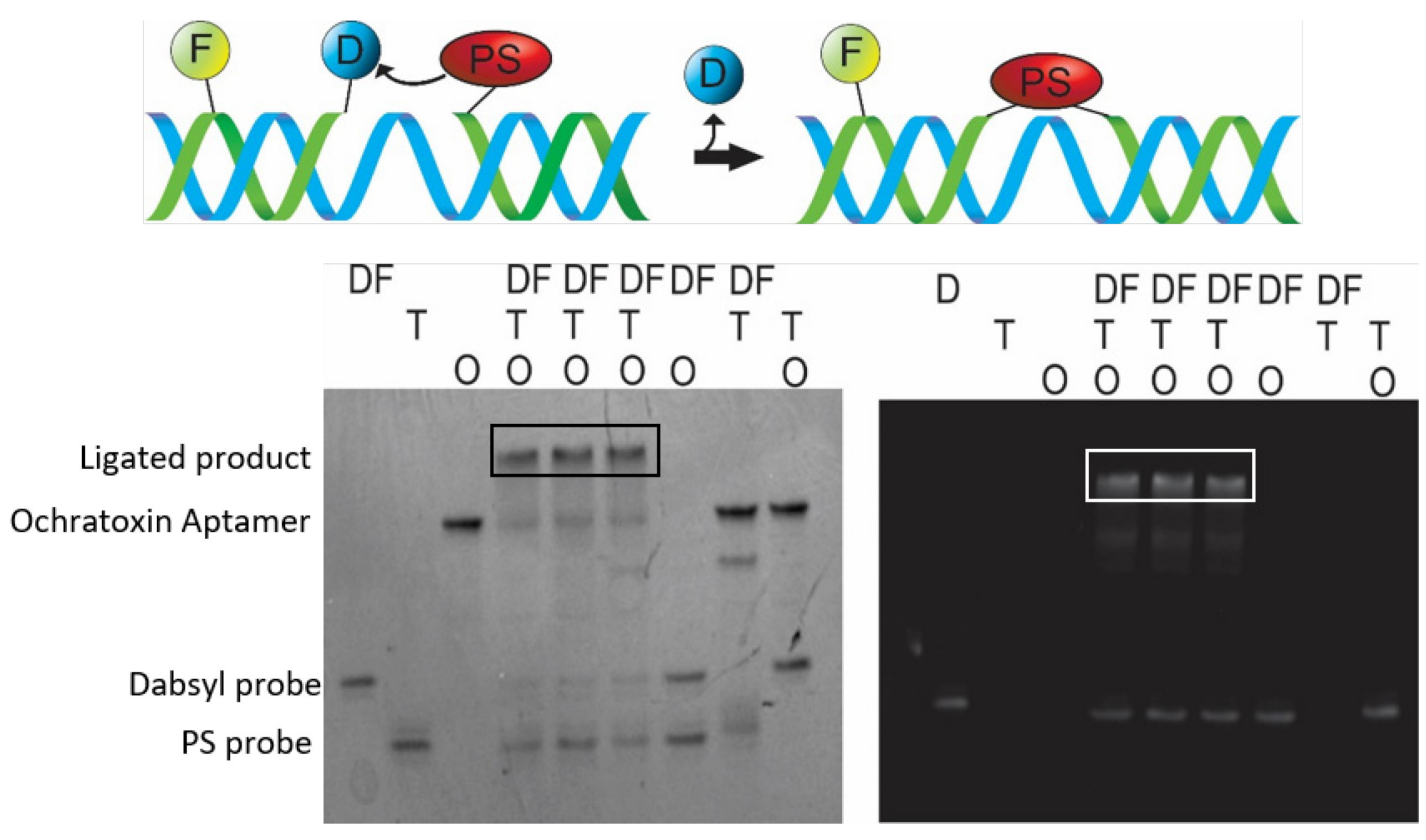

Figure 2.7: Representation of the ligation reaction between a dabsyl-quencher and fluorescein labeled probe (DF) and a phosphorothioate labeled probe (T, labeled with PS), complementary to the ochratoxin A aptamer (light blue, $O$ ). The left gel is imaged under UV-light, while the right gel is imaged using a fluorescent lamp. In the presence of the dual modified dabsyl oligo, a new ligated product is observed as indicated by the square outline. This newly ligated product is highly fluorescent as would be expected from quencher loss. The identity of the bands in the gel is indicated by the labels on the left indicating their location in the gel.

Having established the reaction conditions for the QUAL reaction in the presence of ochratoxin A aptamer and undergone a series of tests reaction with thiophosphate analogues, the next step was testing the turn-off of the QUAL reaction using the ochratoxin A target. 


\subsubsection{Demonstration of the effect of high levels of ochratoxin A on the measured}

\section{fluorescence}

As a proof of concept, it was decided that testing the system with a high concentration of ochratoxin A, well above regulatory limits and expected levels in any realworld sample, would establish a starting point for determining the relevance of this system. A $10 \mu \mathrm{M}$ reaction was run over 2 hours using the double modified dabsyl probe in the presence of the 3'-thiophosphate sequence and the ochratoxin A 1.12.2 aptamer, with and without $1 \mathrm{mM}$ ochratoxin A. The fluorescence spectrophotometric results of the experiment are shown in Figure 2.8 and Figure 2.9. It is expected that in the absence of ochratoxin A, the reaction will occur readily and result in a high fluorescence output as had been observed in the previous gels. In the absence of the aptamer, or in the absence of both the aptamer and the thiophosphate no reaction is expected and therefore a measurement of the intrinsic background fluorescence of the dabsyl probe will be observed. Upon addition of toxin, the aptamer will undergo a reversible interaction with its target, resulting in limited binding of the two complementary sequences and thereby slow the reaction between the dabsyl and the thiophosphate probes in solution. Under ideal circumstances, the addition of toxin would completely inhibit the reaction and therefore result in a fluorescence output equal to the background of the dabsyl probe. Due to the reversibility of the aptamer-target interaction, and the favourable interaction with the two complementary probes, the background reaction in the presence of toxin cannot be eliminated. This parallels the trend observed in Figure 2.8, wherein the fluorescence output 
at $520 \mathrm{~nm}$ from the fluorescein fluorophore for the DTO reaction in which all three probes are mixed is significantly greater than when compared to the DTO+toxin reaction in which $1 \mathrm{mM}$ ochratoxin A was added. Both fluorescence readouts are still higher than the background observed for $\mathrm{D}$ alone or $\mathrm{D}$ and $\mathrm{T}$ mixed together.

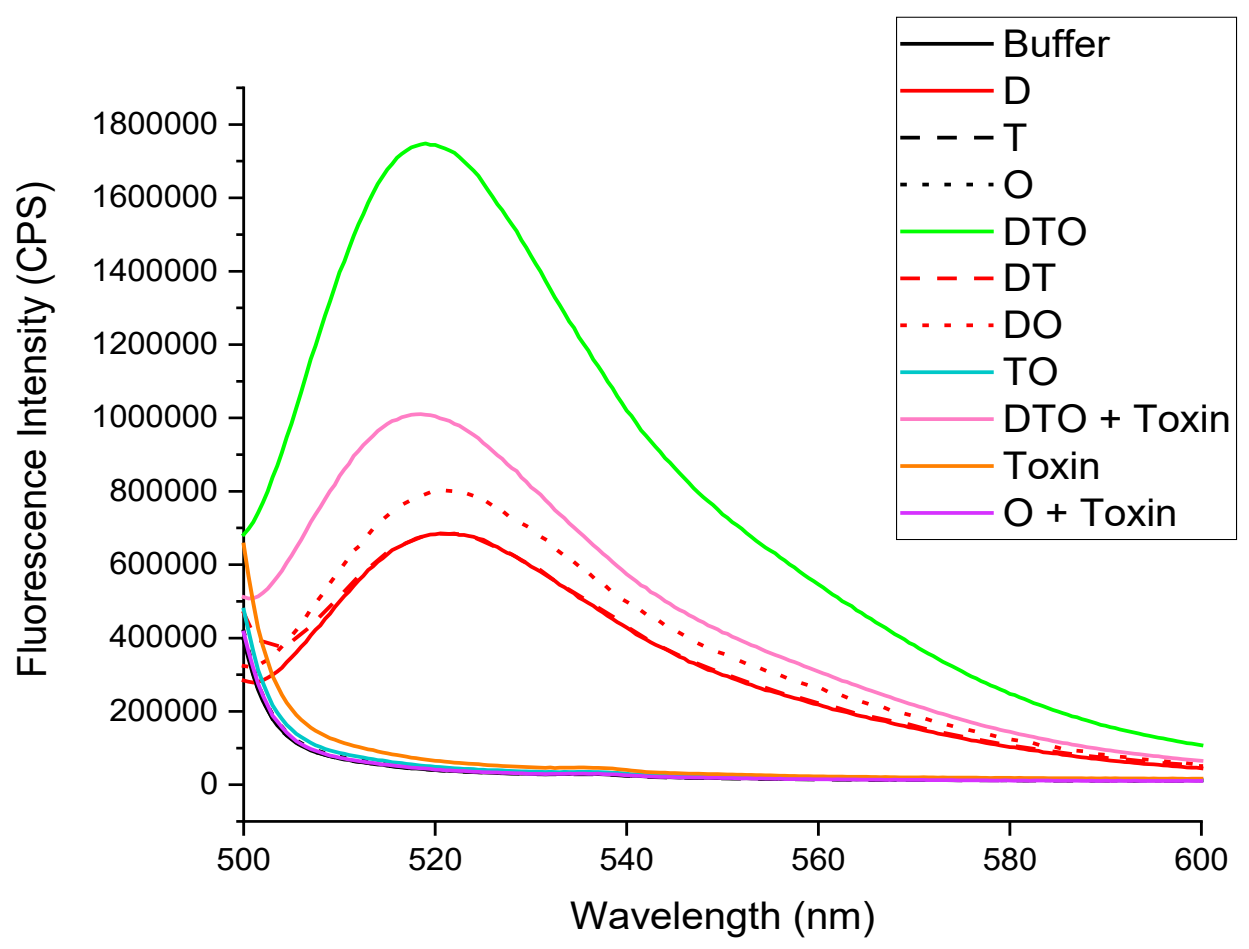

Figure 2.8: Fluorescence spectrum of the ligation reaction between the dabsyl-fluorescein labeled probe (D), thiophosphate probe (T), and the complementary OTA aptamer $(O)$, in the presence and absence of $1 \mathrm{mM}$ OTA concentration. $\lambda_{\mathrm{ex}}=494 \mathrm{~nm}, \lambda_{\mathrm{em}}=500-600 \mathrm{~nm}$. In the presence of all three sequences (DTO) the fluorescence intensity of the reaction is much greater than when ochratoxin A at $1 \mathrm{mM}$ concentration is added to the reaction (DTO + toxin).

The results were distilled into a simplified bar graph as shown in Figure 2.9 to denote the trend in fluorescence intensity in the presence and absence of ochratoxin A. It is important to note that the toxin has a low fluorescence output at this excitation and emission wavelength, in contrast to the known fluorescence output of ochratoxin A with 
an excitation wavelength of $330 \mathrm{~nm}$ and emission of $412 \mathrm{~nm}$. The difference in the fluorescence emission between the reaction in the presence and absence of toxin can also clearly be seen in this figure. Based on these results, it was decided that this QUAL system adapted with an aptamer has the potential to act as a sensor and warrants further exploration.

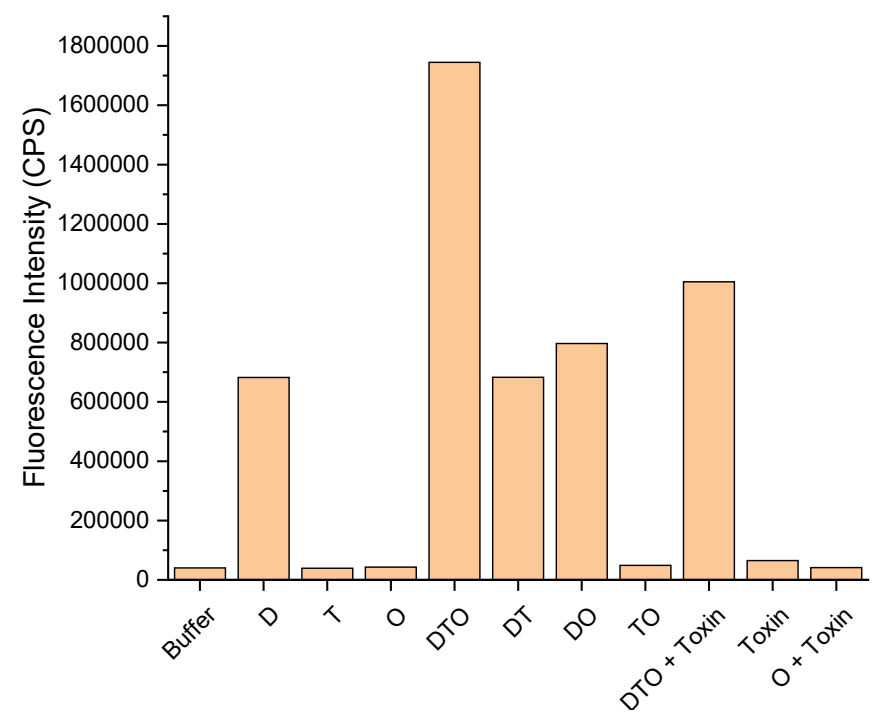

Figure 2.9: Bar graph of the data represented in Figure 2.8 at $520 \mathrm{~nm}$ emission demonstrating the ligation reaction between the dabsyl-fluorescein labeled probe $(D)$, thiophosphate probe $(T)$, and the complementary OTA aptamer $(O)$, in the presence and absence of $1 \mathrm{mM}$ OTA concentration. The fluorescence intensity of the reaction at $520 \mathrm{~nm}$ (DTO) is far greater than the comparable background, with the addition of toxin (DTO + Toxin) falling in between these two values as expected.

The QUAL system was analyzed over a series of fluorescence experiments to determine the correct concentration of the three probes and the correct reaction time to obtain optimal results. An optimal condition would result in a readily detectable signal difference between the reaction in the presence of toxin and in the absence of toxin, with a maximized difference in between these two conditions. It was determined that a 1-hour 
reaction time resulted in the maximum gap in fluorescence intensity between these two reactions. It was also determined that 10 micromolar DNA concentrations resulted in the largest increase in fluorescence signal from the reaction with minimal intermolecular reaction between the dabsyl and phosphorothioate probes in solution.

\subsubsection{Challenges associated with dabsyl lability and thiophosphate oxidation}

In the process of working with the QUAL system numerous challenges were encountered, some of which nearly spelled the end of this sensor system. The biggest issue that was encountered from the beginning was the limited stability of both the thiophosphate and dabsyl-fluorescein modified probes in storage and in reaction. The dabsyl probe could be synthesized with high yields, but due to the lability of the electrophilic dabsyl modifier decomposition was rapid and apparent. On standing, the dabsyl probe stability was estimated at under one week in solution before decomposition was observed. For this system, the decomposition of the dabsyl probe occurs through quencher loss, resulting in an increase in background fluorescence from the fresh product. If the background fluorescence increases significantly, as was observed on many occasions, the background signal would approach the maximum theoretical fluorescence of the ligated product. In these cases, the sensor capacity is limited, as the fluorescence of the system in the presence of toxin target will be in between the maximum fluorescence of the system in the absence of target and the background fluorescence of the dabsyl probe alone. This prompted extensive exploration of the decomposition products to verify quencher loss and strategies for stabilizing the probe. Mass spectrometry confirmed rapid quencher loss through loss of the dabsyl leaving group as a major contaminant in many of the samples, accounting for 
as much as half of the probe in solution in some cases (appendix Figure A.4). In the end, it was determined that new rapid cleanup strategies involving the Glen Pak cartridge with immediate use of the probe was the best course of action to limit the background signal and provide the greatest sensor capacity.

The thiophosphate probe was also observed to undergo a form of decomposition, in that dimerization of the probe through oxidation was observed in some cases. Though rare, dimerization of the thiophosphate probe did result in a significant decrease in reactivity in the system. Dimerization was demonstrated by molecular weight confirmation ESI-MS (appendix figure A.5). A strategy involving TCEP reduction of the thiophosphate probe was implemented along with storing in degassed buffer in a septum capped vial to eliminate contact with the oxygen in the air. This strategy was later eliminated, as it was found that the thiophosphate probe was sufficiently stable at $-80^{\circ} \mathrm{C}$ and that these extra steps were unnecessarily delaying the use of the more unstable dabsyl probe.

\subsubsection{Limit of detection and linear dynamic range assessment of ochratoxin A sensor system}

To evaluate the QUAL aptamer sensor system a wide range of ochratoxin A concentrations were tested. Testing was performed by incubating $10 \mu \mathrm{M}$ concentrations of each of the probes with a given concentration of ochratoxin A for one hour before measuring the fluorescence intensity relative to the reaction in the absence of ochratoxin A. It was expected that as the concentration of ochratoxin in the system decreases, the fluorescence intensity of the reaction mixture at 1 hour will approach the fluorescence 
intensity of the reaction in the absence of ochratoxin A. Therefore, higher concentrations of toxin will provide the lowest signal, while lower concentrations will give a higher signalresulting in a signal-off type sensor. As shown in Figure 2.10, the data appear to follow the expected trend for the reaction in the presence of toxin. In all reactions in which ochratoxin A was added along the dilution series between $100 \mu \mathrm{M}$ and $1 \mathrm{pM}$, the fluorescence intensity was found to be in between the reaction in the absence of toxin (DTO) and the background (D, DT, DO) as was expected.

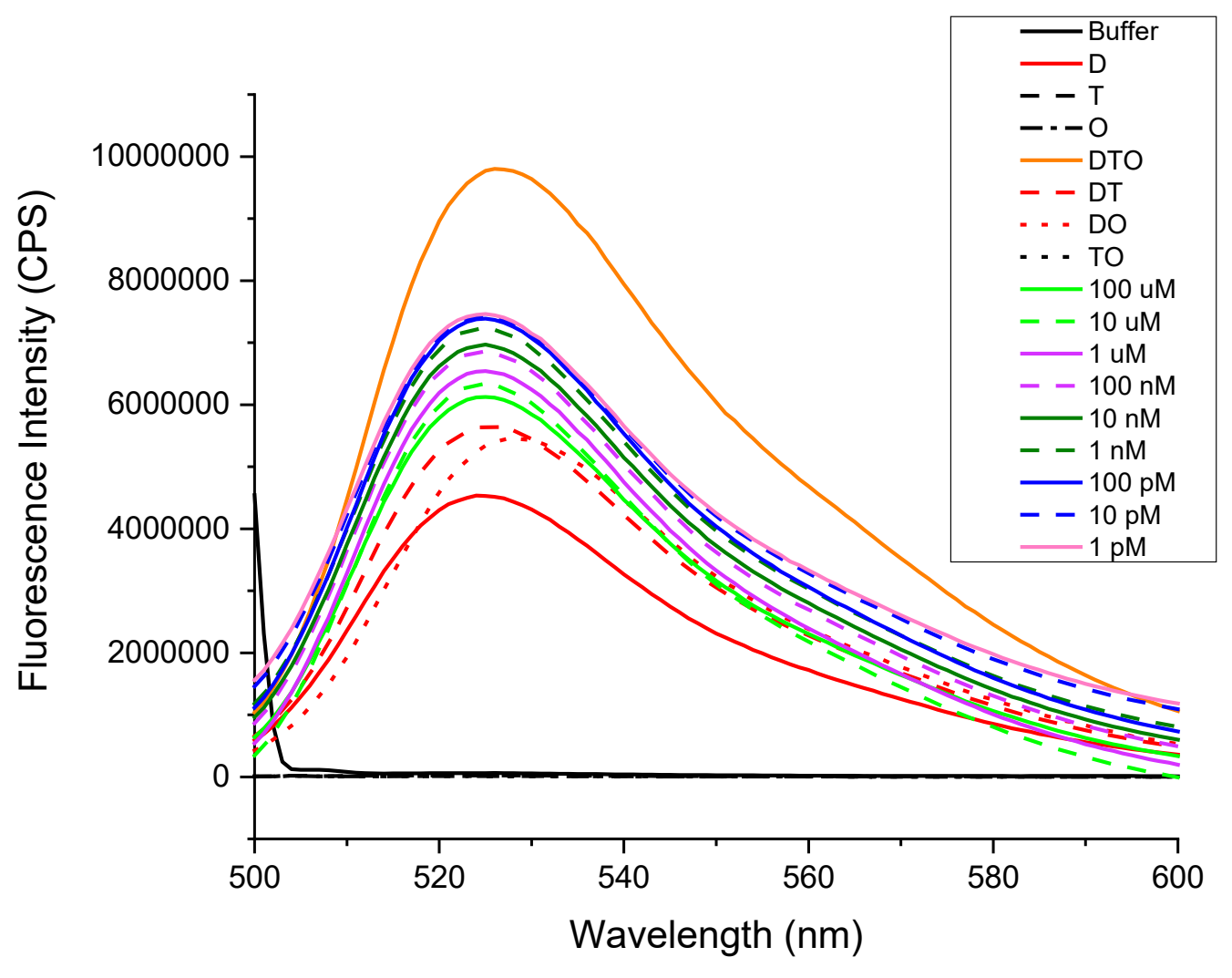

Figure 2.10: Fluorescence spectrum of the ligation reaction between the dabsyl-fluorescein labeled probe (D), thiophosphate probe (T), and the complementary OTA aptamer $(\mathrm{O})$, in the presence and absence of a range of ochratoxin A toxin concentrations $(100 \mu M-1 \mathrm{pM}) . \lambda_{\mathrm{ex}}=494 \mathrm{~nm}, \lambda_{\mathrm{em}}=500-600 \mathrm{~nm}$. The reaction (DTO) results in the highest fluorescence intensity, with the samples containing toxin 
falling below that in a trend of decreasing fluorescence with increasing ochratoxin A toxin concentration.

The trend in the emission at $520 \mathrm{~nm}$ from the fluorescein modifier was plotted in Figure 2.11. A one-way subjects ANOVA statistical analysis was performed in order to compare reported means in Figure 2.11, and it was found that at the $\alpha=0.05$ level, the population means are significantly different $[F(16,33)=6074.4, p=0]$. A two-factor means comparison following the ANOVA results using the Tukey test showed that at $\alpha=0.05$ the mean differences in the non-background related samples were significant except for those concentrations in the $100 \mathrm{pM}-1 \mathrm{pM}$ OTA concentration range, indicative of a plateau. Visual analysis shows that there is a trend in the fluorescence emission intensity in the presence of ochratoxin A, showing an apparent linear relationship between $100 \mu \mathrm{m}$ and $100 \mathrm{pM}$ with a plateau in the fluorescence intensity below $100 \mathrm{pM}$ toxin concentration. Interestingly, that plateau is occurring well below the fluorescence output of the reaction in the absence of toxin. It would be expected that low concentrations of toxin would result in similar fluorescence intensities to the reaction in the absence of toxin. This trend remains unexplained, but one possible explanation is that OTA itself has some form of quenching effect in solution and therefore its presence results in a decrease in fluorescence relative to the reaction (DTO). To determine if there was any quenching effect observed for ochratoxin A, a mixture of partially decomposed dabsyl probe was mixed with $1 \mu \mathrm{M}$ ochratoxin A and compared to the fluorescence emission of the probe in the absence of ochratoxin A in Figure 2.12. Addition of ochratoxin A results in a decrease in fluorescence intensity from the background dabsyl fluorescence in the partially decomposed probe, as 
well as a slight blueshift in the peak emission value. This is indicative of ochratoxin A having a minor quenching effect on the fluorescein emission from the dabsyl probe and warrants further exploration.

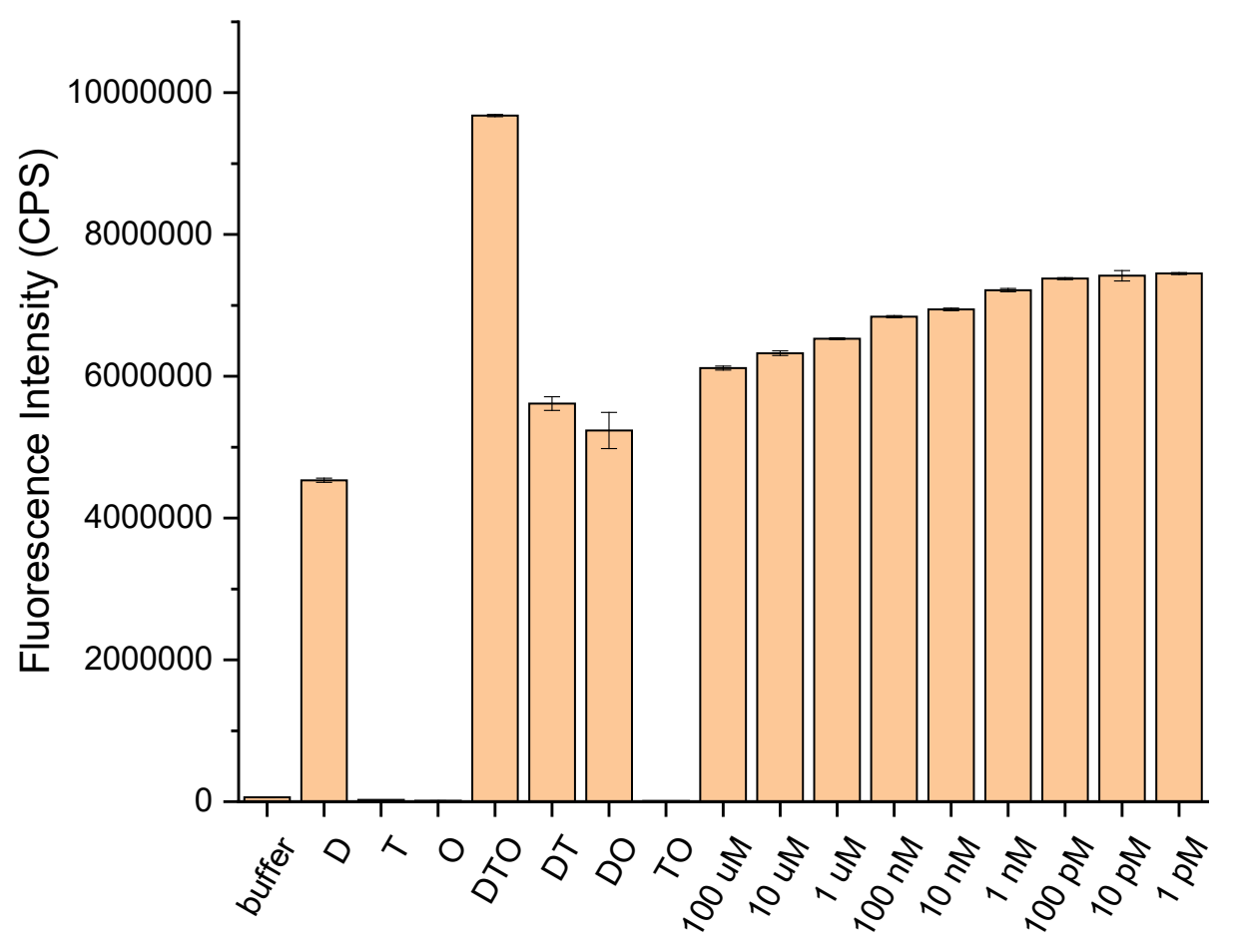

Figure 2.11: Bar graph of the data $(\mathrm{N}=3)$ represented in Figure 2.10 at $520 \mathrm{~nm}$ emission demonstrating the ligation reaction between the dabsyl-fluorescein labeled probe (D), thiophosphate probe (T), and the complementary OTA aptamer $(\mathrm{O})$, in the presence and absence of a range of ochratoxin A toxin concentrations $(100 \mu \mathrm{M}-1 \mathrm{pM})$. A linear trend in the fluorescence intensity of the reaction in the presence of toxin is observable between $100 \mu \mathrm{M}$ and $100 \mathrm{pM}$ after which is plateaus, with all reactions in the presence of toxin having lower fluorescence output than the standard reaction (DTO). One way subjects ANOVA at $\alpha=0.05$ showed a significant difference between all means $[F(16,33)=6074.4, p=$ 0]. 


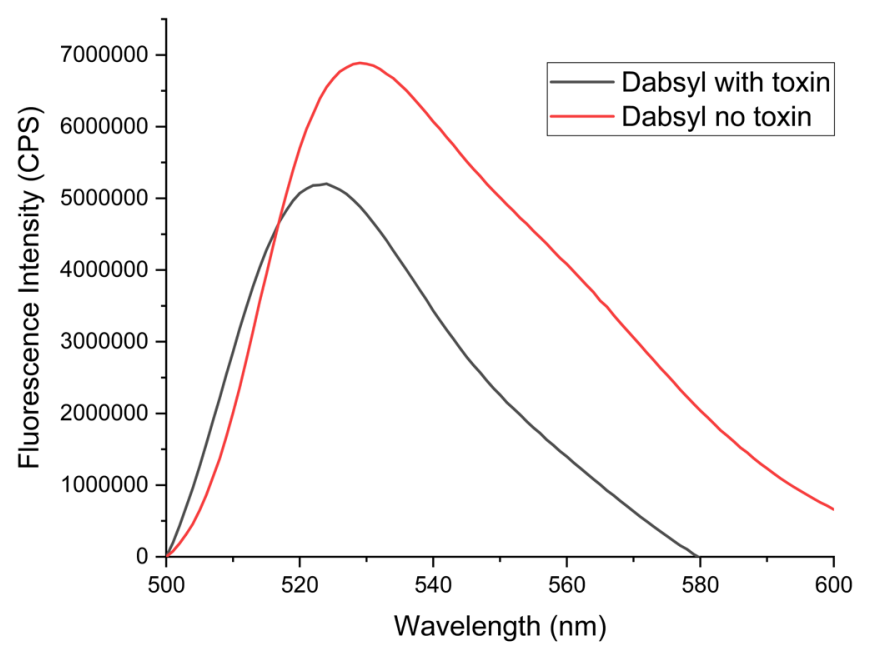

Figure 2.12: Fluorescence emission spectrum of dabsyl probe incubated with and without ochratoxin $A$ at $1 \mu \mathrm{M}$ concentration. The addition of toxin appears to quench the fluorescence emission of the dabsyl probe and result in a slight blueshift of the peak.

Converting the Figure 2.11 into a scatter plot permitted an assessment of the linear dynamic range of the system, which was shown to be over the range of $100 \mu \mathrm{M}$ to $100 \mathrm{pM}$ as seen in Figure 2.13, with a $100 \mu \mathrm{M}$ limit of linearity. This system could therefore be used for the quantification of ochratoxin A over 6 orders of magnitude. Though no regulations on the levels of ochratoxin A in foodstuffs currently exist in Canada, the lowest limit seen in the European Union is $3 \mu \mathrm{g} / \mathrm{kg}$, or $3 \mathrm{ppb} .^{113-115}$ When converted to molarity this is equivalent to an ochratoxin A concentration of $7.4 \mathrm{nM}$, well within the linear range of the proposed sensor.

The QUAL based ochratoxin A sensor compares favourably to other reported sensors in literature. Recent developments in label-free aptamer or fluorescent aptamer sensors have reported linear dynamic ranges of $2.5 \mathrm{nM}$ to $250 \mu \mathrm{M}$ and $20 \mathrm{nM}$ to $100 \mathrm{nM}$ respectively. ${ }^{121-122}$ The label-free aptamer sensor reaches 5 orders of magnitude of linearity 
compared to the 6 orders reported herein for the QUAL sensor. Surface plasmon-resonance based aptasensors have also been developed for ochratoxin A with a linear dynamic range of $10 \mathrm{pM}$ to $100 \mathrm{nM}{ }^{123}$ Electrochemical aptamer sensors have been reported with linear dynamic ranges that fall far below the values reported herein, with ranges reported as low as $0.125 \mathrm{pM}$ to $1 \mathrm{pM}$ ochratoxin A. ${ }^{124}$ While important for the development of sensor methodology, these do not have significant validity in the field as the sensor should be able to detect in the area surrounding the regulatory limit of $7.4 \mathrm{nM}$ and above. Being able to detect below this range is not as important for detecting high levels of a toxic substance in foodstuff. This demonstrates the importance of the QUAL method reported herein, as the wide linear dynamic range covers from well below the regulatory limit to well above it, allowing for a more useful sensor in applications involving food especially in potentially contaminated crops where quantification is essential. 


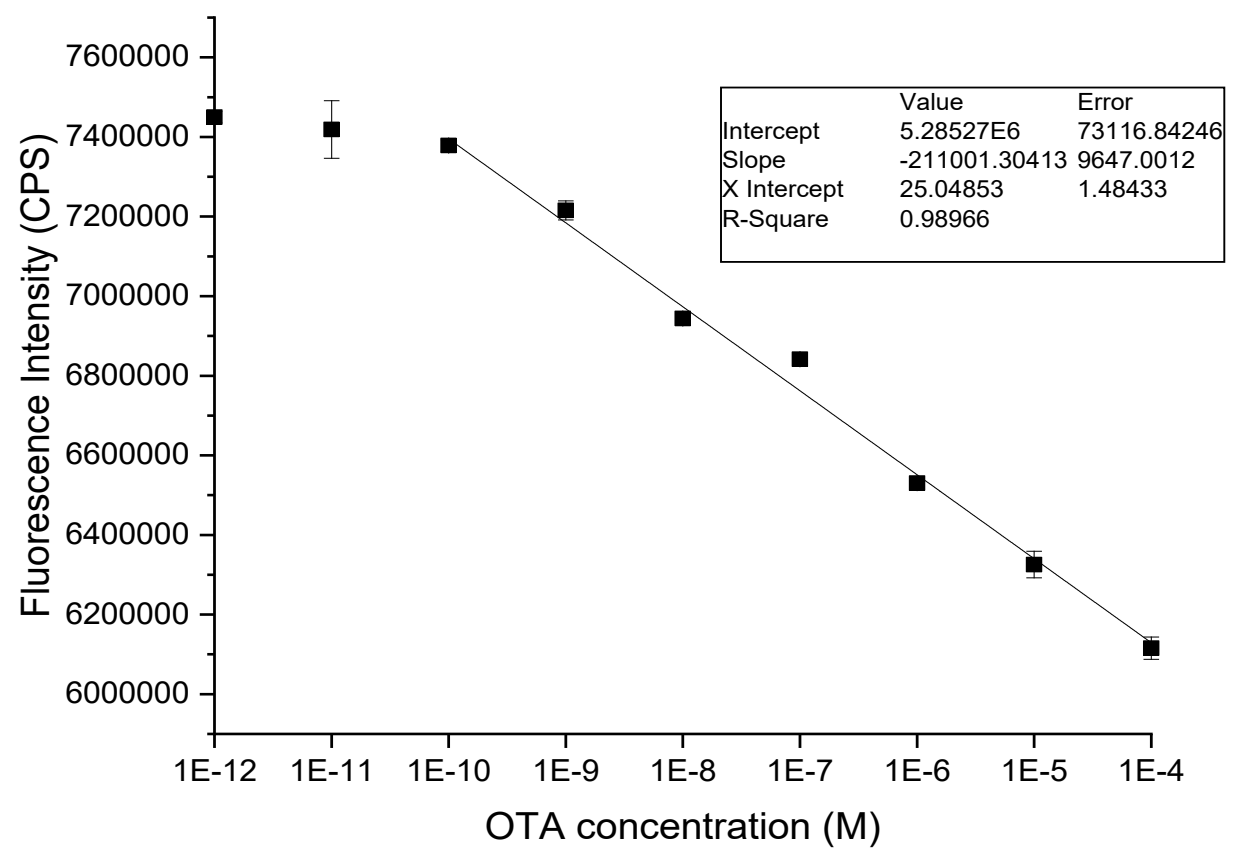

Figure 2.13: Scatter plot of the observed trend at $520 \mathrm{~nm}$ emission for the reaction in the presence of $100 \mu \mathrm{M}$ to $1 \mathrm{pM}$ ochratoxin A concentrations. A linear trend in fluorescence intensity with toxin concentration is observed between $100 \mu M$ and $100 \mathrm{pM}$, with an $R^{2}$ value of 0.989 . All data points were run in triplicate $(\mathrm{N}=3)$, with error bars for some concentrations hidden by the markers.

Evaluation of the limit of detection of the QUAL aptamer system was performed using the standard deviation of the reaction in the absence of ochratoxin A (DTO). By plotting the difference in the fluorescence intensity between the reaction in the absence of OTA and at a given concentration, a linear trend was observed when plotted against the logarithm of OTA concentration in $\mathrm{pM}$, much like that seen in Figure 2.13. Using the equation of the line shown in Figure 2.14, the limit of detection can be calculated as the concentration at which the fluorescence intensity is equal to 3 times the standard deviation of the buffer fluorescence. ${ }^{125}$ Inputting those numbers, an LOD of $1.5 \mathrm{pM}$ can be 
calculated. The limit of quantification can also be calculated, using 10 times the standard deviation of the buffer fluorescence, as $4.5 \mathrm{pM}$.

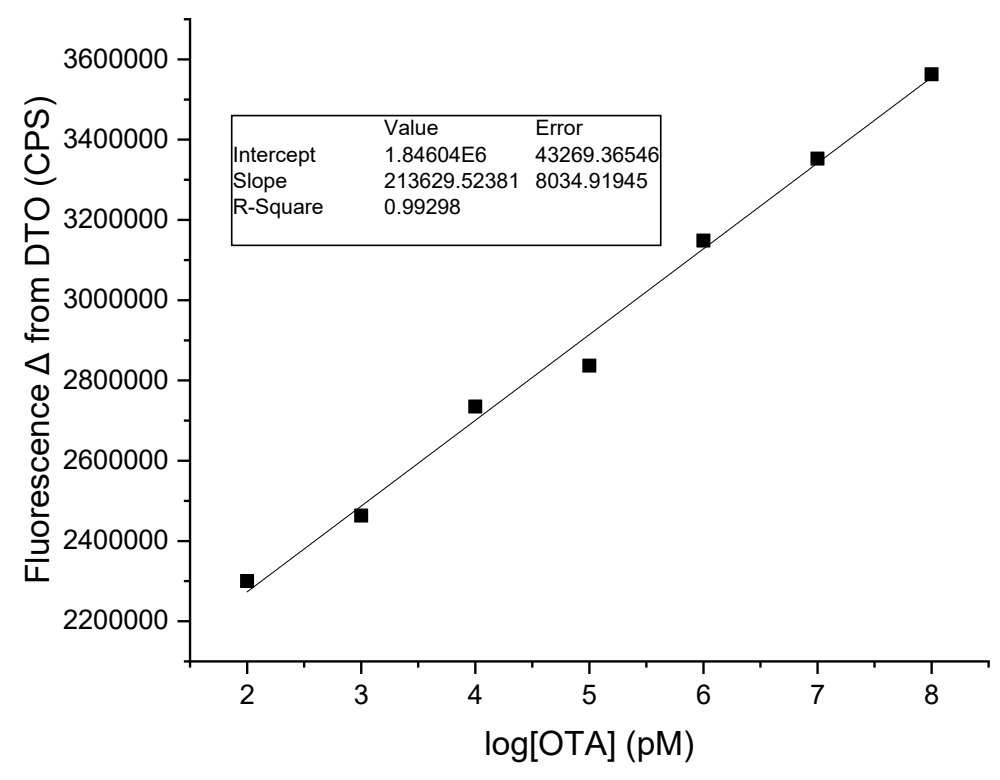

Figure 2.14: Plot of change in fluorescence from DTO for each ochratoxin A concentration, plotted against the logarithm of ochratoxin A concentration in $\mathrm{pM}$. Using the regression analysis, an LOD of $1.5 \mathrm{pM}$ was calculated. All data were run in triplicate $(\mathrm{N}=3)$, with some error bars hidden by markers.

The limit of detection for the QUAL sensor is quite promising when compared to other aptamer-based sensors and the currently used methods of detecting ochratoxin A. The limit of detection is the lowest amount of ochratoxin A that could be detected using the sensor at hand. ${ }^{126}$ Current LC fluorescence methods for the detection of ochratoxin A can detect levels above $5 \mathrm{nM}$, which is just below the regulatory limit in the European Union. Fluorescent and label free aptasensors have been developed with limits of detection from as low as $2.5 \mathrm{pM} .^{121-122}$ Electrochemical based methods show far greater sensitivity, with limits of detection as low as $25 \mathrm{fM}$ ochratoxin A. ${ }^{124}$ Fluorescence based assays will 
typically be much lower sensitivity than optical and electrochemical based methods, but the ability to detect down to $1.5 \mathrm{pM}$ constitutes one of the most sensitive aptamer-based sensors that does not rely on one of these more complex setups. Therefore, the QUALbased system can be considered a success when compared to other aptamer-based systems for both the wide linear dynamic range that it provides and the low limit of detection.

\subsubsection{Controls and specificity of the ochratoxin A sensor system}

To fully characterize the sensor, an evaluation of the specificity of the reaction was performed. The fluorescence intensity of the reaction in the presence of thrombin, and adenosine was compared to the fluorescence intensity of the reaction in the absence of toxin (DTO). It is expected that the addition of a molecule that is not the target of the aptamer used in the system will result in the fluorescence intensity being approximately the same as the reaction in the absence of toxin. Figure 2.15 and Figure 2.16 demonstrate that the addition of thrombin and ATP results in approximately the same fluorescence as the DTO reaction as expected. Replacing the aptamer used in the reaction also had a significant effect on the fluorescence output. By replacing the OTA aptamer with a thrombin aptamer, the ligation reaction did not occur even in the presence of $10 \mu \mathrm{M}$ OTA toxin. Therefore, the reaction is specific to the OTA aptamer and its target. 


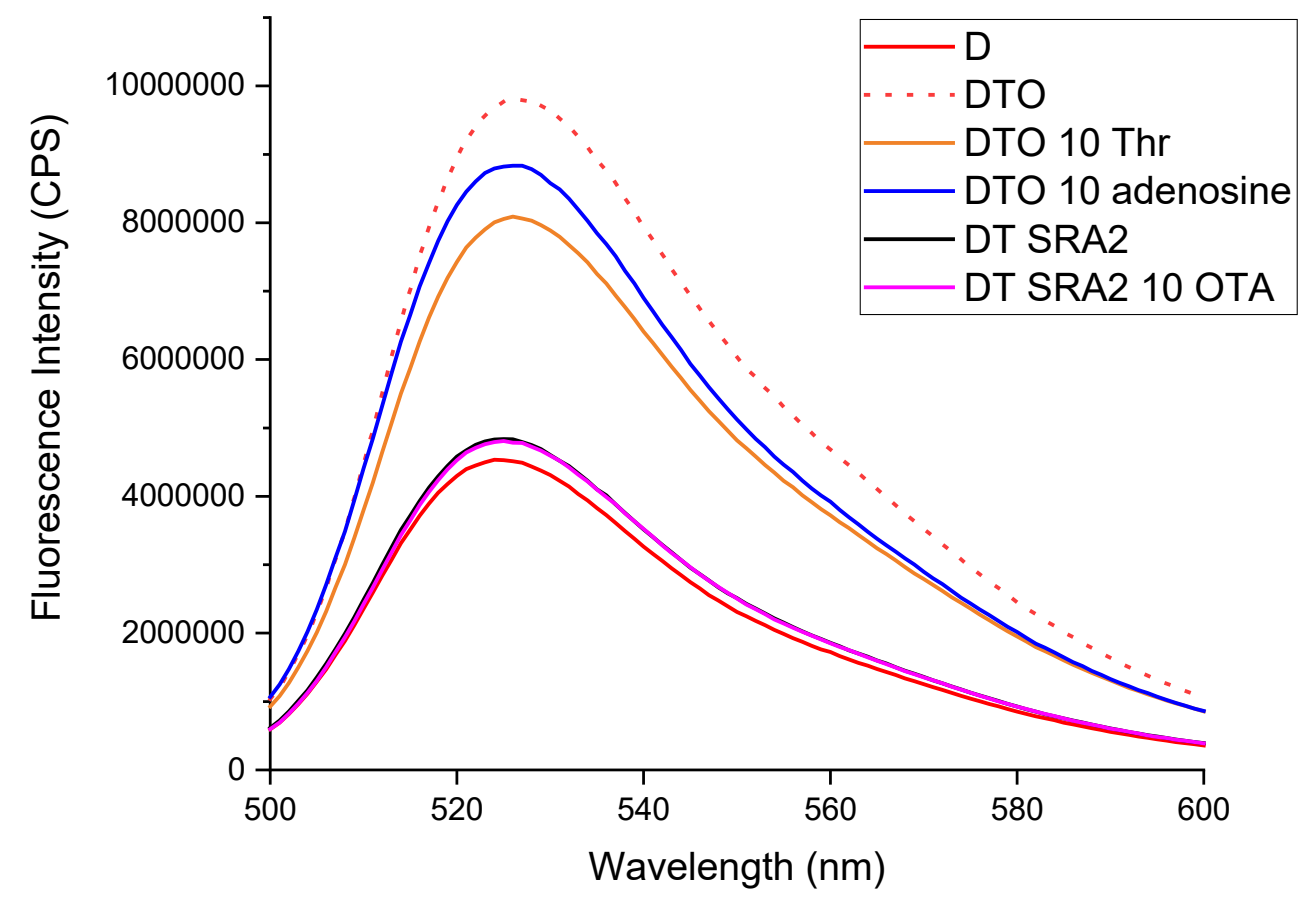

Figure 2.15: Fluorescence spectrum of the ligation reaction between the dabsyl-fluorescein labeled probe (D), thiophosphate probe (T), and the complementary OTA aptamer $(\mathrm{O})$, in the presence of 10 $\mu \mathrm{M}$ concentration of adenosine, ATP, and thrombin as controls. The OTA aptamer was also replaced with the thrombin SRA_2 aptamer. $\lambda_{\mathrm{ex}}=494 \mathrm{~nm}, \lambda_{\mathrm{em}}=500-600 \mathrm{~nm}$. The addition of thrombin and adenosine results in a slight decrease in fluorescence emission in the DTO system, while replacement with the SRA_2 aptamer results in no signal difference from background dabsyl (D). 


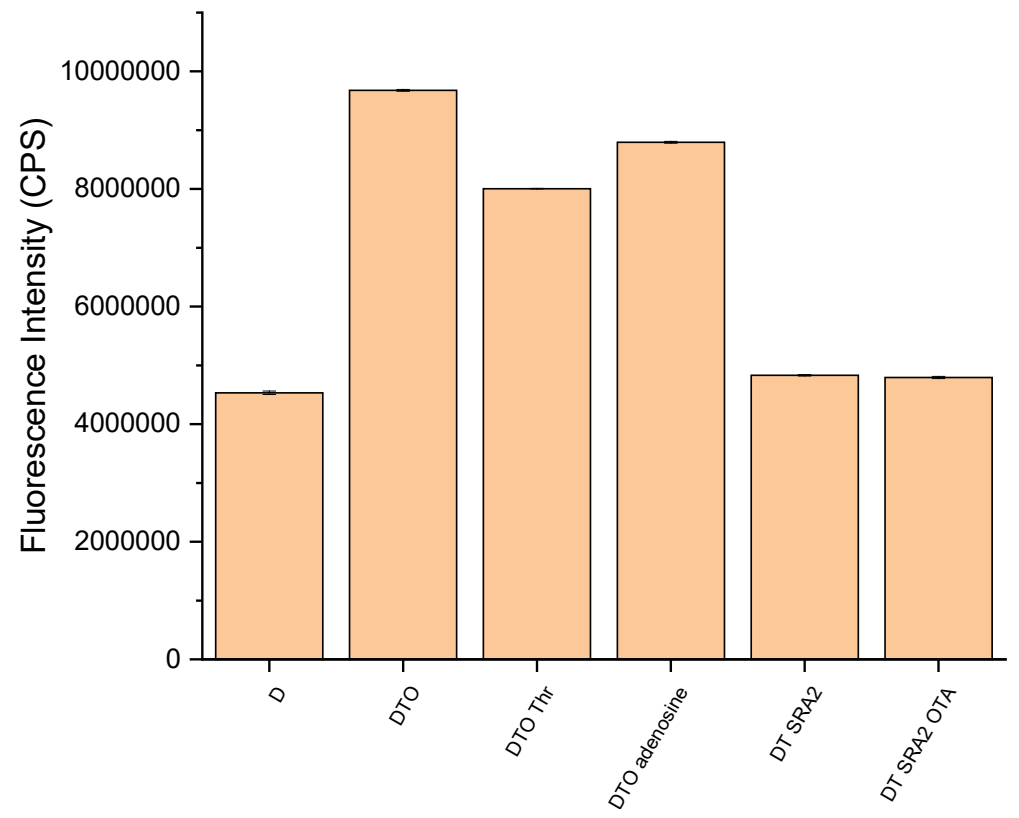

Figure 2.16: Bar graph of the data represented in Figure 2.15 demonstrating the ligation reaction between the dabsyl-fluorescein labeled probe (D), thiophosphate probe (T), and the complementary OTA aptamer $(\mathrm{O})$, in the presence $10 \mu \mathrm{M}$ concentration of adenosine, ATP, and thrombin as controls. The OTA aptamer was also replaced with the thrombin SRA_2 aptamer. All data were run in triplicate $(\mathbf{N}=\mathbf{3})$.

\subsection{Conclusions}

The development of an ochratoxin A sensor in analogy to the previously demonstrated quenched autoligation probe system of Kool proved successful. This constitutes one of the first demonstrations of an aptamer controlling a chemical ligation reaction between two sequences which can be inhibited through the target of the aptamer. This is also the first fluorogenic sensor based on strand ligation controlled by an aptamer and its target. This expands the potential for aptamer technology not only in the field of sensor development, but also in other related fields where the control of a chemical reaction 
may need to be spatiotemporally controlled. Molecular payload delivery could become one future application of these spatiotemporally controlled reactions, wherein aptamertarget binding could be used to control the release of a small molecule. This small molecule could be a drug for example, and its controlled release would result in fewer non-specific effects. If the aptamer target were to denote a healthy cell as opposed to a diseased cell, a system such as that designed herein could be used to turn off the reaction and therefore avoid non-specific killing of healthy cells.

The system designed herein had a linear dynamic range of $100 \mu \mathrm{M}$ to $100 \mathrm{pM}$, with a limit of detection of $1.5 \mathrm{pM}$. This is a valid system for the currently controlled levels of ochratoxin A in foodstuffs. This system was also shown to have specificity for its target, as well as for the aptamer used in the system.

This system is not without its challenges. Rapid decomposition of the dabsyl probes causes significant problems in regulating the background and induces substantial variability in between QUAL tests with toxin. This reaction is also fluorogenic, which while sensitive is not practical for helping with simplified in field tests. Future consideration of chromogenic reactions for rapid in-field assessment of toxin presence could be the next avenue for such a system. This system can also be expanded to other comparable targets with known structure switching aptamers in the future to expand the scope of QUAL sensor systems. 


\section{Chapter: Synthesis of modifiers for fluorogenic click templated chemical reactions using DNA}

\subsection{Statement of contributions}

Christopher Mattice performed experimental design and synthetic work towards the synthesis of the alkyne-coumarin phosphoroamidite.

\subsection{Introduction}

\subsubsection{Click chemistry}

The term "click chemistry" was proposed by Barry Sharpless and coworkers in 2001 to describe a set of logistical chemical steps that were inspired by nature. These chemical steps would be useful in the synthesis of molecules, small or large, particularly as it applied to the pharmaceutical industry. ${ }^{127}$ The thought at the time was that drug companies and their employed synthetic chemists were becoming too focused on structure over function, and in many ways, this was leading to detrimental effects on the industry, due to complex and inefficient syntheses and the added development time. ${ }^{127-128}$ By restricting structure-activity relationship (SAR) searches to molecules that were easy to make by simplifying the chemistry, an improvement should be seen in drug development costs and the field of synthetic organic chemistry. ${ }^{128}$

By joining small units together via heteroatom links, as is common in the condensation reactions in nature (polynucleotides, polypeptides, and polysaccharides), a set of reactions based on modular blocks can be outlined. ${ }^{129}$ These reactions would 
constitute the field of click chemistry, and the stringent criteria for a reaction to be considered a click reaction were outlined by Sharpless in $2001:{ }^{127}$

- Modular, and give very high yields

- Generate only inoffensive byproducts that can be easily removed without chromatography

- Be stereospecific (enantioselectivity is not a requirement)

- Simple reaction conditions from readily available starting materials and reagents

- The use of no solvent or benign solvent such as water, or a solvent that can be easily removed

- Simple product isolation, with purification being non-chromatographic

There are a variety of reactions that satisfy these requirements, with the first class of reactions being nucleophilic ring opening reactions- specifically as they apply to springloaded three-membered rings, such as epoxides and aziridines, but have also been applied to cyclic sulfates and episulfonium ions as shown in Figure 3.1. ${ }^{129}$ High yields are ensured since the competing elimination reactions are stereoelectronically disfavored. Such reactions are often used to install click handles such as azides for subsequent reactions.

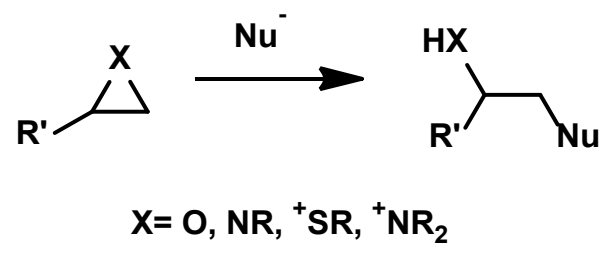

Figure 3.1: General reaction scheme for nucleophilic ring opening reactions as seen in click chemistry. 
Additions to carbon-carbon multiple bonds, such as alkenes, can also be classified as click reactions; examples include epoxidations, aziridinations, dihydroxylations, and sulfenyl halide additions. ${ }^{129}$ Thiol click reactions are one of the most prevalent examples of this addition and are commonly used in the field of polymer chemistry - though disadvantages related to the orthogonality of sulfur addition exist, limiting its applicability. ${ }^{129-130}$ Thiol addition can either proceed through a radical reaction or a Michael addition, with the latter resulting in fewer byproducts and better orthogonality than the former.

a)

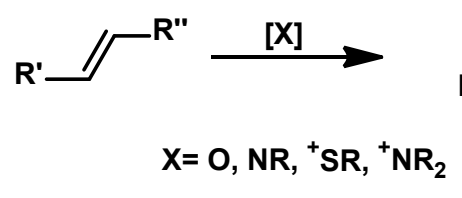

b)

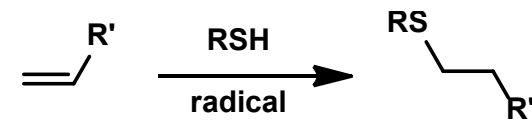

c)

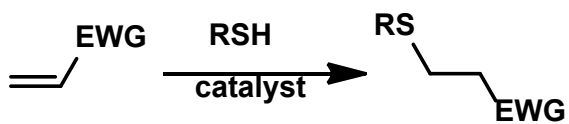

Figure 3.2: General reaction schemes for the addition to carbon-carbon multiple bond class of click reactions. Examples of these reactions include a) addition across an alkene to form a strain three member ring system, b) thiol addition under radical conditions, and c) thiol addition to a Michael acceptor.

The formation of oxime-ethers, hydrazones, and aromatic heterocyles from carbonyls in non-aldol type reactions are also categorized as click reactions. ${ }^{129}$ The aldol reactions could not be classified as click reactions due to their low thermodynamic driving force. ${ }^{127}$ Hydrazone formation from the reaction of an aldehyde or ketone with hydrazide is of interest in many fields due to the reversibility of the linkage, unlike the more stable oxime. $^{129}$ 


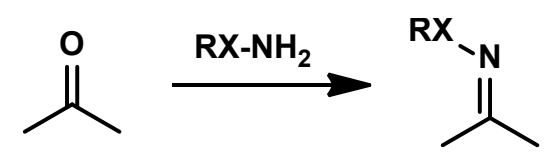

$X=0, N^{\prime}$

Figure 3.3: General scheme of the non-aldol carbonyl chemistry reaction class of click chemistry, depicting the formation of either oximes $\mathrm{X}=\mathrm{O}$, or hydrazides $\mathrm{X}=\mathrm{NR}$.

The final category of click reactions are cycloaddition reactions, which can be further subdivided into Diels-Alder reactions and 1,3-dipolar cycloaddition reactions. These reactions link two unsaturated reactants together without breaking any $\sigma$-bonds in a fusion type reaction to generate five- and six-membered heterocycles. Diels-Alder reactions (DA) typically involve the formation of a stable six-membered rings through a [4+2] cycloaddition, typically between an electron-rich diene and an electron-poor dienophile. ${ }^{130-131}$ 1,3-Dipolar cycloadditions are most commonly demonstrated with Huisgen cycloadditions, which is a cycloaddition between an azide and an alkyne to generate a 1,4 - or a 1,5-substituted triazole. ${ }^{129,132}$ Huisgen cycloadditions are considered the best example of click chemistry reactions and have in most ways taken over the "click chemistry" term in the scientific community, though all the reactions detailed herein are considered click chemistry. ${ }^{129}$ Huisgen cycloadditions, and specifically copper catalyzed azide-alkyne cycloadditions (CuAAC) will be discussed in greater detail in the subsequent sections. 
a)

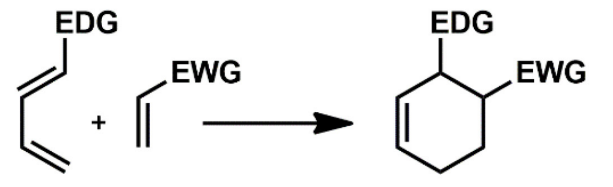

b)

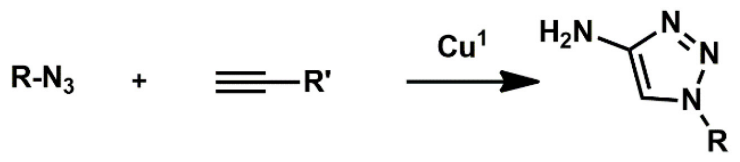

Figure 3.4: Cycloadditions occupy an important spot in click chemistry, with the two most prevalent examples being the a) Diels-Alder cycloaddition between an electron rich diene and electron poor dienophile to generate a six-membered ring, and b) the azide-alkyne cycloaddition to produce a fivemembered ring triazole

\subsubsection{Azide-Alkyne cycloadditions}

The 1,3-dipolar cycloaddition of azides and alkynes to afford 1,2,3-triazoles has quickly become the most powerful example of a click reaction, with the copper-catalyzed variant being the most prevalent. In general, both azides and alkynes are easily incorporated into molecules, then remain stable under most conditions and generally orthogonal to most chemistry. ${ }^{127}$ This is particularly relevant when azides and alkynes are incorporated into biological systems, or in many sensor development applications where extraneous reactions must be avoided. ${ }^{133}$ As was initially reported by Huisgen, both azides and alkynes have a high level of kinetic stability, which results in slow uncatalyzed cycloaddition. $^{129,}$ 134-135 These uncatalyzed reactions (Figure 3.5), require extended reaction times and high temperatures, which makes them unsuitable for click chemistry. ${ }^{127-}$

128 These reactions also tend to suffer from poor-regioselectivity, resulting in the formation of both 1,4 and 1,5 regioisomers of the triazole product. ${ }^{134}$ 


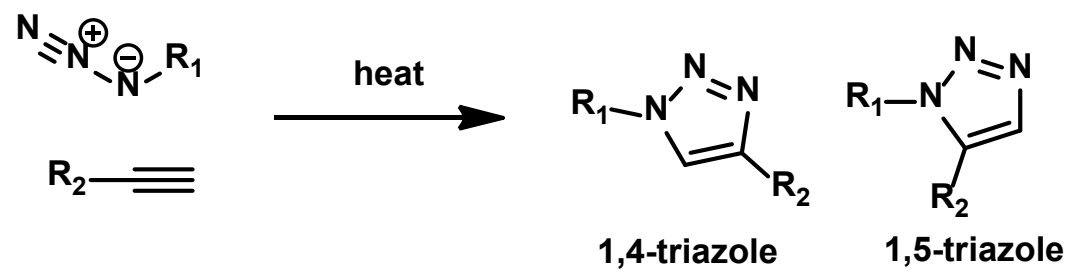

Figure 3.5: Uncatalyzed thermal 1,3-cycloadditon between an azide and an alkyne results in a 1:1 ratio of 1,4-:1,5-substituted triazole.

The Sharpless and Meldal groups demonstrated in 2002 that the azide-alkyne cycloaddition could be accelerated (up to $10^{7}$ rate enhancement) and made relevant to click chemistry through the addition of a $\mathrm{Cu}(\mathrm{I})$ catalyst. ${ }^{136-138}$ The copper catalyst also enhances regioselectivity, unlike the uncatalyzed variant, with the 1,4-regioisomer being the exclusive product. ${ }^{134,139}$ The reaction functions in a variety of solvents (including water), at a wide range of temperatures, and is unaffected by steric factors on the azide or alkyne. ${ }^{136}$ Of particular relevance to its biocompatibility, the triazole formed is a bioisostere of an amide bond (Figure 3.6b). ${ }^{139}$

a)

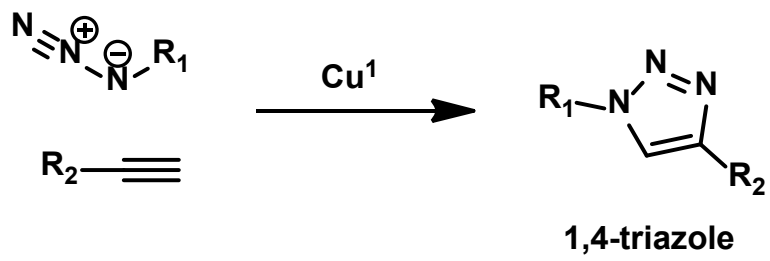

b)

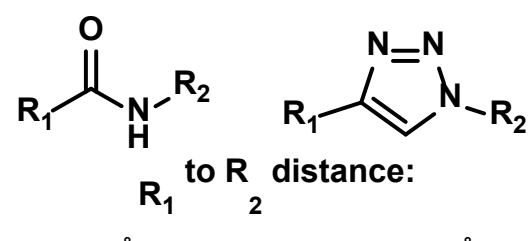

$3.9 \AA$

Figure 3.6: a) The copper(I) catalyzed azide-alkyne cycloaddition results in the rapid production of solely the 1,4-triazole regioisomer. b) The triazole has similar $R_{1}$ to $R_{2}$ distance to an amide bond.

Mechanistically, the copper-catalyzed azide-alkyne cycloaddition is thought to occur in a stepwise fashion, as opposed to the concerted mechanism seen in most cycloaddition reactions. In 2005, the stepwise mechanism was proposed based on kinetic 
data and is depicted in Figure 3.7. ${ }^{140-141}$ The calculated activation barrier for the concerted reaction between an azide and a copper-acetylene $\pi$-complex is $23.7 \mathrm{kcal} / \mathrm{mol}$, which is higher than the calculated activation energy for the uncatalyzed reaction. ${ }^{136}$ Stepwise cycloaddition catalyzed by a monomeric $\mathrm{Cu}(\mathrm{I})$ species showed a much lower activation barrier of $11 \mathrm{kcal} / \mathrm{mol}$, although it has since been shown that the reaction is at least second order with respect to $\mathrm{Cu}(\mathrm{I}){ }^{136}$ Typical architectures of the $\mathrm{Cu}(\mathrm{I})$-alkyne complexes were found to show each $\mathrm{C}-\mathrm{C}$ triple bond coordinating either 2 or $3 \mathrm{Cu}$-atoms with bond angles in the $130-140^{\circ}$ range, with very few existing with a single end-on coordinated $\mathrm{Cu}$ atom with a bond angle of $180^{\circ} .{ }^{134,136}$ It is for this reason that it has been postulated, and still not conclusively proven, that the azide and alkyne are not coordinated to the same copper atom in the transition state, leading to a transition state like structure B in Figure 3.7. ${ }^{136}$ 


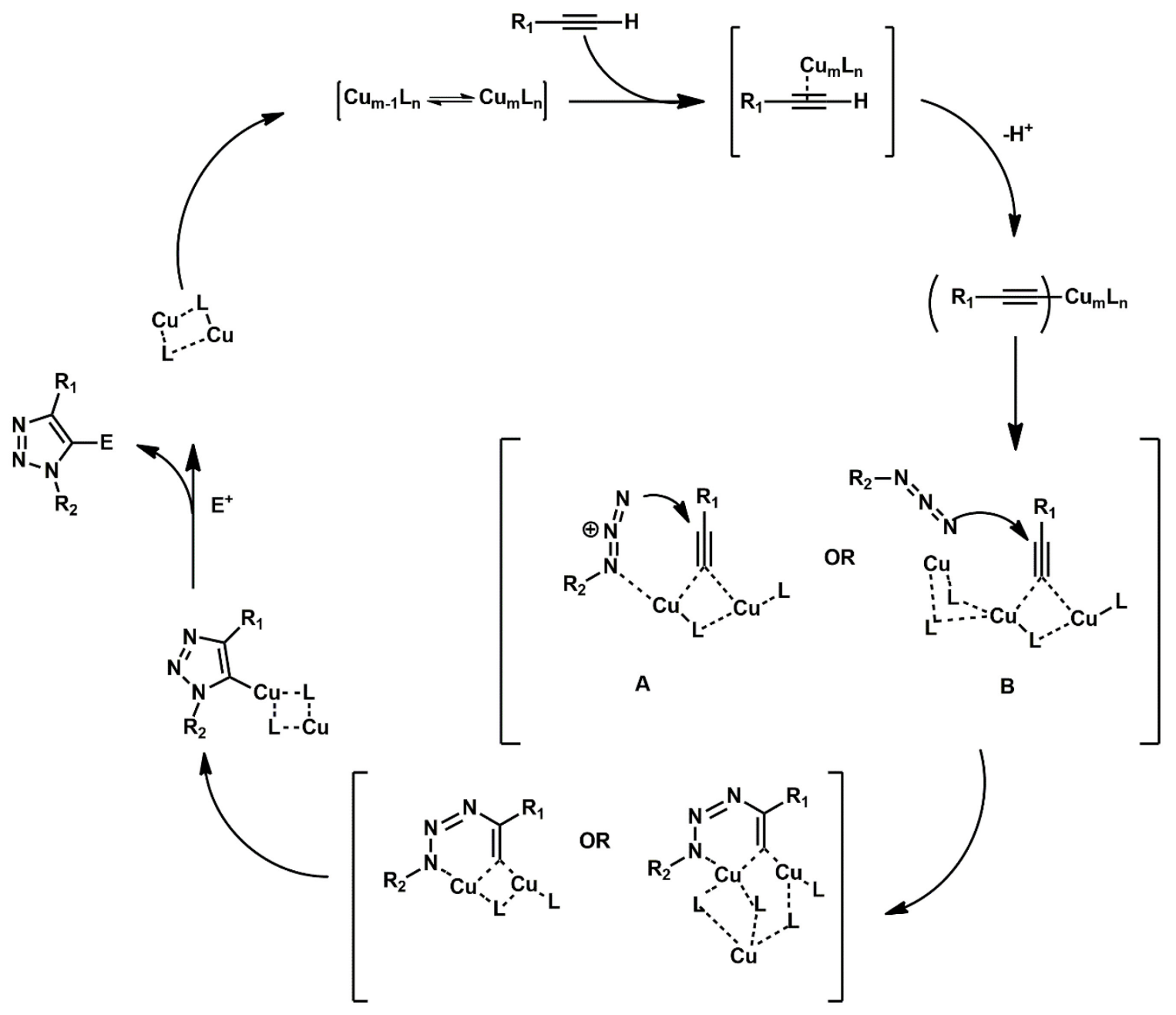

Figure 3.7: Proposed scheme for the copper-catlyzed azide-alkyne cycloaddition. Two possible transition states are possible, as depicted by $A$ and $B$, involving either two or three copper atoms respectively.

A variety of $\mathrm{Cu}(\mathrm{I})$ sources have been used in the literature, with the most prevalent being the use of a $\mathrm{Cu}(\mathrm{II})$ species such as copper sulfate, along with a reducing agent such as sodium ascorbate or tris(2-carboxyethyl)phosphine (TCEP) to generate the requisite $\mathrm{Cu}$ (I) functionality in solution. ${ }^{136}$ Other common copper sources include $\mathrm{CuI}$ and $\mathrm{CuBr}$, and in some cases $\mathrm{Cu}(0)$ species such as copper wire or nanoparticles. Ligands can also be added to the reaction mixture, though they are not required for the catalytic effect of $\mathrm{Cu}(\mathrm{I})$, 
and are generally added to enhance the rate of the reaction and to protect $\mathrm{Cu}(\mathrm{I})$ species from oxidation to $\mathrm{Cu}(\mathrm{II}) .{ }^{136,139}$ Common accelerating and protecting ligands used are the polytriazoles TBTA and its water-soluble analogue THPTA (Figure 3.8). ${ }^{142}$ It has been shown that both ligands can protect $\mathrm{Cu}(\mathrm{I})$ under aerobic conditions, and were postulated to work via the tertiary amine donating electron density into the copper centre, while the latter, being more labile, comes off the $\mathrm{Cu}(\mathrm{I})$ temporarily to allow formation of the copper(I)-acetylide/ligand complex. ${ }^{139,142}$

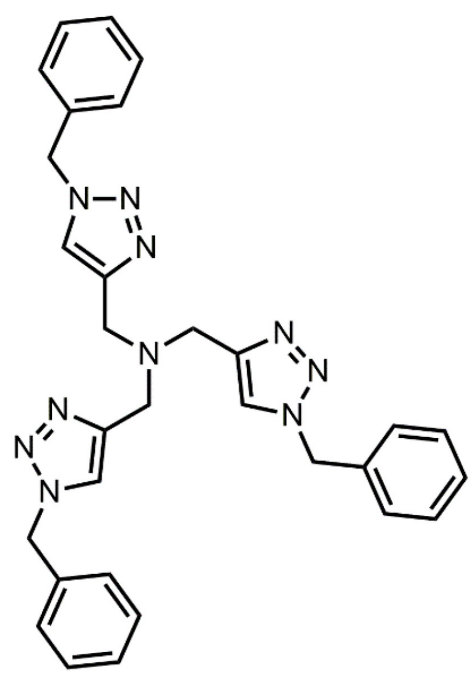

TBTA

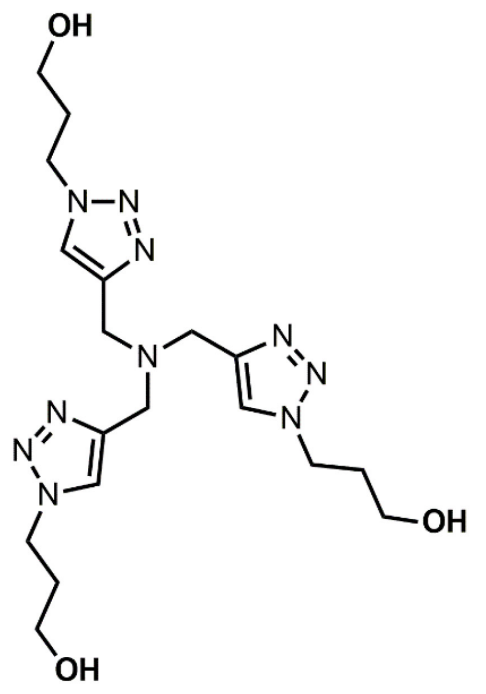

THPTA

Figure 3.8: Strictures of the common catalytic triazoles used in copper catalyzed azide-alkyne cycloadditions, showing tris[(1-benzyl-1H-1,2,3-triazol-4-yl)methyl]amine (TBTA), and its water soluble analog tris(3-hydroxypropyltriazolylmethyl)amine.

\subsubsection{Copper-free click chemistry}

In the early 2000s, a new field of click chemistry was created based on the need for bioorthogonal reactions as a toolset for labeling in chemical biology. The azide tag had long been established as a bioorthogonal tag for in vivo labeling experiments, but at the 
time the only truly compatible reaction was the Staudinger ligation as demonstrated by the Bertozzi lab. ${ }^{143}$ It suffered from issues related to the requisite phosphines and their stability, and the competing copper-catalyzed Huisgen reaction suffered from the toxicity of $\mathrm{Cu}(\mathrm{I})$ species in biological systems. ${ }^{133,144}$ This led to the creation of the field of strainpromoted azide-alkyne cycloadditions between cyclooctynes and azides in the absence of catalysts by the Bertozzi lab in 2004. ${ }^{133}$ The chemistry was based on the work of Wittig and Krebs in the 1960s, where it had been demonstrated that the reaction of the smallest stable cycloalkyne, cyclooctyne with its large bond angle deformation of $163^{\circ}$, reacted explosively with phenyl azide without catalysts to generate a triazole. ${ }^{133}$ In a 2008 study of the energetics of the strained reaction by Ess et al., it was demonstrated that the difference in reactivity for the strain-promoted reaction relative to the reaction of acetylene and phenyl azide was a result of the energy required to distort the 1,3-dipole of the azide to form the transition state, rather than a molecular orbital interaction. ${ }^{145}$ The N-N-N bond angle for the acetylene reaction was on the order of $138^{\circ}$, relative to the ground state of $173^{\circ}$, while that in the reaction of cyclooctyne was $142^{\circ}$. This results in an energy barrier of $16.2 \mathrm{kcal} / \mathrm{mol}$ for the acetylene/phenyl azide reaction, compared to $8.0 \mathrm{kcal} / \mathrm{mol}$ for the cyclooctyne/phenyl azide reaction. ${ }^{145}$

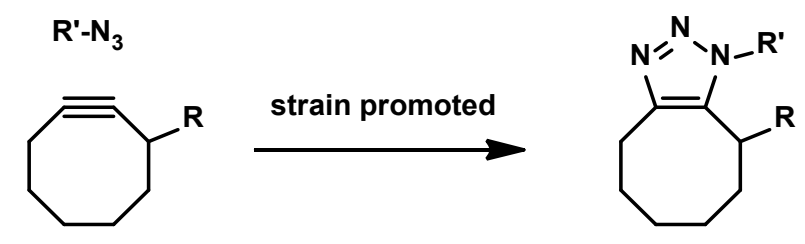

Figure 3.9: The strain promoted azide-alkyne cycloaddition occurs between a strained cyclootyne ring and an azide to produce a triazole in the absence of a copper catalyst. 
While effective, the rate of the first-generation strain promoted azide-alkyne cycloaddition reagents did not exceed that of the standard Staudinger reaction, and therefore alternative alkyne reagents were explored by Bertozzi. To increase the rate of the reaction, two rate enhancing features were incorporated into the copper-free reaction at once, one being the additional ring strain afforded by the cyclooctyne, and the other being electron-withdrawing groups. ${ }^{130,146}$ The choice of electron withdrawing groups in this case were difluoro- and monofluoromethylene moieties, called DIFO and MOFO respectively. These electron-withdrawing groups were chosen due to their biocompatibility, and the fact that they do not introduce an additional $\pi$-conjugated substituent, which could result in Michael addition reactions. ${ }^{146}$ MOFO provided a 2-fold rate enhancement, while DIFO provides a rate enhancement of up to 30 times over previous strain promoted reactions. ${ }^{130}$

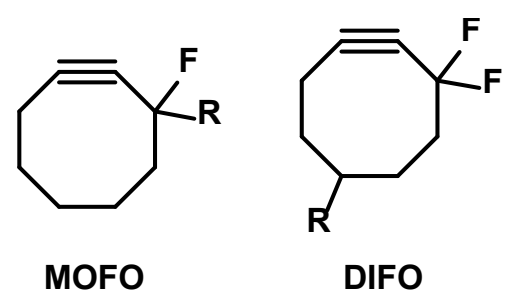

Figure 3.10: Structures of the activated cyclooctynes produced by the Bertozzi lab, a monofluoromethylene cyclooctyne (MOFO), and a difluoromethylene cyclooctyne (DIFO).

The Boons lab developed alternative activated cyclooctynes, in this case activated through the increased ring strain introduced via two fused benzo groups, as in Figure 3.11. ${ }^{147}$ It was expected that 4-dibenzocyclooctynols should have enhanced reactivity relative to cyclooctynes as a result of the increased ring strain from the aromatic groups as well as the additional conjugation providing electron density to the alkyne. ${ }^{147}$ The conjugated system in this case would be protected from nucleophilic attack at the alkyne 
due to steric hindrance from the ortho hydrogen. The dibenzocyclooctyne reagent (DIBO) has reaction rates equivalent to the previously outlined DIFO reagent from the Bertozzi lab. ${ }^{147}$

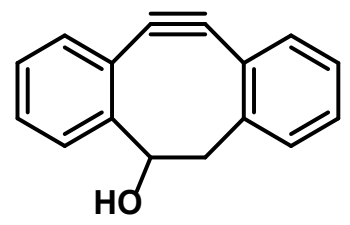

Figure 3.11: Structure of the strained alkyne dibenzocyclooctyne (DIBO), produced by the Boons lab. In 2010, the Bertozzi lab again sought to improve on the already demonstrated DIBO and DIFO systems by designing a probe with further enhanced reactivity. With the goal of balancing reactivity and stability of the cyclooctyne, it was demonstrated that the incorporation of an amide into the cyclooctyne ring would allow for the generation of a pseudo ene-yne system with enhanced reactivity. ${ }^{148}$ The incorporation of an amide would also enhance its water solubility and hence pharmacokinetic applicability. This lead to the development of the novel cyclooctyne biarylazacyclooctyne (BARAC) as shown in Figure 3.12, with a 12-fold higher rate of reaction than DIFO, and over 450 times higher rate of reaction than an unactivated cyclooctyne while still maintaining the chemoselectivity found in azide-alkyne cycloaddition reactions. ${ }^{148}$ Enhanced reactivity would allow for greater sensitivity in readouts when BARAC is tagged with a fluorophore, as well as a more rapid readout. 


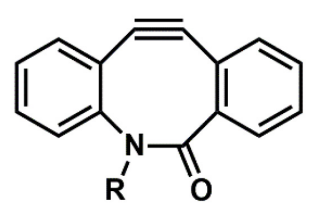

Figure 3.12: Structure of the biarylazacyclooctyne (BARAC) strained alkyne produced by the Bertozzi lab.

\subsubsection{Example of click reactions used in aptamer sensor development}

An extensive literature survey has shown that there is only one publication on the topic of analyte detection through an azide-alkyne cycloaddition using an aptamer as the recognition element. In the work of Sharma and Heemstra in 2011, the binding event of a split aptamer was used to bring a cyclooctyne and an azide tag into sufficient proximity to allow a strain promoted azide-alkyne cycloaddition. ${ }^{149}$ This was used to develop an aptamer sensor for cocaine, such that the binding of the cocaine molecule by the two aptamer halves results in the reactive moieties being close enough to undergo a cyclization resulting in strand ligation as shown in Figure 3.13. Gel-electrophoresis could then be used to quantify the amount of ligation between the two sequences to produce a new longer oligonucleotide strand. 
A

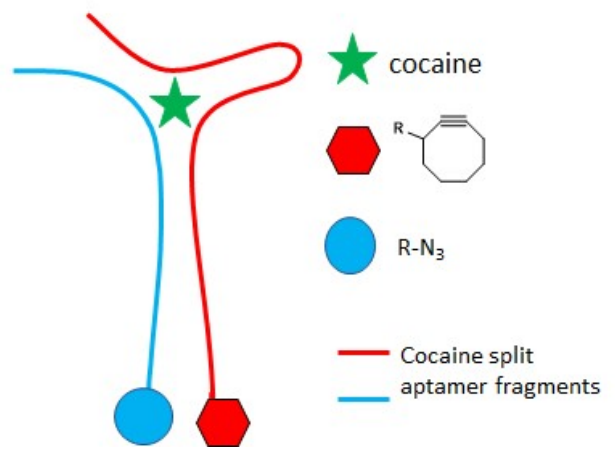

B

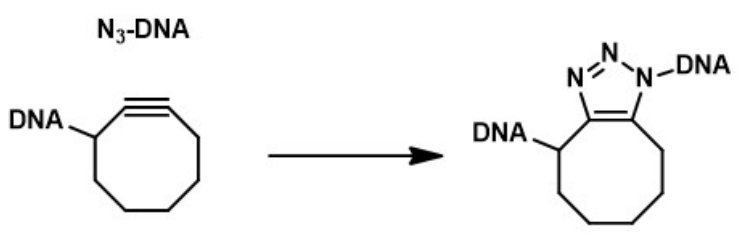

Figure 3.13: A) Depiction of the cocaine sensor produced by the Heemstra research group, consisting of two labeled cocaine split aptamers brought together by target binding to align the cylooctyne and azide DNA modifiers to permit the strain promoted cycloaddition. B) The strain-promoted azidealkyne cycloaddition to ligate the two DNA sequences shown in $A$ to generate a triazole. The disadvantage of this system is that gel electrophoresis is not a convenient way to monitor the presence of an analyte, and therefore alternative detection strategies needed to be developed.

\subsubsection{Fluorogenic click chemistry}

In many applications, the goal of a click reaction is to label a molecule so that it can be readily detected using some form of analytical device. One of the most common methods of labelling used in bioanalytical applications is the use of a fluorescent tag; this can be accomplished using click chemistry through the attachment of a fluorescent dye (azide or alkyne modified) to the click target. ${ }^{150}$ An alternative would be to develop fluorogenic click probes, such that upon successful azide-alkyne cycloaddition, a fluorophore is generated from the previously non-emissive structure. The first example of a fluorogenic click reaction was developed by Zhou and Fahrni in 2004 and was based on the electron-donating properties of triazole rings altering the fluorescence emission spectrum of a conjugated coumarin. ${ }^{151}$ It had previously been demonstrated by de Melo et al. that the photophysical behaviour of coumarins was dependent on the substitution 
profile, with substitutions in the 7-position of the coumarin having the greatest effect. ${ }^{152}$ The fluorescent quantum yield of coumarins is dependent on the relative order of the energy levels for its two lowest excited states. ${ }^{150-151}$ It was demonstrated that the reaction of 7-alkynecoumarin and an azide as shown in Figure 3.14 results in the formation of an electron donating triazole, which has a 20 -fold higher quantum yield with an absorption maximum of $328 \mathrm{~nm}$ and emission maximum of $415 \mathrm{~nm} .^{151}$

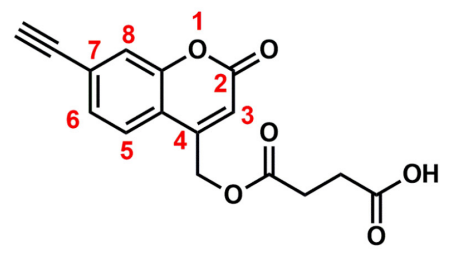<smiles>N#CCc1ccc(C(=O)O)cc1</smiles>
$\mathrm{CuSO}_{4}$, ascorbic acid<smiles>O=C(O)CCC(=O)OCc1cc(=O)oc2cc(-c3cn(Cc4ccc(C(=O)O)cc4)nn3)ccc12</smiles>

Figure 3.14: Scheme of the fluorogenic click reaction developed by Zhou and Fahrini, involving the copper catalyzed azide-alkyne cycloaddition between an alkyne coumarin and benzyl azide. Coumarin numbering convention is shown in red on the structure on the right.

Sivakumar et al. demonstrated that a similar system could be developed for azide substitutions at the 3-position on coumarin. ${ }^{153}$ As with 7-position substitution, a dramatic increase in quantum yield is observed upon formation of the triazole as a result of decreased electron density.
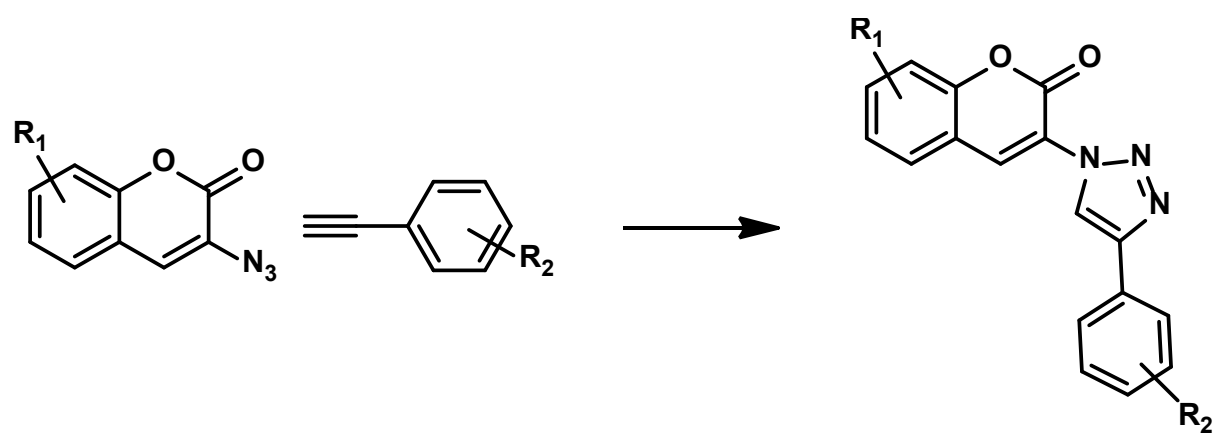

Figure 3.15: Scheme of the fluorogenic click reaction developed by Sivakumar et al. for the reaction between a 3-azido substitued coumarin and phenyl azide. 
Wong et al. designed a system using 1,8-naphthalimide, whose fluorescent properties can be altered by substitution at the 4-position. ${ }^{154}$ An alkyne or azide substituted variant of the naphthalimides was produced as in Figure 3.16, and reacted with either an azido or an alkyne counterpart resulting in the formation of a triazole, which is highly fluorescent.<smiles>C#Cc1ccc2c3c(cccc13)C(=O)N(CC)C2=O</smiles>

non-fluorescent

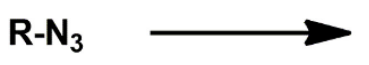<smiles></smiles>

Figure 3.16: Scheme of the fluorogenic click reaction developed by Wong et al., in which an alkyne modified naphthalimide is reacted with an azide to generate a fluorophore from a profluorophore.

Xie and Wang derivatized boron-dipyrromethene (BODIPY) dyes with an azide group at the 3-position on the pyrrole as shown in Figure 3.17. ${ }^{150}$ BODIPY dyes have high quantum yields regardless of solvent, but their photophysical properties can be easily altered by substituents at the 3 or 5 positions of the pyrrole ring. The azide modification quenches the fluorescence of the ring, and formation of the triazole through reaction with an alkyne restores fluorescence due to the weaker electron-donating characteristics of the triazole relative to an azide. 


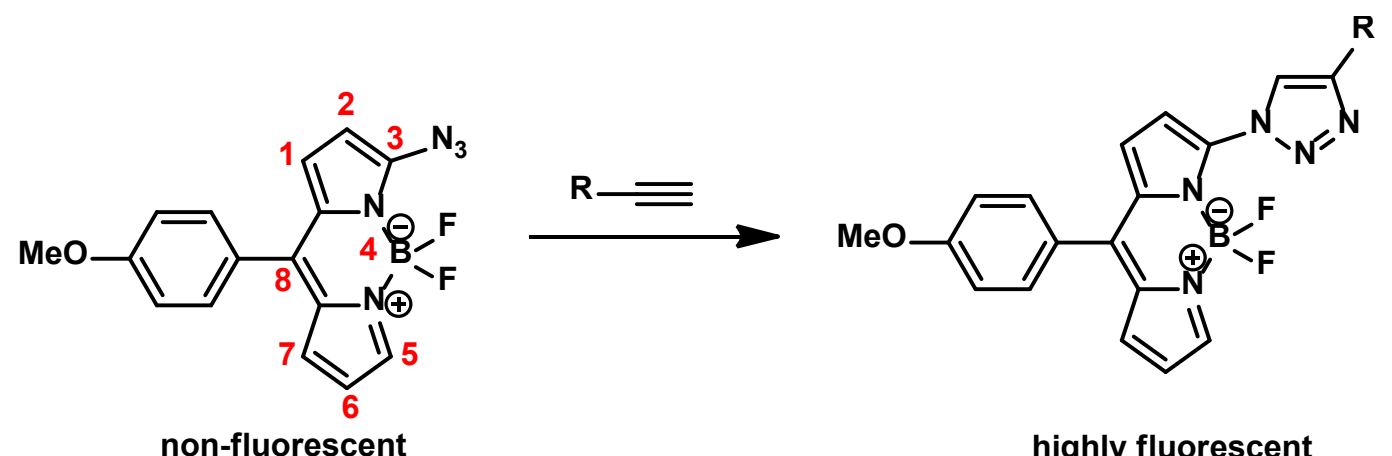

Figure 3.17: 3-azido modified boron-dipyrromethene (BODIPY) dyes act as proflorophores, activated to highly fluorescent molecules upon azide-alkyne cycloaddition with an alkyne. BODIPY numbering convention is indicated in red on the non-fluorescent structure.

Sun and Peng demonstrated the application of the fluorogenic azide-alkyne cycloaddition developed by Zhou and Farzini to templated DNA ligation reactions in 2013. ${ }^{151,155}$ By attaching a 7-alkynecoumarin to one sequence of DNA, and an azide tag to another sequence of DNA, a ligation reaction can be catalyzed in the presence of a sequence that is complementary to each, as shown in Figure 3.18. Upon ligation, a fluorescent triazole-coumarin is formed permitting its detection. ${ }^{155}$ In this study it was demonstrated that sequence anomalies in the complementary sequence to the two labeled oligonucleotide probes could be readily detected based on the fluorescence outcome of the reaction. A greater sequence mismatch results in a lower fluorescence intensity due to a lower yield of ligated product. 


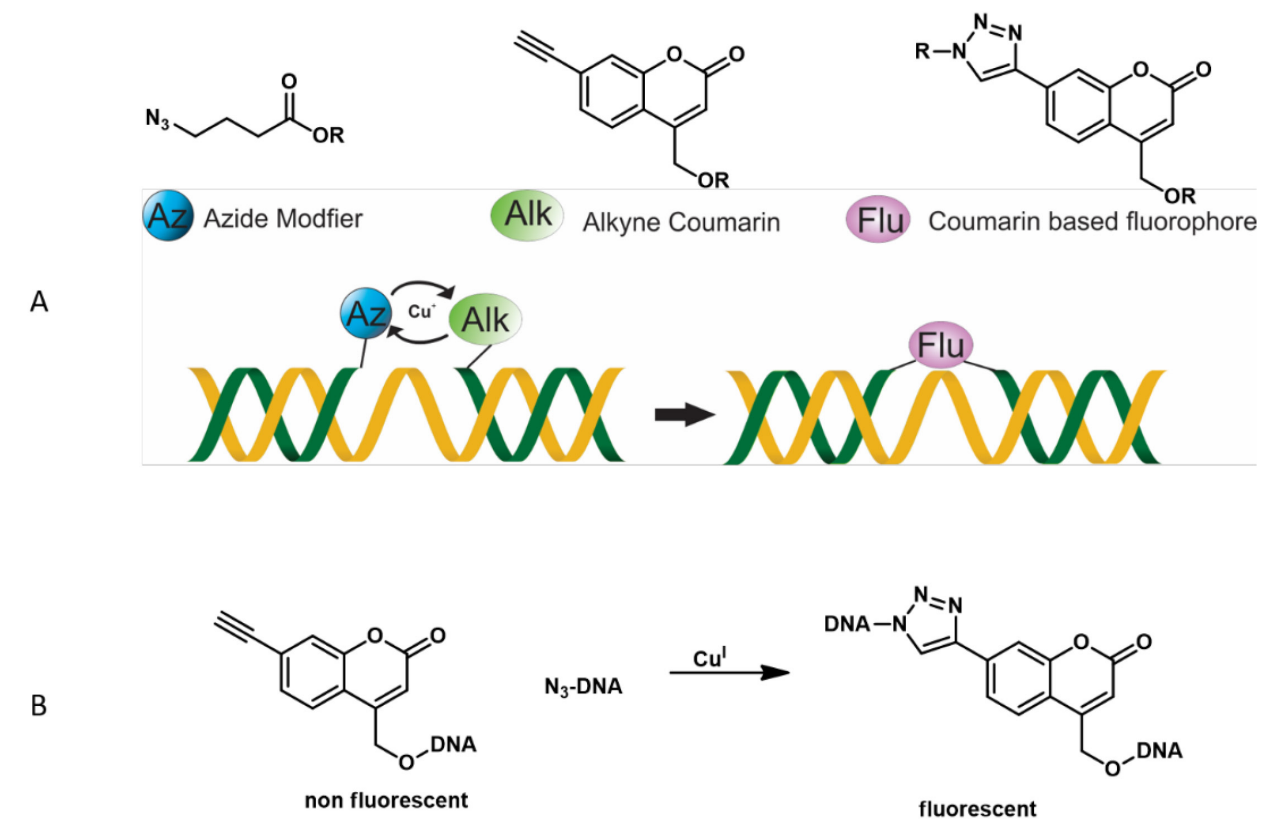

Figure 3.18: A) Sensor design of Sun and Peng, based on the detection of a target sequence (yellow) through a fluorogenic copper catalyzed azide-alkyne cycloaddition between an alkyne-coumarin and an azide. B) The fluorogenic reaction between the 7-alkyne coumarin profluorophore labeled DNA and the azide labeled DNA to generate the fluorescent triazole.

\subsubsection{The application of fluorogenic click modifiers to DNA aptasensors}

The limitation of the quenched autoligation probes explored in chapter 2 was the lack of stability of the electrophilic quencher and its non-specific loss leading to challenges associated with a high background fluorescence. Once an azide or an alkyne is incorporated into a biomolecule, they are considered biorthogonal chemical modifiers, as there are few extraneous reactions that they will undergo in complex environments. ${ }^{144,156-}$ 157 This makes click chemistry relevant in sensor development, as side reactions between the chemical modifiers and any foreign molecules are virtually non-existent when compared to the rapid cycloaddition that occurs between the azide and alkyne in the 
presence of copper(I).

To expand the scope of click chemistry to this newly developed field of controllable chemical reactions using DNA aptamers, DNA modifiers like those produced by Sun and Peng would need to be synthesized. ${ }^{155}$ A synthetic method for the production of 7alkynecoumarin phosphoramidite designed by Sun and Peng would be undertaken and optimized to maximize the yield and stepwise purity. An overall synthesis proposal for the 7-alkynecoumarin DNA phosphoramidite is shown in Figure 3.19. The initial synthetic strategy was to brominate ethyl acetoacetate and then react it with resorcinol via a Pechmann condensation to produce a brominated coumarin. Bromide hydrolysis, followed by triflate activation and a Sonogoshira reaction would produce the coumarin modified at the 7-position with an alkyne following deprotection. This alkyne coumarin could then be diverted in two different directions depending on the project at hand. In one strategy, it was converted into a phosphoramidite as shown in Figure 3.19 for direct incorporation into DNA on the DNA synthesizer. In the second strategy, which is not shown, the alkyne coumarin can be converted into an activated ester for reaction with an amine modifier on already synthesized DNA. 


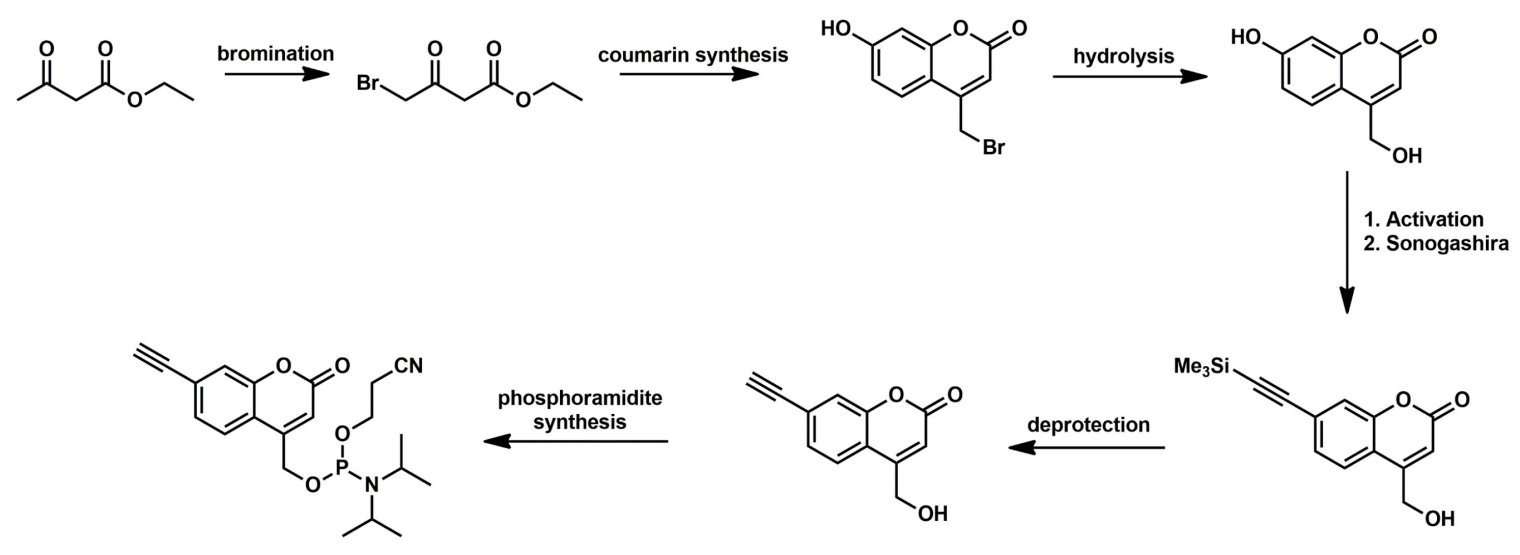

Figure 3.19: Overall synthesis scheme for the production of the 7-alkyne coumarin phosphoramidite starting from ethyl acetoacetate, as proposed by Sun and Peng. ${ }^{155}$

The azide modifier would be incorporated onto DNA as an NHS ( $N$ hydroxysuccinimide) activated ester of 4-azidobutanoic acid as shown in Figure 3.20. Using the method originally reported by Sun and Peng, it was proposed that starting from methyl-4-bromobutanoate an azidification reaction could be used to install the azide followed by hydrolysis to deprotect the carboxylic acid and produce 4-azidobutanoic acid. Reaction with NHS would permit synthesis of the NHS-activated ester of 4-azidobutanoic acid, which could then be readily coupled to DNA through an amine modifier on the DNA.

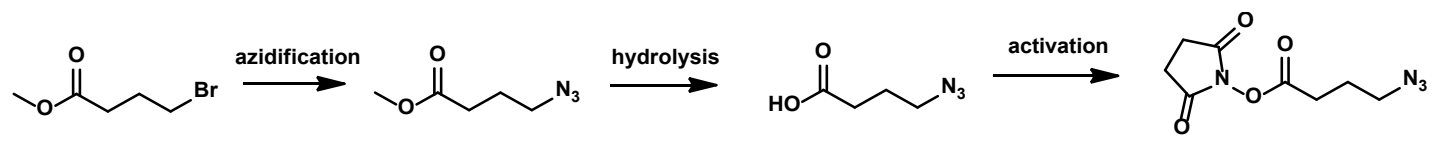

Figure 3.20: Overall synthesis scheme for the production of the NHS-activated ester of 4-azidobutanoic acid proposed by Sun and Peng. ${ }^{155}$

Once these two modifiers are synthesized, the end goal was to directly incorporate them into DNA in a tripartite system, as had been shown in the QUAL sensor system in chapter 2. Much like the work of Sun and Peng, the goal was to initially demonstrate that 
a fluorogenic ligation between two probes labeled with the alkyne-coumarin and the azide could be enabled through the presence of a complementary sequence, in this case a DNA aptamer. The DNA aptamer would allow this fluorogenic azide-alkyne cylcoaddition to be used as a sensor by reducing the ability of these complementary sequences to bind to the DNA aptamer once it undergoes a target binding event. A reduction in binding would result in a decrease in the proximity-based catalysis enabled by the presence of the DNA aptamer, thereby permitting measurement of the amount of target based on the extent of the click reaction. Later, this would be expanded to a single aptamer system so that aptamer shape change would result in the proximity-based catalysis, as well as a split aptamer system where a divided aptamer undergoing a target binding event would permit proximity-based catalysis.

\subsection{Results and discussion}

\subsubsection{Pechmann condensation}

In order to synthesize the alkyne-coumarin phosphoramidite, the work of Sun and Peng was used as the starting point, with their overall synthetic scheme depicted in simplified form in Figure 3.19. ${ }^{155}$ On examination of their overall synthesis workflow, it was observed that many of the early steps in the scheme suffered from low yields, with yields of the bromination step and coumarin synthesis steps being particularly ominous at $65 \%$ and $20 \%$ respectively. A side goal was to improve the overall yield of the synthetic scheme using alternative methods. The percent yield of the synthetic plan described by Sun and Peng was $4.5 \%$ from ethylacetoacetate to the alkyne-coumarin phosphoramidite. 
It was therefore decided that optimization of the synthetic scheme was the key, and strategies involving cutting out entire steps through commercial availability of starting materials and/or improving the yield of a given step through alternative reagents were explored.

In the first two steps of the synthetic plan of Sun and Peng, the coumarin core was synthesized by first brominating ethyl acetoacetate to form ethyl 4-bromoacetoacetate, which was then reacted with resorcinol in a Pechmann condensation to form a coumarin bearing a methylene bromide handle at the 4-position and an alcohol at the 7-position. Ethyl-4-bromoacetoacetate is not commercially available, but the related ethyl-4chloroacetoacetate could be purchased from Sigma Aldrich, thereby eliminating one entire step with its reported yield of $65 \%$. The subsequent step in the synthesis of Sun and Peng was the Pechmann condensation, which is generally defined as the synthesis of coumarins through the reaction of phenols with $\beta$-keto esters. ${ }^{158-159}$ The reported yield for the Pechmann condensation in sulfuric acid was $20 \%$, and alternative chemistry was explored. The first strategy taken from literature for the synthesis of the coumarin core $\mathbf{3 . 2}$ from ethyl-4-chloroacetoacetate $\mathbf{3 . 1}$ used toluene with $p$-toluenesulfonic acid and a short reaction time of 45 minutes with heating to reflux at a reported yield of $70 \%$ (as demonstrated by Fan et al.). ${ }^{160}$ This reaction was attempted twice with no recovered product on either occasion, instead forming a brown polymer sludge that did not resemble the expected white solid product in form or ${ }^{1} \mathrm{H}$ NMR analysis. Recrystallization of resorcinol from toluene was also performed for the second reaction to ensure the resorcinol was of the highest purity, but this did not change the result of the reaction. The byproduct 
could not be identified, and in both cases, it was assumed that heating of the mixture was resulting in some form of unexpected polymerization pathway. Therefore, alternative lowtemperature synthesis strategies for the coumarin core 3.2 were explored in literature.

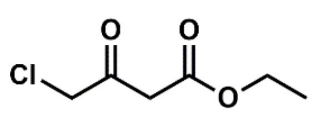

3.1

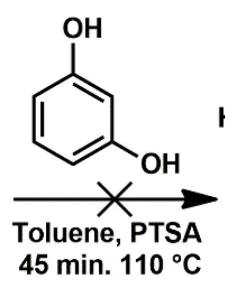

$45 \min .110^{\circ} \mathrm{C}$

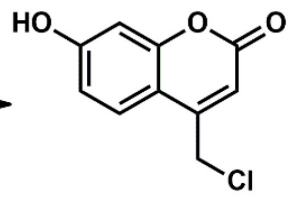

3.2

Figure 3.21: The reaction of ethyl-4-chloroacetoacetate with resorcinol in refluxing toluene using PTSA as reported by Fan et al. proved unsuccessful with no recoverable product. ${ }^{160}$

In a synthetic method proposed by Chen et al., the coumarin core $\mathbf{3 . 2}$ could be synthesized from resorcinol and ethyl-4-chloroacetoacetate in a marginally longer reaction at low temperatures but using concentrated sulfuric acid as the solvent as shown in Figure 3.22. ${ }^{161}$ This reaction was also shown to produce similar yield to that reported in the method of Fan et al (61.8\%), but had the advantage of not requiring the use of column chromatography to purify the final compound, instead proposing the use of recrystallization from ethanol to purify 3.2. Running the reaction under these proposed conditions in an initial test resulted in a yield of $30 \%$, which was improved to $71 \%$ through recrystallization of the resorcinol from toluene before its use. The product was confirmed through ${ }^{1} \mathrm{H}$ NMR analysis, which showed a strong agreement with characterization reported in the literature and was used in the ensuing steps without further analysis or purification. 
<smiles>CCOC(=O)CC(=O)CCl</smiles>

3.1

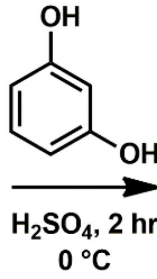

$71 \%$<smiles>O=c1cc(CCl)c2ccc(O)cc2o1</smiles>

3.2

Figure 3.22: The reaction of ethyl-4-chloroacetoacetate with resorcinol in the presence of sulfuric acid could be run at $0^{\circ} \mathrm{C}$, and resulted in a yield of $71 \%$

The synthesis of $\mathbf{3 . 2}$ was significantly improved from literature through the exploration of alternative strategies and different reaction solvents. In the work of Sun and Peng, a two-step yield of $13 \%$ to produce 3.2 from ethyl 4-bromoacetoacetate was attained, which is significantly lower than the $71 \%$ yield reported herein. A table of reaction conditions and resulting yield is shown in Table 3.1, including the initial strategies and the yield of repeated trials using the sulfuric acid solvent system. Interestingly, since this synthetic method was used in this project in early 2015 a variety of publications on the synthesis of 3.2 using these same conditions have been reported, with yields ranging from 65 to $75 \%$ as seen here. ${ }^{162-165}$

Table 3.1: Reaction conditions and repeated trials for the product of 3.2 with yield indicated.

\begin{tabular}{|c|c|c|}
\hline Conditions & Result & Yield $(\%)$ \\
\hline PTSA, refluxing toluene & $\begin{array}{l}\text { No product- brown } \\
\text { polymer }\end{array}$ & 0 \\
\hline Sulfuric acid, $0^{\circ} \mathrm{C}$ & Successful isolation of $\mathbf{3 . 2}$ & 31 \\
\hline Sulfuric acid, $0^{\circ} \mathrm{C}$, recrystallized resorcinol & Successful isolation of $\mathbf{3 . 2}$ & 71 \\
\hline Sulfuric acid, $0^{\circ} \mathrm{C}$, recrystallized resorcinol & $\begin{array}{c}\text { Successful isolation of } \mathbf{3 . 2} \\
\text { of lower purity }\end{array}$ & 68 \\
\hline
\end{tabular}




\subsubsection{Coumarin hydrolysis}

The ensuing reaction in the production of the alkyne-coumarin phosphoramidite is the hydrolysis of the methylene chloride at the 4-position of the coumarin to produce an allylic alcohol. This allylic alcohol will become the synthetic handle for the incorporation of the phosphoramidite functionality at the end of the synthesis. Using the procedure outlined by Sun and Peng, the chlorocoumarin 3.2 was dissolved in water and heated to reflux over eight hours, with product recovered as crystals from the cold solvent as shown in Figure 3.23. ${ }^{155}$ This reaction was quite efficient, with yields of 79 and $80 \%$ of $\mathbf{3 . 3}$ observed on individual trials, which is slightly lower than the $90 \%$ yield reported by Sun and Peng, but is similar to reported yields from many examples of this reaction in literature. ${ }^{155}$ The product was analyzed by ${ }^{1} \mathrm{H}$ NMR, which corresponded well with the literature, except for a large remaining water peak. To avoid issues with the presence of water in the subsequent triflation step, the crystalline product was lyophilized overnight before proceeding to the next step.

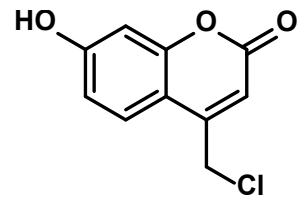

3.2

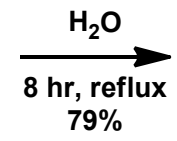

$79 \%$

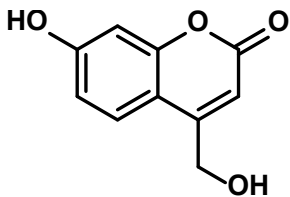

3.3

Figure 3.23: The hydrolysis of alkyne coumarin 3.2 in water to produce hydroxycoumarin 3.3.

\subsubsection{Coumarin triflation}

The next synthetic step in the pathway sought to install a triflate on the phenol hydroxyl group found at the 7-position of the coumarin to start the pathway towards incorporation of the alkyne modifier. By activating the phenol at position 7 with a triflate, 
the substrate is prepared for a subsequent Sonogashira reaction to install the alkyne modifier. Initially, the protocol of Sun and Shen was adopted for the synthesis, but upon further exploration of their protocol, it was determined that it was not possible to attain the reported $74 \%$ yield as only 0.3 equivalents of $N$-phenyl-bis(trifluoromethanesulfonimide) relative to 3.3 were used, which would result in a maximum theoretical yield of $30 \%$. Upon further exploration, it was determined that the procedure had been derived from another source without updating the ratios of reagents, and therefore an alternative protocol needed to be found. ${ }^{151}$ In the original work of Zhou and Fahrni for the development of the 7-alkyne coumarin reagents, a protocol for the synthesis of 3.4 was reported with a yield of $83 \%$ from 3.3 as shown in Figure 3.24. ${ }^{151}$ In this protocol, 1.1 equivalents of of $N$-phenylbis(trifluoromethanesulfonimide) relative to $\mathbf{3 . 3}$ were used, making the yield more plausible. Post-synthesis, the triflated coumarin was purified using dry-column vacuum chromatography (DCVC), a protocol developed by Pedersen and Rosenbhom for the rapid purification of large samples as a simplified alternative to a traditional flash chromatography setup. ${ }^{166}$ In this protocol, a short silica adsorbent pad of 6-7 $\mathrm{cm}$ is packed into a sintered glass funnel, which is attached to a separatory funnel. After packing and loading of the sample adsorbed on Celite, the mobile phase is run through the column one fraction at a time and collected in the separatory funnel. This protocol allows for rapid purification of large samples without the need for dividing it into smaller samples as would be required for flash chromatography, and also provides high resolving power - similar to that seen in TLC purification procedures. ${ }^{166}$ After purification using a constant $15 \%$ ethyl acetate/hexane mobile phase, $94 \%$ yield of 3.4 was attained, with a purity by NMR of 
$90.9 \%$ (85\% adjusted yield) - the only contaminant being residual DIPEA, which was deemed inconsequential for the subsequent reaction. Comparison of the obtained ${ }^{1} \mathrm{H}$ NMR spectrum to literature showed strong agreement, and therefore 3.4 was used without further purification and analysis.

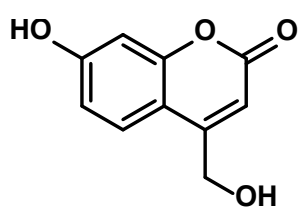

3.3

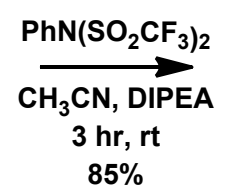

$85 \%$

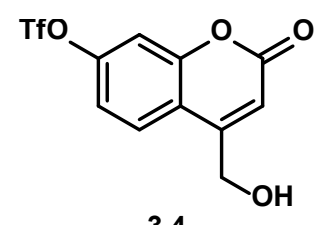

3.4

Figure 3.24: Triflation of the hydroxycoumarin 3.3 using the protocol of Zhou and Fahrni yielded the triflated coumarin 3.4 at $85 \%$ yield. ${ }^{151}$

\subsubsection{Sonogashira reaction}

The Sonogashira reaction is a reaction used for the coupling of terminal alkynes with aryl or vinyl halides or triflates in the presence of a palladium catalyst and typically a copper(I) cocatalyst. ${ }^{167-169}$ In this work, the Sonogashira reaction was performed on the activated triflate $\mathbf{3 . 4}$ to install a trimethylsilyl (TMS)-protected acetylene group at the 7position in the presence of a palladium catalyst and copper co-catalyst as shown in Figure 3.25. While operationally simple, the Sonogashira reaction for the installation of TMSacetylene proved quite capricious, with the reaction yield seemingly varying with no changes in the procedure and independently of starting material freshness. The reported synthesis of Sun and Peng produced a 90\% yield of $\mathbf{3 . 4}$ as a white solid, with yields from the attempts made in our lab ranging from $37 \%$ to $95 \%$ after DCVC purification as a green 
to white-brown to dark brown solid depending on the instance of the reaction as summarized in Table 3.2. In each trial of the Sonogashira reaction, the crude product came out as a black solid, suggesting the formation of palladium black and catalyst decomposition. The reaction was monitored by TLC, and it was determined that 30 minutes were required for the complete consumption of the triflate and minimal sideproduct formation. This timing coincided with the solution turning from a vibrant yellow to a dark green/black. It is likely that after this point the palladium catalyst has started to decompose, and therefore the reaction should be stopped earlier than the previously reported 3 hours. Though there were issues with the reaction and significant by-product formation that ended up streaking through to the final product, the reaction was deemed a success by NMR, through comparison of the ${ }^{1} \mathrm{H}$ NMR spectrum to literature. ${ }^{151,155}$

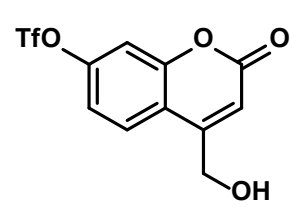

3.4

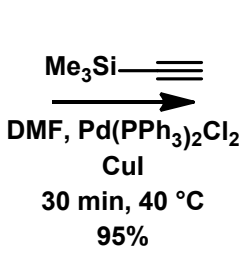

$95 \%$

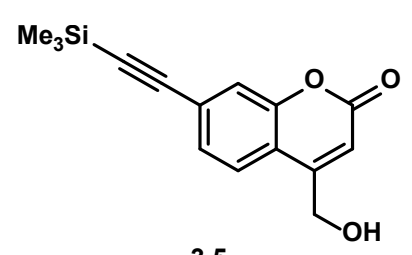

3.5

Figure 3.25: Sonogoshira reaction for the production of TMS-alkyne modified coumarin 3.5 from triflated coumarin 3.4 using the method of Sun and Peng. ${ }^{155}$ 
Table 3.2: Summary of the capricious nature of the Sonogoshira reaction as observed in this experiment. Yield appeared to vary regardless of reaction time, conditions, and freshness of the palladium catalyst.

\begin{tabular}{|c|c|c|c|c|}
\hline $\begin{array}{c}\text { Reaction } \\
\text { number }\end{array}$ & $\begin{array}{c}\text { Reaction } \\
\text { time }\end{array}$ & $\begin{array}{c}\text { State of Pd } \\
\text { catalyst }\end{array}$ & Product colour & Yield (\%) \\
\hline 1 & $3 \mathrm{hr}$ & $\begin{array}{l}\text { Fresh, yellow } \\
\text { catalyst }\end{array}$ & Light brown & 37 \\
\hline 2 & $30 \mathrm{~min}$ & $\begin{array}{l}\text { Opened once } \\
\text { before and } \\
\text { stored under } \\
\text { argon, yellow }\end{array}$ & Off-white & 95 \\
\hline 3 & $30 \mathrm{~min}$ & $\begin{array}{l}\text { Fresh, yellow } \\
\text { catalyst }\end{array}$ & Light brown & 91 \\
\hline 4 & $30 \mathrm{~min}$ & $\begin{array}{l}\text { Fresh, green- } \\
\text { yellow catalyst }\end{array}$ & Black & 41 \\
\hline 5 & $30 \mathrm{~min}$ & $\begin{array}{l}\text { Opened once } \\
\text { before and } \\
\text { stored under } \\
\text { argon, green- } \\
\text { yellow }\end{array}$ & Off-white & 85 \\
\hline
\end{tabular}

\subsubsection{Alkyne deprotection}

An alternative strategy that was explored was the deprotection of the TMS-alkyne in potassium carbonate/methanol mixtures. Jeon et al. demonstrated the deprotection of a similar 7-alkyne coumarin in potassium carbonate/methanol in 10 minutes at room temperature, noting that longer reaction times resulted in decomposition of the coumarin. ${ }^{170}$ Under the conditions reported in Figure 3.26, alkyne coumarin 3.6 was synthesized in a $90 \%$ yield in a clean reaction not requiring further purification. Significant water impurity remained in the product following extractions and washings, which was removed through azeotropic distillation with toluene. Purity was confirmed by comparison of the ${ }^{1} \mathrm{H}$ NMR 
to literature, which showed excellent agreement, allowing the coumarin to be used in the subsequent phosphoramidite synthesis step. ${ }^{151,155}$

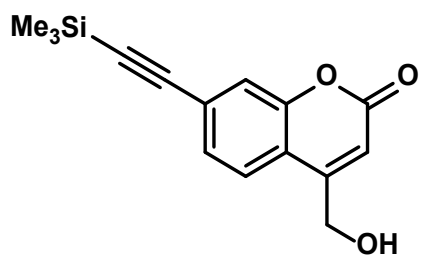

3.5

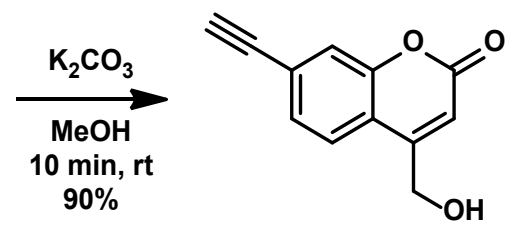

3.6

Figure 3.26: Deprotection of the TMS-alkyne 3.5 to produce the 7-alkyne-coumarin 3.6 using the protocol of Jeon et al. ${ }^{170}$

\subsubsection{Phosphoramidite synthesis}

To add the alkyne-coumarin to DNA, the traditional method for 5'-end labeling would be to convert it into a phosphoramidite. Sun and Peng proposed a method for the reaction of chloro-phosphoramidite with the alkyne-coumarin using DIPEA base, as shown in Figure 3.27. ${ }^{155}$ Significant care was taken in performing this reaction for several reasons. First, the chloro-phosphoramidite reagent is highly pyrophoric when in contact with oxygen and great care needs to be taken in its handling. Second, the alkyne-coumarin modifier had become quite time consuming and costly to prepare over its multi-stage synthesis, and therefore great care should be taken in producing the phosphoramidite efficiently to minimize waste. Finally, impurities need to be removed prior to adding the phosphoramidite to the DNA synthesizer, especially any impurity (especially water) that could act as a sacrificial nucleophile or electrophile, thereby decreasing the DNA yield through side reactions. For this reason, a synthetic method for the production of alkyne 
coumarin phosphoramidite was designed such that the alkyne-coumarin phosphoramidite was synthesized, purified, and incorporated onto the DNA in the same day. This synthetic procedure was also optimized by performing the reaction in a glove bag to protect against the pyrophoric nature of the chloro-phosphoramidite, while maximizing yields by ensuring an inert atmosphere throughout the reaction. Under such conditions, alkyne-coumarin phosphoramidite 3.7 could be synthesized and incorporated into DNA in a 14-hour day including purification on an Isolera automated purification column and 2 hours of lyophilization to remove any extraneous solvent or water before loading onto the DNA synthesizer. For this reason, and the limited amount of material, only a preliminary analysis of the synthetic outcome was performed by NMR. To confirm the purity of 3.7, the ${ }^{1} \mathrm{H}$ NMR spectrum was recorded and verified against literature following each synthesis immediately prior to lyophilization. Following DNA synthesis, the incorporation of the alkyne-coumarin phosphoramidite was confirmed through molecular-weight confirmation mass spectrometry (detailed in subsequent chapters). Synthesis yield of the phosphoramidite could potentially be improved through a more stringent purification of the starting material and more efficient washing, but this protocol permitted the rapid production of sufficient phosphoramidite for immediate consumption on the DNA synthesizer and was therefore maintained. 

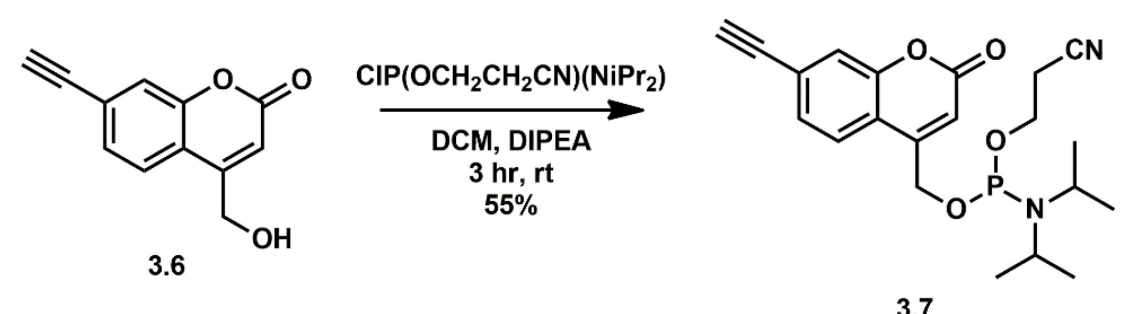

3.7

Figure 3.27: Production of the final alkyne-coumarin phosphoramidite 3.7 for immediate use on the automated DNA synthesizer.

\subsubsection{Alkyne-coumarin phosphoramidite synthesis overview}

The goal of this portion of the project was to synthesize the alkyne-coumarin and the alkyne-coumarin as precursors for subsequent reactions as they are not commercially available. As a side goal, improving the overall synthetic yield of the reaction pathway shown in Figure 3.28 to produce the alkyne-coumarin $\mathbf{3 . 6}$ and alkyne-coumarin phosphoramidite 3.7 was desired. Over the optimized five-step pathway to produce the alkyne-coumarin, and six steps to produce the alkyne-coumarin phosphoramidite an overall yield of $40.8 \%$ and $22.4 \%$ respectively was observed. In comparison, the synthetic pathway of Sun and Peng resulted in a percent yield of 5.8\% and $4.1 \%$ respectively, while that of Zhou and Fahrni reported 29\% yield for the alkyne coumarin in the same number of steps as our protocol. ${ }^{151,155}$ We can therefore state that we have improved the synthetic outcome of the reaction pathway in the process of producing the desired alkyne-coumarin 
and alkyne-coumarin phosphoramidite, demonstrating the value of reaction optimization in the field of bioconjugate chemistry - an aspect that is often overlooked.

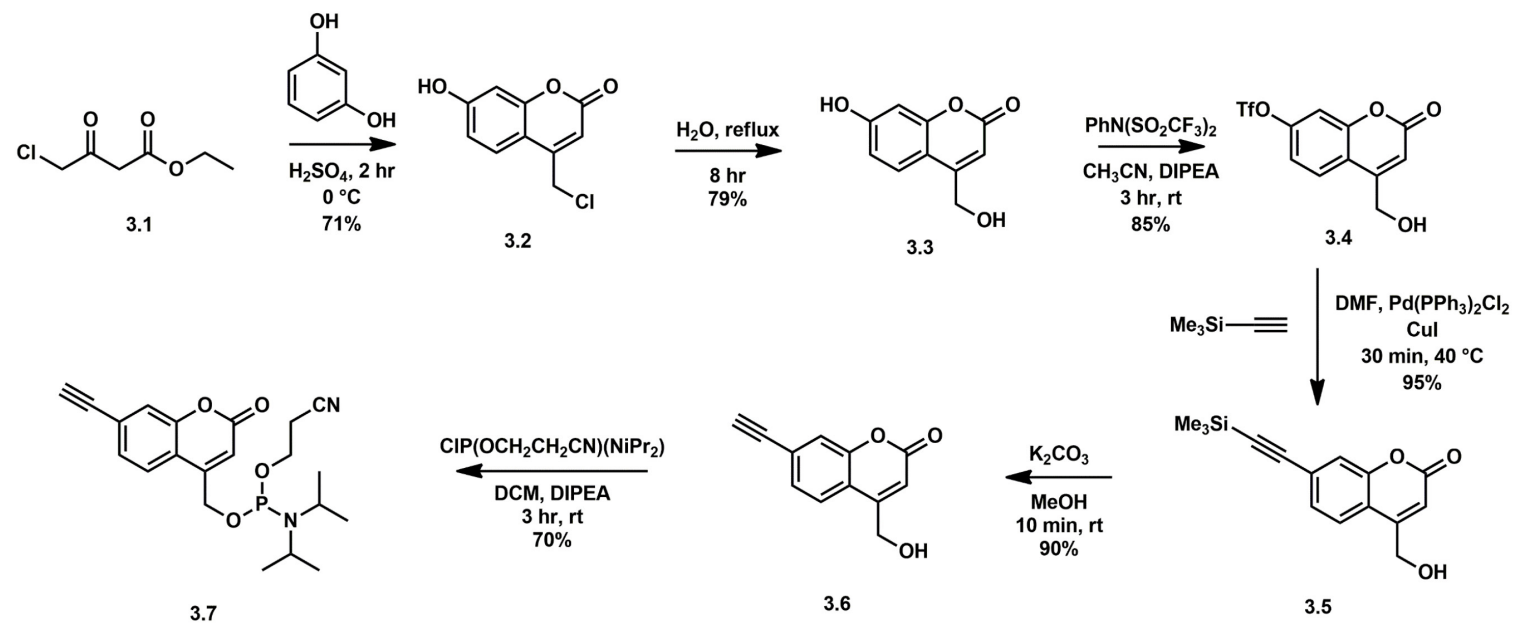

Figure 3.28: Overall synthetic pathway for the production of alkyne-coumarin 3.6 and alkynecoumarin phosphoramidite 3.7 with an overall yield of $40.8 \%$ and $22.4 \%$, respectively from ethyl-4chloroacetoacetate 3.1 .

\subsubsection{Synthesis of 4-azidobutanoic acid and commercial availability}

Having optimized a pathway to produce the alkyne-coumarin phosphoramidite, the same process had to be repeated for the azide modifier as shown in the proposed pathway (Figure 3.29). Again, synthetic challenges from the low yield in the method reported by Sun and Peng for the production of the azide modifier for DNA prompted the exploration of alternative strategies. One such strategy is shown in Figure 3.29, as adapted from DeForest and Anseth, starting from methyl 4-bromobutanoate 3.8. ${ }^{171}$ The proposed synthesis would be a continuous azidification followed by hydrolysis of the ethyl ester to 
produce 4-azidobutanoic acid $\mathbf{3 . 1 0}$ with no purification in between steps with an expected $92 \%$ yield and no purification required.<smiles>CCOC(=O)CCCBr</smiles>

3.8

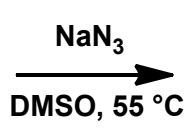

$9 \mathrm{hr}$<smiles>CCOC(=O)CCCN</smiles>

3.9

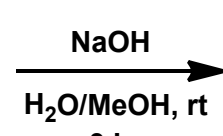

$3 \mathrm{hr}$

$92 \%$<smiles>N#CCCC(=O)O</smiles>

3.10

Figure 3.29: Optimized synthetic pathway proposed by DeForest and Anseth for the production of 4azidobutanoic acid from methyl-4-bromobutanoate. ${ }^{171}$

At the time of designing the project, the azide modifier was not commercially available, but in the time required to fully optimize the alkyne-coumarin phosphoramidite synthesis a commercially available material became available. Glen Research began to sell azidobutyrate NHS ester 3.11 (Figure 3.30) for applications in click chemistry, particularly in the attachment of clickable alkyne containing fluorescent dyes to DNA. Seeing as this chemistry was commercially available, the original plan to synthesize it in-house was discarded in favour of the commercially available material, and therefore the scheme reported in Figure 3.29 was not attempted. This NHS-activated ester was directly attached to amine modified DNA in subsequent chapters.

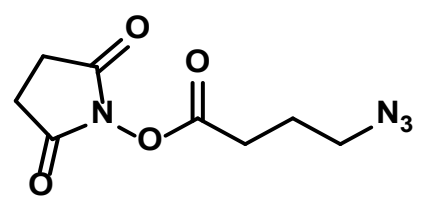

3.11

Figure 3.30: Structure of the commercially available azidobutryate NHS ester from Glen Research for the modification of DNA, thereby eliminating the need for an optimized synthetic procedure. 


\subsection{Experimental}

\section{General}

All reagents were purchased from commercial sources including Sigma Aldrich and Alfa Aesar and used as received unless otherwise noted below. Solvents including ACN, DMF, $\mathrm{MeOH}, \mathrm{DMSO}$, and DCM were purchased in their anhydrous variants from Sigma Aldrich with a Sure-Seal cap. Reactions were monitored by thin-layer chromatography (TLC) using glass-backed extra hard layer (60 A) TLC plates from Silicycle with UV-indicator, and visualized under UV light, iodine vapour, or using potassium permanganate stain. Flash chromatography was performed under either the dry column vacuum chromatography (DCVC) method with $6 \mathrm{~cm}$ columns detailed by Pedersen and Rosenbohm, ${ }^{166}$ or using the Biotage Isolera One automated chromatography system with its attached UV detector. Concentration in vacuo refers to rotary evaporation at $40{ }^{\circ} \mathrm{C}$ on a water bath with the appropriate vacuum pressure for the solvent in question. NMR spectra were recorded on a Bruker Avance III $300 \mathrm{MHz}$ spectrometer, and chemical shifts are recorded in parts per million. Tetramethylsilane (TMS) was used as an internal standard for deuterated chloroform $\left(\mathrm{CDCl}_{3}\right)$, while no internal standard was used with deuterated DMSO $\left(\mathrm{d}_{6}\right.$ DMSO) or deuterated acetone $\left(\left(\mathrm{D}_{3} \mathrm{C}\right)_{2} \mathrm{CO}\right)$. 


\section{4-(chloromethyl)-7-hydroxy-2H-chromen-2-one (3.2)}

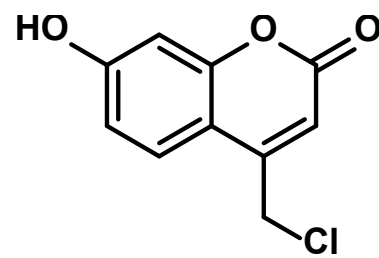

Resorcinol (10.01 g, $91 \mathrm{mmol}, 1.2$ equiv) that had been recrystallized from toluene was dissolved in $100 \mathrm{~mL}$ of concentrated sulfuric acid that had been cooled to $0{ }^{\circ} \mathrm{C}$ in a salt-ice bath. Complete dissolution of the resorcinol took approximately $1 \mathrm{hr}$, after which the solution became a light yellow. Ethyl 4-chloroacetoacetate (10 mL, $74 \mathrm{mmol}, 1.0$ equiv) was added dropwise over ten minutes to the stirring solution to produce a deep yellow colour. The reaction was stirred for $2 \mathrm{hr}$ while maintaining the temperature at a constant 0 ${ }^{\circ} \mathrm{C}$, and then worked up by pouring the reaction mixture onto $1 \mathrm{~L}$ of ice-water while stirring. Immediately upon addition to the ice bath, an off-white precipitate was formed and filtered off and washed three times with $50 \mathrm{~mL}$ deionized water. Upon standing, the off-white precipitate slowly turned pink, and was recrystallized from ethanol to yield a pure white solid of $11.1 \mathrm{~g}$ (71\% yield, $98.5 \%$ purity by NMR). Product showed agreement with expected literature ${ }^{1} \mathrm{H}$ NMR spectra with a small ethanol impurity. ${ }^{161}$

${ }^{1}$ H NMR $\left(300 \mathrm{MHz}\right.$, Acetone- $\left.d_{6}\right) \delta 9.51(\mathrm{~s}, 1 \mathrm{H}), 7.72(\mathrm{~d}, J=8.8 \mathrm{~Hz}, 1 \mathrm{H}), 6.90(\mathrm{dd}, J=$ 8.7, $2.4 \mathrm{~Hz}, 1 \mathrm{H}), 6.79(\mathrm{~d}, J=2.4 \mathrm{~Hz}, 1 \mathrm{H}), 6.40(\mathrm{~d}, J=0.9 \mathrm{~Hz}, 1 \mathrm{H}), 4.91(\mathrm{~d}, J=0.9 \mathrm{~Hz}$, $2 \mathrm{H})$. 
7-hydroxy-4-(hydroxymethyl)-2H-chromen-2-one (3.3)

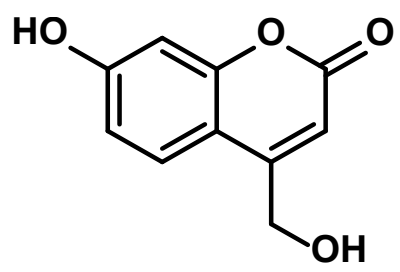

Chloro-coumarin 3.2 (10 g, $47 \mathrm{mmol}, 1$ equiv) was suspended in $1 \mathrm{~L}$ of deionized water and heated to reflux for eight hours. After 1 hour, an opaque gray suspension was formed. After 8 hours, the mixture was filtered while hot through Whatman No.2 paper and was cooled to room temperature. The filtered solution was extracted four times with $200 \mathrm{~mL}$ of EtOAc, and the combined organic layers were then washed once with brine, and dried over anhydrous $\mathrm{MgSO}_{4}$ and filtered, before concentrating under reduced pressure. The product was recovered as a white, sticky solid that was immediately transferred into a plastic Falcon tube and lyophilized overnight to remove any remaining water to yield 7.2 g (79\% yield) of white fluffy solid. The ${ }^{1} \mathrm{H}$ NMR spectrum showed excellent agreement with literature and was used without further purification or processing having accounted for the water in the spectrum being from the NMR solvent. ${ }^{161}$

${ }^{1}$ H NMR (300 MHz, DMSO-d $)_{6} \delta 10.52(\mathrm{~s}, 1 \mathrm{H}), 7.52(\mathrm{~d}, J=8.7 \mathrm{~Hz}, 1 \mathrm{H}), 6.77(\mathrm{dd}, J=$ 8.7, $2.4 \mathrm{~Hz}, 1 \mathrm{H}), 6.72(\mathrm{~d}, J=2.3 \mathrm{~Hz}, 1 \mathrm{H}), 6.23(\mathrm{t}, J=3.0 \mathrm{~Hz}, 1 \mathrm{H}), 5.61-5.55(\mathrm{~m}, 1 \mathrm{H})$, $4.70(\mathrm{~d}, J=4.1 \mathrm{~Hz}, 2 \mathrm{H})$. 


\section{4-(hydroxymethyl)-2-oxo-2H-chromen-7-yl trifluoromethanesulfonate (3.4)}

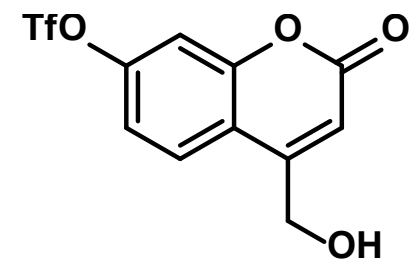

Hydroxycoumarin 3.2 (4.04 g, $21.0 \mathrm{mmol}$, 1.0 equiv) that had been azeotropically dried using toluene on a rotary evaporator was dissolved in $150 \mathrm{~mL}$ of dry acetonitrile in a flame dried flask under argon. $N$-phenyl-bis(trifluoromethanesulfonimide) $(8.27 \mathrm{~g}, 23.1 \mathrm{mmol}$, 1.1 equiv) was added to the solution along with DIPEA (4.82 $\mathrm{mL}, 27.7 \mathrm{mmol}, 1.2$ equiv). The reaction mixture was stirred for 3 hours at room temperature before diluting with 200 $\mathrm{mL}$ of EtOAc and washing three times with $150 \mathrm{~mL}$ of deionized water. The organic layer was dried over anhydrous $\mathrm{MgSO}_{4}$, filtered and concentrated in vacuo to yield $7.4 \mathrm{~g}$ of crude 3.3. DCVC purification ( $15 \%$ EtOAc/hexanes) yielded $6.4 \mathrm{~g}(93.9 \%$ yield, $90.9 \%$ by weight by NMR with DIPEA contaminant) of off-white solid 3.3. The ${ }^{1} \mathrm{H}$ NMR spectrum showed excellent agreement with literature with the remaining DIPEA contaminant deemed acceptable for the subsequent reaction. ${ }^{151,155}$

${ }^{1} \mathbf{H}$ NMR $\left(300 \mathrm{MHz}, \mathrm{CDCl}_{3}\right) \delta 7.65(\mathrm{~d}, J=8.8 \mathrm{~Hz}, 1 \mathrm{H}), 7.33(\mathrm{~d}, J=2.5 \mathrm{~Hz}, 1 \mathrm{H}), 7.25(\mathrm{dd}$, $J=8.8,2.5 \mathrm{~Hz}, 1 \mathrm{H}), 6.71(\mathrm{t}, J=1.6 \mathrm{~Hz}, 1 \mathrm{H}), 4.94(\mathrm{~d}, J=1.6 \mathrm{~Hz}, 2 \mathrm{H}), 2.06(\mathrm{~s}, 1 \mathrm{H})$. 


\section{4-(hydroxymethyl)-7-((trimethylsilyl)ethynyl)-2H-chromen-2-one (3.5)}

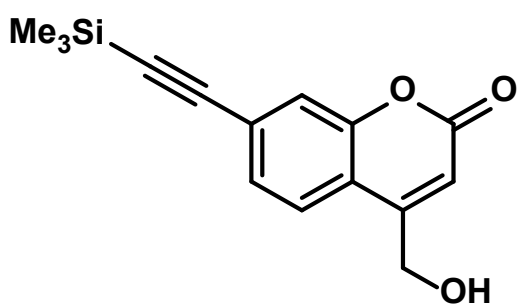

A mixture of 3.4 (2.50 g, $7.69 \mathrm{mmol}, 1.0$ equiv), dichloro(triphenylphosphine)-palladium (270 mg, $0.385 \mathrm{mmol}, 0.05$ equiv), CuI $(73.2 \mathrm{mg}, 0.385 \mathrm{mmol}, 0.05$ equiv), ethynyltrimethylsilane $(3.73 \mathrm{~mL}, 26.9 \mathrm{mmol}, 3.5$ equiv), triethylamine $(2.44 \mathrm{~mL}, 17.5$ mmol, 2.4 equiv), and DIPEA (2.03 mL, $11.7 \mathrm{mmol}, 1.6$ equiv) in dry DMF (100 mL) was flushed under argon for 20 minutes and stirred under inert atmosphere for 30 minutes. The mixture was diluted with EtOAc $(200 \mathrm{~mL})$, washed with water $(150 \mathrm{~mL}$ x 3), and brine $(150 \mathrm{~mL} \times 2)$. The organic layer was dried over anhydrous $\mathrm{Na}_{2} \mathrm{SO}_{4}$, filtered and concentrated in vacuo to yield $2.55 \mathrm{~g}$ of brown/black solid. DCVC purification (15\% ethyl acetate/hexanes then $30 \%$ ethyl acetate hexanes) yielded $1.99 \mathrm{~g} \mathrm{(95.1 \%} \mathrm{yield,} 86.7 \%$ purity by NMR) of light brown solid. The ${ }^{1} \mathrm{H}$ NMR spectrum showed excellent agreement with literature with the remaining DIPEA contaminant deemed acceptable for the subsequent reaction. ${ }^{151,155}$

${ }^{1} \mathbf{H}$ NMR $\left(300 \mathrm{MHz}, \mathrm{CDCl}_{3}\right) \delta 7.46-7.42(\mathrm{~m}, 2 \mathrm{H}), 7.35(\mathrm{dd}, J=8.4,1.4 \mathrm{~Hz}, 1 \mathrm{H}), 6.65(\mathrm{t}$, $J=1.6 \mathrm{~Hz}, 1 \mathrm{H}), 4.93(\mathrm{dd}, J=5.8,1.6 \mathrm{~Hz}, 2 \mathrm{H}), 0.29(\mathrm{~s}, 9 \mathrm{H})$. 
7-ethynyl-4-(hydroxymethyl)-2H-chromen-2-one (3.6)<smiles>C#Cc1ccc2c(CO)cc(=O)oc2c1</smiles>

Potassium carbonate (202 mg, $1.5 \mathrm{mmol}, 0.2$ equiv) was added to a solution of 3.5 (2.01 g, $7.3 \mathrm{mmol}, 1.0$ equiv) in methanol $(35 \mathrm{~mL})$. The mixture was stirred for 10 minutes at room temperature under argon. The reaction mixture was concentrated in vacuo and resuspended in ethyl acetate $(50 \mathrm{~mL})$, before washing with water $(20 \mathrm{~mL} \mathrm{x3})$, brine $(20 \mathrm{~mL})$, and drying over anhydrous $\mathrm{Na}_{2} \mathrm{SO}_{4}$ to yield $1.32 \mathrm{~g}(90.1 \%$ yield $)$ of off-white product. The ${ }^{1} \mathrm{H}$ NMR spectrum showed agreement with literature apart from extraneous toluene, which was removed on a lyophilizer overnight, and was used in subsequent reactions without further purification or analysis. ${ }^{151,155}$

${ }^{1}$ H NMR $\left(300 \mathrm{MHz}\right.$, Acetone- $\left.d_{6}\right) \delta 7.72(\mathrm{~d}, J=8.1 \mathrm{~Hz}, 1 \mathrm{H}), 7.52-7.35(\mathrm{~m}, 2 \mathrm{H}), 6.58(\mathrm{t}$, $J=1.6 \mathrm{~Hz}, 1 \mathrm{H}), 4.93(\mathrm{~d}, J=1.7 \mathrm{~Hz}, 2 \mathrm{H}), 3.96(\mathrm{~s}, 1 \mathrm{H})$.

\section{2-cyanoethyl ((7-ethynyl-2-oxo-2H-chromen-4-yl)methyl) diisopropyl} phosphoramidite (3.7)<smiles>C#Cc1ccc2c(COP(OCCC#N)N(C(C)C)C(C)C)cc(=O)oc2c1</smiles> 
All reactions for the preparation of the phosphoramidite were performed in an argon purged glove bag. To a solution of $\mathbf{3 . 6}(40.0 \mathrm{mg}, 200 \mu \mathrm{mol}, 1.0$ equiv) in dichloromethane (4 mL) in a flame dried $20 \mathrm{~mL}$ vial, DIPEA $(63 \mu \mathrm{L}, 360 \mu \mathrm{mol}, 1.8$ equiv) and 2-cyanoethyl- $N, N$ diisopropylchlorophosphoramidite $(67 \mu \mathrm{L}, 300 \mu \mathrm{mol}, 1.5$ equiv) was added and the vial capped. The reaction mixture was homogenized on a vortex mixer at room temperature for three hours before diluting with ethyl acetate $(50 \mathrm{~mL})$ and removing from the glove bag. The organic layer was washed with water $(3 \times 40 \mathrm{~mL})$, and brine $(40 \mathrm{~mL})$ before concentrating in vacuo. The crude product was immediately resuspended in a minimum of toluene and loaded onto a Biotage Isolera 1 equipped with a Biotage SNAP KP-Sil $10 \mathrm{~g}$ column. Purification (EtOAC:hexane:triethylamine 66:33:1) yielded 3.7 as a white solid (44.0 mg, 55\% yield). The ${ }^{1} \mathrm{H}$ NMR spectrum showed agreement with literature apart from ethyl acetate contamination, and the product was lyophilized for 1 hour before diluting in acetonitrile for loading onto the BioAutomation 6 DNA synthesizer without further analysis. $^{155}$

${ }^{1}$ H NMR $\left(300 \mathrm{MHz}, \mathrm{CDCl}_{3}\right) \delta 7.46(\mathrm{~d}, J=9.0 \mathrm{~Hz}, 1 \mathrm{H}), 6.89-6.84(\mathrm{~m}, 2 \mathrm{H}), 6.45(\mathrm{t}, J=$ $3.0 \mathrm{~Hz} 1 \mathrm{H}), 4.96-4.79(\mathrm{~m}, 2 \mathrm{H}), 3.98-3.70(\mathrm{~m}, 4 \mathrm{H}), 2.69(\mathrm{t}, J=6.2 \mathrm{~Hz}, 2 \mathrm{H}), 1.24(\mathrm{~d}, J=$ $6.8 \mathrm{~Hz}, 12 \mathrm{H})$. 


\section{Chapter: Fluorogenic Click chemistry for the detection of thrombin}

\subsection{Statement of contributions}

All DNA synthesis and testing of the thrombin fluorogenic sensor was performed with the assistance of undergraduate students Jordan Scott and Spencer Boisjoli. Christopher Mattice performed all final experimentation, sensor evaluations, and calculations.

\subsection{Introduction}

\subsubsection{Thrombin}

Thrombin is a serine protease of significant research interest in both the biomedical community as well as the aptamer-based affinity assay community for different, but related reasons. In the biomedical community, interest in thrombin stems not only from its important role as the final enzyme in the blood coagulation cascade but also from its unique ability to cleave fibrinogen into the clot forming fibrin protein. ${ }^{172}$ Thrombin is implicated in both physiological and pathological coagulation, with implication in disease states such as Alzheimer's disease, cancer and various forms of thrombosis. ${ }^{173}$ Inefficient or impaired thrombin generation results in hemorrhaging, from the inability to form stable blood clots. ${ }^{172}$ The inability to regulate thrombin activity results in the blood clot forming in other locations separate from the damage, which is termed thrombosis. ${ }^{172}$ The importance of thrombin in the aptamer community will be further discussed in section 4.2.2.

Thrombin has a high degree of sequence homology with other serine proteases such as chymotrypsin, characterized by the presence of serine residues in the active site 
cleft and a similar proteolytic cleavage mechanism. ${ }^{172,174}$ Human $\alpha$-thrombin is 295 amino acids in length and can be further proteolyzed to form the 189 amino acid long $\beta$-thrombin and $\gamma$-thrombin, both of which have decreased cleavage activity. ${ }^{172,}{ }^{174} \quad$ Preferred substrates of the thrombin protease have a positively charged amino acid at the first position in the scissile bond. ${ }^{172}$ Structurally, thrombin contains two loops, 60 and $\gamma$, that frame the active site. The 60-loop is a hydrophobic loop lending structural rigidity to the active site while also interaction with the hydrophobic N-terminus of the substrate cleavage sequence. ${ }^{174}$ The $\gamma$-loop is more hydrophilic and will interact with the carboxy-terminus of the cleavage sequence. Thrombin also has two anion binding sites called exosite I and II, responsible for interactions with negatively charged cofactors and substrates. ${ }^{174}$ The sodium ion binding site in the structure of thrombin is of interest due to its ability to allosterically modulate the activity of thrombin. Binding of a sodium ion increases the protein's interaction with procoagulant factors over anticoagulant factors, and under physiological conditions, it is estimated that $60 \%$ of thrombin molecules have this site occupied. ${ }^{175}$

Under normal circumstances, thrombin is not present in the blood, rather existing as its inactive zymogen prothrombin at concentrations in the $1 \mu \mathrm{M}$ range. ${ }^{175}$ Once coagulation is initiated through vascular injury, the levels of thrombin in the blood can vary between $\mathrm{pM}$ to $\mu \mathrm{M}$ concentrations. ${ }^{173}$ Hemostasis is initiated in response to a vascular injury, through exposure of the cell surface receptor, tissue factor, which binds prothrombin and factor VIIA. ${ }^{175}$ FVIIA will then proteolytically activate another factor called FX, which will in turn activate prothrombin to thrombin. ${ }^{175}$ Thrombin generation in this step 
is quite inefficient, with some being used to activate other factors, namely FV and VIII. These activated factors will then enhance the function of FXa and IXa, proteinases involved in the sustained generation of thrombin. ${ }^{175}$ This constitutes a positive feedback loop for the enhanced production of thrombin from prothrombin.

The primary function of thrombin is the conversion of fibrinogen to fibrin through proteolytic cleavage. ${ }^{172}$ The initial cleavage triggers the formation of protofibrils from fibrin monomers, and then lateral aggregation of protofibrils to form networks. The extensive mesh network entraps aggregated platelets to form a blood clot that seals the site of vascular injury and limits the loss of blood. ${ }^{172,175}$ As mentioned previously, thrombin also interacts with a variety of pro-coagulant cofactors implicated in sustained blood coagulation and blood clot stabilization through cross-linking including factors V, VIII, XIII, and XI. ${ }^{175}$

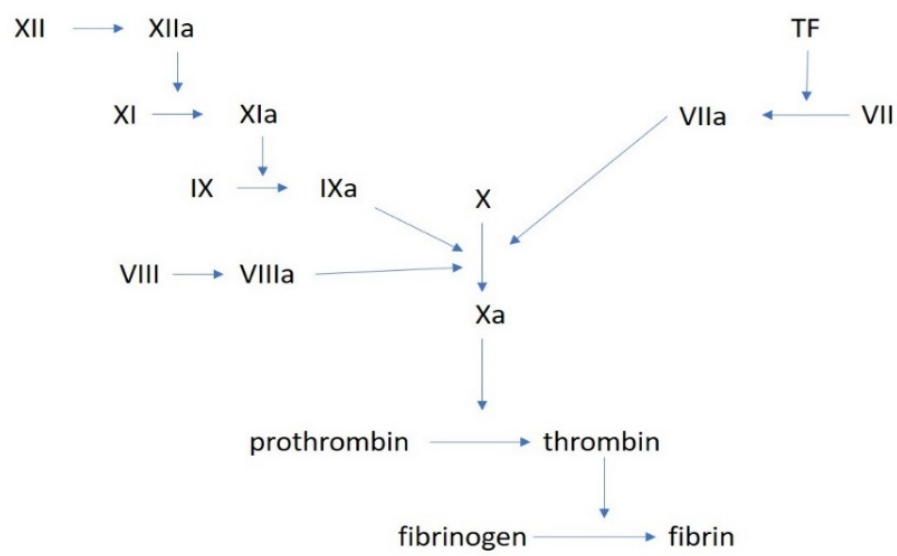

Figure 4.1: A simplified representation of the thrombin cascade for the conversion of prothrombin to its active thrombin form, which in turn cleaves fibrinogen to initiate formation of the fibrin clot.

Thrombin also has procoagulant activity, as was inferred in the discussion of the sodium ion binding sites. ${ }^{174}$ By binding to thrombomodulin, the substrate scope of 
thrombin is redirected from procoagulant to anticoagulant reactions. Proteins activated in this way include protein $\mathrm{C}$, which serves to downregulate thrombin production. ${ }^{175}$

Thrombin has a short half-life of $10-15 \mathrm{~s}$ in plasma through the action of antithrombin. By binding and cleaving the antithrombin protein, thrombin is locked in an irreversible inactive complex. ${ }^{175}$ This complex is cleared from the blood, and thrombin activity is reduced.

\subsubsection{Thrombin binding aptamer}

The first aptamer to human $\alpha$-thrombin was published by Bock et al. in $1992 .{ }^{176}$ After a five-cycle selection against thrombin with sequencing, a conserved sequence was observed in most of the clones from the 60-base random region in the starting library. This consensus 15-mer sequence (Table 4.1) maintained the same binding affinity as the parent full-length clones, with a measured dissociation constant of $100 \mathrm{nM}$. It was demonstrated that the thrombin binding aptamer elucidated could delay the formation of fibrin clots compared to a sample of thrombin with randomized or no DNA in solution, but to a lesser degree in plasma. ${ }^{177}$ It was postulated that the difference in potency was a result of nucleases or binding of prothrombin rather than activated thrombin. Binding of this 15mer aptamer sequence to thrombin was found to occur in the fibrinogen-recognition exosite. Another thrombin aptamer was elucidated by Tasset et al., this time as a 29-mer sequence with a higher affinity for thrombin and the ability to bind at the heparin binding exosite. ${ }^{178}$ This $29-$ mer sequence has a dissociation constant of $0.5 \mathrm{nM}$, and maintains a similar consensus sequence to that found in the 15-mer aptamer (Table 4.1). 
Table 4.1: Sequence of thrombin binding aptamers developed by Bock and Tasset.

\begin{tabular}{c|c} 
& Aptamer sequence \\
\hline Bock thrombin aptamer $^{177}$ & GGTTGGTGTGGTTGG \\
Tasset thrombin aptamer $^{178}$ & AGTCCGTGGTAGGGCAGGTTGGGGTGACT
\end{tabular}

Both short aptamer sequences can form a G-quadruplex structure as shown in Figure 4.2, with this switch occurring upon binding of human $\alpha$-thrombin. ${ }^{173} \mathrm{~A} \mathrm{G}$ quadruplex is a non-canonical nucleic acid conformation formed by guanine rich oligonucleotides, with the central structure being the formation of a guanine tetrad. ${ }^{179} \mathrm{~A}$ guanine tetrad consists of a planar arrangement of four guanine nucleobases interacting through their Hoogsteen faces. Stacking of two or more of these guanine tetrads results in the formation of a G-quadruplex, which are also further stabilized by a metal cation, such as $\mathrm{K}^{+}$, located in the central cavity of the quadruplex. ${ }^{173,180}$ Structural studies have demonstrated that in solution the thrombin binding aptamer forms a unimolecular antiparallel quadruplex with a chair like conformation, but it has also been demonstrated to form biomolecular and parallel quadruplexes dependent on aptamer concentration. ${ }^{173}$, 179-180 This corresponds well with the most commonly observed G-quadruplexes in aptamers. 
A

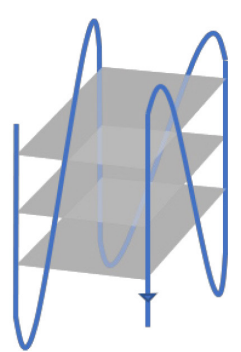

B

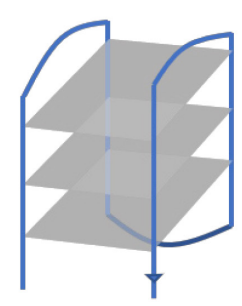

C

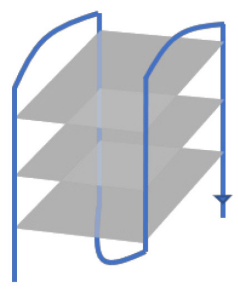

Figure 4.2: Representation of the common G-quadruplex topologies, including A) parallel Gquadruplex, B) antiparallel G-quadruplex adopting the chair conformation, and C) antiparallel Gquadruplex containing a diagonal loop.

As one of the only aptamers with a known crystal structure and conformational change upon target binding, the thrombin binding aptamer has quickly become the most prevalently used aptamer for the development of analytical assays. Molecular beacon type sensor assays were the first reported applications for the thrombin binding aptamer, relying on the known structural change to either bring together or separate a fluorophore-quencher or fluorophore-fluorophore pair. ${ }^{181}$ This has also been extended into applications involving quantum dots and Au-nanoparticles as the fluorophore and quencher moieties. ${ }^{182-183}$

\subsubsection{Choice of aptamer for the fluorogenic click reaction sensor}

Having completed the QUAL tripartite sensor system, the goal of this project was to apply the same rationale for sensor design to a fluorogenic reaction as described in the previous chapter. One of the challenges associated with the QUAL system aptamer choice was the unknown structural change between target-bound and target unbound aptamer in solution. The basis of the tripartite sensor system is that target binding results in a disruption in the interaction between the aptamer and its complementary sequences, 
thereby slowing the reaction and permitting sensing of the aptamer target. In the case of the ochratoxin A aptamer used in the QUAL section, it is assumed that the aptamer forms a G-quadruplex upon target binding and that this would result in the inability of the aptamer to bind its complementary sequences. In designing the fluorogenic tripartite system, and the later extension into a single aptamer sensor system, it was decided that a known structure switching aptamer that was selected using a capture SELEX should be used.

Oh et al. designed an improved selection strategy for a structure switching selfreporting aptamer (SRA) using thrombin as a target. ${ }^{46}$ SRAs are designed in a such a way that they contain a known DNAzyme moiety mid-sequence in the starting library, which is inactivated by a capture sequence. This DNAzyme moiety is flanked on either side by a randomized sequence that will participate in target binding during the selection. The central DNAzyme can exist in one of two conformations: a stable duplex with an antisense strand, or a G-quadruplex structure which can be probed with the organic dye $N$-methylmesoporphyrin IX (NMM). ${ }^{184}$ By attaching a DNAzyme antisense 12-base capture probe to magnetic beads in solution, the DNAzyme region is locked in its duplex structure. Upon addition of thrombin select sequences will undergo a binding event that results in a disruption of the duplex structure through formation of the G-quadruplex, resulting in those sequences being separated from the magnetic beads and processed for amplification in subsequent selection cycles.

After eight selection rounds against human $\alpha$-thrombin, three sequences were identified after sequencing to have a high affinity for thrombin, SRA_1, SRA_2 and SRA_3 (Table 4.2). ${ }^{46}$ SRA_2 was seen to have the highest affinity and selectivity for 
thrombin, with a measured $\mathrm{K}_{\mathrm{d}}$ of $93.6 \mathrm{nM}$ by RT-PCR. ${ }^{46}$ These aptamers for thrombin were said to be self-reporting based on the known DNAzyme sequence forming a Gquadruplex on target binding, which can produce a fluorescence readout upon interaction with NMM dye. ${ }^{184}$ The system was also demonstrated to work in serum as well as buffer.

Table 4.2: Aptamer sequences identified by $O$ et al. for bindng to human $\alpha$ thrombin. ${ }^{46}$

\begin{tabular}{c|c} 
& Aptamer Sequence \\
\hline SRA_1 & ACTCGGGGCGTCAAAAATTTTGTGGGTAGGGTGGGTTGGTTTTAGCACAGAGTTGTGGAT \\
SRA_2 & GAACGGGGCAAGCAAAATTTTGTGGGTAGGGTGGGTTGGTTTTGCGGAATACCGTGAGAT \\
SRA_3 & GAGAAGGCGCAGAAAATTTTTGTGGGTAGGGTGGGTTGGTTTTATGCGCGATATGGTGAA
\end{tabular}

\subsubsection{Sensor design rationale for tripartite system}

Using the SRA_2 aptamer selected by Oh et al., a tripartite sensor for thrombin based on fluorogenic click chemistry can be designed. ${ }^{46}$ As described in chapter 3 , a reaction between an alkyne-coumarin modified DNA sequence and an azide modified DNA sequence will result in the formation of a triazole-coumarin fluorophore which can be readily detected. By designing a system wherein, the SRA_2 aptamer acts as the template strand with the alkyne-coumarin, and azide labeled sequences complementary to it, the presence of thrombin can be detected based on a lower fluorescence output from the fluorogenic reaction at a given time point from inhibition of the reaction by the target. In the presence of thrombin, the aptamer will undergo a shape change upon binding to produce a G-quadruplex. The advantage of using a known structure switching aptamer, is that the complementary reactive probes can be made to match the capture probe sequence from the selection. It is known that the capture probe sequence does not remain hybridized to the 
SRA_2 aptamer upon target binding due to the formation of the G-quadruplex in the DNAzyme region. This allows the reaction between the alkyne and coumarin to be turned off, or slowed, based on the presence of thrombin in solution as depicted in Figure 4.3.
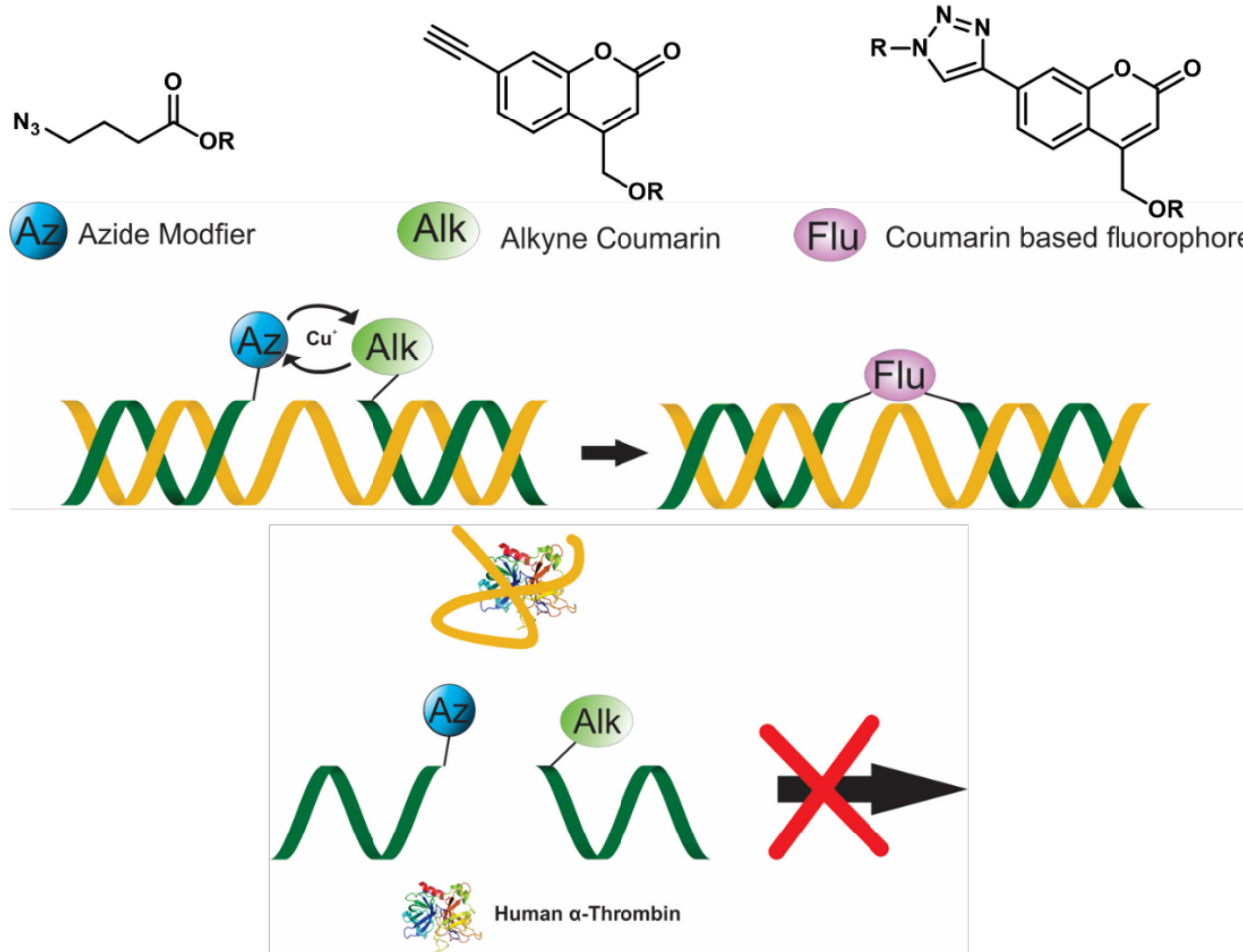

Figure 4.3: Representation of the thrombin fluorogenic sensor, with the alkyne-coumarin and azide modifier probes depicted in green and the thrombin SRA_2 aptamer in yellow. In the presence of $\mathrm{Cu}^{1}$ and absence of thrombin, a triazole-coumarin fluorophore will form and give a detectable signal, but as shown on the bottom in the presence of thrombin fluorophore synthesis is slowed.

\subsubsection{Sensor design rationale for single aptamer system with internal modifiers}

One of the drawbacks of both the QUAL system and the previously described thrombin aptamer tripartite system is that both systems result in a signal off reaction to indicate the presence of ochratoxin A or thrombin, respectively. These tripartite systems 
cannot be easily converted into signal-on sensors, and therefore alternate strategies need to be explored. One potential method for the design of a signal-on sensor using an aptamer is the direct incorporation of the reactive elements into the DNA aptamer sequence. In this strategy, reactive modifiers will not be in proximity within the sequence in the absence of the aptamer-target, but the addition of the aptamer-target will result in a conformational change that will bring the two spatially isolated groups into proximity, permitting the reaction. Such a strategy could be adopted for the sensing of thrombin using the same SRA_2 aptamer with the known DNAzyme moiety mid-sequence. By incorporating the reactive groups on either side of the DNAzyme sequence, they will be spatially isolated in the presence of the capture probe due to the duplex formed. In the presence of thrombin, the capture probe interaction will be disrupted through the formation of a G-quadruplex. Rational design of the location of the modifiers in the aptamer sequence will allow the Gquadruplex formed to bring the two modifiers into close enough proximity to allow the fluorogenic copper catalyzed reaction to occur. This will result in the formation of a fluorophore only in the presence of thrombin, thereby lowering the background and increasing the sensitivity to thrombin. An overview of the single aptamer thrombin sensor with dual internal modifiers is shown in Figure 4.4. 
A

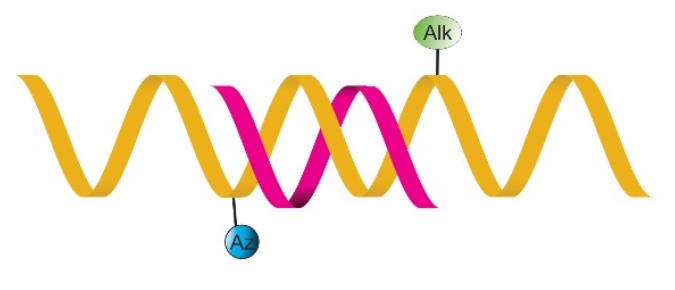

B

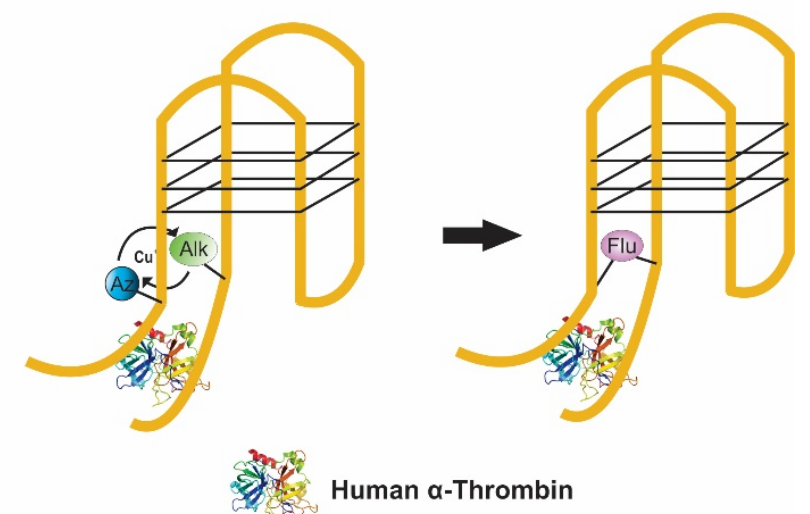

Figure 4.4: Representation of the single aptamer thrombin sensor, depicting the incorporation of the alkyne-coumarin profluorphore and the azide modfier on each side of the G-quadruplex structure. A) In the absence of target the double modified thrombin aptamer is bound to its capture probe (pink), and the alkyne and azide are kept far apart. $\mathrm{B}$ ) In the presence of human $\alpha$-thrombin and a $\mathrm{Cu}^{\mathrm{I}}$ source, the coumarin-triazole fluorophore will form after the G-quadruplex shape change brings the two modifiers into proximity, indicating the presence of thrombin.

\subsection{Experimental}

\subsubsection{Materials and equipment}

All oligonucleotide sequences were synthesized using a BioAutomation Mermade 6 oligonucleotide synthesizer using the solid phase phosphoramidite method. Azidobutryate NHS ester, 3'-amino modifier C7 CPG, amino-modifier C6-dT phosphoramdite, NHS-carboxy-dT phosphoramidite, Pac-dA-CE phosphoramidite, iPrPac-dG-CE phosphoramidite, 5\% phenoxyacetic anhydride in THF Cap Mix A, and 0.05M potassium carbonate in methanol were purchased from Glen Research. All necessary reagents for DNA synthesis including standard phosphoramidites, activator, deblock, oxidizer, capping agents, and activator were purchased from Glen Research. Standard controlled pore glass $(\mathrm{CPG})$ columns were purchased from Bioautomation. Biosynthesis 
grade acetonitrile was purchased from EMD Millipore for use on the DNA synthesizer. Ultra-high purity (UHP) argon was purchased from Praxair for use on the DNA synthesizer. Glen Pak DNA purification cartridges were purchased from Glen Research. Carbonyl diimidazole (CDI), copper(II) sulftate pentahydrate, and (+)-sodium-L-ascorbate were purchased from Sigma Aldrich. All buffers were prepared using EMD Millipore Milli-Q deionized water, and buffer salts were purchased from Sigma Aldrich and BioShop Canada Inc. Buffers were filtered through Corning 0.22 $\mu \mathrm{m}$ PES vacuum filtration systems from Sigma Aldrich. Acrylamide/bis-acrylamide 40\% solution, electrophoresis grade ammonium persulfate (APS), N,N,N',N'-tetramethylethylenediamine (TEMED), ethylenediaminetetracetic acid (EDTA), boric acid, tris(hydroxymethyl) aminomethane (Tris), and urea were purchased from Bioshop Canada Inc. Human $\alpha$-thrombin was purchased from Haematologic Technologies Incorporated.

\subsubsection{Buffer}

Table 4.3: Buffer composition for both the tripartite and single sequence thrombin fluorogenic sensor projects.

\begin{tabular}{c|c|c} 
Buffer name & Composition & $\mathrm{pH}$ \\
\hline \multirow{3}{*}{ Thrombin buffer } & $100 \mathrm{mM} \mathrm{Tris}$ & \\
& $140 \mathrm{mM} \mathrm{NaCl}$ & 7.4 \\
& $20 \mathrm{mM} \mathrm{KCl}$ &
\end{tabular}




\subsubsection{DNA synthesis, purification, and quantification for thrombin tripartite}

system

Table 4.4: Oligonucleotide sequences used in the fluorogenic thrombin tripartite click experiment. The abbreviation "Coum" is used to indicate the location of the alkyne-coumarin DNA modifier, while the $\mathrm{N} 3$ label is used to indicate the location of the azide modifier.

\begin{tabular}{c|c} 
Oligonucleotide & Sequence \\
\hline SRA_2 aptamer (S) & 5'-GAA-CGG-GGC-AAG-CAA-AAT-TTT- \\
& GTG-GGT-AGG-GTG-GGT-TGG-TTT- \\
SRA_2 coumarin (C) & TGC-GGA-ATA-CCG-TGA-GAT-3' \\
SRA_2 azide (A) & 5'-Coum-CTA-CCC-ACA-AA-3' \\
& 5'-CCA-ACC-CAC-N3-3'
\end{tabular}

The SRA_2 aptamer was synthesized under standard DNA synthesis conditions recommended by Bioautomation for the MerMade 6 DNA synthesizer, using 3'-dT CPG $1000 \AA$ columns. The SRA_2 azide was synthesized initially as its amine modified analogue using 3'-amino-modifier C7 CPG $1000 \AA$ columns under normal DNA synthesis conditions. Both the SRA_2 aptamer and the SRA_2 azide (currently amine modified) were synthesized with the final deprotection step omitted to retain the terminal DMT group for Glen Pak reverse phase cartridge purification. The SRA_2 coumarin was prepared under ultramild DNA synthesis conditions on the Bioautomation Mermade 6 DNA synthesizer. $1 \mu \mathrm{mol}$ 5'-DMT-dA $1000 \AA$ columns were used for the synthesis. The machine was switched to use ultramild reagents including Pac-dA-CE phosphoramidite for all adenine bases, ipr-Pac-dG-CE for all guanine bases, and ultramild Cap Mix A (5\% phenoxyacetic anhydride in THF) in place of the standard Cap Mix A. The alkynecoumarin phosphoramidite from chapter 3 was prepared fresh on the day of DNA synthesis 
and resuspended in acetonitrile at $0.067 \mathrm{M}$ concentration and placed in one of the modifier positions on the synthesizer. The coupling time for the alkyne-coumarin modifier was extended to 10 minutes, and the final deprotection step was omitted based on the location of the alkyne-coumarin in the DNA sequence.

Upon synthesis completion, the synthesis columns for all probes were removed from the DNA synthesizer, and the contained CPG support beads were transferred into a microcentrifuge tube. For the SRA_2 azide probe (currently amino modified) and the SRA_2 aptamer the CPG beads were deprotected in $1 \mathrm{~mL}$ of $28 \% \mathrm{NH}_{4} \mathrm{OH}$ for 16 hours at $55^{\circ} \mathrm{C}$, before proceeding to Glen Pak purification. For the coumarin SRA_2 probe, the CPG beads were deprotected for 4 hours at room temperature in $0.05 \mathrm{M} \mathrm{K}_{2} \mathrm{CO}_{3}$ in methanol before proceeding to Glen Pak desalting.

\subsubsection{Glen Pak DNA purification}

The amine modified SRA_2 azide probe as well as the SRA_2 aptamer were subjected to standard Glen Pak purification on a Glen Pak cartridge. The CPG deprotection solution in $28 \% \mathrm{NH}_{4} \mathrm{OH}$ was decanted into a new microcentrifuge tube and the beads were washed with $100 \mathrm{mg} / \mathrm{mL} \mathrm{NaCl}$ in deionized water, before decanting and mixing with the initial deprotection solution. One Glen Pak cartridge for each of the six synthesis columns

was loaded onto a Phenomenex 12 port vacuum manifold with an attached oil-free vacuum pump and rinsed with $0.5 \mathrm{~mL}$ of acetonitrile followed by $1 \mathrm{~mL}$ of $2.0 \mathrm{M}$ triethyammonium acetate (TEAA). The ammonium hydroxide/ $\mathrm{NaCl}$ deprotection solution was loaded onto each column in turn $1 \mathrm{~mL}$ at a time, and then rinsed twice with $1 \mathrm{~mL}$ of $5 \%$ acetonitrile in 
$100 \mathrm{mg} / \mathrm{mL}$ sodium chloride. The columns were then deprotected with two $1 \mathrm{~mL}$ aliquots of freshly prepared $2 \%$ trifluoroacetic acid in deionized water, at which point a bright orange band was observed. Rinsing each column twice with $1 \mathrm{~mL}$ of deionized water removed the orange bad. The collection tubes under the vacuum manifold were then switched, and the target sequence eluted using a $1 \mathrm{~mL}$ solution of $50 \%$ acetonitrile in deionized water with $0.5 \%$ ammonium hydroxide. The eluted samples were then dried on a Savant AES2010 SpeedVac cryopump overnight before resuspension in deionized water and quantification.

For the SRA_2 coumarin probe, a Glen-Pak desalting protocol was followed, wherein the potassium carbonate in methanol deprotection solution was diluted to $20 \mathrm{~mL}$ in 0.1 M TEAA. The cartridges were prepared as before, and the diluted deprotection solution was added to each of the columns. After loading, each of the columns was rinsed twice with $1 \mathrm{~mL}$ of deionized water. The collection tubes were switched for each of the columns, and the cocaine coumarin probe eluted in $1 \mathrm{~mL}$ of $50 \%$ acetonitrile in deionized water. The eluted samples were then dried on a Savant AES2010 SpeedVac cryopump overnight before resuspension in deionized water and preparation for polyacrylamide gel electrophoresis.

\subsubsection{Incorporation of the azide modifier}

The azide was incorporated onto the amine labeled SRA_2 azide probe using azidobutryate NHS-ester. The azidobutyrate NHS-ester was dissolved in $60 \mu \mathrm{L}$ of DMSO to a concentration of $0.17 \mathrm{M}$. $500 \mathrm{nmol}$ of the amine modified sequence was dissolved in 
$250 \mu \mathrm{L}$ of $0.5 \mathrm{M} \mathrm{Na}_{2} \mathrm{CO}_{3} / \mathrm{NaHCO}_{3}$ buffer $\mathrm{pH}$ 8.75. All $60 \mu \mathrm{L}$ of the azidobutyrate NHSester solution were added to the amino modified sequence in buffer, and the reaction was run for 4 hours at room temperature. The reaction was stopped through the addition of 4 $\mathrm{mL}$ of $0.1 \mathrm{M}$ TEAA, and then desalted according to the previously describe Glen Pak desalting protocol.

\subsubsection{Polyacrylamide gel electrophoresis}

The SRA_2 coumarin probe was purified using a 19\% denaturing polyacrylamide gel. The gels were prepared by mixing $63 \mathrm{~g}$ of urea with $75 \mathrm{~mL}$ of acrylamide solution (acrylamide/bisacrylamide $40 \%$ solution), and $30 \mathrm{~mL}$ of $5 \mathrm{x}$ TBE buffer ( $45 \mathrm{mM}$ Tris, 45 $\mathrm{mM}$ boric acid, $1 \mathrm{mM}$ EDTA). The solution was stirred while heating to $37^{\circ} \mathrm{C}$ and filtered while hot through Whatman No. 1 filter paper. Once cooled to room temperature, $900 \mu \mathrm{L}$ of $10 \%$ ammonium persulfate and $70 \mu \mathrm{L}$ of TEMED were added to the stirred solution. The gel solution was then poured into the casting apparatus for the Hoefer SE600 Chroma standard dual-cooled gel electrophoresis assembly. The gels were pre-run for 20 minutes using a constant voltage of 300 volts. During the pre-running, the dried dabsyl probe samples were resuspended in $300 \mu \mathrm{L}$ of deionized water, and then $300 \mu \mathrm{L}$ of formamide was added to each sample. The samples were heated to $55^{\circ} \mathrm{C}$ for 5 minutes before loading into the gel, with each column of synthesized DNA having its own gel. The gels were run for 1.5 hours, before imaging on a $F_{254}$ TLC plate in the Alpha Imager gel cabinet set on the epi-UV $(\lambda=254 \mathrm{~nm})$ and fluorescence settings $\left(\lambda_{\text {ex }}=302 \mathrm{~nm}\right)$. The relevant band for the SRA_2 coumarin probe was manually excised from the gel and placed in a $50 \mathrm{~mL}$ Falcon tube. The probe was eluted from the gel using a crush and soak method using deionized water and incubated on 
a New Brunswick Scientific Innova 40 incubator/shaker at $37^{\circ} \mathrm{C}$ for 48 hours.

After extraction, the solution was filtered through $0.22 \mu \mathrm{m}$, low retention, cellulose acetate syringe filters. The samples were then made up to $20 \mathrm{~mL}$ in $0.1 \mathrm{M}$ TEAA, and desalted according to the previously described Glen Pak desalting protocol.

\subsubsection{DNA quantification}

All three sequences were quantified by measuring their optical absorbance at 260 $\mathrm{nm}$ when dissolved in deionized water. By considering the molar extinction coefficients as determined through nearest neighbour analysis and the molar absorptivity of each of the modifiers used, an extinction coefficient can be determined. Using the Beer-Lambert law, the solution concentration of the DNA can be back-calculated. Post quantification, all DNA sequences were submitted for electrospray mass spectrometry for molecular weight confirmation by Novatia LLC. Mass spectrometry was performed using a reverse phase HPLC with direct spray into an LTQ-Orbitrap with deisotoping and deconvolution of the results undertaken using Promass software.

\subsubsection{Tripartite system ligation testing}

The thrombin tripartite fluorogenic reaction was evaluated by gel electrophoresis to determine the extent of probe ligation. In these reactions, the SRA_2 azide probe, SRA_2 aptamer, and SRA_2 probe were combined at 10 micromolar concentration each in the thrombin reaction buffer, with $2 \mathrm{mM} \mathrm{CuSO}_{4}$ and $4 \mathrm{mM}$ sodium ascorbate at a 100 $\mu \mathrm{L}$ total reaction volume. The reactions were run for 2 hours at room temperature with 
mixing every 20 minutes. After the 2-hour reaction time, all the samples were diluted with $100 \mu \mathrm{L}$ of formamide and loaded into separate lanes of a 19\% PAGE. Gels were run at $300 \mathrm{~V}$ constant for 1.5 hours, and then imaged under UV-light in the Alpha Imager to determine the extent of the reaction.

\subsubsection{Tripartite sensor evaluation with thrombin}

Final testing for the tripartite fluorogenic ligation in the presence of thrombin was performed at $10 \mu \mathrm{M}$ concentration of each probe in the presence of a wide range of thrombin concentrations. This protocol involved the incorporation of the click ligand THPTA, which was mixed with the copper sulfate solution in a 1:1 ratio such that the final concentration in the reaction mixture of each salt is $250 \mu \mathrm{M} \mathrm{CuSO}_{4} / \mathrm{THPTA}$ and $400 \mu \mathrm{M}$ sodium ascorbate. A thrombin dilution series was prepared between $100 \mathrm{nM}$ and $100 \mathrm{fM}$ in thrombin sensor buffer. The testing protocol entailed first heating each of the probes to $55^{\circ} \mathrm{C}$ then cooling to room temperature. The coumarin SRA_2 probe was then added to the thrombin buffer along with the copper sulfate-click ligand solution, before the SRA_2 azide probe was added. The reaction was initiated through the addition of the sodium ascorbate solution and allowed to proceed for 1 hour before quenching with $100 \mu \mathrm{L}$ of formamide and heating to $55^{\circ} \mathrm{C}$. Reactions were then analyzed by fluorescence spectroscopy on a Fluorolog3. From each reaction, $70 \mu \mathrm{L}$ was loaded into a quartz fluorescence cuvette, and the emission spectrum measured between 350 and $500 \mathrm{~nm}$ with an excitation wavelength of $333 \mathrm{~nm}$ and $3 \mathrm{~nm}$ slit widths. 


\subsubsection{DNA synthesis, purification, and quantification for the thrombin single aptamer system}

Table 4.5: Oligonucleotide sequences used in the fluorogenic thrombin single aptamer click experiment. The abbreviation "coum" is used to indicate the location of the alkyne-coumarin DNA modifier, while the $\mathrm{N}$ label is used to indicate the location of the azide modifier.

\begin{tabular}{c|c} 
Oligonucleotide & Sequence \\
\hline SRA_2 double modified aptamer & 5'-GAA-CGG-GGC-AAG-CAA-AAT-TTN- \\
GTG-GGT-AGG-GTG-GGT-TGG-CoumTT- \\
SRA_2 capture & TGC-GGA-ATA-CCG-TGA-GAT-3' \\
5'-ACCCACCCTACC-3'
\end{tabular}

The SRA_2 capture probe was synthesized under standard DNA synthesis conditions recommended by Bioautomation for the MerMade 6 DNA synthesizer, using 3'-dC CPG 1000 Å columns. The SRA_2 double modified aptamer was prepared under standard conditions on the Bioautomation MerMade 6 DNA synthesizer using $1 \mu \mathrm{mol} 5$ 'DMT-dT $1000 \AA$ columns. Amino modifier C6 dT phosphoramidite was suspended in acetonitrile at $0.067 \mathrm{M}$ concentration and placed in the appropriate modifier position in the sequence for the coumarin label, which is to be added post-synthesis. NHS-carboxy dT phosphoramidite was suspended in acetonitrile at $0.067 \mathrm{M}$ concentration and placed in the appropriate modifier position in the sequence for the azide label to be added post-synthesis. The coupling time for both modifiers was extended to 10 minutes, and the final deprotection step was omitted to allow for subsequent Glen Pak reverse phase cartridge purification.

Upon synthesis completion, the synthesis columns for both probes were removed from the DNA synthesizer, and the contained CPG support beads were transferred into a 
microcentrifuge tube. For the SRA_2 capture probe the CPG beads were deprotected in 1 $\mathrm{mL}$ of $28 \% \mathrm{NH}_{4} \mathrm{OH}$ for 16 hours at $55^{\circ} \mathrm{C}$, before proceeding to Glen Pak purification. For the double modified SRA_2 aptamer, the CPG beads were processed according to section 4.3.5.1 prior to deprotection under ultramild conditions.

\subsubsection{Incorporation of azide modifier on thrombin aptamer}

$0.2 \mu \mathrm{mol}$ of CPG bead support (roughly one fifth of the dry weight of CPG beads) was measured out and reacted with $2 \mu \mathrm{mol}$ of 3-azido-1-propanamine and $0.5 \mu \mathrm{L}$ of diisopropylethylamine (DIPEA) in $1 \mathrm{~mL}$ of acetonitrile for 4 hours at $37^{\circ} \mathrm{C}$. The acetonitrile was decanted, and the CPG beads were washed with $1 \mathrm{~mL}$ each of DMSO, 5\% DEA in ACN, methanol, and ACN before normal deprotection in $1 \mathrm{~mL}$ of $28 \% \mathrm{NH}_{4} \mathrm{OH}$ overnight at $55^{\circ} \mathrm{C}$.

The SRA_2 double modified probe containing the azide modifier was subjected to standard Glen Pak purification on a Glen Pak cartridge. The CPG deprotection solution in $28 \% \mathrm{NH}_{4} \mathrm{OH}$ was decanted into a new microcentrifuge tube and the beads were washed with $100 \mathrm{mg} / \mathrm{mL} \mathrm{NaCl}$ in deionized water, before decanting and mixing with the intial deprotection solution. One Glen Pak cartridge for each of the six synthesis columns was loaded onto a Phenomenex 12 port vacuum manifold with an attached oil-free vacuum pump, and rinsed with $0.5 \mathrm{~mL}$ of acetonitrile followed by $1 \mathrm{~mL}$ of $2.0 \mathrm{M}$ triethyammonium acetate (TEAA). The ammonium hydroxide $/ \mathrm{NaCl}$ deprotection solution was loaded onto each column in turn $1 \mathrm{~mL}$ at a time, and then rinsed twice with $1 \mathrm{~mL}$ of $5 \%$ acetonitrile in $100 \mathrm{mg} / \mathrm{mL}$ sodium chloride. The columns were then deprotected with two $1 \mathrm{~mL}$ aliquots 
of freshly prepared $2 \%$ trifluoroacetic acid in deionized water, at which point a bright orange band was observed. Rinsing each column twice with $1 \mathrm{~mL}$ of deionized water removed the orange bad. The collection tubes under the vacuum manifold were then switched, and the target sequence eluted using a $1 \mathrm{~mL}$ solution of $50 \%$ acetonitrile in deionized water with $0.5 \%$ ammonium hydroxide. The eluted samples were then dried on a Savant AES2010 SpeedVac cryopump overnight before resuspension in deionized water and quantification.

\subsubsection{Incorporation of coumarin modifier on thrombin aptamer}

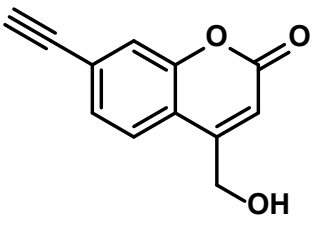

3.6

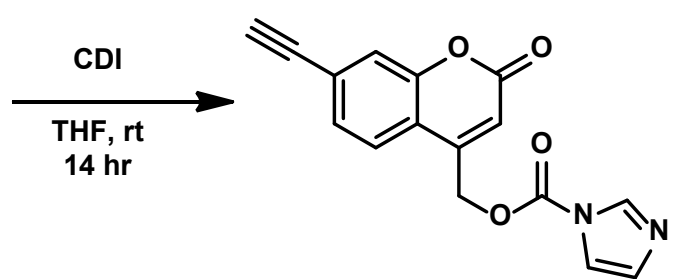

3.11

Figure 4.5: The reaction of the alkyne coumarin 3.6 with carbonyl diimidazole (CDI) to produce the CDI-activated alkyne coumarin 3.11 for addition to amine labeled DNA.

A carbonyl diimidazole (CDI) activated version of the alkyne-coumarin produced in chapter 3 was first synthesized. $5 \mathrm{mg}$ ( $32 \mu \mathrm{mol}, 1$ equiv) of the alkyne-coumarin was measured into a flame dried vial containing $16 \mathrm{mg}$ ( $98 \mu \mathrm{mol}, 3$ equiv) of CDI in $32 \mu \mathrm{L}$ of dry tetrahydrofuran (THF). The reaction proceeded for 14 hours on a vortex shaker. Evaporation of the THF under reduced pressure and resuspension of the powder in ethyl acetate and washing with water followed by concentration under reduced pressure yielded $5.1 \mathrm{mg}$ (54\% yield) of product. This CDI-activated coumarin was used in the subsequent step without further analysis. 
Reaction of the CDI-activated coumarin with the SRA_2 double modified aptamer that has already been azide-modified and purified was performed on $1 \mu \mathrm{mol}$ of the azidemodified DNA. Reacting $5 \mu \mathrm{mol}(1.5 \mathrm{mg}, 5$ equiv) of CDI-activated coumarin with $1 \mu \mathrm{mol}$ of azide-modified DNA was performed in $1 \mathrm{~mL}$ of $0.15 \mathrm{M}$ bicarbonate buffer $\mathrm{pH} 9.6$ for 4 hours at room temperature. Post-reaction, the sample was diluted to $5 \mathrm{~mL}$ in $0.1 \mathrm{M}$ TEAA and subjected to Glen Pak cartridge desalting before drying and quantifying.

\subsubsection{Sensor evaluation for thrombin single aptamer system}

Testing of the single aptamer fluorogenic reaction in the presence of thrombin was performed at a $1 \mu \mathrm{M}$ concentration of both SRA_2 double modified aptamer and SRA_2 capture probe in the presence of a wide range of thrombin concentrations. This protocol involved the incorporation of the click ligand THPTA, which was mixed with the copper sulfate solution in a 1:1 ratio such that the final concentration in the reaction mixture of each salt is $250 \mu \mathrm{M} \mathrm{CuSO}_{4} / \mathrm{THPTA}$ and $400 \mu \mathrm{M}$ sodium ascorbate. A thrombin dilution series was prepared between $100 \mathrm{nM}$ and $100 \mathrm{pM}$ in thrombin sensor buffer. The double modified SRA_2 aptamer was added to thrombin buffer along with the SRA_2 capture probe and heated to $55^{\circ} \mathrm{C}$ before cooling to room temperature. The copper sulfate- click ligand solution was then added, and the reaction was initiated through the addition of the sodium ascorbate solution and allowed to proceed for 1 hour before quenching with 100 $\mu \mathrm{L}$ of formamide and heating to $55^{\circ} \mathrm{C}$. Reactions were then analyzed by fluorescence spectroscopy on a Fluorolog3. From each reaction, $70 \mu \mathrm{L}$ was loaded into a quartz fluorescence cuvette, and the emission spectrum measured between 350 and $500 \mathrm{~nm}$ with 
an excitation wavelength of $333 \mathrm{~nm}$ and $3 \mathrm{~nm}$ slit widths.

\subsection{Results and Discussion}

\subsubsection{Tripartite thrombin sensor system}

\subsubsection{Tripartite thrombin capture probe design rationale}

The SRA_2 thrombin aptamer was chosen as the aptamer of choice for catalyzing a controllable fluorogenic click ligation because of its known structure switching properties. Unlike the ochratoxin A aptamer 1.12.2 used in the QUAL study, the SRA_2 aptamer was selected through a capture SELEX method for the formation of a Gquadruplex in the DNAzyme region of the aptamer sequence. Upon thrombin target binding, the interaction between the DNAzyme region and the complementary capture probe is disrupted, a property that could be advantageous in the production of a tripartite sensor as was proposed for the controllable fluorogenic click ligation. If the fluorogenic click handles were installed on sequences equivalent to half of the capture probe, it can be assumed that upon target binding these capture split probes will not bind to the aptamer. This would result in the reaction between the click handles being delayed relative to if the target was not present, allowing for a controllable system. In the QUAL project, it was assumed that the complementary sequences would bind the OTA 1.12 .2 aptamer in the presence of target. That assumption can be made with a more rational foundation by using a known structure switching aptamer, as it is known that the capture probe cannot bind based on the selection strategy. 
Splitting the capture probe from the selection by Oh et al. in half from its initial 12 base sequence would result in two six base probes being used to catalyze a ligation reaction. Analysis of the melting temperature using IDT OligoAnalyzer showed that 6 base complementary sequences would result in a melting temperature that would be too low for a favourable interaction at room temperature. Therefore, the two six base sequences were elongated in the 5' and 3' directions until a reasonable melting temperature was reached for each of the probes.

Table 4.6: Estimated melting temperatures for each probe based on entering the sequence into IDT OligoAnalyzer.

\begin{tabular}{c|c} 
Sequence & Melting temperature $\left({ }^{\circ} \mathbf{C}\right)$ \\
\hline Capture probe: 5'- ACCCACCCTACC-3' & 44.2 \\
5'-Capture 6 base: 5'-ACCCAC-3' & 2.4 \\
3'-Capture 6 base: 5'-CCTACC-3' & 0 \\
SRA_2 coumarin probe: 5'-CTA-CCC-ACA-AA-3' & 31 \\
SRA_2 azide probe: 5'-CCA-ACC-CAC-3' & 29.6
\end{tabular}

The designed coumarin probe would need be 12 bases in length, while the azide probe could be 9 bases in length to maintain a reasonable melting temperature at room temperature. This would likely result in a decrease in affinity of the aptamer for its thrombin target as 21 bases of complementary sequence rather than 12 bases would need to be disrupted for target binding to occur.

\subsection{Synthesis and incorporation of alkyne coumarin and azide DNA modifier}

The alkyne-coumarin profluorophore phosphoramidite from chapter 3 was added onto the 5' end of the SRA_2 coumarin probe using the DNA synthesizer and treating it as a single position modifier. Due to the unknown lability of alkyne-coumarin modifier 
ultramild DNA synthesis strategies were adopted to help protect the synthetically expensive coumarin modifier from premature degradation. Ultramild conditions consist of switching the protecting groups on adenine and guanine phosphoramidites to ones that are more easily removed using potassium carbonate in methanol deprotection at room temperature rather than ammonium hydroxide treatment overnight at $55^{\circ} \mathrm{C}$. Following the two-step purification of the alkyne coumarin by Glen Pak desalting and polyacrylamide gel electrophoresis, a reasonable yield of $600 \mathrm{nmol}$ of modified DNA off of six $1 \mu \mathrm{mol}$ synthesis columns was obtained. Electrospray-ionization mass spectrometry (ESI-MS) molecular weight confirmation was performed post purification, and the product was confirmed with a molecular weight of $3516.6 \mathrm{~g} / \mathrm{mol}$ with the alkyne-coumarin modifier (appendix figure C.1).

The SRA_2 azide probe was synthesized over two steps starting from a 3'-amine modification that could then be converted to an azide. The 3'-amine modification was incorporated through the use of 3'-amino C7 CPG columns, and purified by Glen-Pak cartridge purification with a yield of $1.4 \mu \mathrm{mol}$ from six synthesis columns. The higher yield than the coumarin modifier is expected due to the shorter sequence as well as the absence of modifier apart from the already installed 3' modification on the column and the non-ultramild conditions. ESI-MS molecular weight confirmation showed the successful synthesis of the SRA_2 amine with a molecular weight of $2822 \mathrm{~g} / \mathrm{mol}$ (appendix figure C.2). The azide modifier was incorporated by reacting the amino modified sequence with azidobutryate NHS-ester with a yield of $300 \mathrm{nmol}$ from 1 micromol of starting material. ESI-MS molecular weight confirmation showed the expected $2933.1 \mathrm{~g} / \mathrm{mol}$ molecular 
weight for the SRA_2 azide (appendix figure C.3).

The SRA_2 aptamer was also synthesized without any modification from the literature sequence with no modifications in the synthesis strategy. A yield of $2 \mu \mathrm{mol}$ of purified DNA was obtained following Glen Pak cartridge purification. ESI-MS molecular weight confirmation demonstrated the expected molecular weight of $18826.7 \mathrm{~g} / \mathrm{mol}$ (appendix figure C.4).

\subsubsection{Demonstration of OTR functionality in the absence of thrombin}

To demonstrate the ligation between SRA_2 azide and SRA_2 alkyne in the presence of the SRA_2 aptamer, a series of test reactions in the absence of thrombin were performed and loaded onto separate lanes of a denaturing gel. As can be seen in Figure 4.6, lanes containing only one type of DNA (C, A, or S) showed only a single band as expected. In the three reaction lanes containing all three sequences mixed together (CAS), a new product band is observable at the midpoint of the lane below the $\mathrm{S}$ aptamer band and above the azide and alkyne bands that were ligated together to form the new product. Interestingly, in this experiment a slight background reaction between the SRA_2 azide and SRA_2 alkyne could be observed in the CA lane as the presence of a band similar to the reaction lanes but of much lower intensity. Monitoring of the background reaction between the azide and alkyne labeled probes became a priority from this point when analyzing the fluorescence spectra to ensure that there is minimal interference. This was believed to be caused by a concentration effect- $10 \mu \mathrm{M}$ reaction concentration for each of the probes may be sufficient for an intermolecular reaction in this highly efficient form of 
click chemistry. To avoid this in the future, reaction times were decreased from the 2 hours that this experiment was run for in order to avoid the possible background effect seen here.

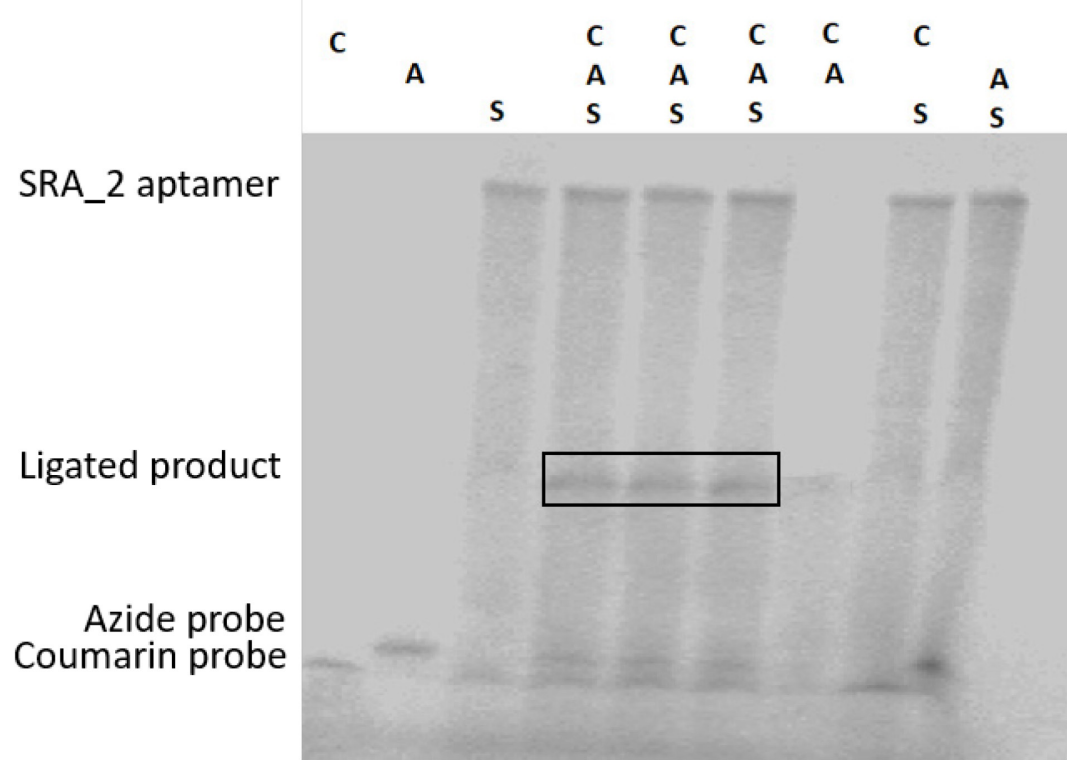

Figure 4.6: Denaturing 19\% polyacrylamide gel electrophoresis run on $10 \mu \mathrm{M}$ reaction between SRA_ 2 coumarin (C), SRA_ 2 azide (A), and SRA_2 aptamer (S) loaded into separate wells. Ligated thrombin product is only observable in lanes containing all three sequences (CAS) and is indicated by the black box. The identity of the bands in the gel is indicated by the label on the left, corresponding to the distance travelled in the gel.

\subsubsection{Detection of thrombin with the OTR sensor system}

For the tripartite thrombin sensor reaction, a comparison between the fluorescence emission spectrum of all three sequences mixed together in the presence and absence of human $\alpha$-thrombin will allow for the signal-off detection of thrombin. When the SRA_2 aptamer is mixed with the complementary SRA_2 azide and SRA_2 coumarin probes (reaction labeled as CAS), a fluorogenic ligation reaction will occur between the azide and alkyne in the presence of a copper(I) source. Upon addition of thrombin, a structural change will occur in the SRA_2 aptamer, resulting in the formation of a G-quadruplex and 
a decrease in the ability of the coumarin and azide labeled probes to bind. This will result in a decrease in the maximum fluorescence emission at the expected $410 \mathrm{~nm}$ wavelength for the coumarin fluorophore formed in the reaction. The minimum fluorescence intensity of the system will be that found in the background reaction consisting of either the SRA_2 coumarin sequence alone, or in the presence of the SRA_2 aptamer or SRA_2 azide individually. The fluorescence emission peak in the presence of thrombin will be found in between the maximum peak for the reaction in the absence of thrombin (CAS), and the background reaction $(\mathrm{C}, \mathrm{CA}, \mathrm{CS})$. Much like the QUAL system previously described, this system is limited by the fluorescence intensity difference between these two sets of peaks. Higher concentrations of thrombin should further inhibit the formation of the fluorophore and thereby result in a fluorescence emission nearing the background signal for those reactions. Lower thrombin concentrations will result in higher fluorescence emissions approaching the CAS reaction.

An experiment was performed as shown in Figure 4.7, wherein the fluorescence emission spectrum between $350 \mathrm{~nm}$ and $500 \mathrm{~nm}$ with an excitation of $330 \mathrm{~nm}$ was measured for the background reactions, the main reaction (CAS) and the reactions in the presence of a range of thrombin concentrations. For this test, a thrombin dilution series between $100 \mathrm{nM}$ and $100 \mathrm{fM}$ was prepared. The expected trend was observed in Figure 4.7, with the background SRA_2 coumarin containing reactions providing the lowest signal, and the CAS reaction providing the highest signal. The reactions in the presence of thrombin are found in between these two groups of signals, in a decreasing order of fluorescence intensity from lowest concentration to highest concentration. It also appears 
that in the presence of thrombin the fluorescence emission peak at $410 \mathrm{~nm}$ appears to broaden.

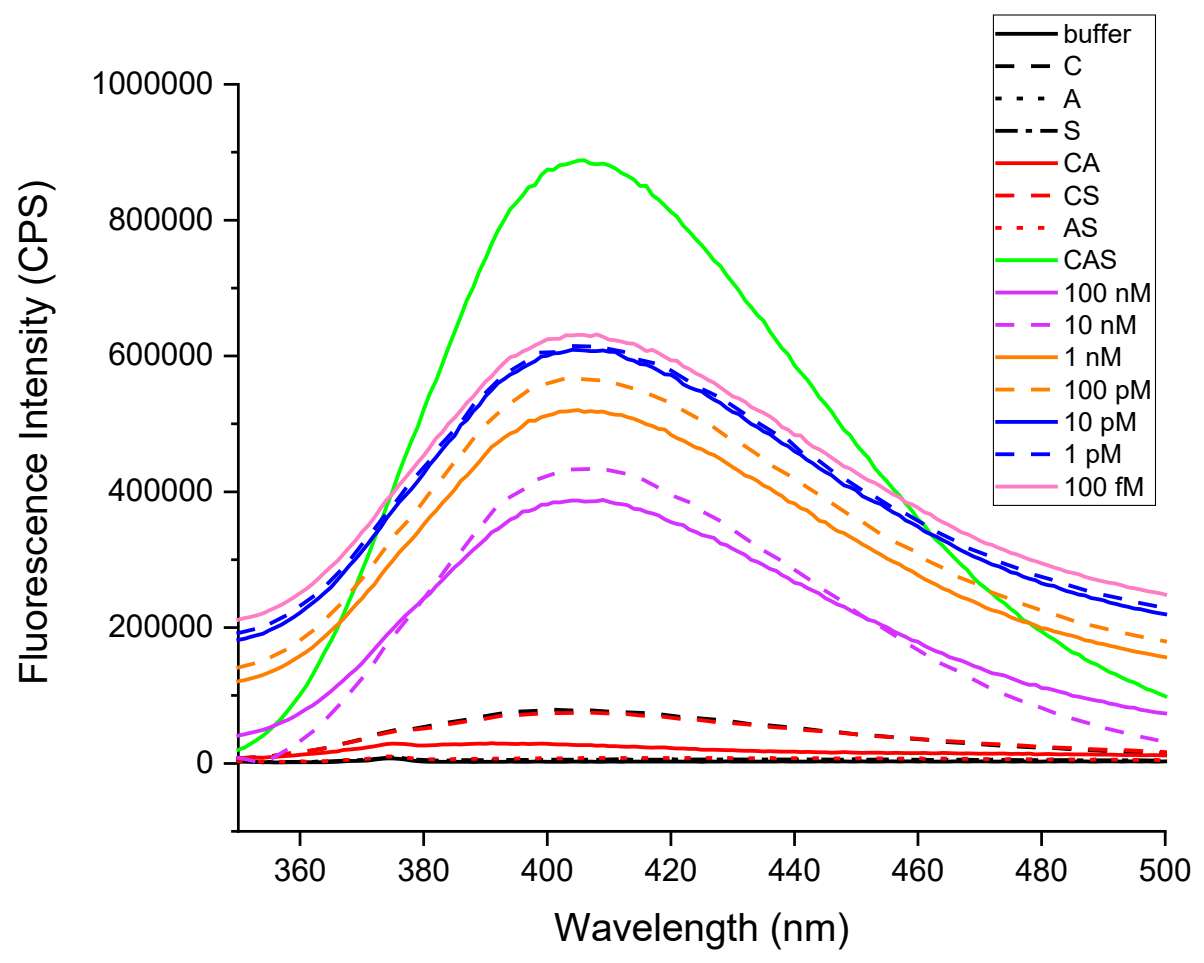

Figure 4.7: Fluoresence emission spectra measured between $350-500 \mathrm{~nm}$ with an excitation of $333 \mathbf{~ n m}$. Reactions consisted of a mixture of SRA_2 coumarin (C), SRA_2 azide (A) and SRA_2 aptamer (S), in the presence of $\mathrm{Cu}(\mathrm{I})$ with or without the addition of thrombin along a dilution series $(100 \mathrm{nM}-100$ fM).

A simplified view of the trend at $410 \mathrm{~nm}$ is shown in Figure 4.8. A one-way ANOVA analysis was performed in order to compare the means of the data reported in Figure 4.8 , and it was determined that at $\alpha=0.05$, the population means in between samples were significantly different $[\mathrm{F}(16,33)=2682.7, \mathrm{p}=0]$. Two factor mean comparison was performed using a Tukey analysis, and at $\alpha=0.05$ there was a significant difference in the mean between all non-background reaction samples except for the $1 \mathrm{pM}$ to $100 \mathrm{fM}$ thrombin 
concentrations which are equal, indicative of a plateau. As expected CAS is the most fluorescent reaction, and all the thrombin reactions have significantly lower fluorescence intensity. The background reactions for all SRA_2 coumarin containing samples (C, CA, CS) proved to have very low fluorescence, indicating that this system requires all three probes to be present for the ligation reaction to occur. This is as expected, since minimal reaction is expected between the probes at this low concentration and speaks to the minimal side reactions associated with the azide-alkyne click chemistry and the overall stability of the modifiers. Unlike the QUAL system where rapid non-specific degradation of the dabsyl quencher and its connection to the probe resulted in high backgrounds that could potentially skew the results, no such problem is expected with the click system. Also, the fluorescence intensity of the reaction in the presence of thrombin has not appeared to bottom out to the same value as the background reactions, and therefore it is expected that the sensor can be expanded to higher concentrations of thrombin. 


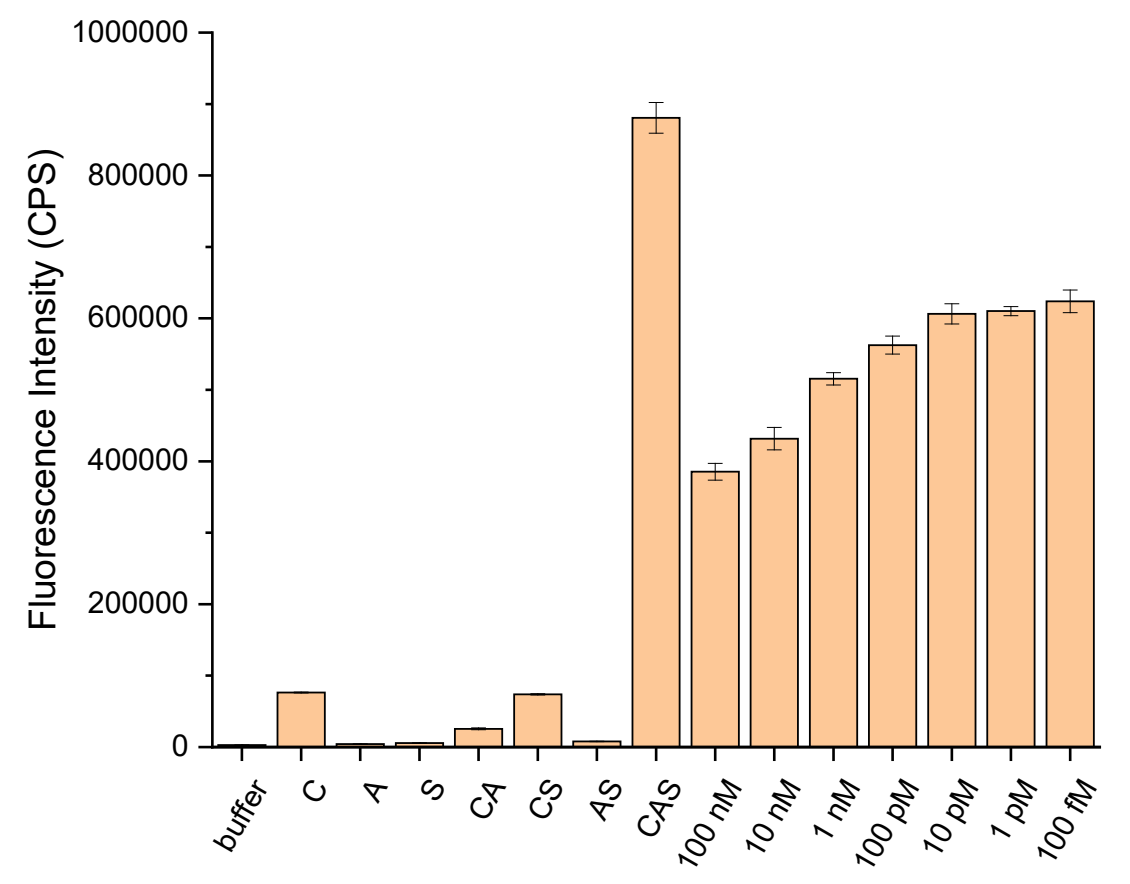

Figure 4.8: Bar plot conversion of the data from Figure 4.7 showing the measured fluorescence intensity at $410 \mathrm{~nm}$ for each of the reactions with the indicated concentration of thrombin. A linear trend in increasing fluorescence intensity with decreasing thrombin concentration is observed between $100 \mathrm{nM}$ and $10 \mathrm{pM}$ thrombin concentrations. All data was collected in triplicate $(\mathrm{N}=3)$. One way subjects ANOVA at $\alpha=0.05$ showed a significant difference between all means $[F(16,33)=2682.7$, $\mathbf{p}=\mathbf{0}]$.

Much like the data observed in the QUAL sensor system for ochratoxin A, the fluorescence intensity of the thrombin sensor appears to plateau in the presence of low concentrations of thrombin well below the fluorescence intensity of the reaction in the absence of thrombin (CAS). Hypothetically, the fluorescence intensity should match that of the CAS reaction once the thrombin concentration reaches low values. Since it does not, it would be assumed that the presence of thrombin is causing some form of extraneous effect in the system, likely because of quenching. Thrombin has nine tryptophan residues which absorb at $280 \mathrm{~nm}$ and emit surrounding $340 \mathrm{~nm} .{ }^{185}$ The role of thrombin protein in 
altering the fluorescence emission spectrum of the product warrants further investigation.

\subsubsection{Limit of detection and linear dynamic range of click thrombin sensor system}

Conversion of the plot from Figure 4.8 to a scatter plot for the $410 \mathrm{~nm}$ emission fluorescence intensity permitted an evaluation of the linear dynamic range of the system and is shown in Figure 4.9. Based on the trend observed, a linear range of thrombin effect can be demonstrated between $100 \mathrm{nM}$ and $10 \mathrm{pM}$, or five orders of magnitude, with a 100 $\mathrm{nM}$ limit of linearity. During a coagulation event thrombin concentrations vary between picomolar and micromolar, and therefore our system is within the relevant range for assessment of thrombin levels in blood. ${ }^{173}$

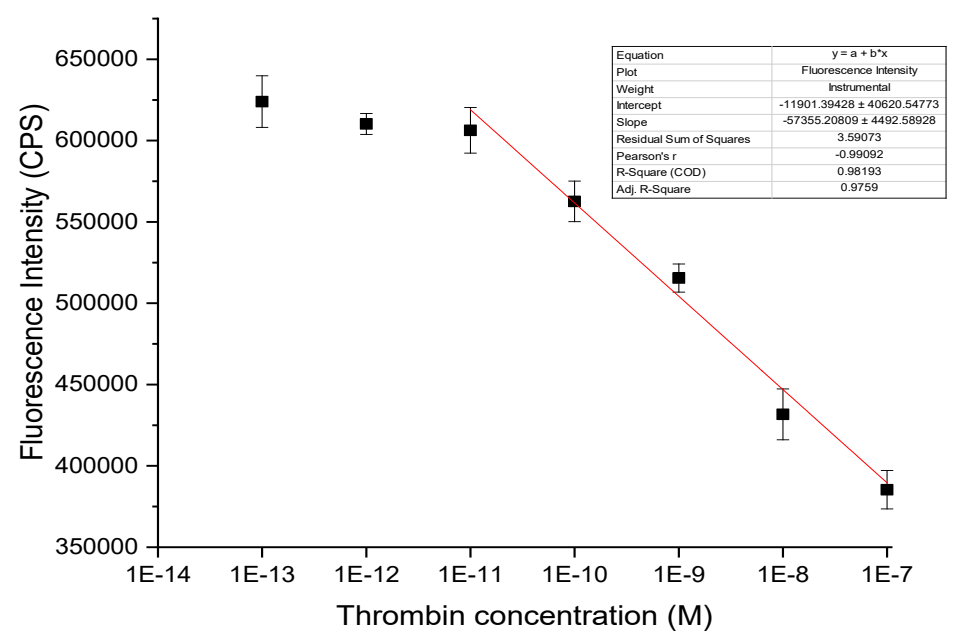

Figure 4.9: Scatter plot conversion of the observed trend at $410 \mathrm{~nm}$ emission for the reaction in the presence of $100 \mathrm{nM}$ to $10 \mathrm{pM}$ human $\alpha$-thrombin. A linear dynamic range of $100 \mathrm{nM}-10 \mathrm{pM}$ thrombin is observed.

Evaluation of the limit of detection of the thrombin tripartite aptamer system was 
performed using the standard deviation of the reaction in the absence of thrombin (CAS). By plotting the difference in the fluorescence intensity between the reaction in the absence of thrombin and at a given concentration, a linear trend was observed when plotted against the logarithm of the thrombin concentration in pM, much like that seen in Figure 4.10. Using the equation of the line shown in Figure 4.10, the limit of detection can be calculated as the concentration at which the fluorescence intensity is equal to 3 times the standard deviation of the blank buffer fluorescence. Inputting those numbers, an LOD of $3.9 \mathrm{fM}$ can be calculated. A limit of quantification can also be quantified, using 10 times the standard deviation of the blank fluorescence, as $10.6 \mathrm{fM}$.

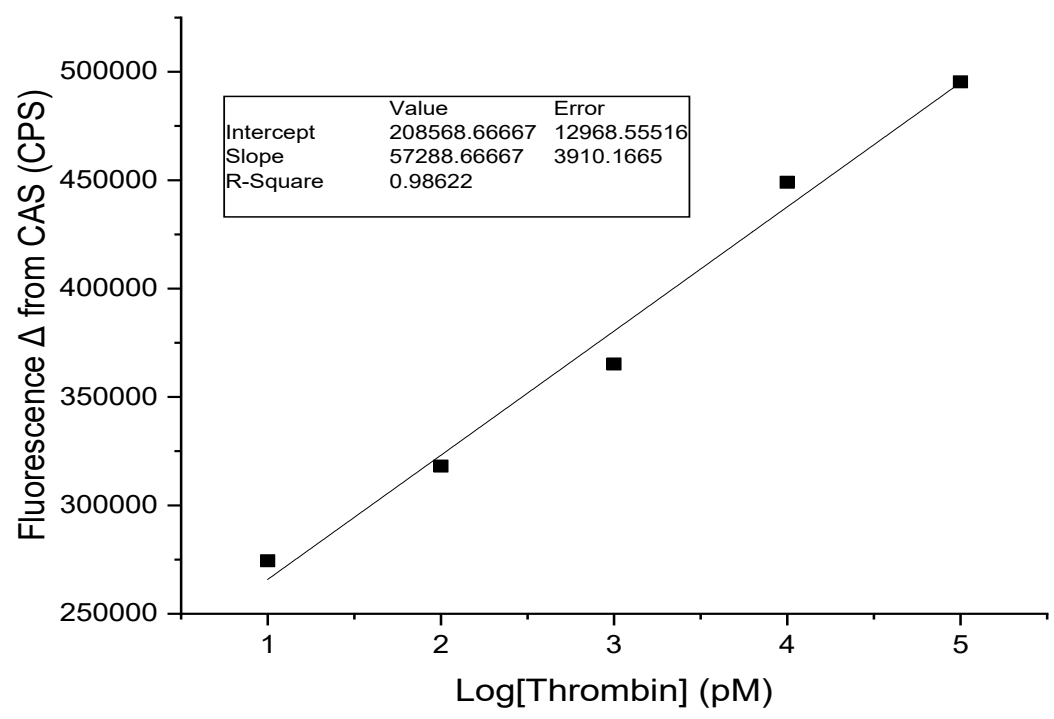

Figure 4.10: Plot of change in fluorescence from CAS for each thrombin concentration, plotted against the logarithm of thrombin concentration in pM. Using the regression analysis, an LOD of $3.9 \mathrm{fM}$ was calculated. All data was collected in triplicate $(\mathrm{N}=3)$, with error bars appearing smaller than some markers.

Many thrombin sensors have been reported in literature and can be readily 
compared to the fluorogenic tripartite system prepared herein. A thrombin aptasensor that incorporates gold nanoparticles as the reporter element demonstrated a linear range of 0.1 $-15 \mathrm{nM}$ with a limit of detection of $0.1 \mathrm{nM}$. This is much narrower of a dynamic range than that established for the tripartite sensor system and is not nearly as sensitive as the hypothetically calculated LOD reported herein. Sensor systems do exist with limits of detection approaching those seen here, mostly based on electrochemical aptasensors and their associated high sensitivity. One such example showed a linear dynamic range of 0.05 $\mathrm{pM}$ to $60 \mathrm{nM}$ thrombin with a limit of detection of $15 \mathrm{fM}$. This is quite like the system explored here, with a linear dynamic range that covers a wider range due to the enhanced sensitivity, but the tripartite fluorogenic thrombin system does have the advantage of a lower limit of detection. Therefore, the thrombin tripartite fluorogenic sensor system is quite relevant when compared to literature and rivals the best electrochemical sensors in terms of limit of detection and linear dynamic range. It should be noted that thrombin levels in the blood range from picomolar to micromolar following activation, and therefore any clinically relevant applications should be designed around a sensor that works in this

range as the tripartite system does. ${ }^{173}$ The advantage of the tripartite system is the ability to apply this technology to other known structure switching aptamers for the development of rapid and highly sensitive fluorescence sensors.

\subsubsection{Controls and specificity of thrombin sensor system}

The final step in evaluation of the thrombin tripartite sensor system is assessment of the reaction in the presence of controls to determine the specificity of the sensor. The 
CAS system was tested with ATP and adenosine controls, and it was observed that ATP did not have an effect on the fluorescence output at $410 \mathrm{~nm}$ but adenosine did result in a lower signal as seen in Figure 4.11. These are not the most relevant controls for this system, and future work would require the evaluation of related proteins. An alternative control test that was run was replacement of the SRA_2 azide sequence with an alternative cocaine sequence- namely the cocaine azide sequence from Chapter 6. In these reactions in the presence and absence of thrombin (CS CAZ and CS CAZ $10 \mathrm{Thr}$ respectively), minimal fluorescence signal is observed. This demonstrates the importance of the correct azide sequence for binding to the complementary SRA_2 aptamer. This is a templated ligation reaction, and a change of sequence results in minimal interaction and therefore minimal fluorogenic ligation. 


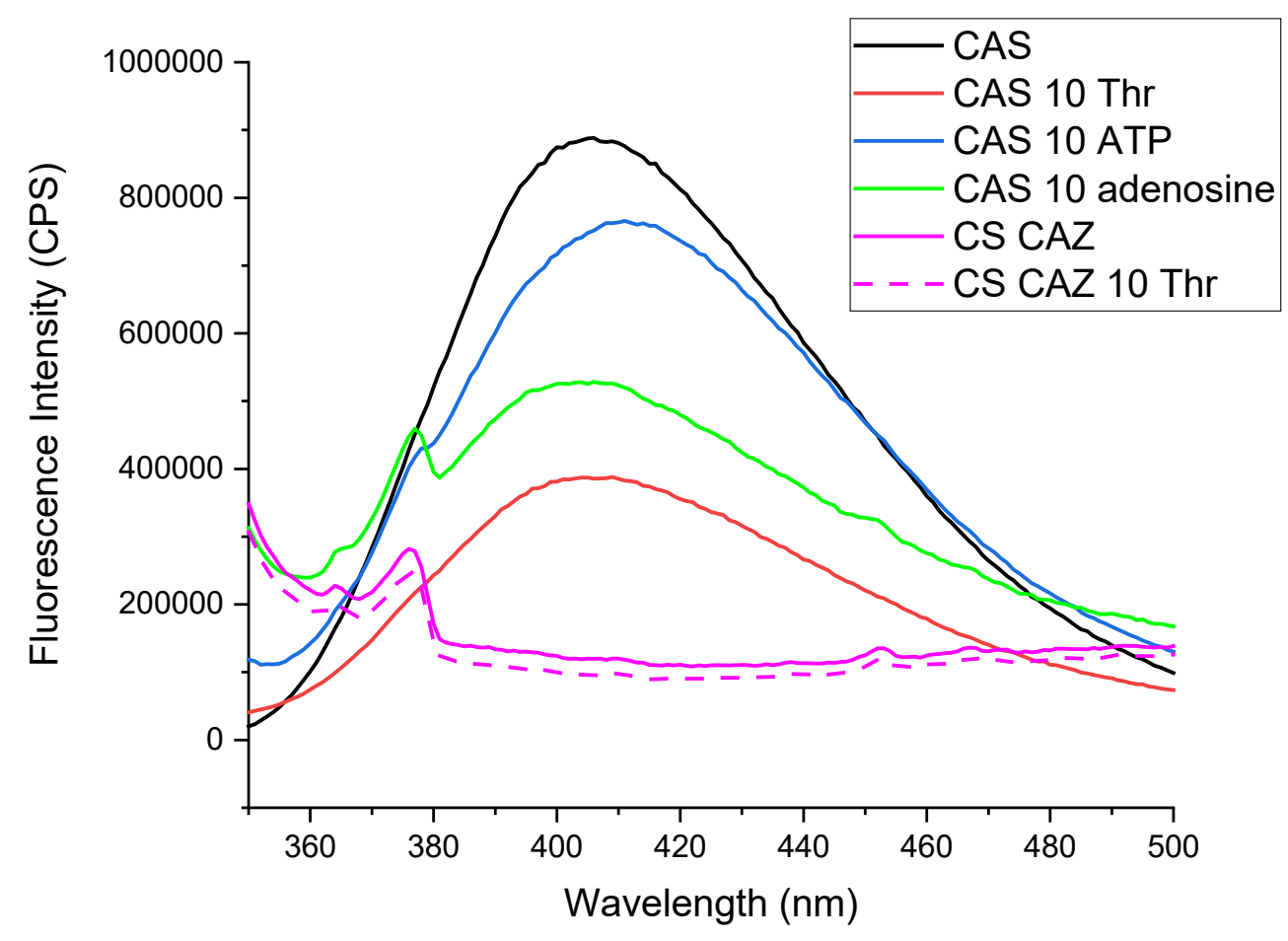

Figure 4.11: Fluorescence spectrum of the ligation reaction between the SRA_2 coumarin (C), SRA_2 azide (A), and the complementary SRA_2 aptamer (S), in the presence of $10 \mu \mathrm{M}$ concentration of adenosine, and ATP as controls. The SRA_2 azide was also replaced with the cocaine azide probe $(C A Z) . \lambda_{e x}=333 \mathrm{~nm}, \lambda_{\mathrm{em}}=350-500 \mathrm{~nm}$. Fluorescence emission of the reaction in the absence of target was higher than in the presence of all controls. Replacing the azide probe with the cocaine azide probe resulted in only background fluorescence emission in the presence and absence of thrombin (CS CAZ $10 \mathrm{Thr}$ and CS CAS respectively). 


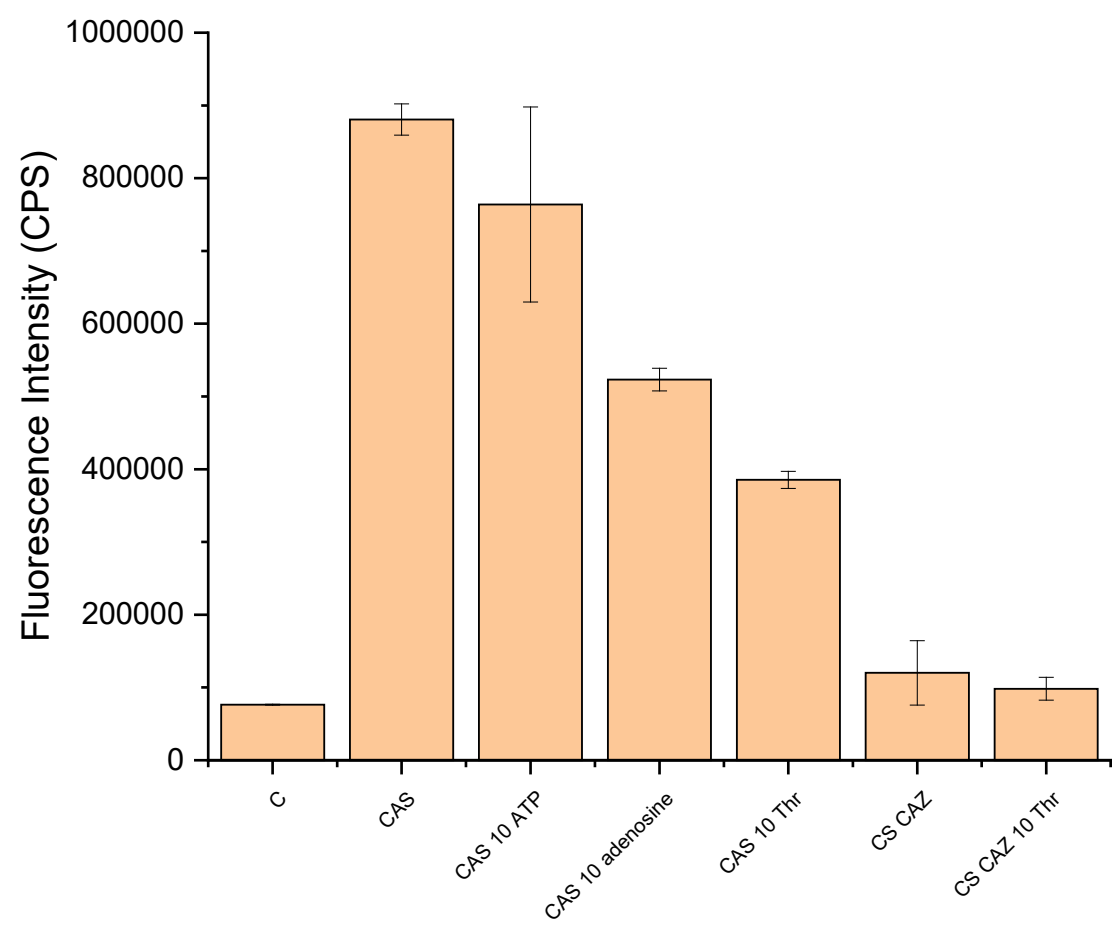

Figure 4.12: Bar graph of the data represented in Figure 4.11 at $410 \mathrm{~nm}$ demonstrating the ligation reaction SRA_2 coumarin (C), SRA_2 azide (A), and the complementary SRA_2 aptamer (S), in the presence of $10 \mu \mathrm{M}$ concentration of adenosine, and ATP as controls. The SRA_2 azide was also replaced with the cocaine azide probe (CAZ). Fluorescence emission at $410 \mathrm{~nm}$ of the reaction in the absence of target was higher than in the presence of all controls. Replacing the azide probe with the cocaine azide probe resulted in only background fluorescence emission in the presence and absence of thrombin (CS CAZ $10 \mathrm{Thr}$ and CS CAS respectively). All data was collected in triplicate $(\mathrm{N}=3)$.

\subsubsection{Single aptamer sensor system}

\subsubsection{Azide and alkyne modification location rationale for single aptamer system}

The desire to design a fluorogenic reaction that resulted in signal-on instead of signal-off prompted the exploration of a single aptamer system for the fluorogenic click reaction between an alkyne-coumarin and an azide. Since a structure switching system had already been proposed for the tripartite system, it was postulated that this structure 
switching component could be advantageous for a controllable reaction in a method like that seen in molecular beacons. When the capture probe is bound to the DNAzyme region, modifiers on either side of the DNAzyme region should be kept at a sufficient distance to inhibit the intramolecular reaction. By minimizing concentration, the intermolecular reaction can also be limited. In the design of the structure switching SRA_2 aptamer for thrombin proposed by Oh et al., there are several important regions as denoted in Figure 4.13. ${ }^{46}$ There is the central DNAzyme region that is known to form a G-quadruplex structure upon target binding and is complementary to the 12 base capture probe. On either side of the DNAzyme region there are spacers consisting of four thymine bases. On each side of the thymine base spacers is the randomized region selected during the SELEX experiment from the randomized pool.

5'-GAA-CGG-GGC-AAG-CAA-AAT-TTT-GTG-GGT-AGG-GTG-GGT-TGG-TTT-TGCGGA-ATA-CCG-TGA-GAT-3'

Figure 4.13: The SRA_2 aptamer selected by Oh et al. consisted of three distinct regions. A DNAzyme G-quadruplex forming region shown in green, a four thymine base spacer region shown in red, and the randomized regions selected for target binding shown in black.

It was therefore decided that incorporating the coumarin modifier in the $5^{\prime} \mathrm{T}$ spacer region and the azide modifier in the 3 '-T spacer region as shown in Figure 4.14, their reaction could be controlled. Upon target binding, the capture probe complementary to the DNAzyme sequence would no longer bind, and the T-spacer regions on either side of the G-quadruplex would be brought into proximity.

5'-GAA-CGG-GGC-AAG-CAA-AAT-TT-Azide-GTG-GGT-AGG-GTG-GGT-TGG- 


\section{Coumarin-TT-TGC-GGA-ATA-CCG-TGA-GAT-3'}

Figure 4.14: The modification of the SRA_2 thrombin aptamer at the indicated locations would permit the controllable reaction between the alkyne coumarin (red), and the azide (blue). The DNAzyme region is indicated in green.

\subsubsection{Incorporation of an azide modifier within the SRA_2 aptamer sequence}

To incorporate both modifiers within the sequence a strategy had to be adopted for compatible modifiers that could be sequentially reacted with either the azide or the coumarin. It was determined that the easiest method to incorporate the azide modifier would be through the reaction of 3-azido-1-propanamine with an NHS-carboxy dT modifier within the sequence immediately following DNA synthesis. No cross-reaction with the protected amino modifier being used to incorporate the alkyne-coumarin is expected. Immediately following the synthesis, the CPG beads were divided into aliquots and allowed to react with 3'-azido-1-propanamine, followed by a series of washes before deprotection of the oligonucleotide in aqueous ammonium hydroxide. The synthesis and reaction to incorporate the amine yielded $1100 \mathrm{nmol}$ off 12 synthesis columns. ESI-MS molecular weight confirmation was used to demonstrate the incorporation of the azide modifier, with the correct molecular weight of $19119.4 \mathrm{~g} / \mathrm{mol}$ for the SRA_2 aptamer sequence with the amine modifier (appendix figure C.5).

\subsubsection{Incorporation of alkyne coumarin within the SRA_2 aptamer sequence and} associated issues

The alkyne-coumarin was incorporated after the azide modifier had been incorporated by reacting the amine DNA modifier with a CDI-activated version of the 
alkyne-coumarin. It is expected that the incorporation of the CDI-coumarin occurs with relative ease in its 4-hour reaction with the amine-modified azide-containing SRA_2 aptamer in bicarbonate buffer, but this was not seen. Due to limited availability of the coumarin starting material, synthesis of the CDI-activated alkyne coumarin was performed at a low $5 \mathrm{mg}$ scale with a yield of $9 \mathrm{mg}$ of product. It is postulated that the low synthesis yield of the CDI-coumarin could be a result of the aqueous conditions resulting in preferential imidazole displacement. The synthesis of the activated coumarin ester could be improved using an alternative activated ester with enhanced aqueous stability. Due to the low yields and desire to rapidly react the DNA with the coumarin modifier the reaction was performed without a complete analysis of the product after its recovery. Reaction of a five-fold excess of the CDI-coumarin label with $1 \mu \mathrm{mol}$ of the amine labeled DNA resulted in a maximum yield of 6 nmol of DNA over three attempts at synthesis with one such reaction resulting in zero incorporation of the coumarin modifier observed through ESI-MS molecular weight confirmation. In the synthesis yielding $6 \mathrm{nmol}$ of double modified DNA, ESI-MS confirmed the incorporation of the alkyne-coumarin modifier with the expected molecular weight of $19344.6 \mathrm{~g} / \mathrm{mol}$ (appendix figure C.6).

Due to time constraints and limited starting material availability this reaction was not revisited after the three initial attempts consumed the remaining alkyne coumarin and the remaining amino-modified aptamer. It is likely that in the process of the reaction of the CDI-coumarin and subsequent washing and reverse phase cartridge purification significant loss occurred. Analysis of the flow through from the post-synthesis cartridge purification did not show significant quantities of DNA remaining, with the recovery of a 
maximum of $100 \mathrm{nmol}$ of amine labeled DNA from those collected fractions. It is also possible that the CDI-coumarin synthesized was not as pure as assumed and therefore future directions would include scaling up and studying the results of that reaction with additional alkyne-coumarin precursor synthesized. With additional starting material the incorporation of the CDI-coumarin could also be studied under a variety of conditions and purification strategies to optimize its production for further downstream testing.

\subsubsection{Fluorescence signal increase in the presence of thrombin target}

It is expected that addition of thrombin to a mixture of the double modified thrombin aptamer and its complementary capture probe would initiate a copper-catalyzed azide-alkyne cycloaddition between the alkyne-coumarin modifier and the azide modifier. As shown in Figure 4.15, increasing concentrations of thrombin resulted in an increase in the fluorescence intensity with a peak observed at the expected $410 \mathrm{~nm}$ with a $333 \mathrm{~nm}$ excitation. The highest concentration of thrombin tested, $1 \mu \mathrm{M}$ resulted in the highest fluorescence intensity, and this followed a trend down to $100 \mathrm{pM}$ thrombin which saw a fluorescence intensity approximately equal to the output from the aptamer in the absence of thrombin. 


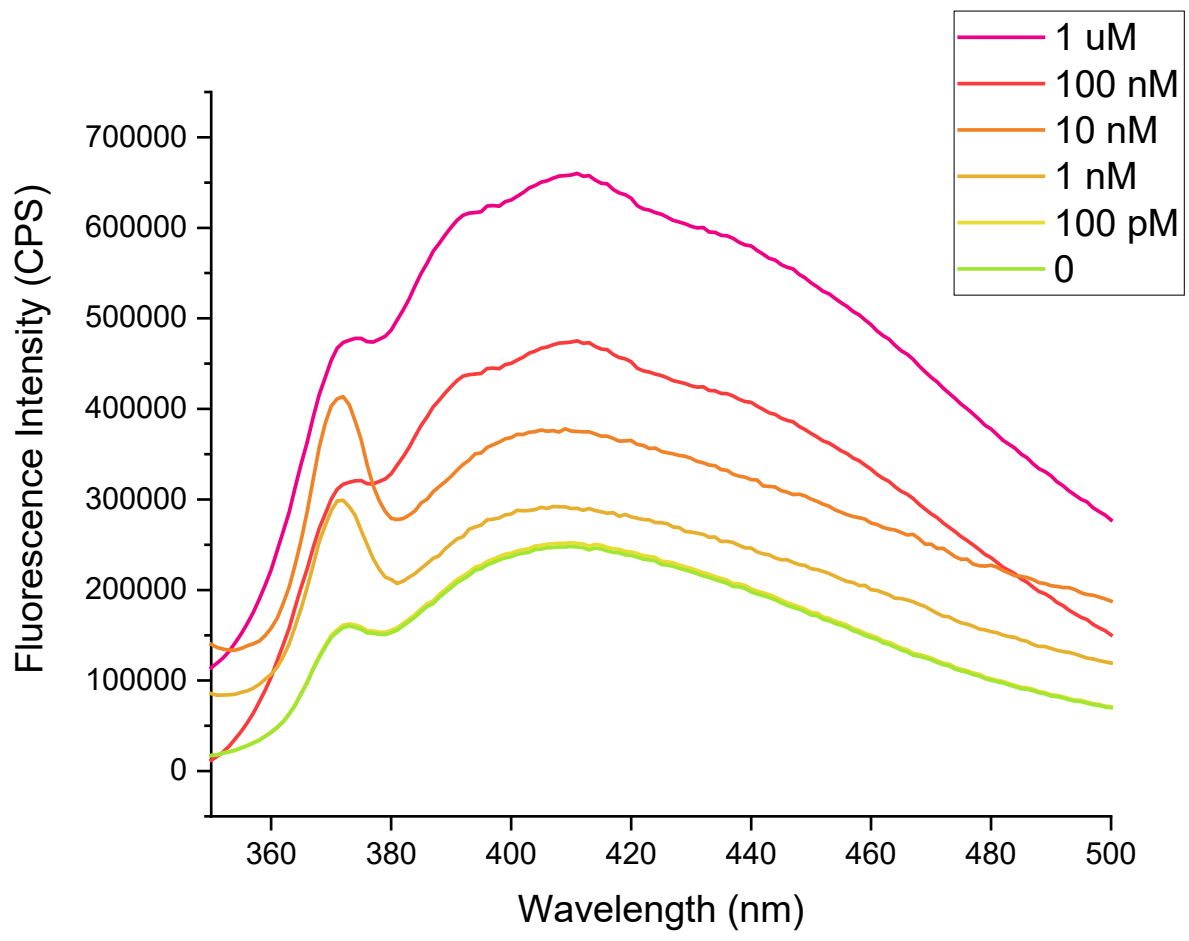

Figure 4.15: Fluoresence emission spectra measured between $350-500 \mathrm{~nm}$ with an excitation of $333 \mathbf{~ n m}$. Reactions consisted of a mixture of the double modified SRA 2 aptamer mixed with its capture probe and the indicated concentration of human $\alpha$-thrombin. No thrombin (0) resulted in the lowest fluorescence emission at $410 \mathrm{~nm}$, with increasing concentrations of thrombin resulting in a positive increase in fluorescence emission.

Summarizing this data in a bar plot as in Figure 4.16 demonstrates a roughly linear trend between the fluorescence emission intensity at $410 \mathrm{~nm}$ and the concentration of thrombin. The sensor appears to plateau to the same value as the background in the absence of thrombin once the thrombin concentration reaches $100 \mathrm{pM}$. Each of these reactions was only run a single time, and therefore error bars are not included. For a more complete investigation more starting material would be required to increase the replicates of each reaction and explore a wider range of thrombin concentrations to more accurately judge the sensor. Following the same examples of thrombin aptasensors developed when 
comparing the thrombin tripartite system to literature, this system does present a simplified system for the rapid detection of cocaine in a signal-on system with a high level of sensitivity for thrombin that rivals most reported thrombin aptasensors. Further research will be required to collect sufficient replicates to establish the linear dynamic range and limit of detection of the system, but at the moment it appears as though a linear relationship exists over the $1 \mu \mathrm{M}$ to $1 \mathrm{nM}$ thrombin concentration range with a limit of detection in the picomolar range. This would be comparable to the tripartite system data but presents a simplified system once the aptamer is synthesized with sufficient yields.

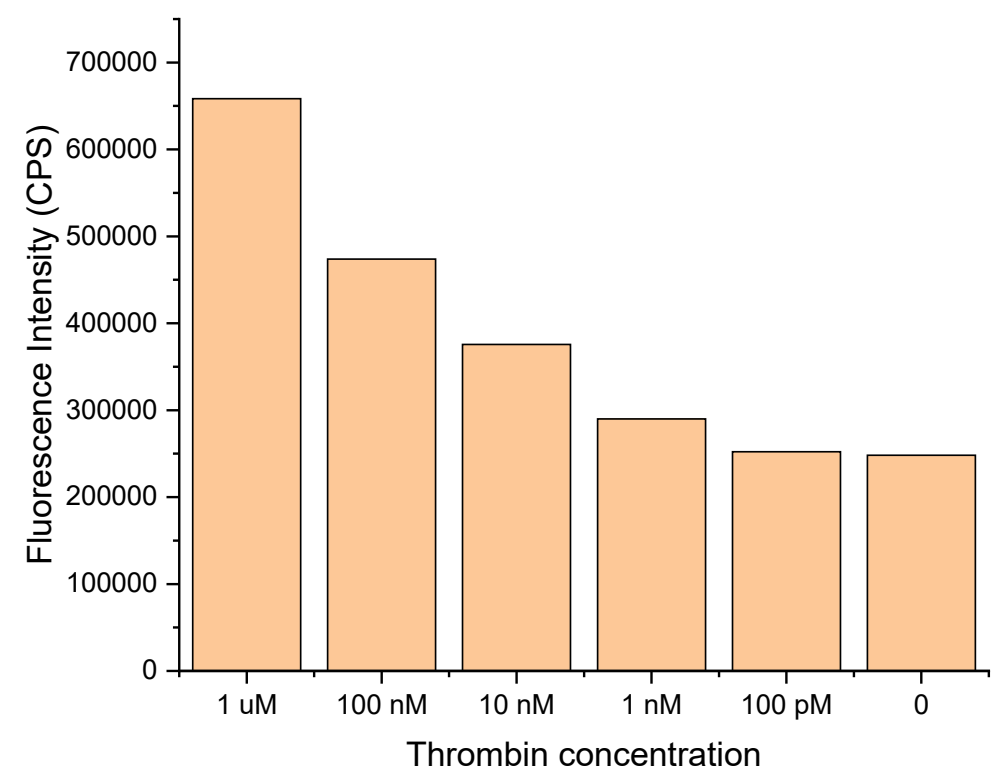

Figure 4.16: Bar plot conversion of the data from Figure 4.15 showing the measured fluorescence intensity at $410 \mathrm{~nm}$ for each of the reactions with the indicated concentration of thrombin. No thrombin (0) resulted in the lowest fluorescence emission at $410 \mathrm{~nm}$, with increasing concentrations of thrombin resulting in a positive increase in fluorescence emission. Data was collected with a single replicate. 


\subsubsection{Linear dynamic range of single aptamer thrombin sensor system}

To evaluate the utility of a sensor the final analysis that must be done is an evaluation of the linear dynamic range and the limit of detection. Seeing as only a single replicate of each reaction was performed it is not possible to evaluate the limit of detection, but an approximation of the linear dynamic range can be made based on the data provided. By omitting the $100 \mathrm{pM}$ thrombin concentration at which the plateau was observed, a roughly linear positive relationship is observed between the concentration of thrombin and the fluorescence emission intensity at $410 \mathrm{~nm}$ in Figure 4.17. The linear dynamic range for this sensor based on the experiments performed can be estimated as $1 \mu \mathrm{M}$ to $1 \mathrm{nM}$ thrombin concentration.

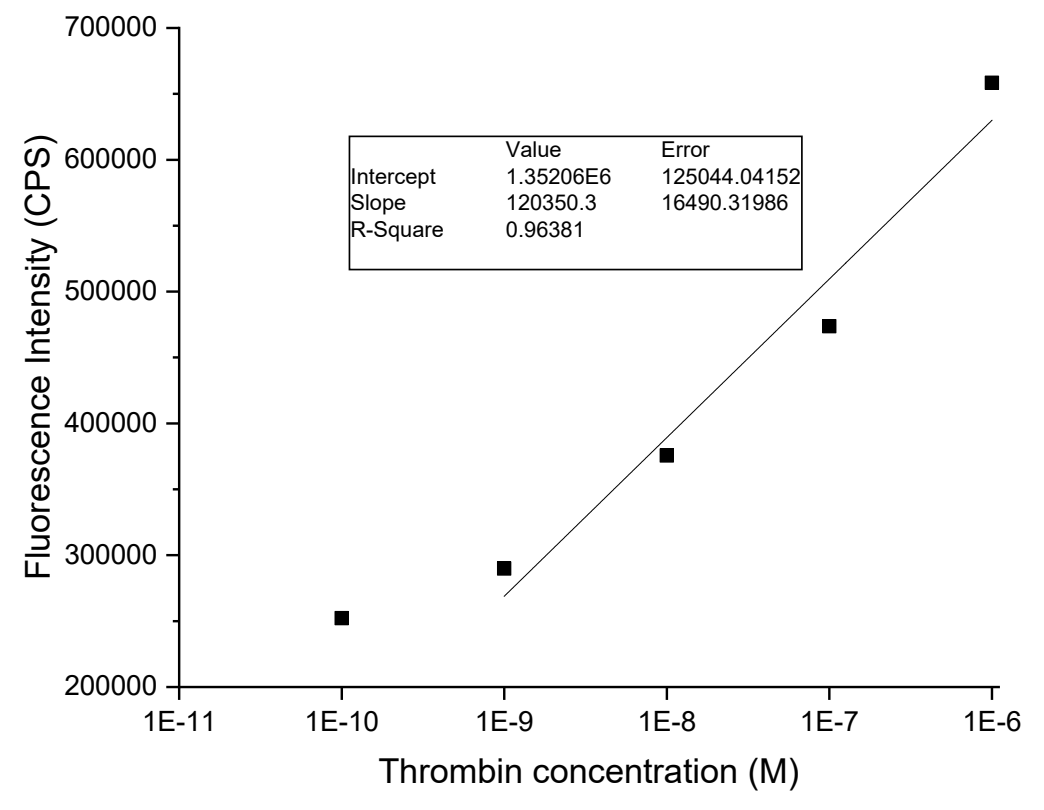

Figure 4.17: Scatter plot of the observed trend for the reaction in the presence of $1 \mu \mathrm{M}$ to $1 \mathrm{nM}$ human $\alpha$-thrombin concentrations. Data was collected from a single replicate. 


\subsection{Conclusions}

The development of two different styles of thrombin sensors based on fluorogenic oligonucleotide templated or oligonucleotide enabled reactions was demonstrated. The tripartite thrombin fluorogenic reaction between an azide and alkyne-coumarin probe complementary to the SRA_2 thrombin aptamer demonstrated the potential for the development of a signal off sensor. Using this system, a linear dynamic range of $100 \mathrm{nM}$ to $10 \mathrm{pM}$ thrombin concentrations was seen, and a limit of detection of $3.9 \mathrm{fM}$ was calculated. This is a relevant sensor for downstream applications with thrombin based on this linear dynamic range being within the range of thrombin expected in the blood during a coagulation event. For the single aptamer system, rational incorporation of the fluorogenic azide and alkyne modifiers within the sequence such that the complementary capture strand inhibits their reaction until the thrombin target binding event results in the moieties being brought into proximity proved successful. Upon incorporation of $1 \mu \mathrm{M}$ to $1 \mathrm{nM}$ thrombin concentrations a linear trend was observed, demonstrating the utility of this turn on sensor system. Studies of the thrombin single sensor aptamer system were limited by poor yields, but further exploration of the reactions involved in synthesizing this double modified aptamer should result in a greater yield and a greater understanding of the single aptamer sensor system.

In addition to the positive results demonstrating that a functional thrombin sensor was designed using two different strategies, it is also important to note that both the tripartite signal off sensor system and the single aptamer signal on sensor system could be applied to a variety of systems where a reaction between two reactive elements needs to be 
controlled. While a similar trend was observed for the QUAL reaction in chapter 3, this system demonstrates that background reactions can be eliminated through the incorporation of more stable modifiers. Systems based on either the single aptamer or tripartite aptamer sensor could be designed for a variety of applications where a reaction needs to be controlled- not just for sensors. Downstream applications related to spatiotemporal control of a reaction for applications in medicine and agricultural industries could be envisioned.

It should also be noted that the click ligation chemistry implemented in this section also constitutes a proof of concept using known reactive elements. In the future, designing novel chemistry could open new avenues, such as the design of copper-free reactions that rely on ring strain in the alkyne to drive the reaction. Other applications would involve making the system chromogenic, for the generation of the first controllable chromogenic reactions for an easier readout of the reaction test in the field for the development of simple sensors. 


\section{Chapter: The detection of cocaine using a split aptamer modified with fluorogenic click handles}

\subsection{Statement of contributions}

Christopher Mattice performed all experimental design, modifier and DNA syntheses, and experimentation related to the cocaine split aptamer sensor. Transmission electron microscopy (TEM) studies were performed at the Nano Imaging Facility at Carleton University by Jianqun Wang.

\subsection{Introduction}

\subsubsection{Split aptamers}

Biosensors can generally be classified as either sandwich or competitive assays depending on the number of recognition elements involved. Sandwich assays require two recognition elements to come together for analyte detection, and are considered superior in terms of specificity and sensitivity as a result of the dual recognition system. ${ }^{186}$ Sandwich assays for small molecules are more difficult than for large macromolecules, as two separate recognition epitopes on the molecule are unlikely and therefore steric hindrance results in competition for binding between the two recognition elements. ${ }^{186}$

To mitigate this issue, split-aptamer based strategies were developed by Stojanovic et al. in 2000. Oligonucleotide aptamers generally consist of a single oligonucleotide sequence with two partially complementary domains, which are connected through 
loops. ${ }^{187}$ This structure is referred to as a hairpin loop. At the time it was surmised that the hairpin loop generally is not involved in the binding event between the aptamer and its target, and could therefore be removed to generate two separate aptamer subunits. ${ }^{187}$ These two separate subunits are termed split aptamers, and rely on ligand-dependent selfassembly of the two fragments much like heavy and light chain antibody fragments. In the case of a sandwich assay, each of the split aptamer sequences could be labeled with reactive groups or a FRET pair to produce a positive signal upon target binding, which brings the two split aptamer sequences together. ${ }^{186}$

The initial experiments by Stojanovic et al. demonstrated that this strategy could be used to produce split aptamers from known aptamer sequences such as the cocaine binding aptamer. ${ }^{187}$ To demonstrate a wider range of applicability it was also demonstrated in the same paper that a split aptamer for ribonucleotide adenosine triphosphate (rATP) could be produced using the same loop cleavage strategy. ${ }^{187}$ Due to the complexity associated with determining aptamer tertiary structure, very few split aptamers currently exist in literature. Aptamers with well-defined aptamer-target complex structures such as the theophylline aptamer are well suited to produce split aptamers as the split site can be well defined without disturbing target binding affinity. Hairpin loops can also be readily used as cleavage sites as predicted by Stojanovic, but these need to be sufficiently distant from the target binding site to not perturb affinity. This was used by Liu et al. to produce a split aptamer for $17 \beta$-estradiol. ${ }^{188}$ A summary of currently published split aptamers can be found in table 1 . 
Table 5.1: A summary of currently published DNA and RNA split aptamers

\begin{tabular}{|c|c|c|}
\hline Target & Fragment 1 & Fragment 2 \\
\hline $\mathbf{A T P}^{187}$ & ACCTGGGGGAGTAT & TGCGGAGGAAGGT \\
\hline Cocaine $^{187}$ & GTTCTTCAATGAAGTGGGACGACA & GGGAGTCAAGAAC \\
\hline Thrombin $^{189}$ & GGTTGGTG & TGGTTGG \\
\hline 17及-estradiol ${ }^{188}$ & $\begin{array}{c}\text { GCTTCCAGCTTATTGAATTACACGC } \\
\text { AGAGGGTA }\end{array}$ & $\begin{array}{c}\text { GCGGCTCTGCGCATTCAATTGCTGCG } \\
\text { C-GCTGAAGCGCGGAAGC }\end{array}$ \\
\hline D-vasopressin 190 & TCACGTGCATGATAGACGGCG & $\begin{array}{l}\text { AAGCCGTCGAGTTGCTGTGTGC- } \\
\text { CGATGCACGTGA }\end{array}$ \\
\hline Theophylline ${ }^{191}$ & $\begin{array}{c}\text { rGrGrCrGrArUrArCrCrArGrCrCrGrArA } \\
\text { rA }\end{array}$ & rGrGrCrCrCrUrUrGrGrCrArGrCrGrUrC \\
\hline ZY11 cells ${ }^{192}$ & $\begin{array}{l}\text { CGTCAGGTTGAGCTGAAGATCGTA } \\
\text { CCGTGAAGTCCGT }\end{array}$ & ACGGACTACCTGGCG \\
\hline CCRF-CEM cells ${ }^{193}$ & $\begin{array}{c}\text { TACTGTACGGTTAGATCTGCCTGCT } \\
\text { CATCTAACTG } \\
\text { CTGCGCCGCCGGGAAAA }\end{array}$ & GAGCAGGCAG \\
\hline Lysozyme $^{194}$ & $\begin{array}{l}\text { TGGGTTACAGTGCAGAGTTACTTA } \\
\text { G }\end{array}$ & $\begin{array}{c}\text { ATCAGGGCTAAAGAGCTGTAGGGTA } \\
\text { GGGCGGG }\end{array}$ \\
\hline
\end{tabular}

Split aptamers have seen many applications in the production of electrochemical and surface-plasmon resonance-based sensors. One such example from Lin et al. designed an electrochemiluminescence sensor for thrombin by modifying one aptamer sequence with thiol to allow for immobilization on the gold electrode while the other sequence was modified with $\mathrm{Ru}(\mathrm{bpy}){ }_{3}{ }^{2+}$ doped silica nanoparticles. This resulted in a limit of detection of $3.3 \mathrm{pM}$ with a high level of specificity. ${ }^{195}$ A similar strategy was undertaken by Chen et al., substituting a ferrocene tag on one of the sequences to produce a readily detectable fragment in the presence of thrombin protein. ${ }^{196}$ Wang et al. demonstrated the first split aptamer based SPR sensor in 2011 for the detection of ATP in solution. In this strategy, one of the split aptamer sequences is attached to a gold film, while the other sequence is attached to gold nanoparticles, resulting in a $1.5 \mathrm{pM}$ detection limit. ${ }^{197}$ A low-affinity reversible split aptamer sensor system for ATP was also demonstrated by Sergelen et al., which allowed for sensitive detection along with the continuous monitoring of analytes. 
The low affinity aptamer allows for rapid regeneration of the system for continuous sample analysis. $^{198}$

Split aptamers have also been used to catalyze DNA ligations and related DNA catalyzed reactions. Heemstra et al. demonstrated in 2011 that a strain promoted azidealkyne cycloaddition reaction could be initiated between two cocaine split aptamers through the addition of cocaine. ${ }^{149}$ Heemstra also demonstrated Split Aptamer Proximity Ligation (StAPL) in 2012, which is the ligation of split aptamer fragments through end labeling with reactive groups to translate a small molecule signal into a DNA ligation event. ${ }^{199}$ In order to convert this ligation into a chromogenic event, one of the aptamer sequences was coupled to horseradish peroxidase after washing. ${ }^{199}$ Sun et al. also demonstrated the initiation of an external fluorogenic reaction between 3-azidocoumarin and an alkyne, catalyzed by the release of copper sulfide nanoparticles (CuS NPs) associated with the unbound split aptamers upon ATP target binding. ${ }^{200}$

Another common sensor application for split aptamers are colourimetric and FRET based systems. Split aptamers have been used to produce colourimetric logic gates with both AND and OR functionality using gold nanoparticles and two different split aptamers. For the OR logic gate, each of the split aptamer sequences for cocaine and ATP are ligated into a single sequence to produce a mixed split aptamer. The tight association of DNA with gold nanoparticles inhibits their aggregation upon addition of salt, unless either of ATP or cocaine or both are added to the system, which would result in the mixed split aptamers binding to the target and the gold nanoparticles aggregating. The AND gates are produced using a three-sequence system, in which only one side of the split aptamers are ligated. For 
all three sequences to bind to the target and leave the gold nanoparticle unprotected, both targets are required, resulting in an AND logic gate. ${ }^{201}$ Liu et al. took advantage of conjugated polymers and their delocalized electronic structure to quench the fluorescence of one labeled sequence of a split aptamer. Upon addition of the thrombin target, steric hindrance resulted in a greater distance between the conjugated polymer and the fluorophore on the bound aptamer, resulting in a FRET signal decrease. ${ }^{202}$ FRET between Cy3 and Cy5 dyes was also used to develop a sensor using the first split aptamer for a tumour cell, ZY11. ${ }^{192}$

There are also split aptamer applications that are not solely related to sensor development. One such example is the development of theranostic agents for cancer cell imaging and drug delivery as demonstrated by Lei et al. In this application, a split aptamer against human leukemia CCRF-CEM cells was developed and demonstrated to allow for specific labeling of cancer cells using fluorophores as well as the specific delivery of doxorubicin to those cancer cells. ${ }^{193}$ Split aptamers have also been developed for the detection of latent fingerprints by Liu et al. This split aptamer was selected against the enzyme lysozyme to generate a G-quadruplex upon target binding. Formation of the Gquadruplex could be detected through the addition of $N$-methyl mesoporphyrin IX (NMM), a dye that is selective to G-quadruplexes. ${ }^{194}$

\subsubsection{Cocaine split aptamer}

The cocaine binding aptamer was first selected and published over a series of papers by Stojanovic et al. between 2000 and 2002. ${ }^{187,203-204}$ The designed aptamer following 
optimization, MNS-4.1, is a 39-mer sequence consisting of three loops with a central threeway junction where cocaine binds and is shown in the originally proposed structure in Figure 5.1a. ${ }^{205}$ Stem 2 contains only Watson-Crick base pairs, while stem 1 and stem 3 contain two non-canonical base pairs each. ${ }^{205}$ The affinity of MNS-4. 1 for the cocaine target is on the order of 5-10 $\mu \mathrm{M}$ depending on the method of analysis, with Neves et al. reporting an affinity of $9.15 \pm 0.09 \mu \mathrm{M} .^{205-206}$ NMR analysis by the same group lead to the proposal of tandem GA base pairs in the structure with a two-nucleotide bulge where cocaine binding occurs (Figure 5.1b). ${ }^{206}$ The binding mechanism of the full-length 6-base stem 1 version of the cocaine binding aptamer shown suggests that the secondary structure of the aptamer is pre-formed in solution, and that ligand binding induces very little change in secondary structure. ${ }^{205}$ The cocaine aptamer also shows an abnormally higher binding affinity for quinine than for cocaine, a target that it was not selected against. ${ }^{207}$
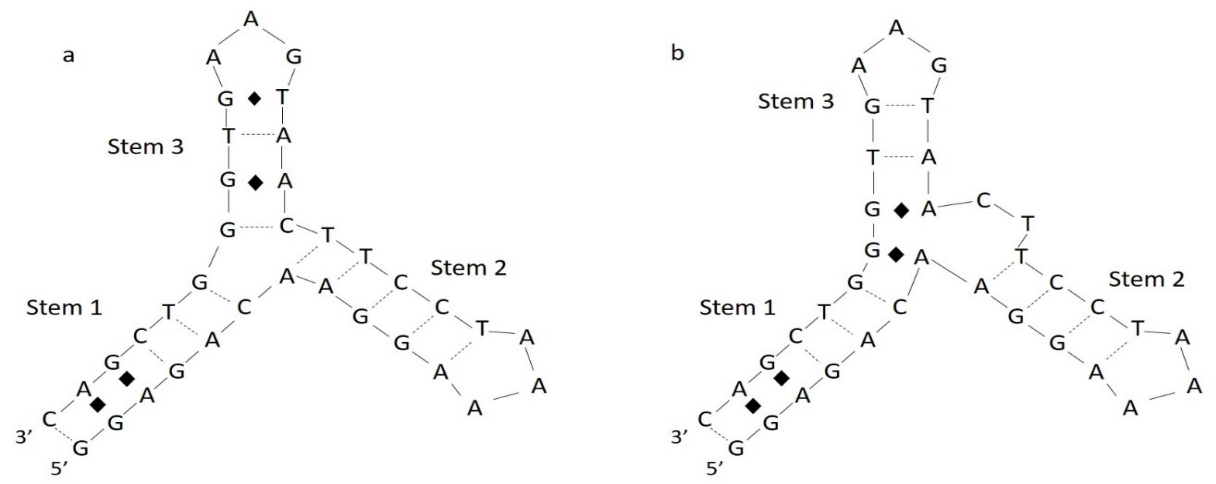

Figure 5.1: a) Originally proposed structure of the cocaine aptamer MNS-4.1 by Stojanovic et al. b) Improved NMR elucidated cocaine aptamer MNS-4.1 structure by Neves et al. showing the tandem GA mismatch and the two nucleotide bulge in the cocaine binding pocket. ${ }^{187,203,206}$

After the development of the cocaine split aptamer by Stojanovic et al. in 2000, it quickly became one of the most studied split aptamers alongside the ATP aptamer. The 
initial selection started from the known 39-mer cocaine aptamer with a reported $K_{d}$ of 5 $\mu \mathrm{M}$, and was split in stem 2 to produce two subunits with an apparent $\mathrm{K}_{\mathrm{d}}$ of $200 \mu \mathrm{M} .{ }^{187}$ The structure of the cocaine split-aptamer on target binding is believed to be similar to that shown for the modified MNS4.1 aptamer demonstrated by Neves et al. ${ }^{206}$ The first cocaine split aptamer sensor consisted of a fluorophore-quencher pair labeled on each terminus of the split aptamer fragments to demonstrated fluorescence quenching upon cocaine target binding. ${ }^{187}$

A wide range of cocaine split aptamer sensors have been developed since 2011, some of which will be briefly touched on herein. Zhang et al. developed a cocaine sensor based on the cocaine split aptamer and gold nanoparticles. Aggregation of the gold nanoparticles resulted in a colour shift upon addition of the cocaine target. This resulted in a visible limit of detection of $2 \mu \mathrm{M}$, which has the advantage of not requiring any specialized equipment to obtain a qualitative readout. ${ }^{208}$ Electrochemiluminescent sensors have also been produced for the cocaine split aptamer to detect the presence of cocaine on banknotes. This system could quantify cocaine with a limit of detection of $3.7 \mathrm{pM}$, and a linear dynamic range of $1 \mathrm{nM}$ to $10 \mathrm{pM}^{209}$ An aptasensor for cocaine based on the evanescent wave optical biosensing platform was demonstrated recently by Tang et al. This system allowed for the rapid detection of cocaine down to $200 \mu \mathrm{M}$ with near instant surface regeneration for continuous sample flow. ${ }^{210}$

Another recent development in cocaine split aptamer sensors has been the synthesis of cooperative-binding split aptamers for cocaine, which permitted the fluorescence detection of cocaine in saliva by $\mathrm{Yu}$ et al. ${ }^{211}$ This group sought to enhance aptamer affinity 
over the standard cocaine split aptamer, since split aptamers are known to have a reduced target affinity relative to their parent aptamer. Split aptamers suffer from significant background signal on most occasions simply due to their folding activity in the absence of target due to their inherent complementarity. This background can be avoided by splitting the aptamer into smaller segments, but this further reduces the affinity. ${ }^{187,211} \mathrm{Yu}$ et al designed aptamers with two tandem target-binding domains within the split aptamer, a system they named cooperative binding split aptamer, CBSA. In this system, the initial cocaine binding event stabilizes the structure of the split aptamer and facilitates the binding of a second molecule of cocaine. This system could attain a limit of detection of $50 \mathrm{nM}$ cocaine in buffer and saliva in 15 minutes. ${ }^{211-212}$

\subsubsection{Cocaine}

Cocaine is an alkaloid drug produced by the plant Erthroxylum coca, found primarily in the northern parts of South America. Cocaine in its pure form is a white powder and is the hydrochloride salt which is consumed through nasal insufflation (snorting). Crack cocaine on the other hand is the free base, and is a yellow solid that is ingested through smoking. ${ }^{213}(R)-(-)$-cocaine is the only addictive configuration of the eight possible stereoconformers of cocaine. ${ }^{214}$

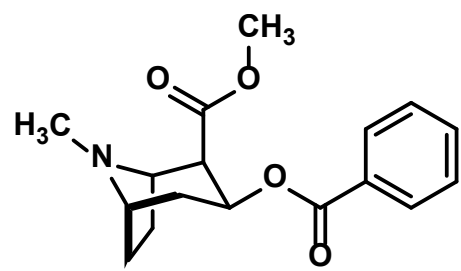




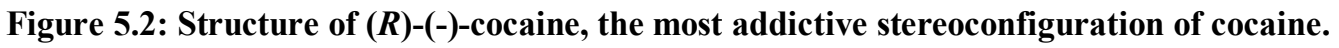

Native South Americans have consumed coca tea or chewed the leaves of coca plants, releasing cocaine into their saliva for thousands of years. Reported benefits include stimulation and rejuvenation, as well as the alleviation of hunger and thirst. ${ }^{215}$ It is also commonly used by travelers to South America for the relief of altitude sickness, especially in and around Machu Picchu. ${ }^{213,215}$ Cocaine was first isolated by Niemann in 1855, and popularized by Sigmund Freud for its stimulant and euphoric effects in $1884 .^{213}$ Cocaine was not banned as a drug in the United States until 1914, though its addictive and damaging properties had been acknowledged for decades. In the 1970s abuse of cocaine increased significantly, with the infamous cartel kingpin Pablo Escobar monopolizing the import and sale of cocaine into the United States. As of 2002, 1.9 million people in the United States identified as cocaine users, compared to 3.8 million opioid users and 4 million cannabis users. $^{213}$ Despite the comparatively low use, cocaine is still the most highly intercepted drug globally and the most profitable. Manufacturing one kilogram of pure cocaine costs the cartel an average of $\$ 10,000$, with the final markup on selling yielding $\$ 150,000$ on average when split into 1-gram quantities selling for $\$ 150$ depending on the region. ${ }^{213}$

Cocaine has many pharmacological roles, including its role as a local anesthetic which was discovered by Carl Koller in $1884 .{ }^{216-217}$ This is due to cocaine's inhibition of the voltage-gated sodium channels, thereby inhibiting neuronal firing. ${ }^{218}$ Cocaine also acts as a psychomotor stimulant through inhibition of dopamine and norepinephrine reuptake transporters. ${ }^{219}$ Cocaine is also cardiotoxic, as it results in prolonged vasoconstriction thereby decreasing blood flow to the heart and causing myocardial infarction in prolonged 
used cases. $^{218,220}$

\subsubsection{Proposed fluorogenic split aptamer ligation sensor}

The goal of this project was to develop the first fluorogenic DNA ligation click reaction using the cocaine split aptamer. Though Heemstra had previously demonstrated the detection of cocaine through Split Aptamer Proximity Ligation (StAPL) with strain promoted azide-alkyne cycloadditions as well as enzyme-linked assays, both strategies were cumbersome with additional reagents required. The strategy for detecting cocaine used in this project used the alkyne-coumarin and azide DNA modifiers prepared previously and end labeled the two fragments of the cocaine split aptamer reported by Stojanovic et al. ${ }^{187}$ In the absence of cocaine, but in the presence of a copper(I) source, no background reaction should be observed as the split aptamers will not be able to come into close proximity at the concentration tested. In the presence of cocaine, the two modifiers will be brought into proximity, allowing the copper(I) catalyzed azide-alkyne cycloaddition to occur, thereby ligating the two DNA strands.

The advantage of such a system over the previous ligating DNA reactions is the immediate fluorescent readout after the reaction. The strain promoted azide-alkyne cycloaddition reaction suffers from the reaction outcome only being observable through gel electrophoresis or HPLC. Coupling the system to the horseradish peroxidase enzyme does generate a signal readout, but this requires additional steps after the ligation reaction. This system also has advantages over the other strategies that we have undertaken for developing OTR sensors, in that this simple system allows for a turn-on sensor without 
complicated reactivity to incorporate modifiers within the sequence. ${ }^{149,} 199$ If cocaine is present, the strands will ligate to produce a signal. In the absence of cocaine there will be no signal. This results in a much more sensitive system due to the low background signal.

A

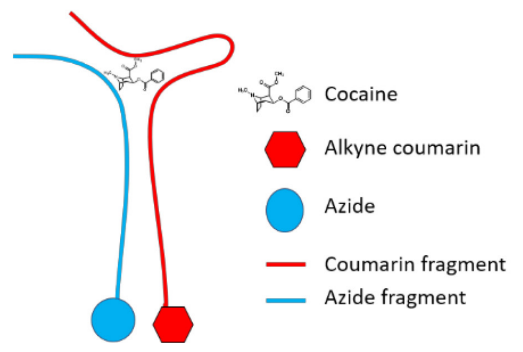

B

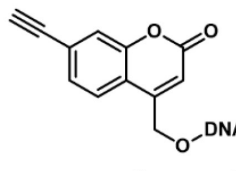

non fluorescent

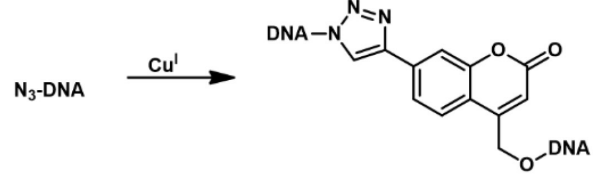

fluorescent

Figure 5.3: A) A sensor for cocaine can be designed by labeling the two fragments of a cocaine split aptamer with a fluorogenic azide-alkyne pair that will only be able to react in the presence of cocaine. B) The fluorogenic azide-alkyne cycloaddition occurs between an alkyne-coumarin profluorophore and an azide to generate a fluorescent coumarin-triazole.

\subsection{Experimental}

\subsubsection{Materials and equipment}

All oligonucleotide sequences were synthesized using a BioAutomation MerMade 6 oligonucleotide synthesizer using the solid phase phosphoramidite method. Azidobutryate NHS ester, 3'-amino modifier C7 CPG, Pac-dA-CE phosphoramidite, iPrPac-dG-CE phosphoramidite, 5\% phenoxyacetic anhydride in THF Cap Mix A, and 0.05M potassium carbonate in methanol were purchased from Glen Research. All necessary reagents for DNA synthesis including standard phosphoramidites, activator, deblock, oxidizer, capping agents, and activator were purchased from Glen Research. Standard controlled pore glass (CPG) columns were purchased from Bioautomation. Biosynthesis grade acetonitrile was purchased from EMD Millipore for use on the DNA synthesizer. 
Ultra-high purity (UHP) argon was purchased from Praxair for use on the DNA synthesizer. Glen Pak DNA purification cartridges were purchased from Glen Research. All buffers were prepared using EMD Millipore Milli-Q deionized water, and buffer salts were purchased from Sigma Aldrich and BioShop Canada Inc. Buffers were filtered through Corning $0.22 \mu \mathrm{m}$ PES vacuum filtration systems from Sigma Aldrich. Acrylamide/bis-acrylamide $40 \%$ solution, electrophoresis grade ammonium persulfate (APS), $N, N, N^{\prime}, N^{\prime}$-tetramethylethylenediamine (TEMED), ethylenediaminetetracetic acid (EDTA), boric acid, tris(hydroxymethyl) aminomethane (Tris), and urea were purchased from Bioshop Canada Inc. Cocaine hydrochloride, copper(II) sulfate pentahydrate, and $(+)$-sodium-L-ascorbate were purchased from Sigma Aldrich. UV/Visible absorption spectra were acquired using a Varian Cary 300 Bio/UV visible spectrophotometer. Fluorescence spectra were acquired using a Horiba FluoroLog-3.

\subsubsection{Buffer}

Table 5.2: Buffer composition for ochratoxin A quenched autoligation probe sensor experiments.

\begin{tabular}{c|c|c} 
Buffer name & Composition & pH \\
\hline Cocaine buffer & $25 \mathrm{mM} \mathrm{Tris}$ & 8.2
\end{tabular}




\subsubsection{DNA synthesis, purification, and quantification}

Table 5.3: Oligonucleotide sequences used in the fluorogenic split aptamer click experiment for designing a cocaine sensor. The abbreviation "coum" is used to indicate the location of the alkynecoumarin DNA modifier, while the $\mathrm{N}_{3}$ label is used to indicate the location of the azide modifier.

\begin{tabular}{c|c} 
Oligonucleotide & Sequence \\
\hline $\begin{array}{c}\text { Cocaine coumarin } \\
\text { Cocaine azide }\end{array}$ & 5'-Coum-GTT-CTT-CAA-TGA-AGT- \\
GGG-ACG-ACA-3' \\
5'-GGGAGTCAAGAAC-N ${ }_{3}-3$
\end{tabular}

The cocaine coumarin was prepared under ultramild DNA synthesis conditions on the Bioautomation Mermade 6 DNA synthesizer. $1 \mu$ mol 5'-DMT-dA $1000 \AA ̊$ columns were used for the synthesis. The machine was switched to use ultramild reagents including Pac-dA-CE phosphoramidite for all adenine bases, ipr-Pac-dG-CE for all guanine bases, and ultramild Cap Mix A (5\% phenoxyacetic anhydride in THF) in place of the standard Cap Mix A. The alkyne-coumarin phosphoramidite from chapter 3 was prepared fresh on the day of DNA synthesis and resuspended in acetonitrile at $0.067 \mathrm{M}$ concentration, and placed in one of the modifier positions on the synthesizer. The coupling time for the alkyne-coumarin modifier was extended to 10 minutes, and the final deprotection step was omitted based on the location of the alkyne-coumarin in the DNA sequence. For the azide probe, this was first synthesized as an amine for subsequent incorporation of the azide modifier. Using 3'-amino modifier C7 CPG $1000 \AA$ columns, the amino modified variant of the cocaine azide probe was synthesized under normal DNA synthesis conditions according to manufacturer recommendations. The final deprotection step was omitted to maintain the final DMT modifier for subsequent processing on the Glen Pak.

Upon synthesis completion, the synthesis columns for both probes were removed 
from the DNA synthesizer, and the contained CPG support beads were transferred into a microcentrifuge tube. For the azide probe (currently amino modified) the CPG beads were deprotected in $1 \mathrm{~mL}$ of $28 \% \mathrm{NH}_{4} \mathrm{OH}$ for 16 hours at $55^{\circ} \mathrm{C}$, before proceeding to Glen Pak purification. For the coumarin modified cocaine probe, the CPG beads were deprotected for 4 hours at room temperature in $0.05 \mathrm{M} \mathrm{K}_{2} \mathrm{CO}_{3}$ in methanol before proceeding to Glen Pak desalting.

\subsubsection{Glen Pak DNA purification}

The amine modified azide probe was subjected to standard Glen Pak purification on a Glen Pak cartridge. The CPG deprotection solution in $28 \% \mathrm{NH}_{4} \mathrm{OH}$ was decanted into a new microcentrifuge tube and the beads were washed with $100 \mathrm{mg} / \mathrm{mL} \mathrm{NaCl}$ in deionized water, before decanting and mixing with the initial deprotection solution. One Glen Pak cartridge for each of the six synthesis columns was loaded onto a Phenomenex 12 port vacuum manifold with an attached oil-free vacuum pump and rinsed with $0.5 \mathrm{~mL}$ of acetonitrile followed by $1 \mathrm{~mL}$ of $2.0 \mathrm{M}$ triethylammonium acetate (TEAA). The ammonium hydroxide/ $\mathrm{NaCl}$ deprotection solution was loaded onto each column in turn 1 $\mathrm{mL}$ at a time and then rinsed twice with $1 \mathrm{~mL}$ of $5 \%$ acetonitrile in $100 \mathrm{mg} / \mathrm{mL}$ sodium chloride. The columns were then deprotected with two $1 \mathrm{~mL}$ aliquots of freshly prepared $2 \%$ trifluoroacetic acid in deionized water, at which point a bright orange band was observed. Rinsing each column twice with $1 \mathrm{~mL}$ of deionized water removed the orange bad. The collection tubes under the vacuum manifold were then switched, and the target sequence eluted using a $1 \mathrm{~mL}$ solution of $50 \%$ acetonitrile in deionized water with $0.5 \%$ 
ammonium hydroxide. The eluted samples were then dried on a Savant AES2010 SpeedVac cryopump overnight before resuspension in deionized water and quantification.

For the coumarin cocaine probe, a Glen-Pak desalting protocol was followed, wherein the potassium carbonate in methanol deprotection solution was diluted to $20 \mathrm{~mL}$ in 0.1 M TEAA. The cartridges were prepared as before, and the diluted deprotection solution was added to each of the columns. After loading, each of the columns was rinsed twice with $1 \mathrm{~mL}$ of deionized water. The collection tubes were switched for each of the columns, and the cocaine coumarin probe eluted in $1 \mathrm{~mL}$ of $50 \%$ acetonitrile in deionized water. The eluted samples were then dried on a Savant AES2010 SpeedVac cryopump overnight before resuspension in deionized water and preparation for polyacrylamide gel electrophoresis.

\subsubsection{Incorporation of the azide modifier}

The azide was incorporated onto the amine labeled cocaine probe using azidobutryate NHS-ester. The azidobutyrate NHS-ester was dissolved in $60 \mu \mathrm{L}$ of DMSO to a concentration of $0.17 \mathrm{M}$. $500 \mathrm{nmol}$ of the amine modified sequence was dissolved in $250 \mu \mathrm{L}$ of $0.5 \mathrm{M} \mathrm{Na}_{2} \mathrm{CO}_{3} / \mathrm{NaHCO}_{3}$ buffer $\mathrm{pH}$ 8.75. All $60 \mu \mathrm{L}$ of the azidobutyrate NHSester solution were added to the amino-modified sequence in buffer, and the reaction was run for 4 hours at room temperature. The reaction was stopped through the addition of 4 $\mathrm{mL}$ of $0.1 \mathrm{M}$ TEAA, and then desalted according to the previously describe Glen Pak desalting protocol. 


\subsubsection{Polyacrylamide gel electrophoresis}

The cocaine coumarin probe was purified using a 19\% denaturing polyacrylamide gel. The gels were prepared by mixing $63 \mathrm{~g}$. of urea with $75 \mathrm{~mL}$ of acrylamide solution (acrylamide/bisacrylamide $40 \%$ solution), and $30 \mathrm{~mL}$ of $5 \times$ TBE buffer (45 mM Tris, 45 $\mathrm{mM}$ boric acid, $1 \mathrm{mM}$ EDTA). The solution was stirred while heating to $37^{\circ} \mathrm{C}$ and filtered while hot through Whatman No. 1 filter paper. Once cooled to room temperature, $900 \mu \mathrm{L}$ of $10 \%$ ammonium persulfate and $70 \mu \mathrm{L}$ of TEMED were added to the stirred solution. The gel solution was then poured into the casting apparatus for the Hoefer SE600 Chroma standard dual-cooled gel electrophoresis assembly. The gels were pre-run for 20 minutes using a constant voltage of 300 volts. During the pre-running, the dried dabsyl probe samples were resuspended in $300 \mu \mathrm{L}$ of deionized water, and then $300 \mu \mathrm{L}$ of formamide was added to each sample. The samples were heated to $55^{\circ} \mathrm{C}$ for 5 minutes before loading into the gel, with each column of synthesized DNA having its own gel. The gels were run for 1.5 hours, before imaging on a $F_{254}$ TLC plate in the Alpha Imager gel cabinet set on the epi-UV $(\lambda=254 \mathrm{~nm})$ and fluorescence settings $\left(\lambda_{\mathrm{ex}}=302 \mathrm{~nm}\right)$. The relevant band for the cocaine coumarin probe was manually excised from the gel, and placed in a $50 \mathrm{~mL}$ Falcon tube. The probe was eluted from the gel using a crush and soak method using deionized water and incubated on a New Brunswick Scientific Innova 40 incubator/shaker at $37^{\circ} \mathrm{C}$ for 48 hours.

After extraction, the solution was filtered through $0.22 \mu \mathrm{m}$, low retention, cellulose acetate syringe filters. The samples were then made up to $20 \mathrm{~mL}$ in $0.1 \mathrm{M}$ TEAA and desalted according to the previously described Glen Pak desalting protocol. 


\subsubsection{DNA quantification}

Both probes were quantified by measuring their optical absorbance at $260 \mathrm{~nm}$ when dissolved in deionized water. By considering the molar extinction coefficients as determined through nearest neighbour analysis and the molar absorptivity of each of the modifiers used, an extinction coefficient can be determined. Using the Beer-Lambert law, the solution concentration of the DNA can be back-calculated. Post quantification, all DNA sequences were submitted for electrospray mass spectrometry for molecular weight confirmation by Novatia LLC. Mass spectrometry was performed using a reverse phase HPLC with direct spray into an LTQ-Orbitrap with deisotoping and deconvolution of the results undertaken using Promass software.

\subsubsection{Sensor evaluation with cocaine}

Initial tests for cocaine split aptamer ligation in the presence of cocaine were performed at $1 \mu \mathrm{M}$ concentration of each probe in the presence of a wide range of cocaine concentrations. These tests involved mixing both sequences at $1 \mu \mathrm{M}$ concentration in the presence of $2 \mathrm{mM} \mathrm{CuSO}_{4}$ and $4 \mathrm{mM}$ sodium ascorbate with cocaine concentrations between $10 \mathrm{nM}$ and $1 \mathrm{mM}$. Cocaine was dissolved in cocaine reaction buffer immediately before the reactions were initiated. The testing protocol entailed first heating each of the probes to $55^{\circ} \mathrm{C}$ then cooling to room temperature. The alkyne-coumarin cocaine probe was then added to the cocaine buffer along with the copper sulfate solution, before the azide cocaine probe was added. The reaction was initiated through the addition of the sodium ascorbate solution and allowed to proceed for 1 hour before quenching with $100 \mu \mathrm{L}$ of 
formamide and heating to $55^{\circ} \mathrm{C}$. Reactions were then evaluated by either gel electrophoresis of fluorescence spectroscopy as described below.

\subsubsection{Desalting test}

To evaluate the effect of salts on the fluorescence spectrum and reaction outcome, a series of desalting protocols were followed post reaction. The first protocol was a standard Glen Pak cartridge desalting protocol performed as previously described. The second protocol was performing an ethanol precipitation following completion of the experiment. Ethanol precipitation proceeded by adding $10 \mu \mathrm{L}$ of $3 \mathrm{M}$ sodium acetate followed by $300 \mu \mathrm{L}$ of $95 \%$ ethanol and incubating at room temperature for 15 minutes. The sample was then centrifuged at $14000 \mathrm{x}$ g for 30 minutes at room temperature, and the supernatant discarded. The pellet was then washed with $70 \%$ ethanol and centrifuged again. The pellet was then brought up in $100 \mu \mathrm{L}$ of deionized water. The final protocol involved the use of an Amicon Ultra $0.5 \mathrm{~mL} 3 \mathrm{~K}$ centrifugal filter units for desalting. The sample was loaded into the filter unit and spun at $14000 \mathrm{xg}$ for 30 minutes, and then washed twice with $100 \mu \mathrm{L}$ of deionized water and spun again. Flipping the filter unit over into a new microcentrifuge with centrifugation at $1000 \mathrm{x} \mathrm{g}$ for 5 minutes yielded the desalted probe that was reconstituted in deionized water.

\subsubsection{Transmission electron microscopy}

To prepare TEM grids, $10 \mu \mathrm{L}$ of the cocaine reaction mixture was deposited on a TEM grid (carbon film on 300 mesh copper grid). The sample was left to sit at room temperature overnight to dry before imaging. TEM imaging was done using a FEI Tecnai 
G2 F20 Transmission Electron Microscope (Hillsboro, USA) equipped with a Gatan ORIUS TEM CCD Camera (Pleasanton, USA). Copper nanoparticle size was measured using AmScope Toupview Camera software.

\subsubsection{Final testing protocol}

Final testing for cocaine split aptamer ligation in the presence of cocaine were performed at $10 \mu \mathrm{M}$ concentration of each probe in the presence of a wide range of cocaine concentrations. This protocol involved the incorporation of the click ligand THPTA, which was mixed with the copper sulfate solution in a 1:1 ratio such that the final concentration in the reaction mixture of each salt is $250 \mu \mathrm{M} \mathrm{CuSO}_{4} / \mathrm{THPTA}$ and $400 \mu \mathrm{M}$ sodium ascorbate. Cocaine was dissolved in cocaine reaction buffer immediately before the reactions were initiated and added to each reaction in a range between $100 \mu \mathrm{M}$ and 10 pM. The testing protocol entailed first heating each of the probes to $55^{\circ} \mathrm{C}$ then cooling to room temperature. The alkyne-coumarin cocaine probe was then added to the cocaine buffer along with the copper sulfate- click ligand solution, before the azide cocaine probe was added. The reaction was initiated through the addition of the sodium ascorbate solution and allowed to proceed for 30 minutes before quenching with $100 \mu \mathrm{L}$ of formamide and heating to $55^{\circ} \mathrm{C}$. Reactions were then evaluated by either gel electrophoresis of fluorescence spectroscopy.

Upon reaction completion, the sample was divided in half, with one half loaded into separate lanes of a $19 \%$ gel. The gel was run for 1 hour at a constant $300 \mathrm{~V}$ and then imaged under both UV and fluorescence modes in the Alpha Imager. The other half of the reaction 
was analyzed by fluorescence spectroscopy on a Fluorolog3. From each reaction, $70 \mu \mathrm{L}$ was loaded into a quartz fluorescence cuvette, and the emission spectrum measured between 350 and $500 \mathrm{~nm}$ with an excitation wavelength of $333 \mathrm{~nm}$ and $3 \mathrm{~nm}$ slit widths.

\subsection{Results and discussion}

\subsubsection{Incorporation of alkyne coumarin into the cocaine split aptamer}

The choice of the aptamer sequence used to design a fluorogenic aptasensor for the detection of cocaine is an important parameter to consider. It is for this reason that a split aptamer was chosen, in addition to its favourable properties for the development of a turn on sensor. Designing a signal-on sensor wherein two reactive modifiers are separate under conditions where the target is absent but will be in proximity upon target binding limits the choice of starting aptamer to either one with a clearly defined target bound structure such as was used in chapter 4 for thrombin, or the use of split aptamer sequences. The cocaine split aptamer presents an opportunity for the exploration of turn on sensors using a fluorogenic reaction, as the split aptamer sequences will only undergo interaction in the presence of the cocaine target.

Using the cocaine split aptamer designated in Table 5.1, a sensor was designed based on the previous work of Heemstra in the strain promoted azide-alkyne cycloaddition with the cocaine split aptamer. ${ }^{149}$ In place of the cyclooctyne modifier from their experiment, we were able to incorporate our alkyne-coumarin profluorophore designed and synthesized in chapter 3. By converting the alkyne-coumarin into a phosphoramidite, it could be directly added onto the DNA synthesizer for incorporation as a modifier. To help 
protect the alkyne-coumarin in the subsequent deprotection step due to its unknown lability, it was decided that an ultramild DNA synthesis strategy would be used. This ultramild synthesis converts the protecting groups on the DNA bases adenine and guanine into more easily removed groups, allowing the use of potassium carbonate in methanol at room temperature for short reaction times instead of $55^{\circ} \mathrm{C} \mathrm{NH} 4 \mathrm{OH}$ overnight. This may have been an unneeded precaution, but the time required to synthesize the alkyne coumarin made it a rather expensive reagent, and the decreased yield of ultramild DNA synthesis way be warranted relative to complete decomposition of the alkyne modifier.

After purification, quantification of the alkyne coumarin resulted in a yield of 500 nmol of modified DNA from six $1 \mu$ mol synthesis columns. While low, this is to be expected for a bulky modifier being incorporated into the sequence and the decreased yields associated with ultramild synthesis methods. It is likely that the short 24-base sequence contributed to higher yields with the competing negative factors. Electrosprayionization mass spectrometry (ESI-MS) molecular weight confirmation of the modified DNA confirmed the expected $7980 \mathrm{~g} / \mathrm{mol}$ molecular weight of the sequence with the appropriate alkyne modifier (appendix figure D.1).

\subsubsection{Incorporation of the azide modifier into the cocaine split aptamer}

The azide modifier was incorporated into the cocaine split aptamer over a two-step

process, using the same split aptamer as the Heemstra group. ${ }^{149}$ The cocaine split aptamer was first synthesized using a 3'-amino modifier synthesis column with a C7 alkyl linker to the subsequent base in the sequence. After synthesis, under standard conditions and 
purification, a yield of 1143 nmol was attained over six synthesis columns. This was a high synthesis yield, and is likely a result of no phosphoramidite modifiers being included in the synthesis and the short 14 base sequence. Synthesis of this probe was confirmed through ESI-MS molecular weight confirmation, which demonstrated the expected mass of $4241 \mathrm{~g} / \mathrm{mol}$ for the probe with the amine modifier (appendix figure D.2).

A portion of the amino modified sequence was reacted with 3-azidobutryic acid NHS-ester to incorporate the azide modifier. From 500 nmol of the amino modified sequence, 120 nmol of azide modified sequence was recovered after washing and purification. ESI-MS molecular weight confirmation reported the expected molecular weight of $4353 \mathrm{~g} / \mathrm{mol}$, confirming the production of the azide modified cocaine sequence.

\subsubsection{Challenges associated with fluorescent copper nanoparticles and reaction} concentration

Due to the simplicity of the cocaine sensor system, minimal preparatory work was required to delve into sensor evaluation. There is no need to evaluate the background reaction in the absence of cocaine, as under those conditions the split aptamer fragments will not be in proximity and no reaction is expected to occur. Initial experiments focused on minimizing any potential background reaction that could occur between the azide and alkyne modifiers based on concentration effects. The goal of an oligonucleotide templated ligation reaction is to make the background reaction improbable based on minimizing collisions at the concentration that the reaction is run at. Based on previous experiments, background reactions had been observed at concentrations in excess of $10 \mu \mathrm{M}$. Therefore, 
the starting point for testing of the reaction with the labeled probes was a concentration of $1 \mu \mathrm{M}$ for each of the split aptamer fragments in solution. Based on the experiment of Sun et al. where the fluorogenic reaction was developed for applications in DNA ligation chemistry, a concentration of $2 \mathrm{mM} \mathrm{CuSO}_{4}$ and $4 \mathrm{mM}$ sodium ascorbate was required in order to allow the click ligation at this DNA concentration. ${ }^{221}$ The first experiment performed is shown in Figure 5.4, and consisted of testing each of the sequences alone as well as the reaction of azide and coumarin labeled probes in the presence and absence of cocaine. The expected fluorescence spectrum at an excitation wavelength of $333 \mathrm{~nm}$ would give a peak centered at $410 \mathrm{~nm}$ for the triazole-coumarin fluorophore. ${ }^{151,153,155}$ Unfortunately Figure 5.4 shows that this was not the case, and instead a significant peak in the $450 \mathrm{~nm}$ and $470 \mathrm{~nm}$ range was observed along with a small $410 \mathrm{~nm}$ peak. Minimal fluorescence signal change at $410 \mathrm{~nm}$ was observed, indicative of unforeseen issues with the simple reaction setup that had been proposed.

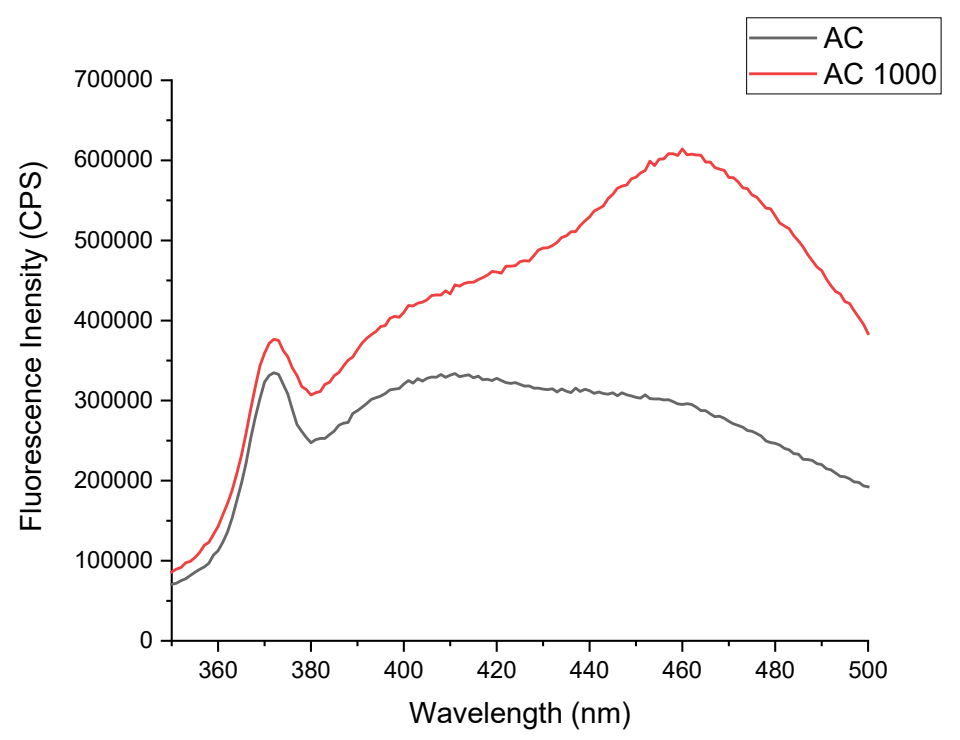


Figure 5.4: Fluorescence spectra of reaction consisting of $1 \mu \mathrm{M}$ of each of azide labeled and coumarin labeled cocaine split aptamer reacted for one hour in the presence (AC 1000) of $1 \mathrm{mM}$ ochratoxin $\mathrm{A}$ and absence of toxin (AC). This initial experiment demonstrated concerns with nanoparticle formation and low reaction concentrations leading to a further investigation of the extraneous peak observed.

To determine the source of the issue, a literature survey of click reactions and issues encountered was undertaken. There was limited mention of any aberrant background fluorescence in literature, but it was discovered early that DNA has a propensity for catalyzing the formation of fluorescent copper nanoparticles in the presence of copper sulfate and sodium ascorbate. Double-stranded DNA has been shown to be metalized by copper(0), acting as a nucleus for the formation of copper nanoparticles. ${ }^{222}$ Single stranded DNA also shows a propensity for the formation of copper nanoparticles, especially in thymine rich sequences. ${ }^{222-223}$ Two common fluorescence emission spectra have been observed for fluorescence copper nanoparticles in literature, being $449 \mathrm{~nm}$ and $575 \mathrm{~nm}$ with an excitation wavelength of $330 \mathrm{~nm}^{223-225}$ The formation of copper nanoparticles catalyzed by DNA could result in the formation of turbid solutions that inhibit light transmission, thereby resulting in decreased signal as observed. This would also result in the formation of a $450 \mathrm{~nm}$ peak that could mask the $410 \mathrm{~nm}$ peak of the expected fluorophore.

The formation of fluorescent copper nanoparticles had still not been conclusively demonstrated in this system, and therefore a transmission electron microscopy experiment was performed. The mixture of azide and coumarin labeled cocaine split aptamer with 4 $\mathrm{mM}$ sodium ascorbate and $2 \mathrm{mM} \mathrm{CuSO}_{4}$ was allowed to react for 1 hour and then deposited onto a copper grid for imaging. As shown in Figure 5.5, copper nanoparticles are observed in the $8-10 \mathrm{~nm}$ range within the sample. The presence of high levels of buffer salts and 
cocaine made acquisition of the TEM difficult, but nanoparticles are clearly visible in the figure shown. In the future, nickel TEM grids should be used to allow for the confirmation of the nanoparticles as copper nanoparticles by EDS, but based on the reaction conditions used it can be assumed that the formed nanoparticles are in fact copper.

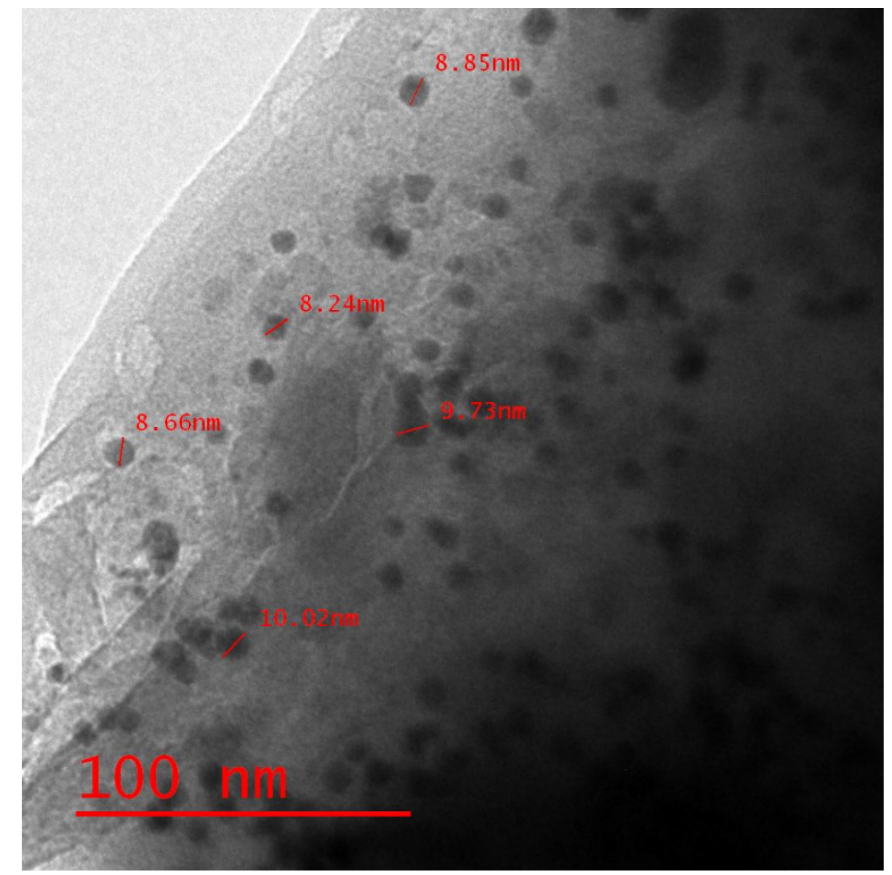

Figure 5.5: Transmission electron microscope image on a copper grid of the reaction of $1 \mu M$ azide and coumarin labeled split aptamer with $1000 \mu \mathrm{M}$ cocaine in the presence of $2 \mathrm{mM} \mathrm{CuSO}_{4}$ and $4 \mathrm{mM}$ sodium ascorbate. Fluorescent copper nanoparticles in the 8-10 $\mathrm{nm}$ range were observed from the reaction, confirming the previous suspicions of their presence.

Methods for the removal of the observed copper nanoparticles were explored to improve the background signal. One strategy is shown in Figure 5.6, where different desalting and filtering strategies were explored for post-reaction. Traditional desalting methods such as ethanol precipitation, desalting Amicon filters, and Glen Pak reverse phase purification cartridges were explored in the presence and absence of cocaine to determine if the $450 \mathrm{~nm}$ peak could be eliminated, and the relative intensity of the $410 \mathrm{~nm}$ 
product peak enhanced. It can be seen from Figure 5.6 that each of these methods appear to be quite lossy, as it would be expected that all sequences in the presence of cocaine have a significantly higher fluorescence intensity than just the azide and coumarin sequences mixed together $(\mathrm{AC})$. None of these strategies were adopted for the final reaction as the loss of ligated DNA could not be controlled and would result in errors in the sensor.

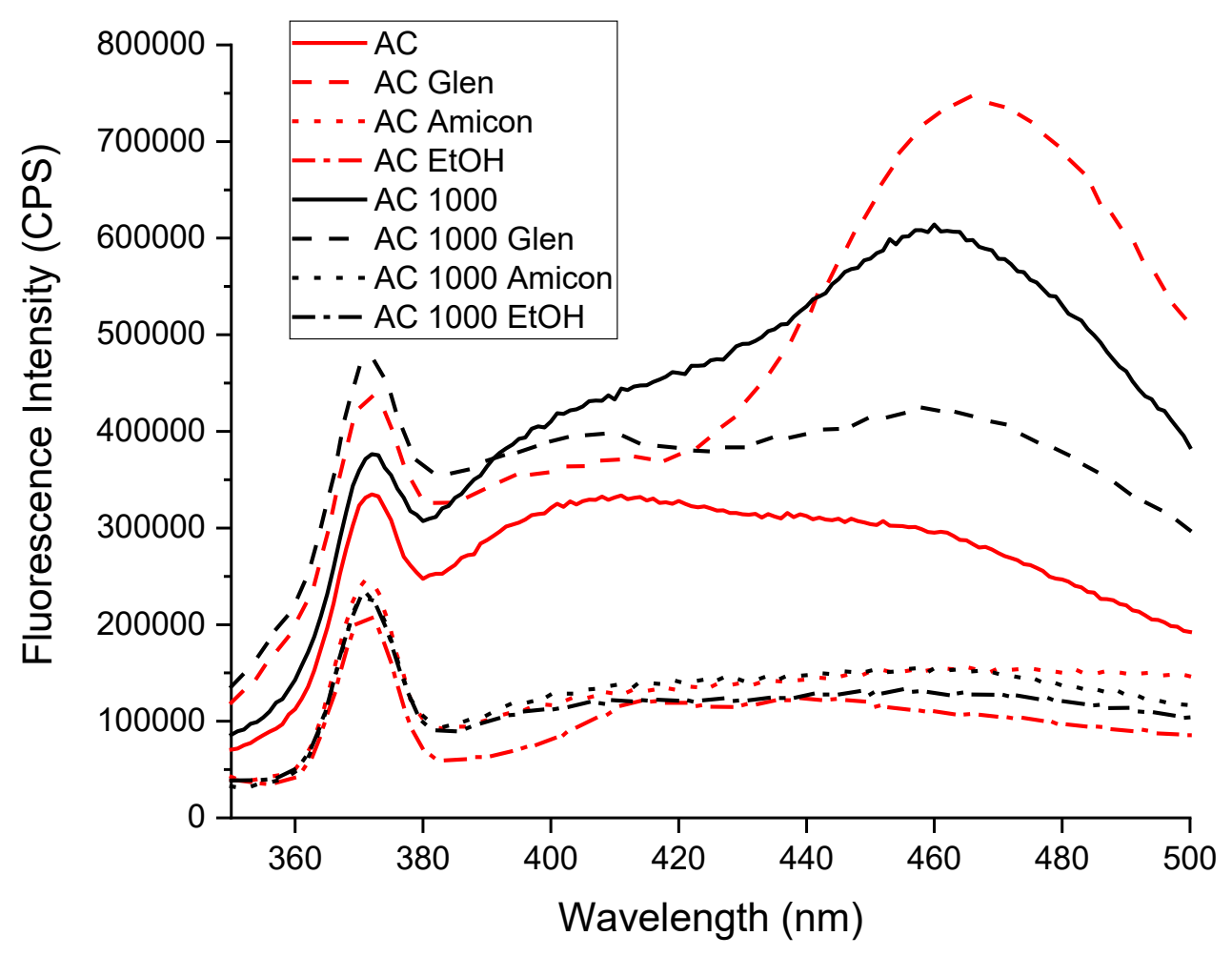

Figure 5.6: Assessment of desalting methods to remove the $450 \mathrm{~nm}$ peak assumed to be associated with fluorescent copper nanoparticles. AC stands for azide and coumarin labeled DNA mixed together, while 1000 is $1000 \mu \mathrm{M}$ cocaine. Desalting after the reaction by Glen Pak cartridge, Amicon filter, and ethanol precipitation were explored. Desalting reactions were abandoned after the results of this test, as the $450 \mathrm{~nm}$ contaminant peak could not be removed under any condition, and the loss of the target $410 \mathrm{~nm}$ peak presented downstream sensor application issues due to lack of reproducibility. 


\subsubsection{Detection of cocaine with the cocaine split aptamer sensor system}

Having identified the formation of fluorescent copper nanoparticles as being the likely cause of the issues encountered in the fluorescence spectra of the reaction, strategies for minimizing its effect were explored. A water-soluble click ligand was added to the reaction in the form of THPTA, as they are designed to help stabilize the requisite $\mathrm{Cu}(\mathrm{I})$ species for the azide-alkyne cycloaddition. ${ }^{142}$ This should help to prevent the formation of copper(0), and thereby limit the ability of copper nanoparticles to form. Additionally, the concentration of copper sulfate for the ligation reaction was explored and it was determined to be much higher than that in most published records of azide-alkyne cycloadditions for the modification of DNA. It is generally advised that a 25 -fold excess of copper sulfate and 40-fold excess of sodium ascorbate relative to the DNA concentration is used in the reaction. It was also decided that the concentration of DNA used for the reaction is likely too low- resulting in the low signal at $410 \mathrm{~nm}$. Therefore, the concentration of the reaction was increased from $1 \mu \mathrm{M}$ of each of the split aptamer fragments, to $10 \mu \mathrm{M}$. Overall, the reaction was amended to split aptamer concentrations of $10 \mu \mathrm{M}$ each with $250 \mu \mathrm{M} \mathrm{CuSO}_{4}$, $250 \mu \mathrm{M}$ THPTA, and $400 \mu \mathrm{M}$ sodium ascorbate. Varying concentrations of cocaine were added to each reaction to determine its applicability as a cocaine sensor, as shown in Figure

\section{7 .}




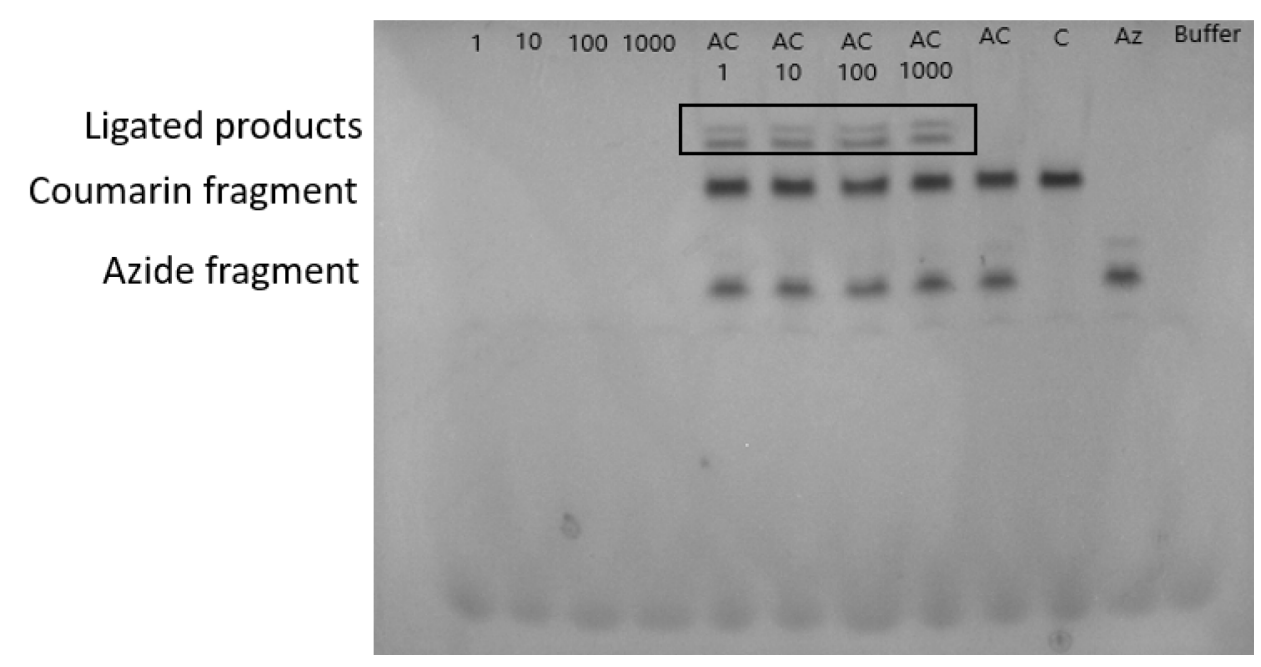

Figure 5.7: Polyacrylamide gel (19\%) of the reaction between the cocaine coumarin fragment (C) and the azide cocaine fragment $(\mathrm{Az})$. Cocaine was added to the reactions at the indicated concentrations in $\mu \mathrm{M}$ (AC 1000- $\mathrm{AC}$ 1). Lanes labeled 1-1000 are for cocaine alone with no added DNA. A ligated product is observable in the reaction lanes containing cocaine and is indicated by a black box.

The results shown in Figure 5.7 are quite promising, as they indicate that there is a ligation reaction occurring to produce a larger band in the $\mathrm{AC} 1000-\mathrm{AC} 1$ lanes (indicative of cocaine concentrations between $1000 \mu \mathrm{M}$ and $1 \mu \mathrm{M}$ ). The ligated product is not observed in the lane containing the base reaction in the absence of cocaine, AC. No bands are observed in the lanes containing cocaine alone (lanes labeled $1000-1$ ), indicating that the newly formed band in the reaction lanes containing cocaine are not a result of the cocaine. As a preliminary step towards the development of a sensor, this showed promise, and was followed up with a more extensive analysis of cocaine concentrations shown in Figure 5.8, Figure 5.9, and Figure 5.10. 


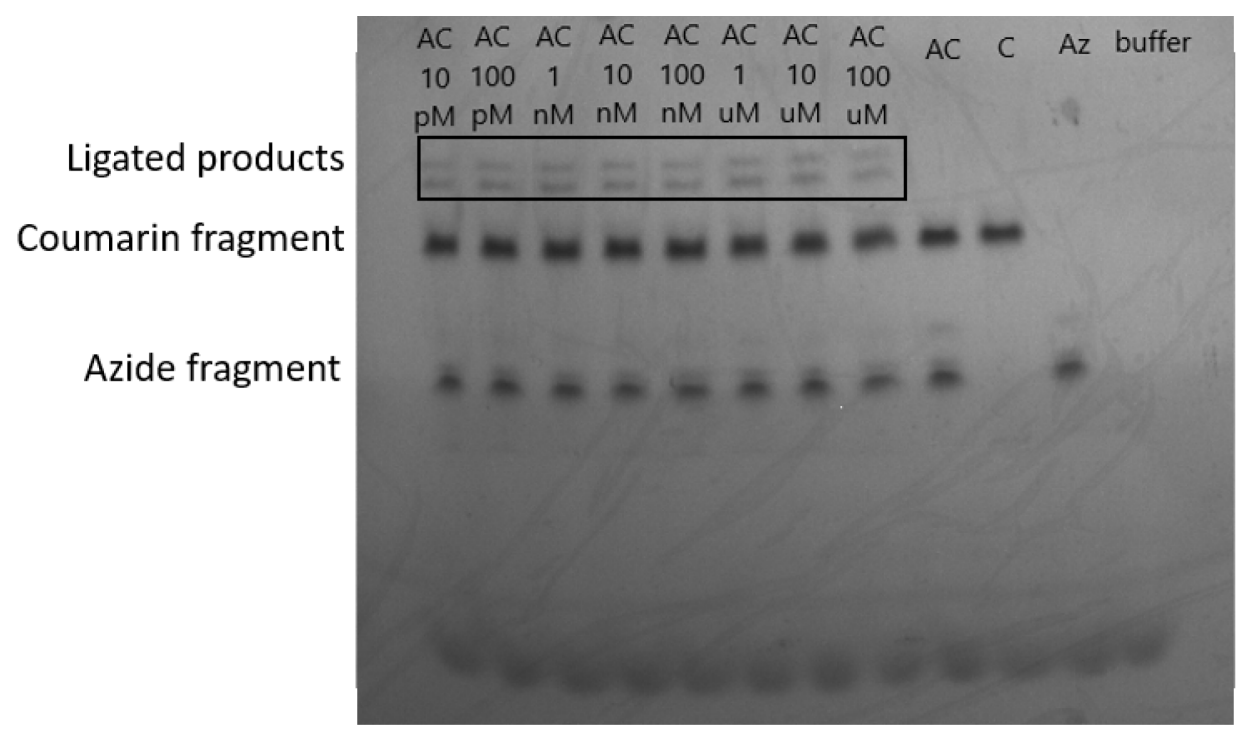

Figure 5.8: Polyacrylamide gel (19\%) for the reactions in the presence of cocaine (AC $100 \mu \mathrm{MM}-\mathrm{AC}$ $10 \mathrm{pM}$ ), or absence of cocaine (AC). Lanes $\mathrm{C}$ and $\mathrm{Az}$ represent the coumarin cocaine fragment, and the azide cocaine fragment respectively. A ligated product band is observable in all lanes containing cocaine with the split aptamer (black box), but not in the lanes missing any of the components.

Again, all reaction lanes run in the presence of cocaine (AC $100 \mu \mathrm{M}-\mathrm{AC} 10 \mathrm{pM})$ showed the formation of a new band that was heavier than either of the azide or coumarin bands. No new bands were observed in the lanes not containing cocaine, indicating that the newly formed band is a result of the presence of cocaine. Interestingly, there does not appear to be significant variation in the intensity of the observed new band. It would be expected that more product would be formed in the lanes containing higher levels of cocaine, and the band intensity should decrease from right to left across the gel. ESI-MS molecular weight confirmation demonstrated the formation of the target ligated aptamer sequence with a molecular weight of $12030.9 \mathrm{~g} / \mathrm{mol}$ (appendix figure D.4) when both product bands were sent for analysis. Additional analysis is required to completely characterize the other products observed by ESI-MS analysis of the ligated product. It was 
assumed that the two bands present might be a result of cocaine bound and cocaine unbound product not separating by gel electrophoresis, but further analysis is required since this was refuted by ESI-MS. Glaser coupling of the terminal alkyne coumarin was also explored as a possibility, but the observed high molecular weight products do not correspond to this outcome either. Decomposition pathways of the alkyne-coumarin or the triazole ligated product should also be explored in further detail in order to deduce the identity of the ESI-MS low molecular weight peaks as well.

Fluorescence spectroscopy of the reactions shown in Figure 5.8 are depicted in Figure 5.9. In this case there is no interfering $450 \mathrm{~nm}$ peak, likely as a result of decreased copper nanoparticle formation and increased fluorophore formation from the higher DNA concentration. Limited data can be pulled from this version of the plot, but it can be observed that the fluorescence of all reactions in the presence of cocaine are significantly higher than the fluorescence of the reactions in the absence of cocaine (AC, coumarin, azide). 


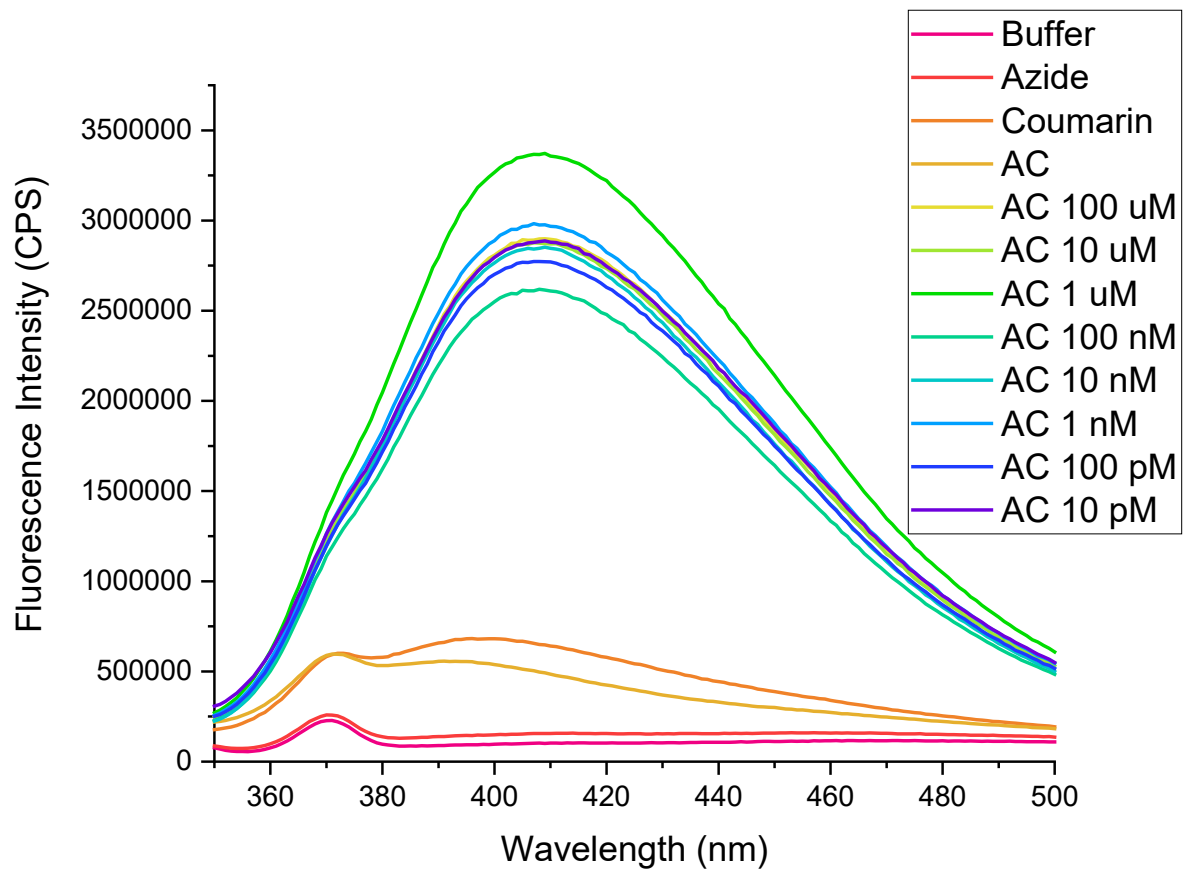

Figure 5.9: Fluoresence emission spectra measured between $350-500 \mathrm{~nm}$ with an excitation of $333 \mathrm{~nm}$. AC represents the azide and coumarin cocaine fragments mixed together, while $\mathrm{AC} 100 \mu \mathrm{M}$ to $\mathrm{AC} 10$ pM represent the reaction in the presence of cocaine at the indicated concentration. The reaction in the absence of cocaine (AC) resulted in a similar low signal to the coumarin alone $(C)$, while the addition of cocaine saw a positive increase in fluorescence intensity with cocaine concentration in the reaction system.

A closer look at the trend in fluorescence at $410 \mathrm{~nm}$ in the presence and absence of cocaine is shown in the bar graph in Figure 5.10. The fluorescence intensity of the reaction in the absence of cocaine is significantly lower than the fluorescence intensity of all the reactions containing cocaine across the range of $100 \mu \mathrm{M}$ to $10 \mathrm{pM}$. No trend is observable for the fluorescence intensity in the presence of cocaine along the series. It would therefore have to be concluded that this system is a turn-on qualitative sensor that indicates the presence of cocaine but cannot be used for the quantification of cocaine due to the lack of trend. 


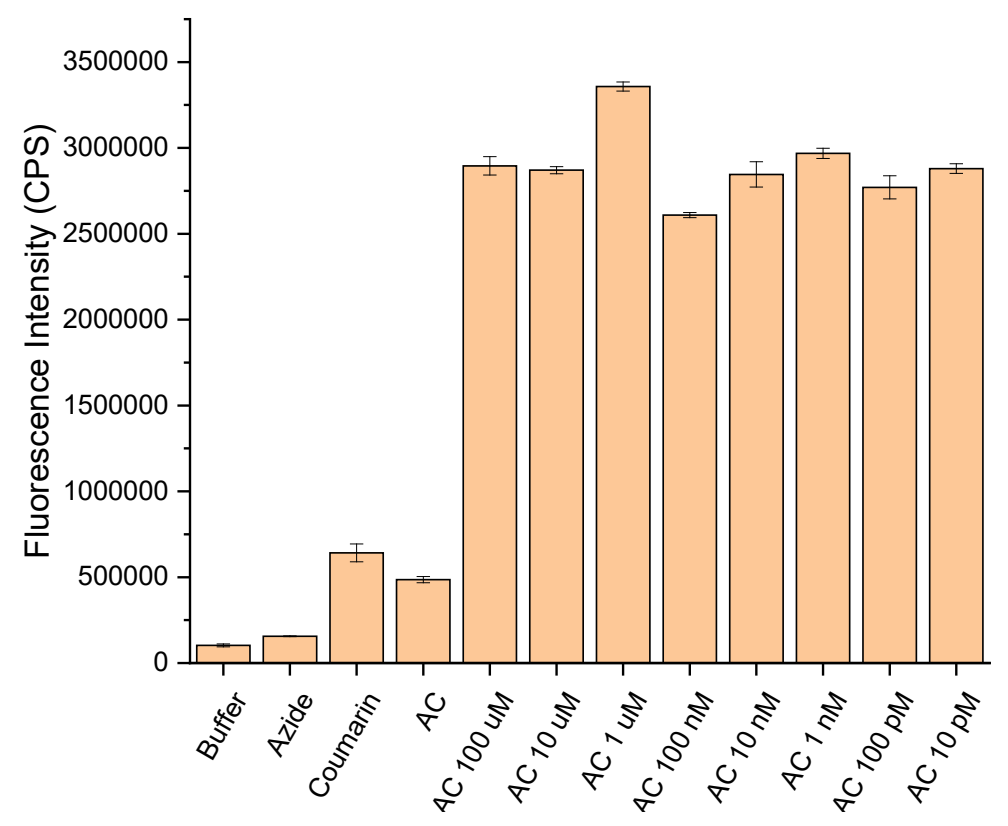

Figure 5.10: Bar plot conversion of the data from Figure 5.9 showing the measured fluorescence intensity at $410 \mathrm{~nm}$ for each of the reactions. The reaction in the absence of cocaine (AC) resulted in a similar low signal to the coumarin alone (C), while the addition of cocaine saw a positive increase in fluorescence intensity with cocaine concentration in the reaction system. Data was collected in triplicate $(\mathbf{N}=3)$.

It was unexpected that this system would result in a qualitative sensor with such a broad range of detection of cocaine. In all reactions run, the combination of the azide split aptamer and the coumarin split aptamer in the absence of cocaine results in minimal fluorescence from the fluorogenic reaction as well as the absence of the ligated band by gel electrophoresis. This system is specific to cocaine, in that in the absence of cocaine no ligation event occurs and therefore results in a minimal background fluorescence. In the presence of cocaine, no observable trend can be seen for the fluorescence as had been seen in previous systems for QUAL detection of ochratoxin A and fluorogenic click reaction for the detection of thrombin in the tripartite or single aptamer system. Analysis of the gel in 
Figure 5.8 also shows that the reaction seems to stop before complete consumption of the azide or coumarin sequences, as would have been expected if the system had reached a plateau with their full consumption resulting in constant fluorescence output. It therefore must be assumed that there is some form of competing interaction that is resulting in a plateau in the fluorescence output upon addition of cocaine to the system. It is possible that the addition of cocaine could change the geometry of the reactive groups sufficiently through stabilization of the split aptamer duplex and with such high turn-over from the rapid reaction that even small amounts of cocaine result in significant fluorescence product. If this were the case, it would be expected that what we are observing is the plateau and that the linear detection range is below the $10 \mathrm{pM}$ concentration tested. This does not explain the reaction appearing to stop by gel and warrants further exploration in downstream studies.

When the designed cocaine sensor is compared to literature, the qualitative detection region alone is wider than that demonstrated for most cocaine aptasensors and the limit of detection is still unknown due to the plateau. An early example from literature showed a colourimetric gold-nanoparticle assays for cocaine which demonstrated a dynamic range of $20 \mathrm{nM}$ to $200 \mathrm{mM}$ with a limit of detection of $2 \mu \mathrm{M}$. ${ }^{208}$ This explores cocaine concentrations well above the range that was tested in this experiment, but it can be assumed that based on the range tested the limit of detection shown here should be much lower once the linear range is established. Fluorescence based cocaine aptasensors have been established, with one example resulting in a dynamic range of 0.2-100 $\mathrm{mM}$, with a limit of detection of $190 \mathrm{nM} .^{226}$ Electrochemical methods for cocaine detection have been 
established with low dynamic ranges and limits of detection of $14.5 \mathrm{fM}-14.5 \mathrm{pM}$ and 7.8 fM respectively. ${ }^{227}$ This system could challenge an electrochemical system once the observed issues are explored further and serves to demonstrate the potential of fluorogenic split aptamers for sensitive analyte detection. Further studying the reaction times to determine if the sensor is more useful at times of less than 30 minutes could open the applications of the sensor to other fields where rapid readouts are essential.

\subsubsection{Controls and specificity of the cocaine split aptamer sensor system}

To assess the validity of the designed cocaine sensor, a series of control reactions were run. In one form of control reaction, cocaine was substituted with either thrombin, ATP, or adenosine to determine if the fluorescent product forms selectively in the presence of cocaine or if any small molecule would result in a fluorescence increase. In another test, the azide modified thrombin probe SRA_2 from chapter 4 was added to the reaction in place of the azide-cocaine modifier in the presence and absence of cocaine to see if there is sequence selectivity in the aptamer, or if the reaction is solely based on the fluorogenic ligation regardless of target presence or DNA sequence. The fluorescence spectra are shown in Figure 5.11, where the highest fluorescence output is in the reaction between the azide and cocaine labeled split aptamers and $10 \mu \mathrm{M}$ cocaine. All other control reactions including the mismatched azide resulted in a similar low-level fluorescence. 


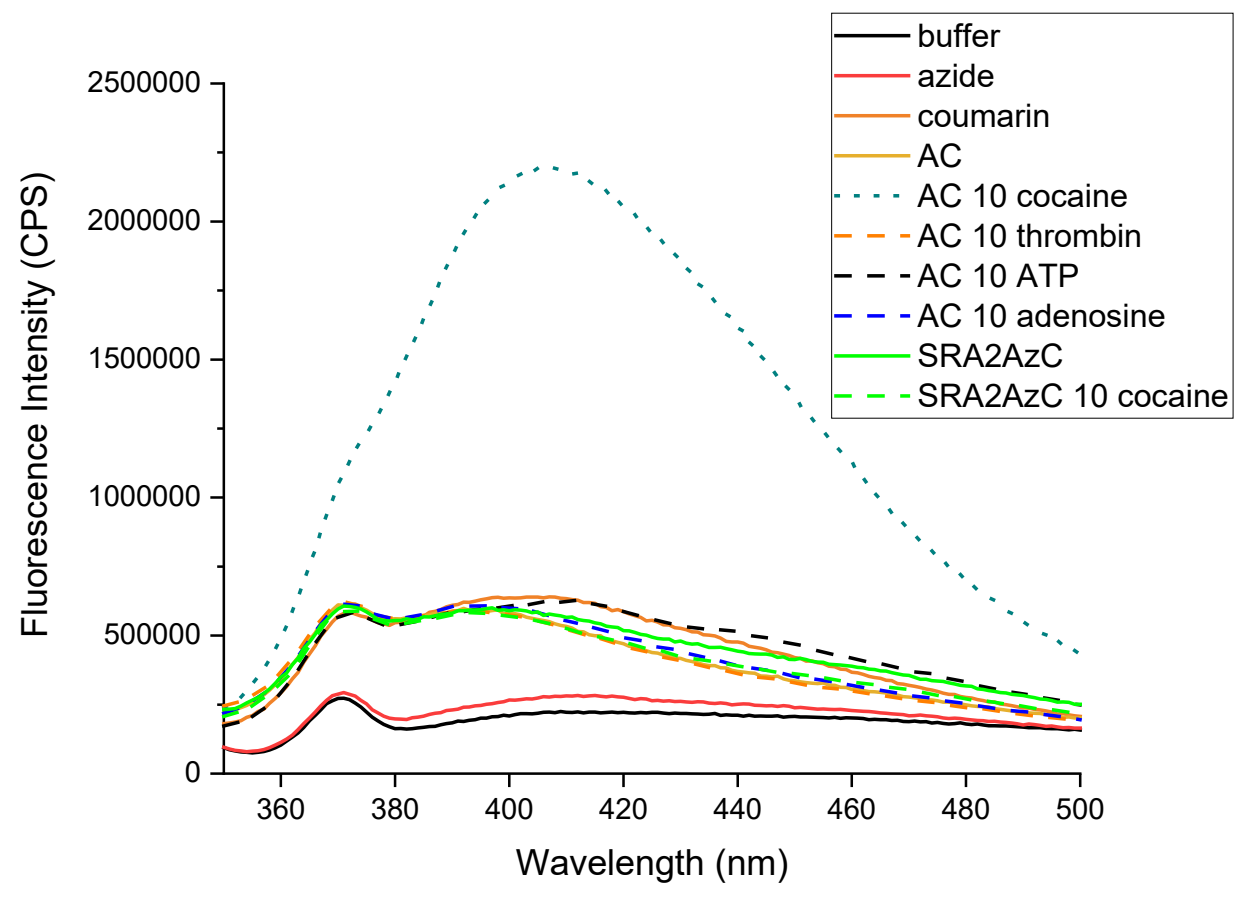

Figure 5.11: Fluoresence emission spectra measured between 350-500nm with an excitation of $333 \mathbf{~ n m}$. AC represents the azide and coumarin cocaine fragments mixed together, while AC 10 cocaine, thrombin, ATP, and adenosine represent the reaction in the presence of $10 \mu \mathrm{M}$ of the indicated target. SRA_2Az represents reactions where the thrombin azide probe is used in place of the cocaine azide fragment. The reaction in the presence of $10 \mu \mathrm{M}$ cocaine resulted in a large fluorescent signal, while the control reactions all resulted in a low signal comparable to coumarin alone. Replacement of the azide sequence with the thrombin azide resulted in a low signal as well, indicative of the importance of the complementary cocaine azide split aptamer sequence.

The trend is most easily observed when the data is converted into a bar graph as shown in Figure 5.12. As previously indicated, the fluorescence of the azide-coumarin cocaine reaction in the presence of cocaine significantly dwarfs the signal output from the reaction in the presence of control targets or sequences. This indicates that the designed fluorogenic ligation for the detection of cocaine is highly dependent on the sequence of the DNA used- meaning that it must be the cocaine split aptamer for ligation to occur and is also specific to the cocaine target. 


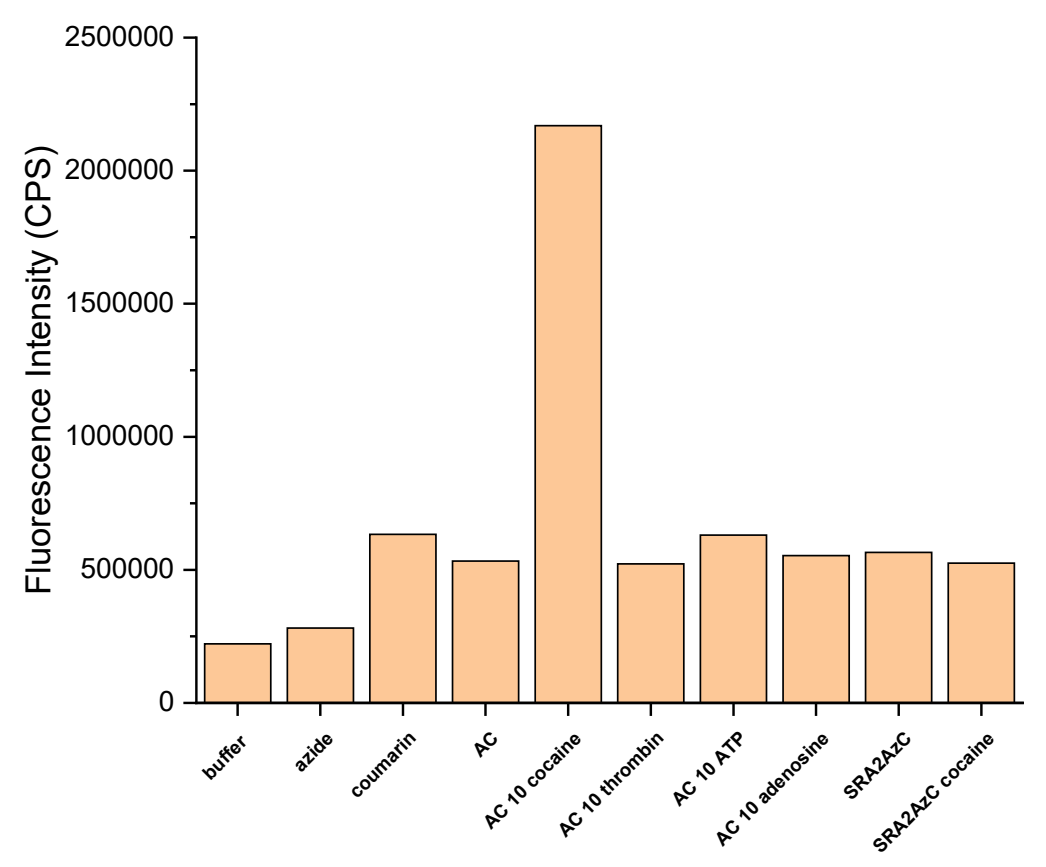

Figure 5.12: Bar plot conversion of the data from Figure 5.11 showing the measured fluorescence intensity at $410 \mathrm{~nm}$ for each of the reactions. The reaction in the presence of $10 \mu \mathrm{M}$ cocaine resulted in a large fluorescent signal, while the control reactions all resulted in a low signal comparable to coumarin alone. Replacement of the azide sequence with the thrombin azide resulted in a low signal as well, indicative of the importance of the complementary cocaine azide split aptamer sequence. Data was collected from a single replicate.

Many studies have demonstrated that the cocaine aptamer is a highly specific system, with the only other molecule showing a high binding affinity to the target being quinine. $^{207,228}$ Cocaine derivatives have been previously established to not show any binding affinity, ${ }^{149}$ and should be explored further as supplemental controls in downstream applications in order to ensure that the cocaine molecule is not undergoing an extraneous 
reaction with the aptamer and aptamer modifiers resulting in the high fluorescence in its presence.

\subsection{Conclusions}

This project brought about two important findings. The formation of copper nanoparticles in azide-alkyne cycloaddition reactions catalyzed by DNA is an important consideration when designing fluorogenic reactions and in other downstream applications that could be envisioned for copper-catalyzed DNA ligation reactions. Future directions should include the exploration of copper freeze azide-alkyne cycloadditions if a simple DNA compatible fluorogenic reaction can be designed and implemented. In this case the impact of the formation of fluorescent copper nanoparticles could be mitigated through a DNA concentration increase along with the incorporation of a water soluble click ligand and a more reasonable ratio of copper:DNA. This was applied to the thrombin fluorogenic system in order to generate the data shown in chapter 4, and warrants further exploration in all copper catalyzed fluorogenic reaction systems.

This also represents the first application of fluorogenic strand ligation to DNA split aptamers, and it has been shown that this encompasses a new avenue for exploring ligation reactions using DNA aptamers. Downstream applications could involve new chemistry that is either chromogenic or fluorogenic or could be used for applications in targeted delivery. In this portion of the project it was demonstrated that cocaine could be detected using a fluorogenic click ligation reaction between the fragments of the cocaine split aptamer. Cocaine could be detected qualitatively between $100 \mu \mathrm{M}$ and $10 \mathrm{pM}$. Future 
applications may explore alternative split aptamers or alternative chemistry to improve the system. An exploration of the reason for the reaction plateau by both gel electrophoresis and fluorescence spectroscopy will also be undertaken. 


\section{Chapter: Significance and future work}

Oligonucleotide templated reactions have existed for many years but there are very few examples of their direct application to the field of DNA aptamers. When this project was initially outlined, the goal was to demonstrate that the two fields do not need to be mutually exclusive, and that a DNA aptamer could be used to control a chemical reaction occurring on the DNA. By controlling a chemical reaction using the target of a DNA aptamer, either through its initiation or its inhibition, this could be turned into either a sensor, or downstream applications in molecular payload delivery with the release of a product such as a drug could be explored. To start, the goal was to demonstrate that controllable oligonucleotide reactions could be used to develop sensors using DNA aptamers as a proof of concept before continuing into payload release applications.

The first application explored was the control of quenched autoligation reactions (QUAL) as had been previously demonstrated by the Kool research group. By designing a system wherein a dabsyl electrophile bearing quenched probe could be hybridized to the complementary ochratoxin A aptamer adjacent to a thiophosphate nucleophile containing probe, a reaction could be initiated in the traditional QUAL ligation reaction style. The purpose of incorporating the ochratoxin A aptamer into the system is to take advantage of target binding to limit the hybridization of the complementary reaction probes and thereby inhibit the ligation reaction. This would result in a decrease in fluorescence intensity relative to the baseline reaction at a given time point, permitting the development of a sensor. A sensor for ochratoxin A was developed using this system with a linear dynamic range of $100 \mu \mathrm{M}$ to $100 \mathrm{pM}$ with a limit of detection of $1.5 \mathrm{pM}$. This places this sensor 
within the regulatory range of ochratoxin A in the European Union and compares quite favourably to other reported ochratoxin A aptasensors. The quenching effect of ochratoxin A on the fluorescence emission in the system remains to be explored, as does assessment of the system in a complex matrix to determine validity for real-world sample analysis. A further exploration of the stability of the dabsyl probe over long term and optimization of its stability should also be undertaken including potential chemical optimization to increase stability within affecting the reaction. This QUAL coupled aptamer sensor system (QUALAPT) could be applied to almost any aptamer to develop a signal on sensor and presents a novel avenue to explore in aptasensor development based on the results seen herein.

After experiencing unexpected challenges due to the lability of the dabsyl probe in the QUAL system, alternative chemistry was explored surrounding fluorogenic click ligations of azide and alkyne-coumarin modified DNA. These modifiers were synthesized and optimized according to a literature protocol, and overall process yields were improved compared to literature. These reactive elements were then used to design three sensor systems: a tripartite thrombin sensor like the QUAL reaction; a single dual modified thrombin aptamer sensor system; and a cocaine split aptamer fluorogenic ligation sensor system. All of the fluorogenic copper-catalyzed reactions performed suffered from issues with the formation of fluorescent copper nanoparticles, which needs to be further evaluated to ensure it is not causing unforeseen complications. Novel chemical modifiers incorporating copper free click chemistry should be rationally designed and synthesized 
for a fluorogenic system to eliminate these side reactions and promote the ability to use these types of reaction in vivo for sensing applications.

The thrombin tripartite reaction sensor system was synthesized according to the same principles as the QUAL sensor system, but using a known structure switching aptamer. A linear dynamic range between $100 \mathrm{nM}$ and $10 \mathrm{pM}$ was observed with a calculated limit of detection of $3.9 \mathrm{fM}$ which compares favourably with existing thrombin sensors. Concerns from the background quenching and further evaluation of controls still need to be explored. The thrombin single aptamer system was designed as the first chemical reaction directly initiated through aptamer-target binding and presents a novel field in aptasensor development and aptamer technology. Incorporation of the azide and alkyne chemical modifiers within the sequence at rationally designed locations based on the known G-quadruplex forming domain of the SRA_2 aptamer permitted the design of a functional sensor. Preliminary results demonstrated the fluorescence signal increase through the addition of thrombin to the single modified aptamer, but challenges with synthetic yield limited an extensive study and requires further examination. The single modified aptamer could be used for many applications where the signal on or molecule release reaction needs to be triggered by the presence of the aptamer target and a multitude of downstream systems could be explored for such chemistry. It could also be envisioned that such a system could be used to elucidate aptamer-target structure when such data is 
unknown. Improved yield in incorporation of reactive modifiers would need to be established first, but this is a relevant side consideration for this system.

The final system explored was the application of fluorogenic click chemistry to split aptamers for initiating a signal-on type reaction. In the presence of the split-aptamer target, the two labeled halves of the aptamer would be brought into proximity and a reaction initiated. It was demonstrated that such a system could be designed using the cocaine split aptamer to develop a qualitative sensor between $100 \mu \mathrm{M}$ and $10 \mathrm{pM}$ cocaine concentrations. A further exploration as to the reason that this system is qualitative instead of quantitative will still need to be performed, as will the assessment of more cocaine relevant control reactions. The success of this system for initiating a reaction that was not occurring in the absence of cocaine demonstrates the utility of end labeling two split aptamer sequences for a signal-on sensor design. This could be applied to any split aptamer in existence and could also be used for the development of new split aptamers and their assessment from known split aptamers.

The ability to control a chemical reaction using a DNA aptamer has been demonstrated in each of these systems. In the tripartite examples, the reaction is inhibited by the presence of the aptamer target, while in the single aptamer system and the split aptamer system target presence initiates the reaction. Simple chemical modifiers with established chemistry from literature were used for this series of proof of concept reactions. The next step will be the rational design of new chemical reactions, particularly those that are click chromogenic reactions that occur in the absence of copper. This would require both a synthetic organic component to the project in the development of novel chemical 
modifiers for DNA that are both compatible with the DNA synthesizer and undergo the desired reaction in biorthogonal conditions. This therefore represents the first step in the creation of a new field of aptasensor and aptamer technology that should be further explored. 


\section{Appendices}

\section{Appendix A : Mass spectra for the Quenched Autoligation sensors}

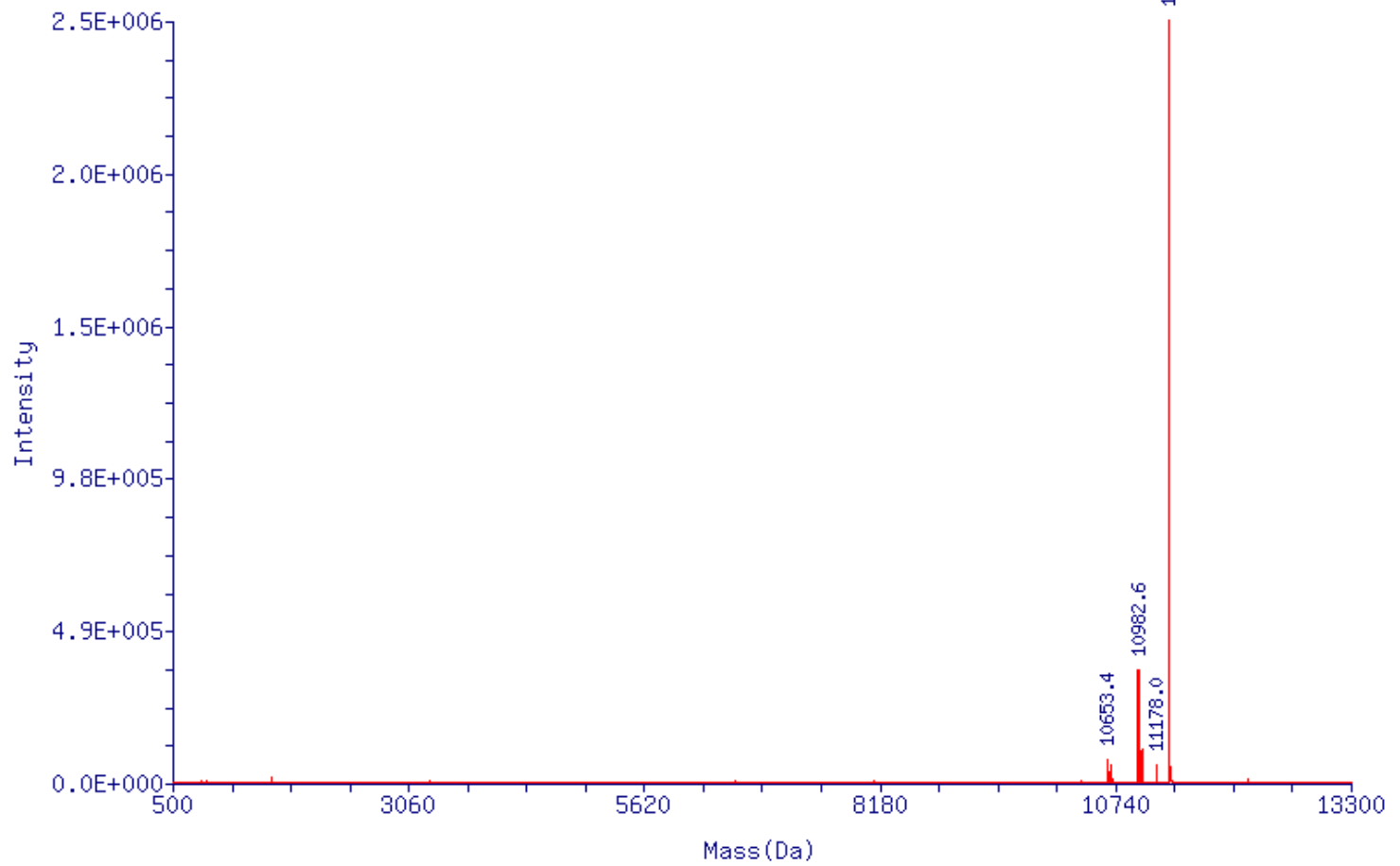

A.1 Deconvoluted mass spectrum of the ochratoxin A aptamer 1.12.2, confirming its expected molecular weight of $11311.9 \mathrm{~g} / \mathrm{mol}$. 


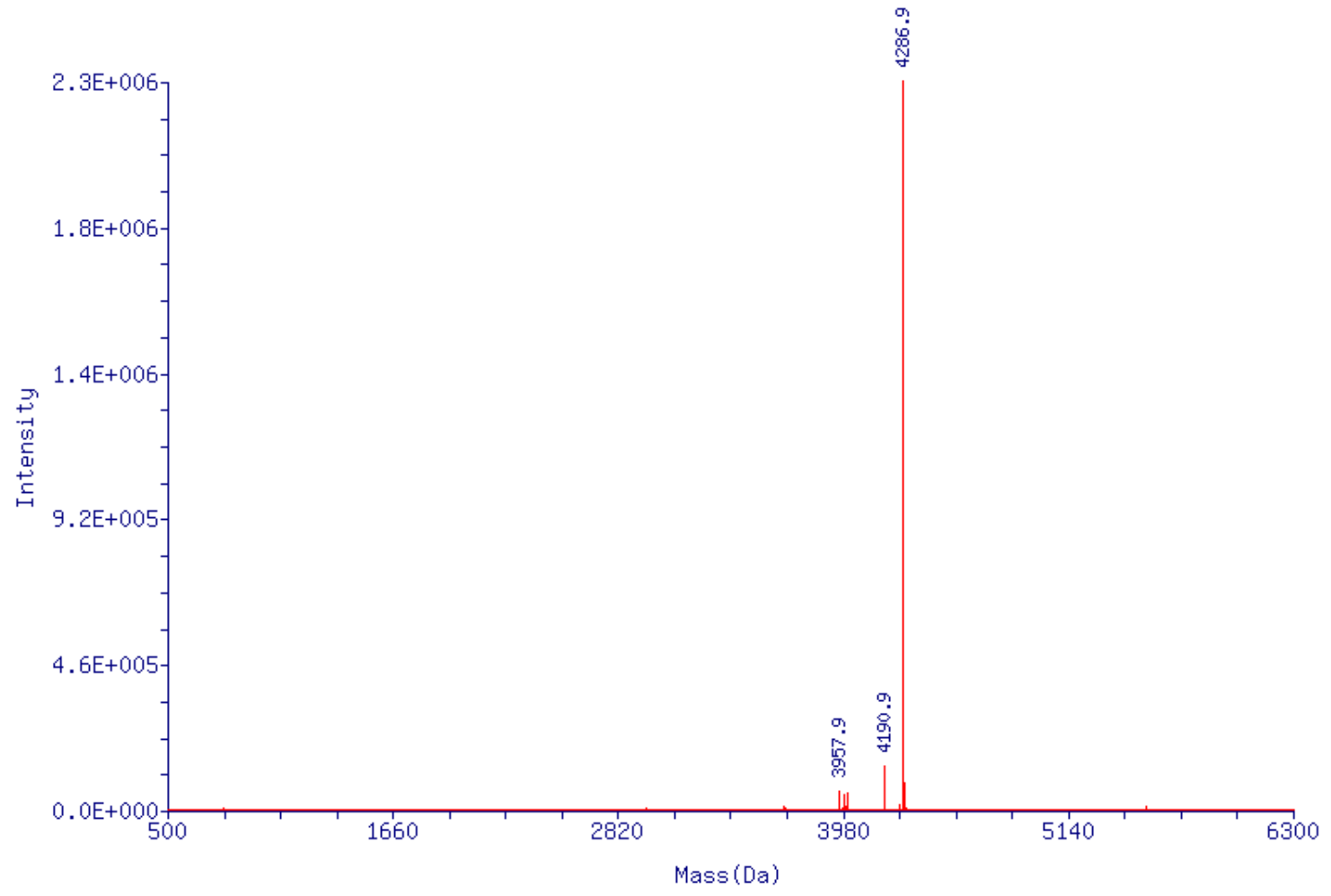

A.2 Deconvoluted mass spectrum of the thiophosphate probe, confirming its expected molecular weight of $4286.9 \mathrm{~g} / \mathrm{mol}$. 


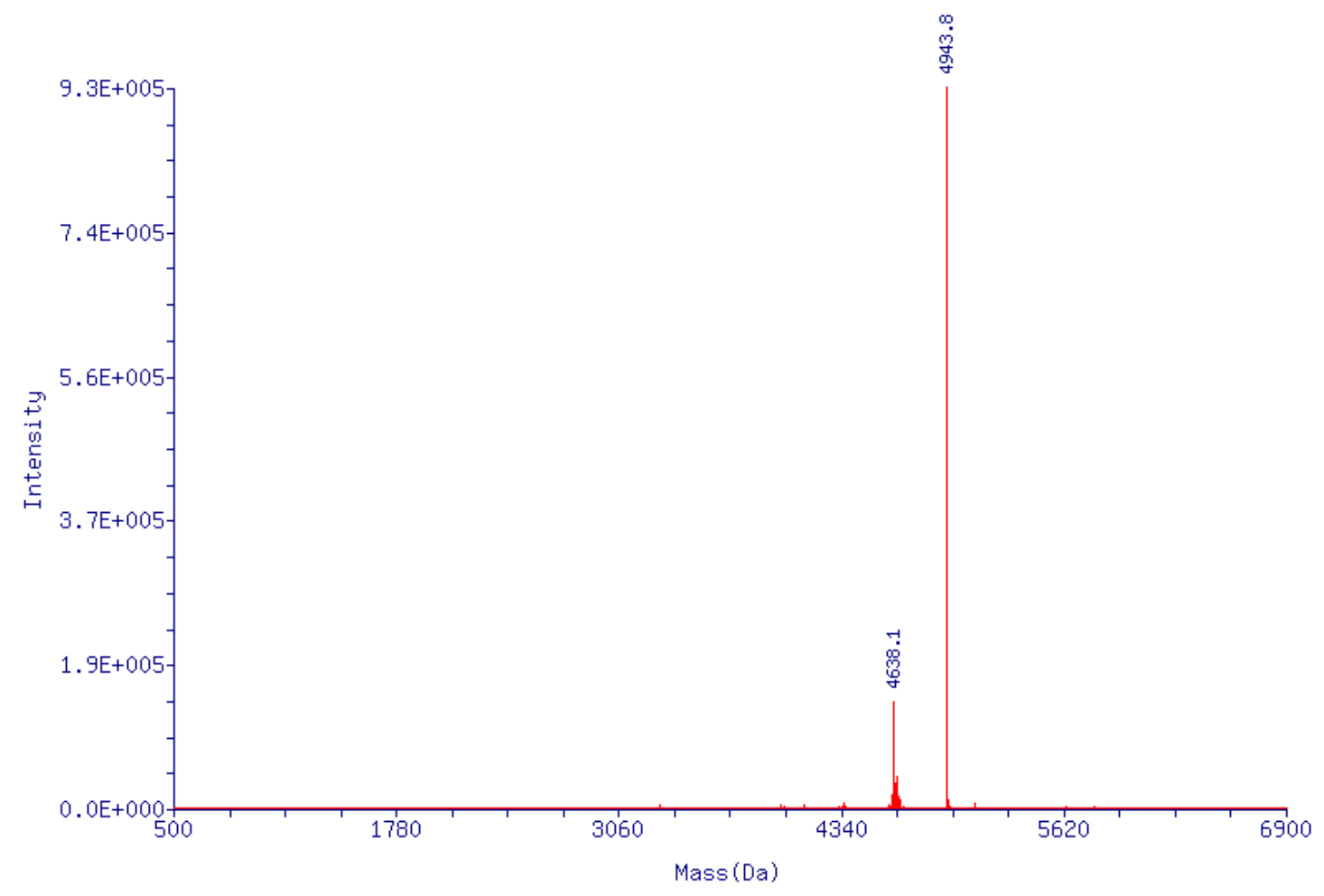

A.3 Deconvoluted mass spectrum of the dabsyl probe, confirming its expected molecular weight of $4943.8 \mathrm{~g} / \mathrm{mol}$.

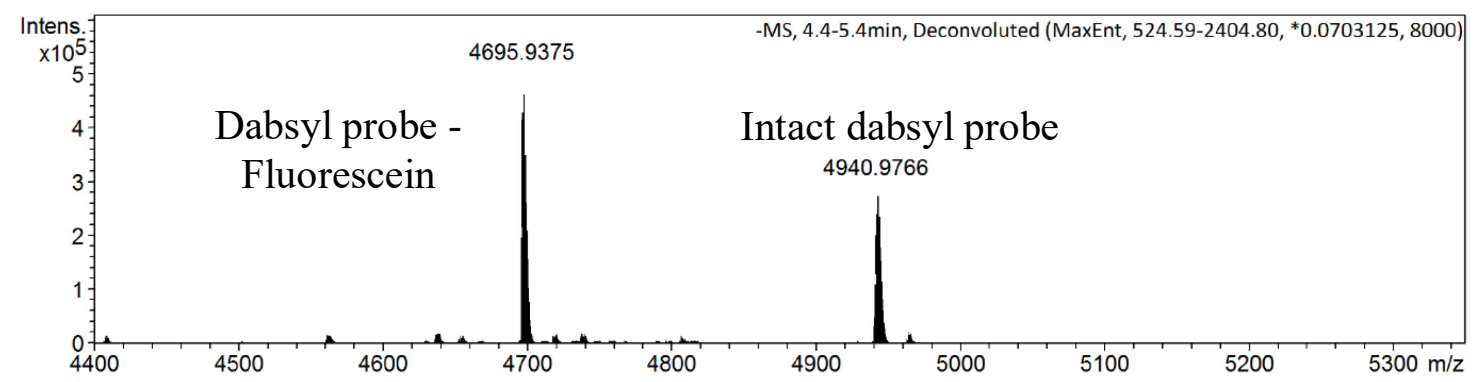

A.4 Deconvoluted mass spectrum of the decomposed dabsyl probe, demonstrating the loss of the dabsyl modifier to give a molecular weight of $4695.9 \mathrm{~g} / \mathrm{mol}$. 


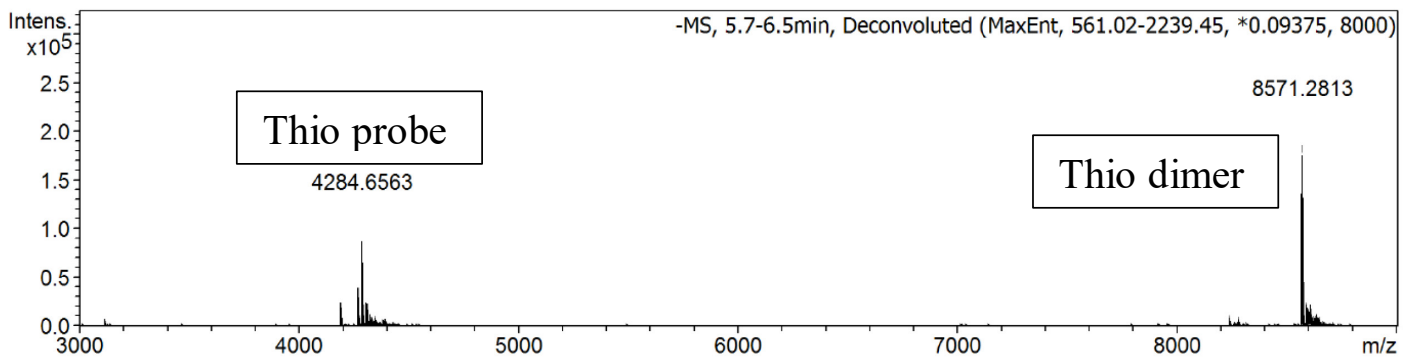

A.5 Deconvoluted mass spectrum of the dimerized thiophosphate probe.

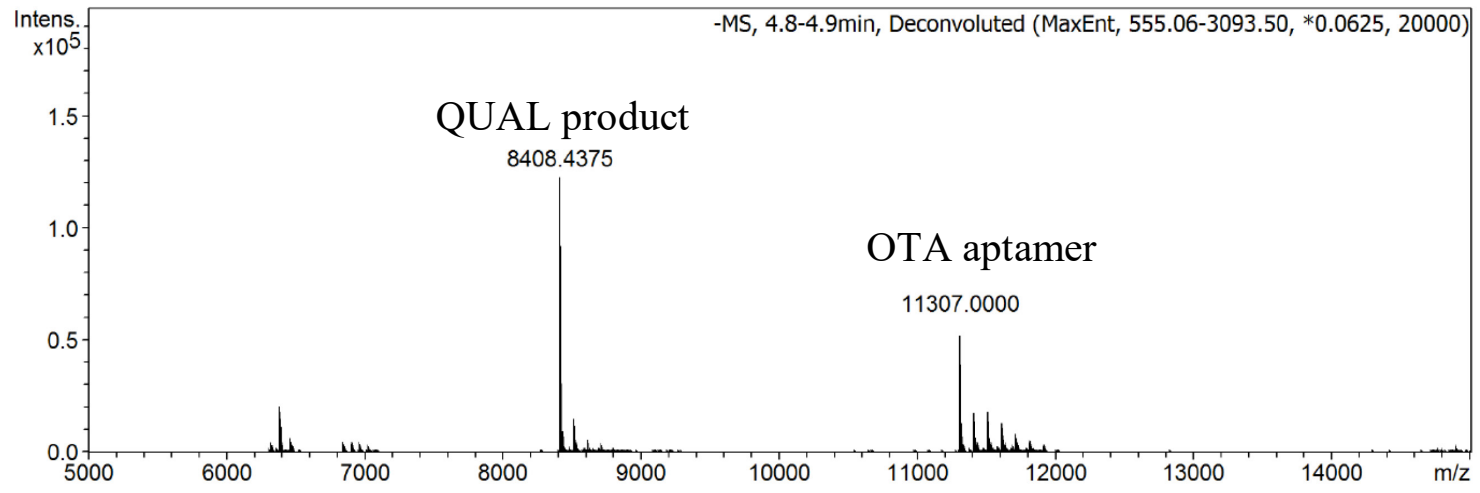

A.6 Deconvoluted mass spectrum of the ligation product excised from a polyacrylamide gel, showing the ligated product at $8408.4 \mathrm{~g} / \mathrm{mol}$, along with the OTA aptamer 


\section{Appendix B NMR spectra}

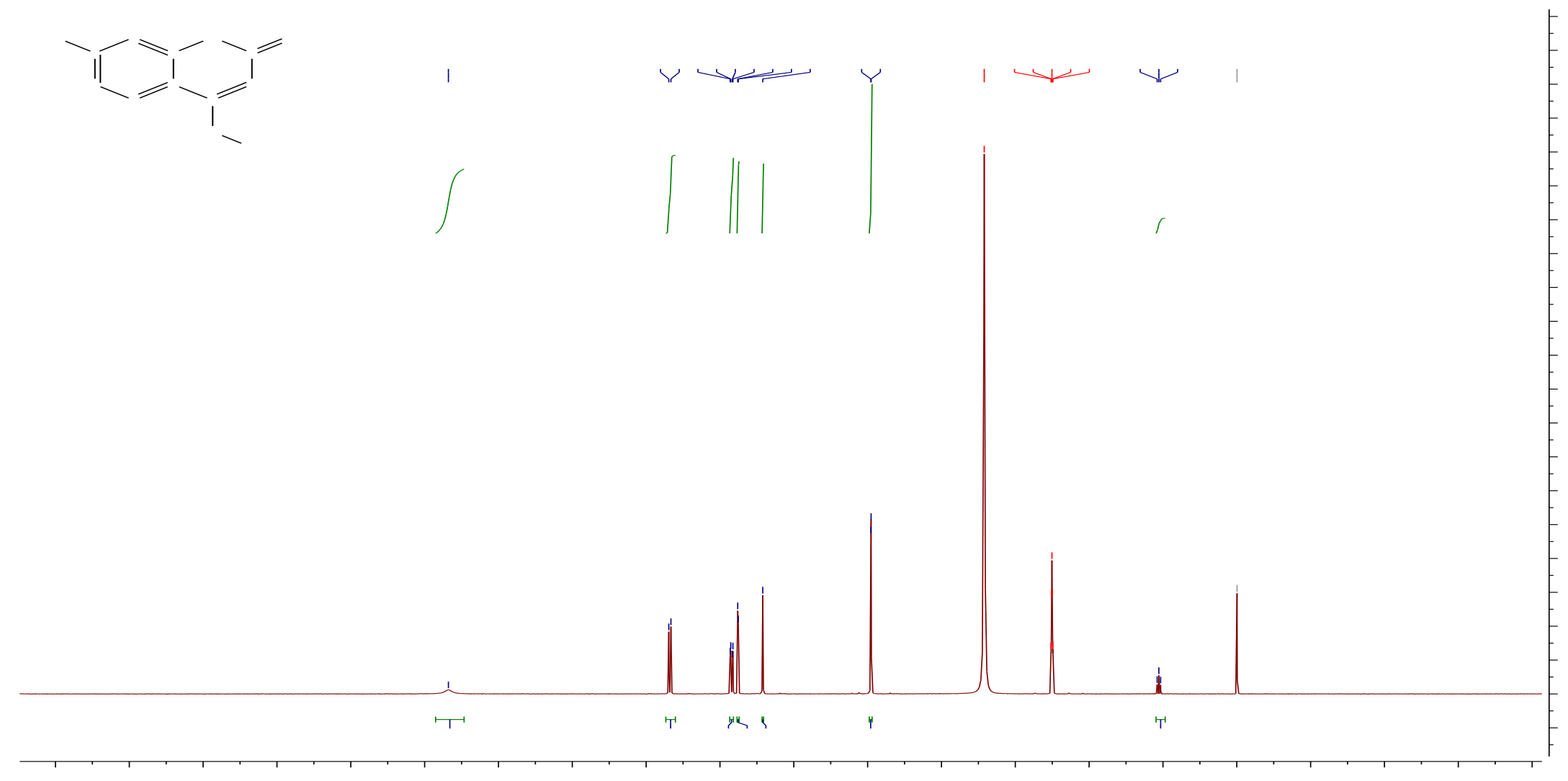

B.1 ${ }^{1} \mathrm{H}$-NMR spectrum of 4-(chloromethyl)-7-hydroxy-2H-chromen-2-one 3.2 in d6-DMSO. 


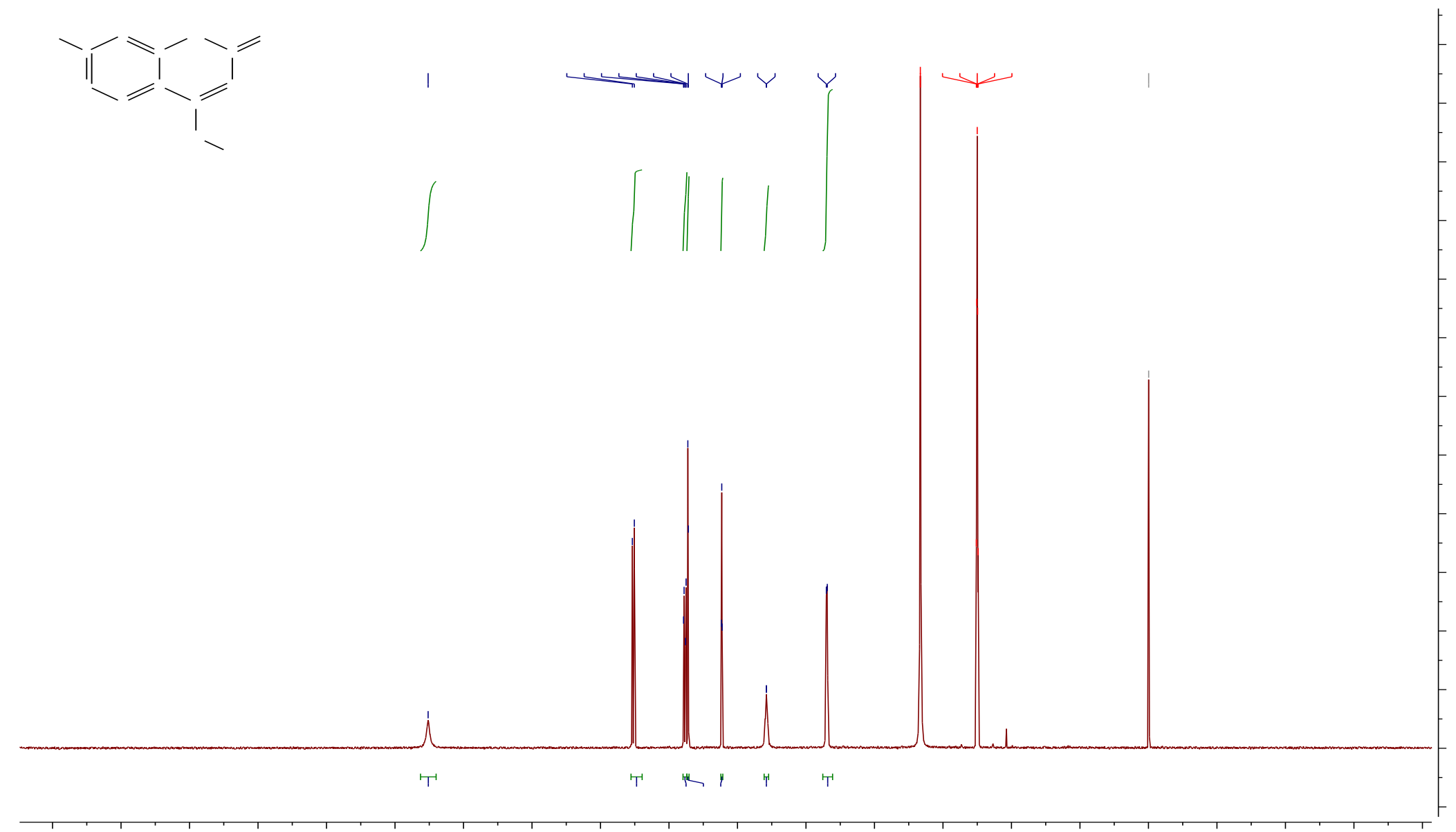

B.2

${ }^{1}$ H-NMR spectrum of 7-hydroxy-4-(hydroxymethyl)-2H-chromen-2-one 3.3 in d6-DMSO. 


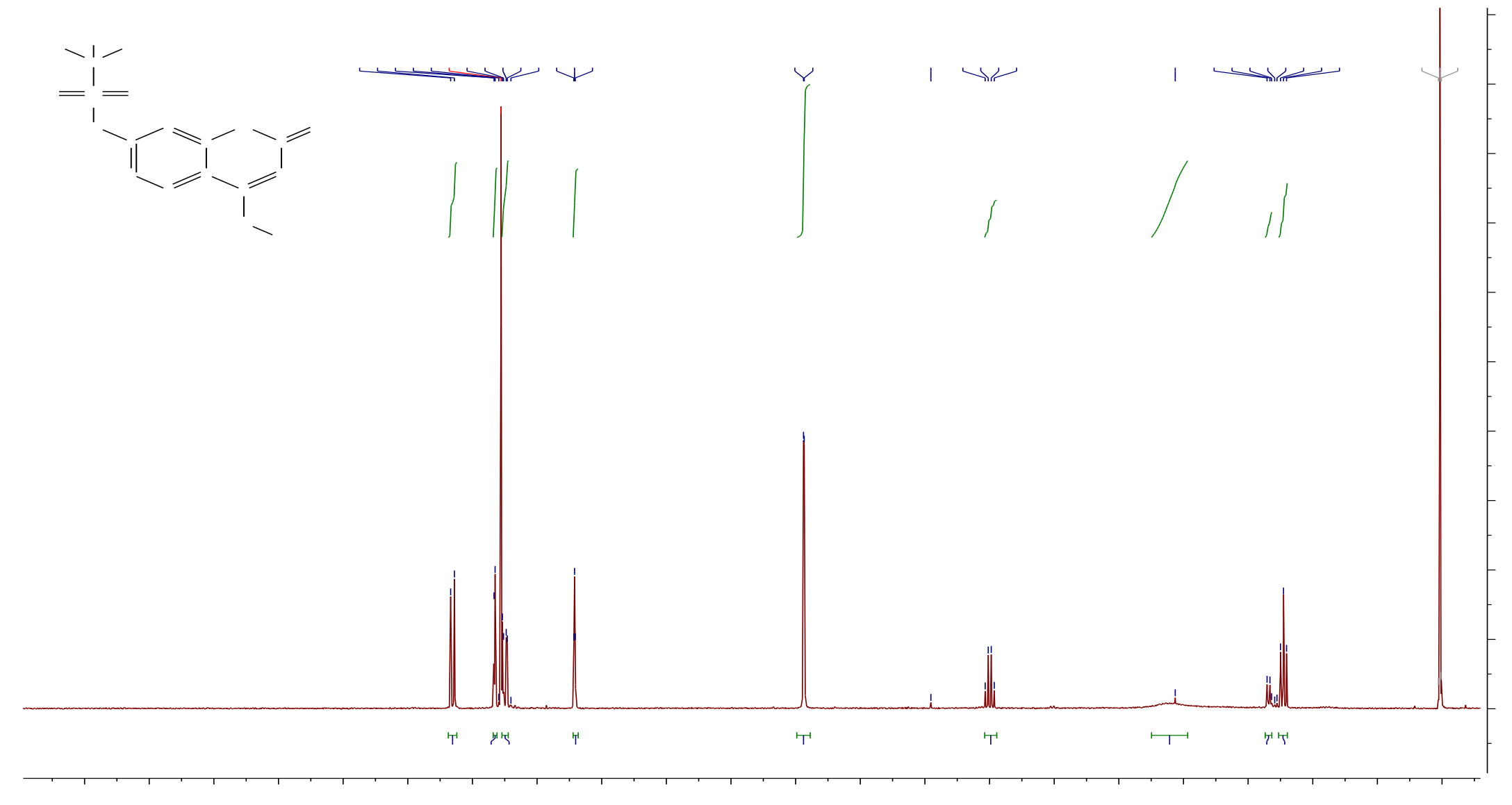

${ }^{1} \mathrm{H}$-NMR spectrum of 7-hydroxy-4-(hydroxymethyl)-2H-chromen-2-one 3.4 in $\mathrm{CDCl}_{3}$. 


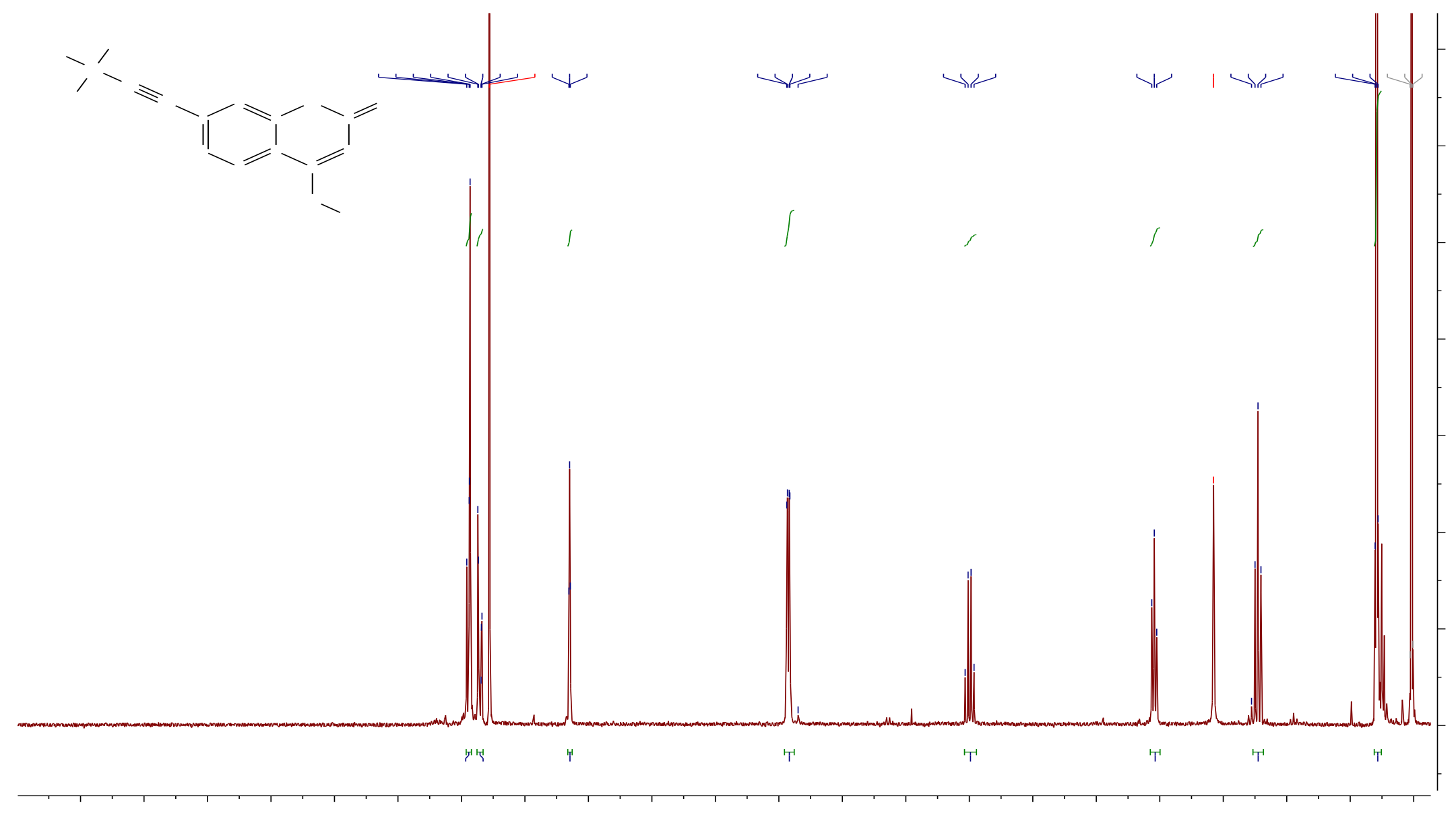

B.3 ${ }^{1} \mathrm{H}-\mathrm{NMR}$ spectrum of 4-(hydroxymethyl)-7-((trimethylsilyl)ethynyl)-2H-chromen-2-one 3.5 in $\mathrm{CDCl}_{3}$. 


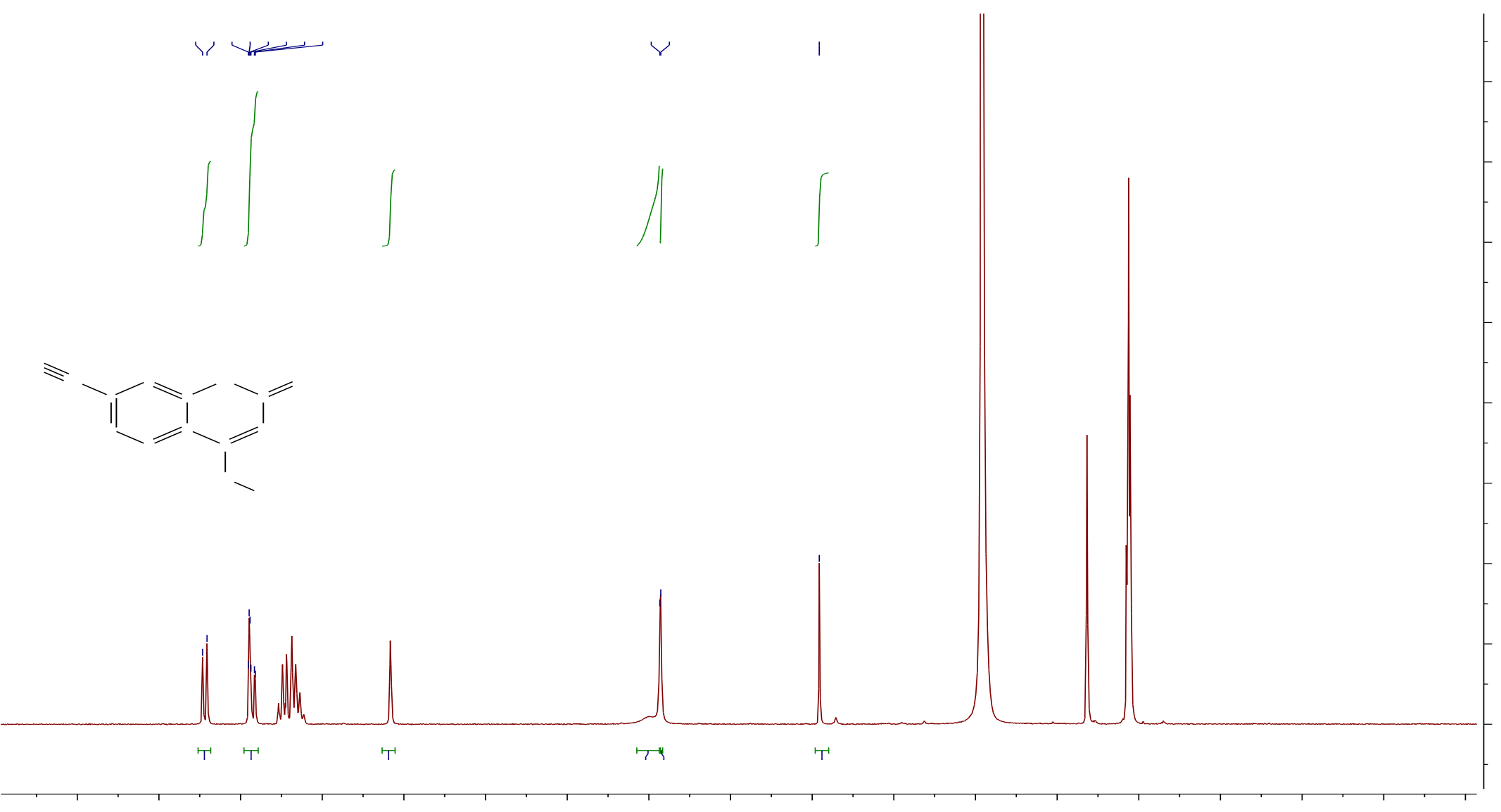

B.4 ${ }^{1} \mathrm{H}-\mathrm{NMR}$ spectrum of 7-ethynyl-4-(hydroxymethyl)-2H-chromen-2-one 3.6 in d6-DMSO. 


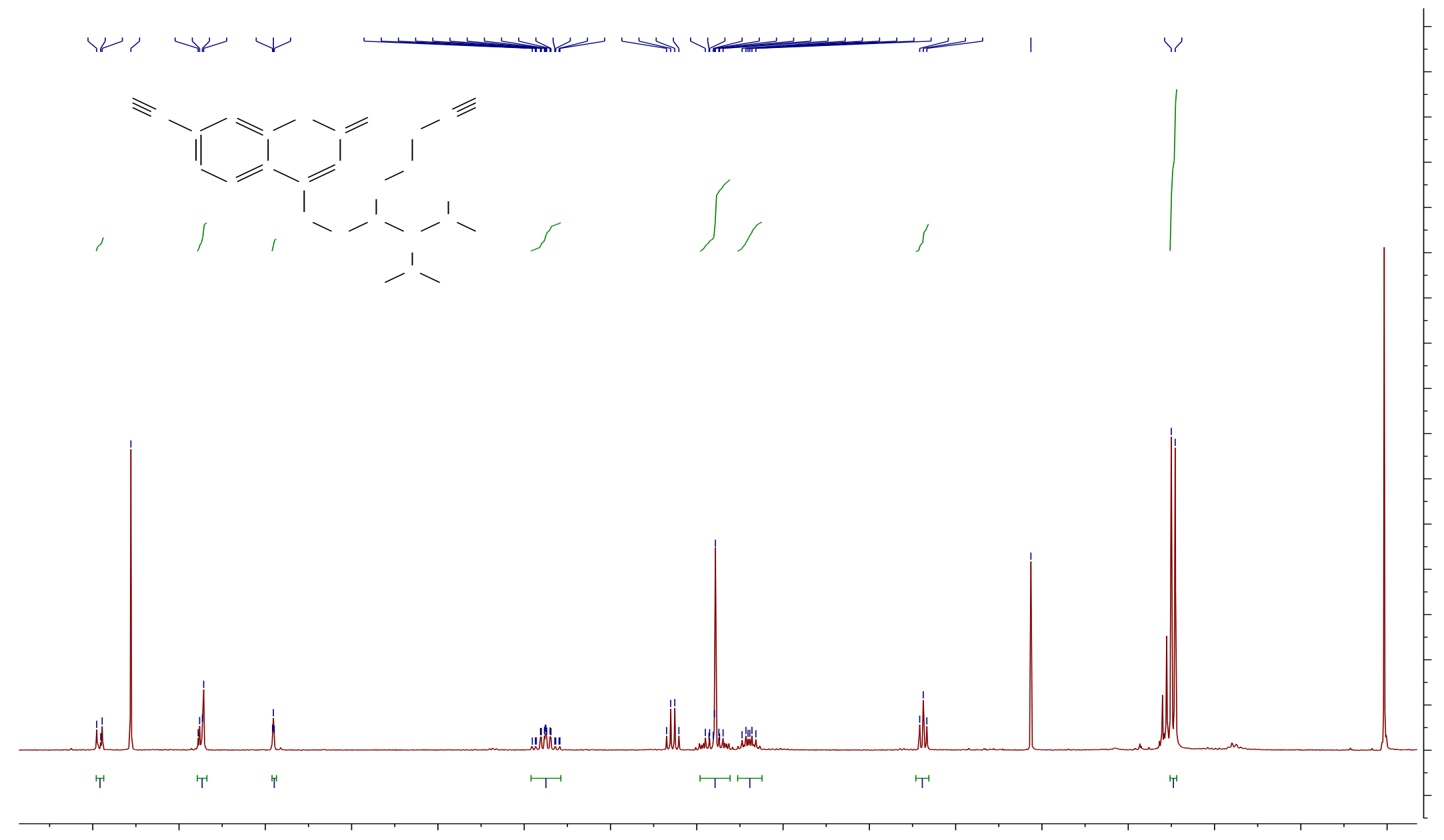

${ }^{1} \mathrm{H}-\mathrm{NMR}$ spectrum of 2-cyanoethyl ((7-ethynyl-2-oxo-2H-chromen-4-yl)methyl) diisopropylphosphoramidite 3.7 in $\mathrm{CDCl}_{3}$. 


\section{Appendix C Mass spectra for thrombin sensors}

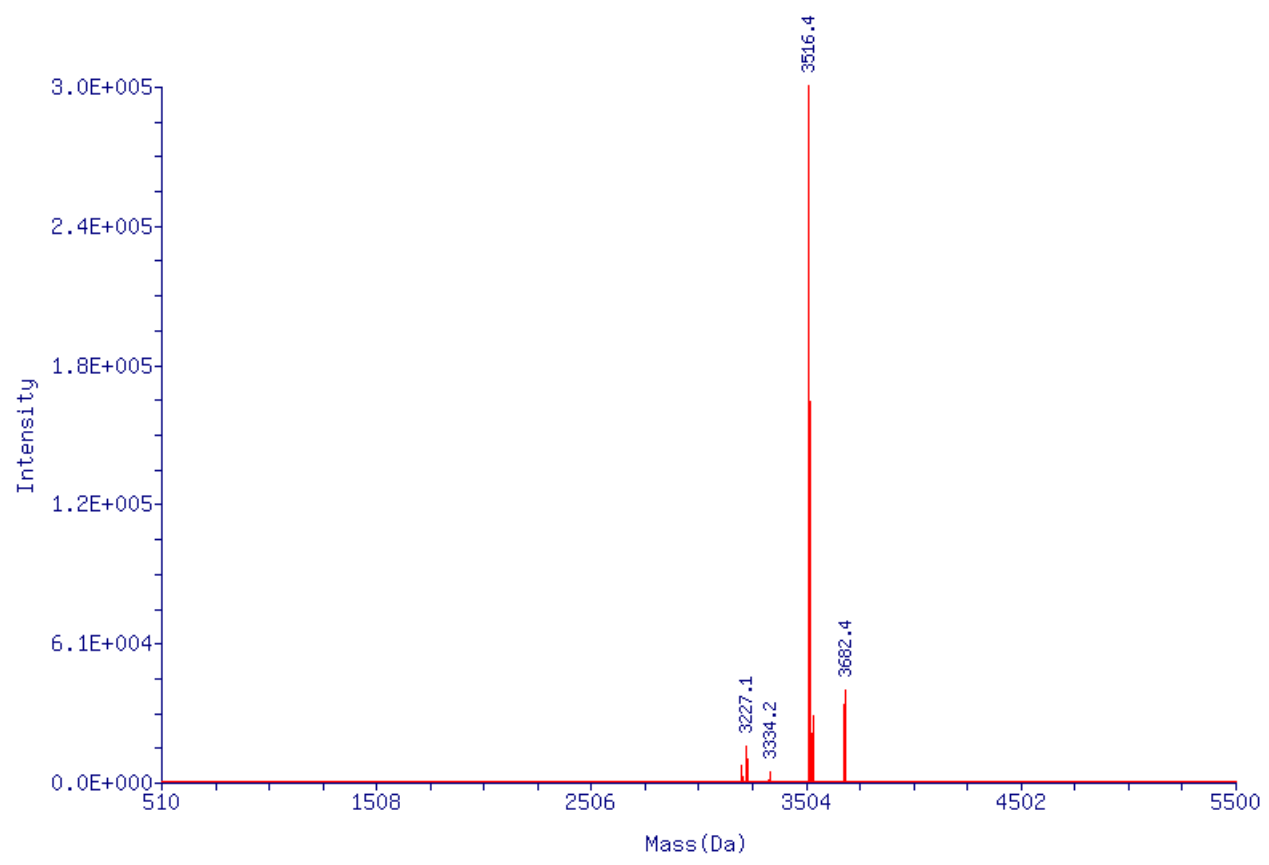

C.1 Deconvoluted ESI-MS of the 5'-coumarin SRA_2 probe.

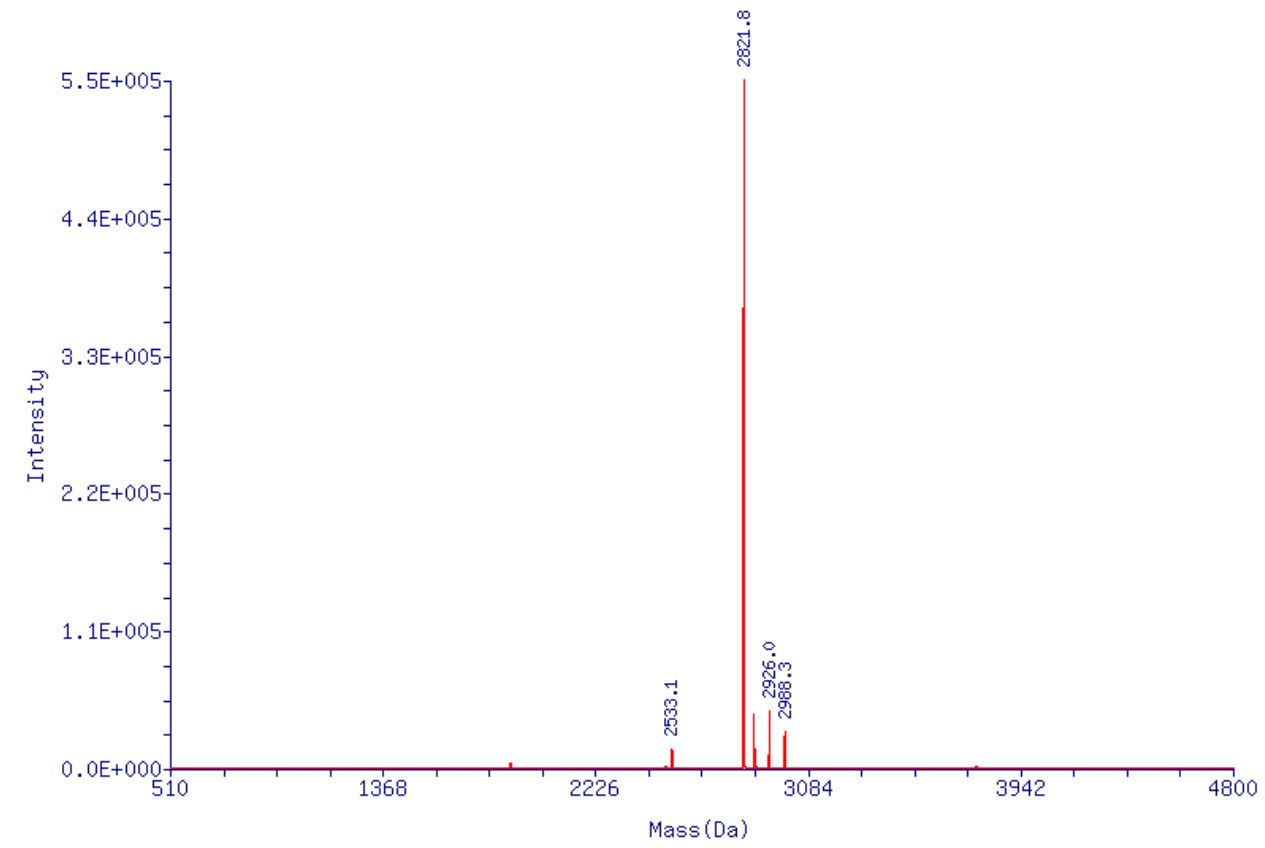

C.2 Deconvoluted ESI-MS of the 3'-amine SRA_2 probe. 


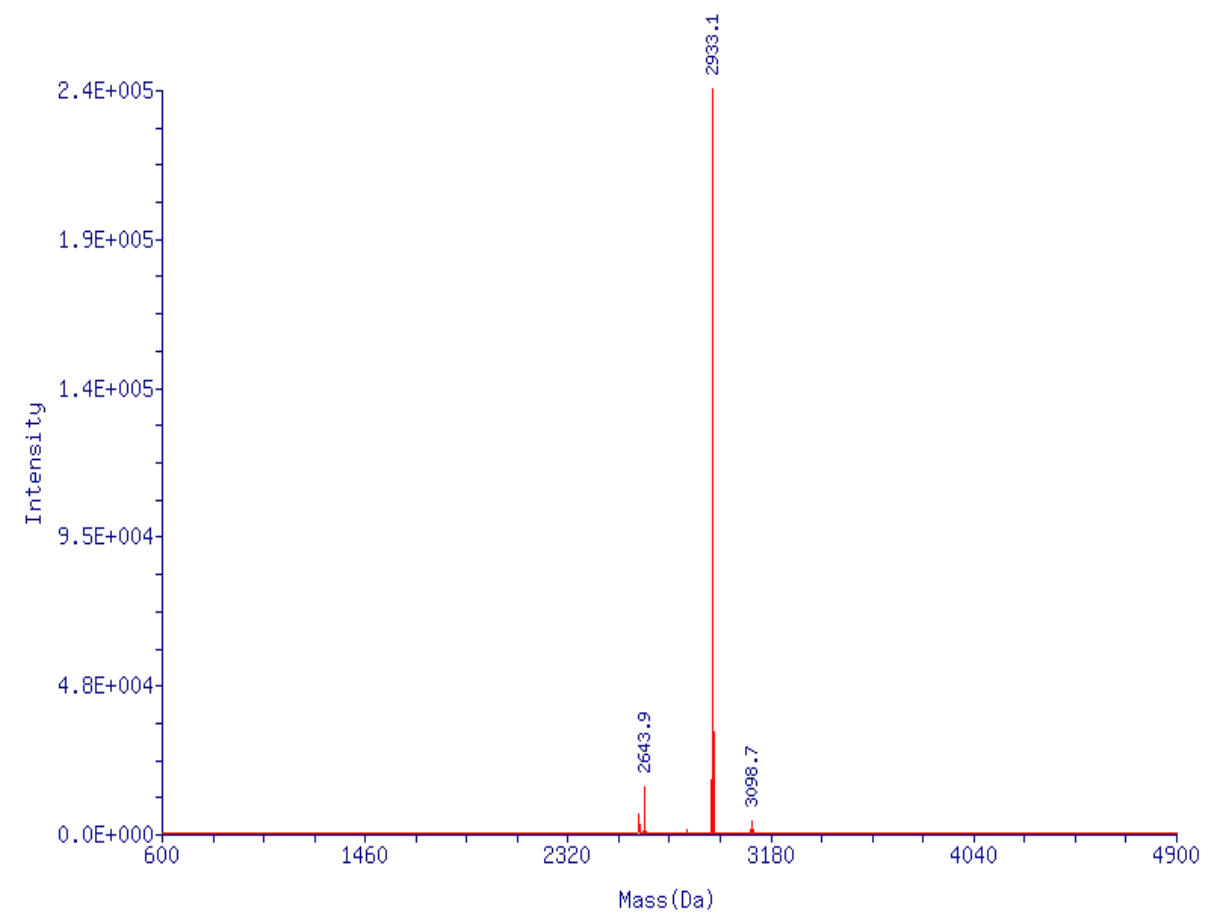

C.3 Deconvoluted ESI-MS of the 3'-azide SRA_2 probe

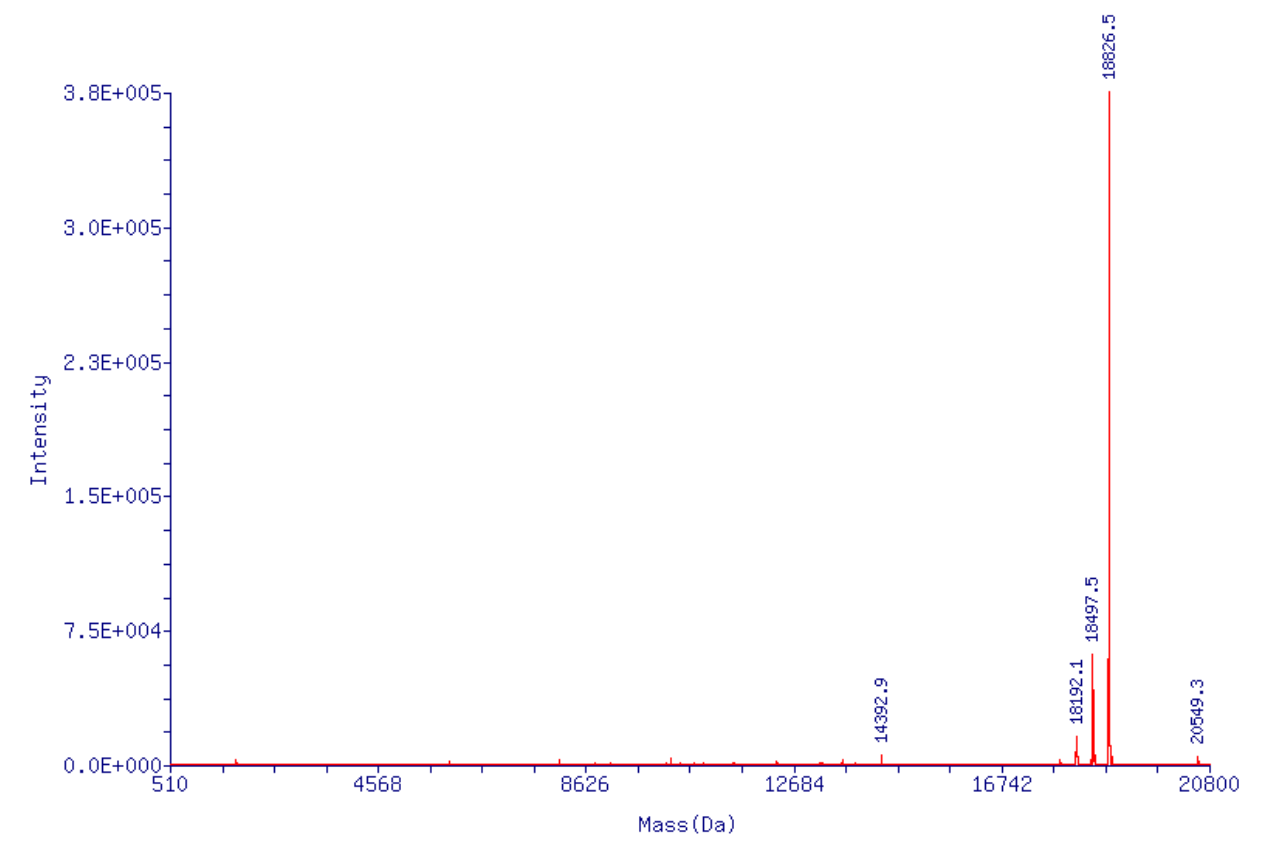

\section{C.4 Deconvoluted ESI-MS of the SRA_2 aptamer}




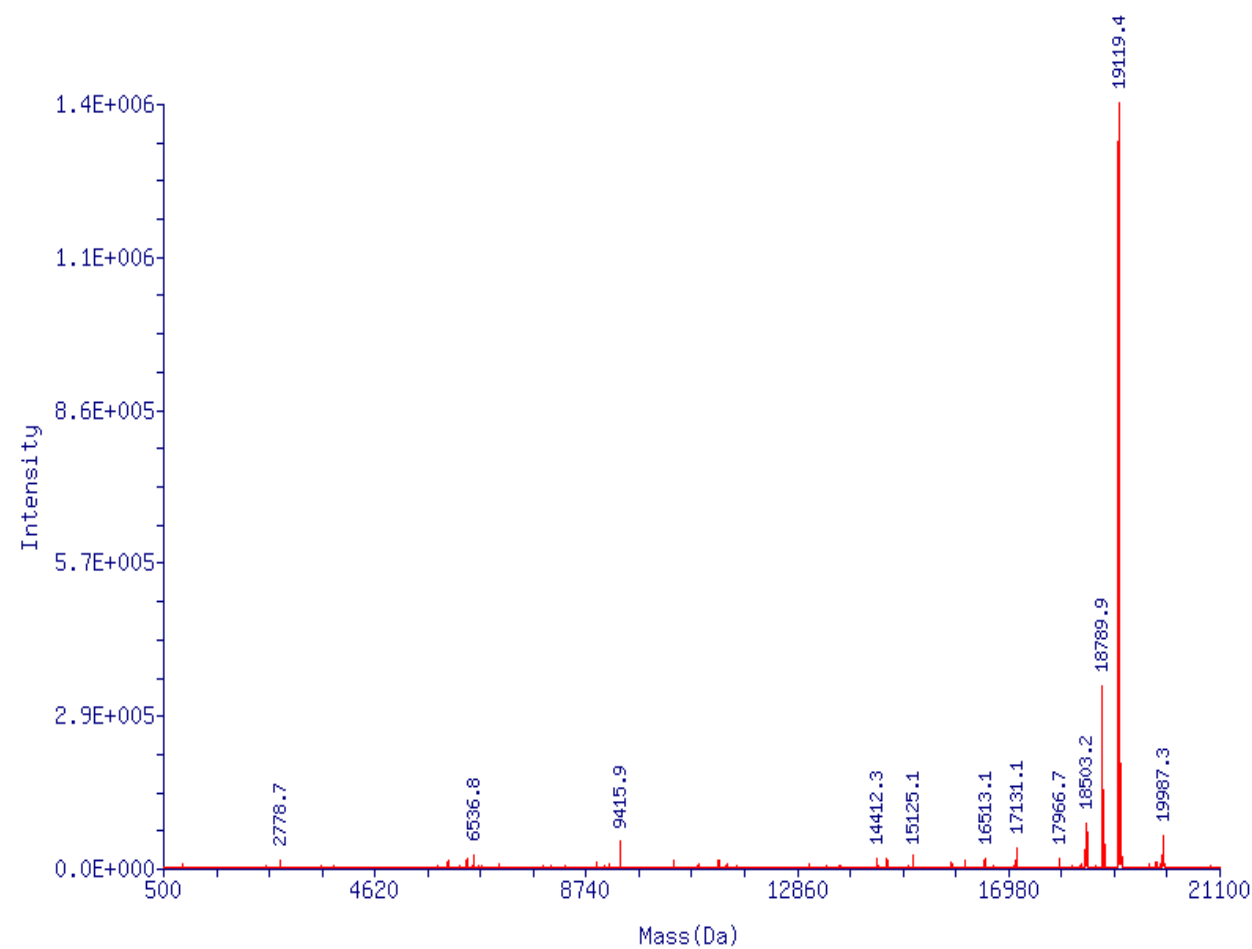

C.5 Deconvoluted ESI-MS of the single aptamer system labeled with the azide and the amine modifiers.

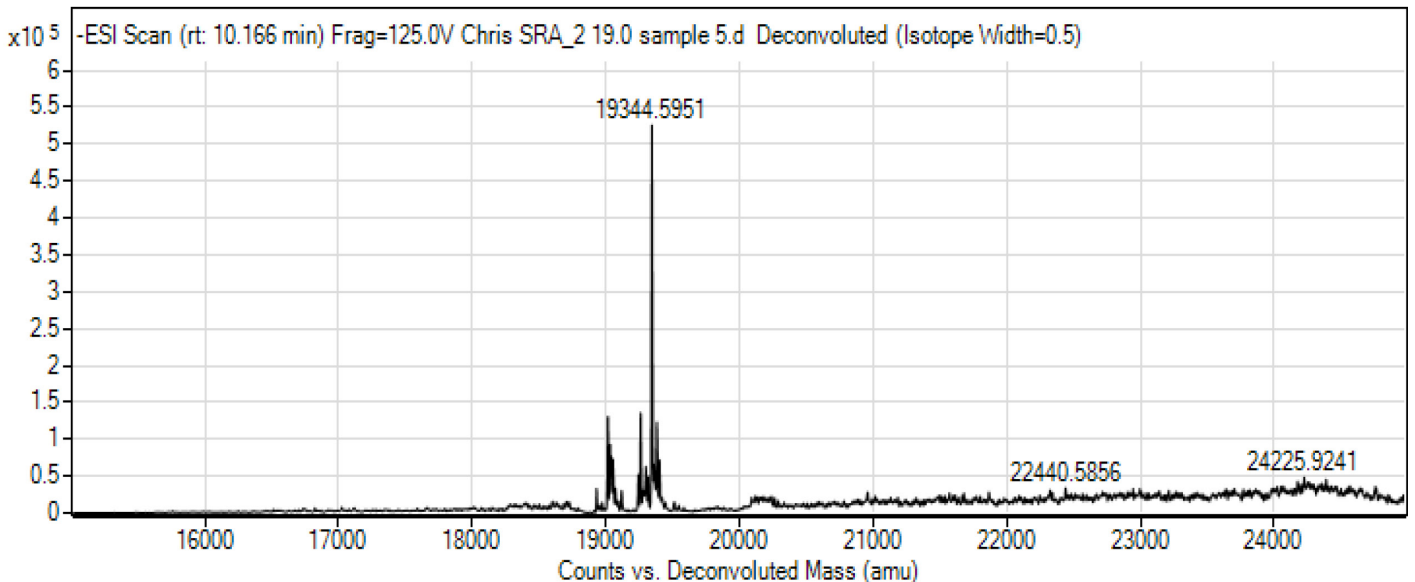

C.6 Deconvoluted ESI-MS of the single aptamer system labeled with the azide and the coumarin modifiers. 


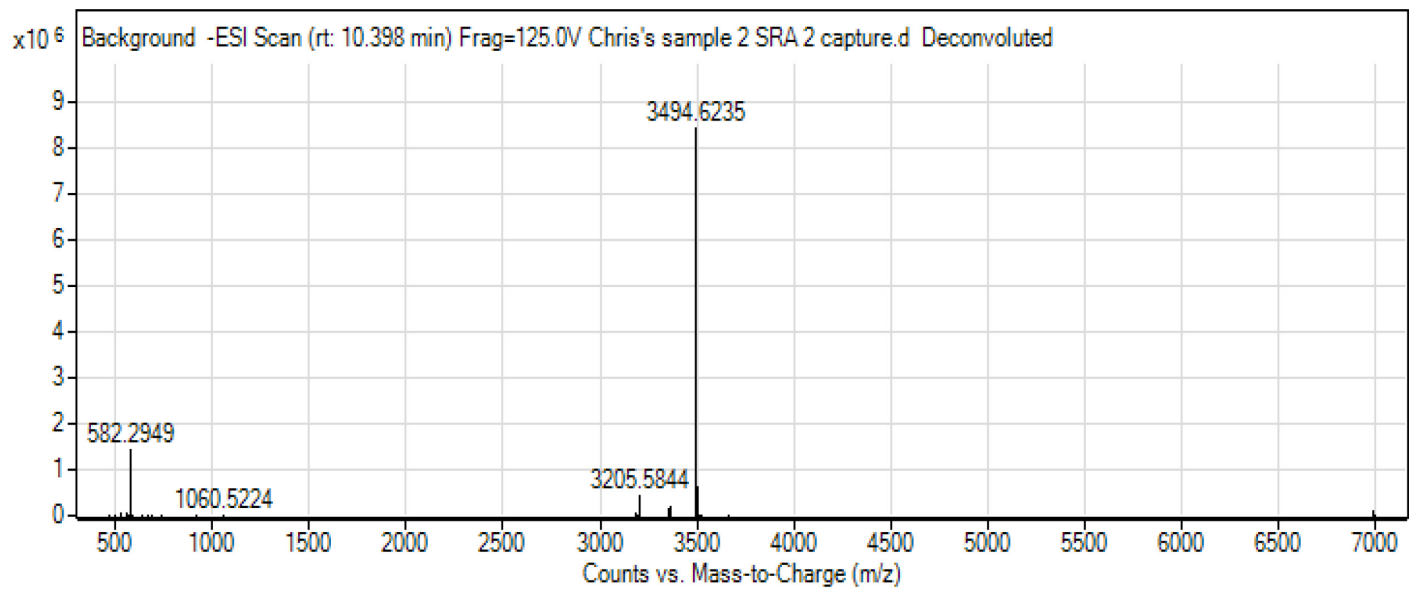

C.7 Deconvoluted ESI-MS of the SRA_2 capture probe. 


\section{Appendix D Mass spectra for cocaine sensor}

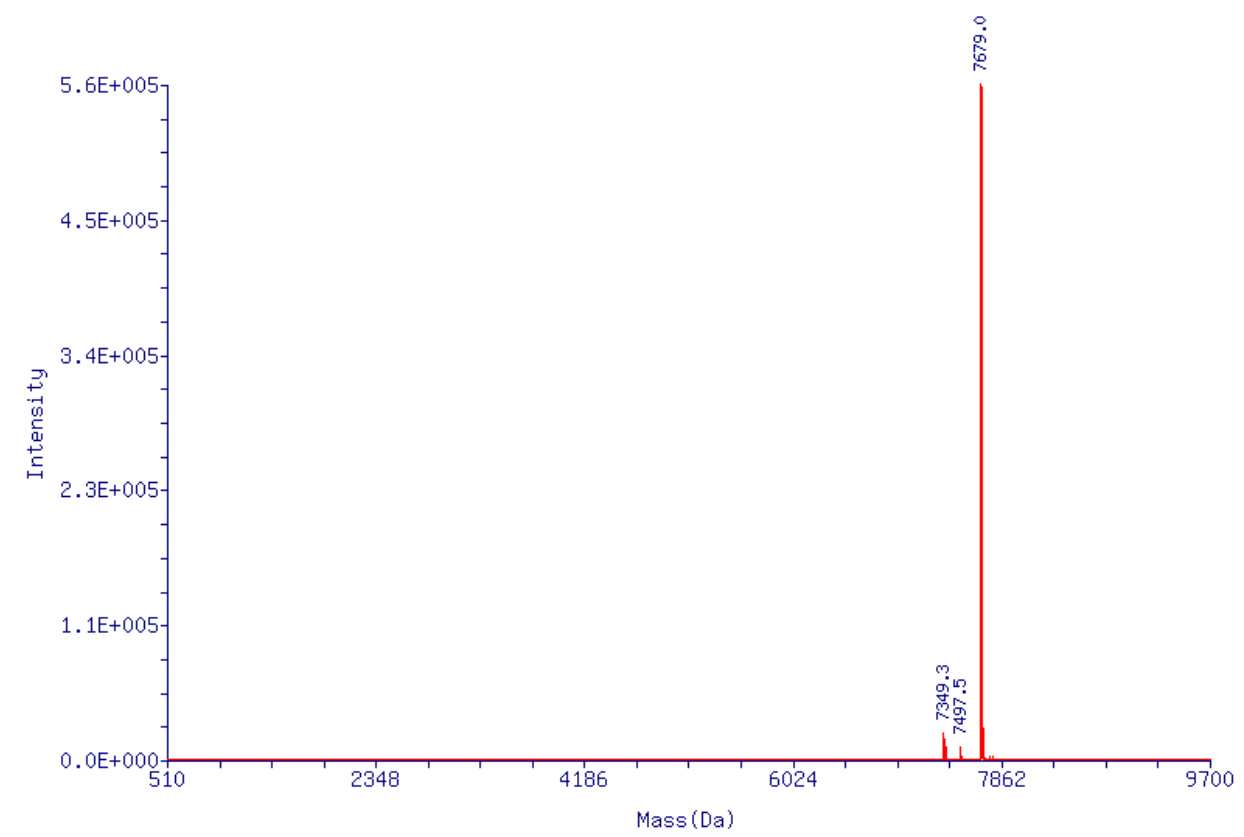

D.1 Deconvoluted mass spectrum of the alkyne-coumarin modified cocaine split aptamer, confirming its expected molecular weight of $7680 \mathrm{~g} / \mathrm{mol}$. 


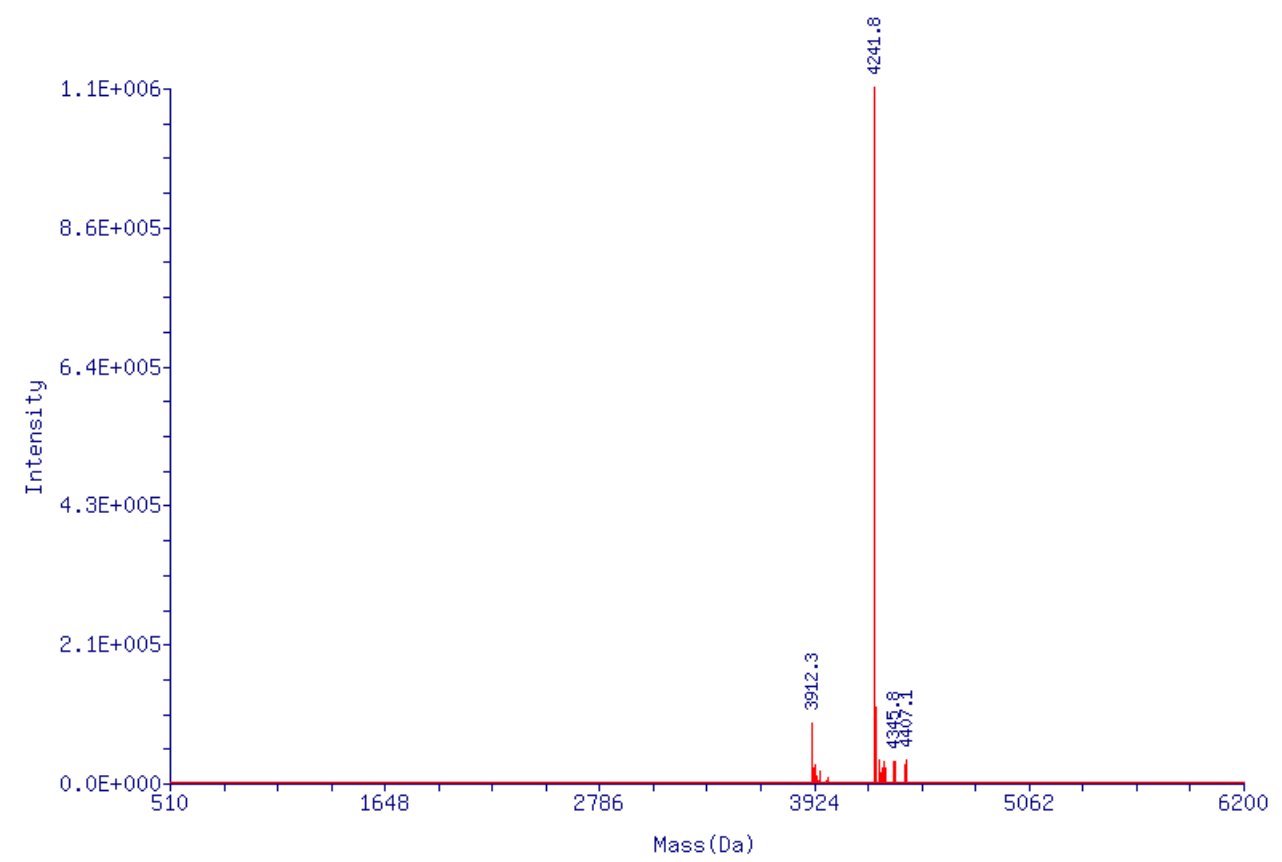

D.2 Deconvoluted mass spectrum of the amine modified cocaine split aptamer, confirming its expected molecular weight of $4242 \mathrm{~g} / \mathrm{mol}$.

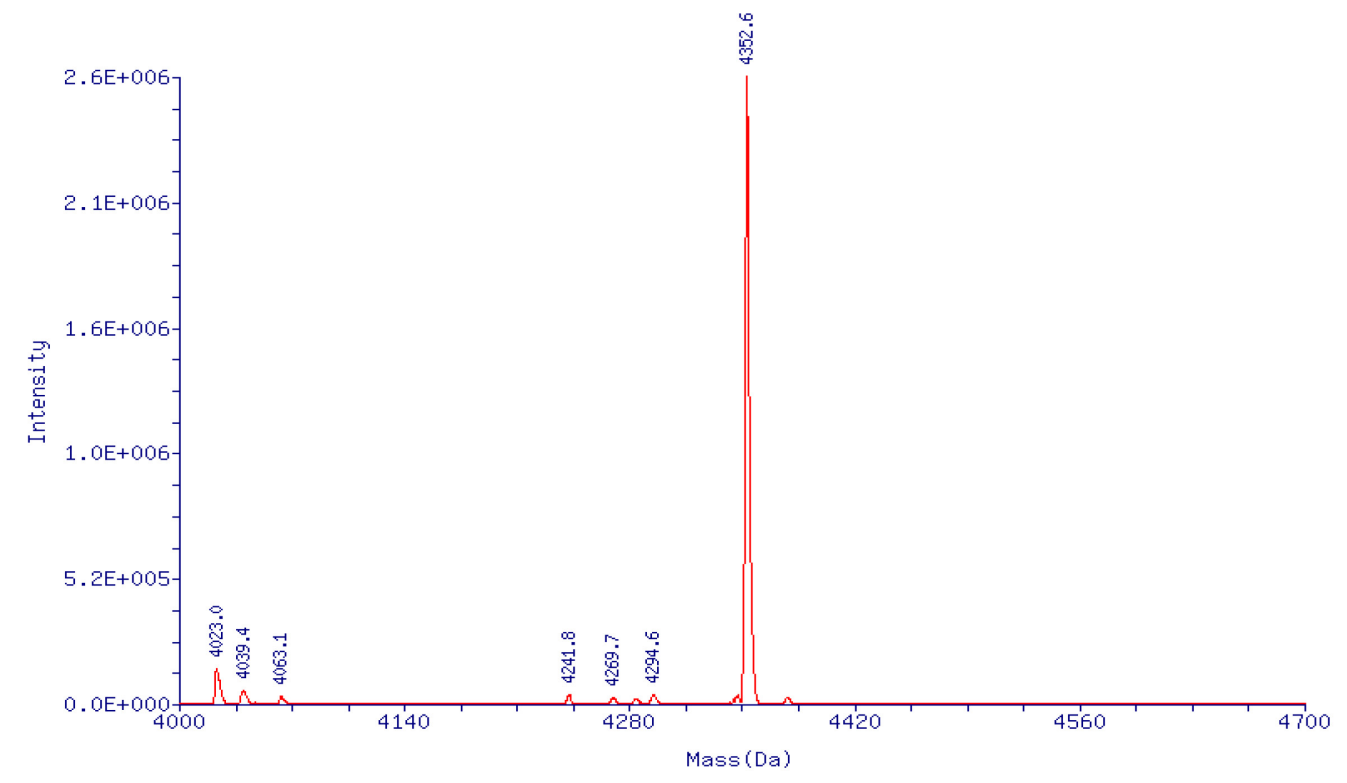

D.3 Deconvoluted mass spectrum of azide modified cocaine coumarin split aptamer. 


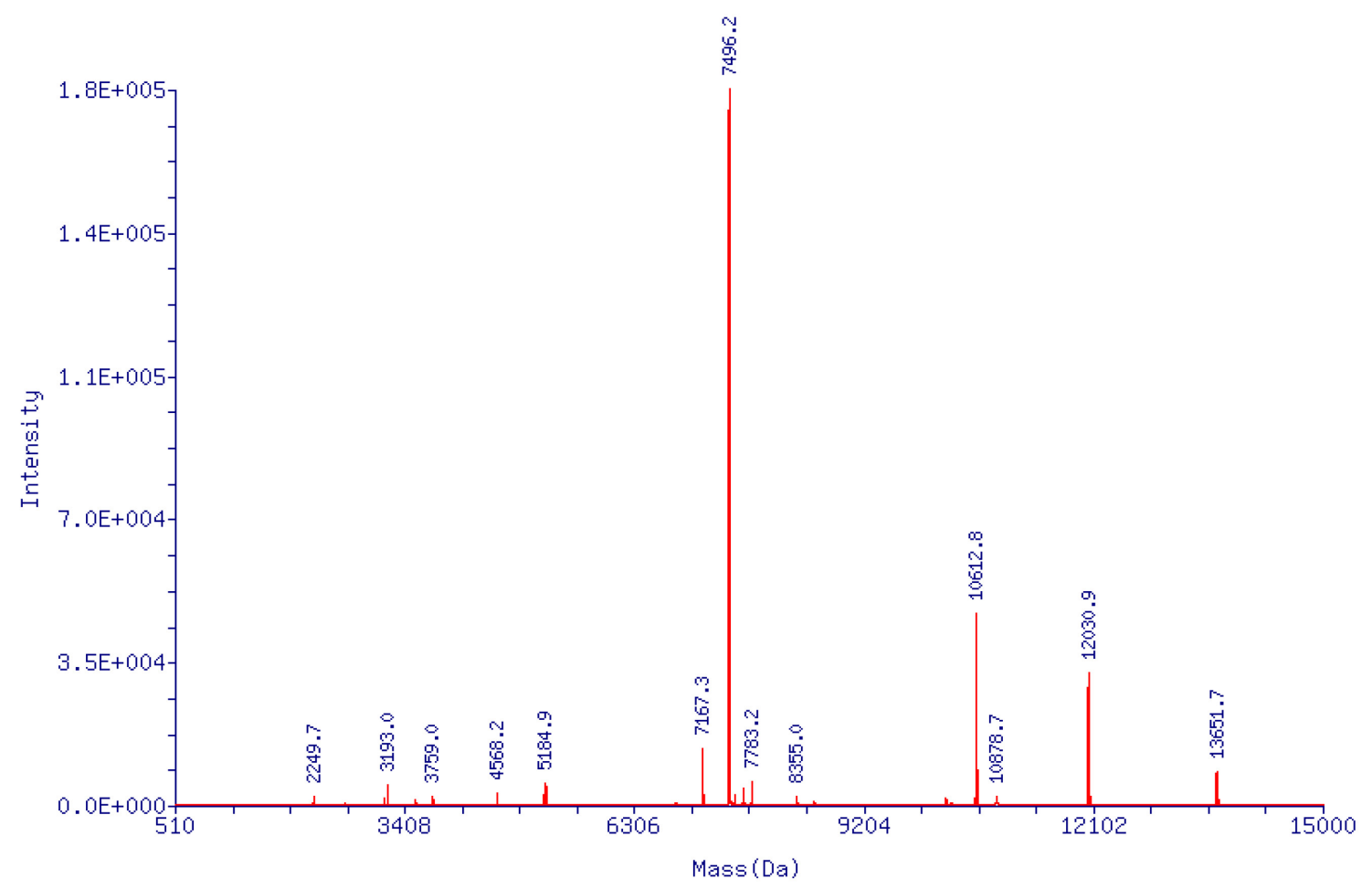

D.4 Deconvoluted mass spectrum of the cocaine ligation product, with the expected molecular weight of the ligated product being 12030.9 . 


\section{References}

1. Lu, Y.; Liu, J., Curr. Opin. Biotechnol. 2006, 17 (6), 580-588.

2. Mattice, C. M.; DeRosa, M. C., Biodrugs 2015, 29 (3), 151-65.

3. Seeman, N. C., J. Theor. Biol. 1982, 99 (2), 237-247.

4. Seeman, N. C.; Sleiman, H. F., Nat. Rev. Mater. 2018, 3 (1), 17068.

5. Nummelin, S.; Kommeri, J.; Kostiainen Mauri, A.; Linko, V., Adv. Mater. 2018, 30 (24), 1703721.

6. Kohman Richie, E.; Kunjapur Aditya, M.; Hysolli, E.; Wang, Y.; Church George, M., Angew. Chem. Int. Ed. 2018, 57 (16), 4313-4328.

7. Bathe, M.; Rothemund, P. W. K., MRS Bull. 2017, 42 (12), 882-888.

8. Peng, H.; Newbigging, A. M.; Wang, Z.; Tao, J.; Deng, W.; Le, X. C.; Zhang, H., Anal. Chem. 2018, 90 (1), 190-207.

9. Silverman, S. K., Chem. Commun. 2008, (30), 3467-3485.

10. Silverman, S. K., Artificial Functional Nucleic Acids: Aptamers, Ribozymes, and

Deoxyribozymes Identified by In Vitro Selection. In Functional Nucleic Acids for

Analytical Applications, Yingfu, L.; Yi, L., Eds. Springer New York: New York, NY, 2009; pp 47-108.

11. Silverman, S. K., Trends Biochem. Sci. 2016, 41 (7), 595-609.

12. Silverman, S. K., Acc. Chem. Res. 2009, 42 (10), 1521-1531.

13. Banerjee, J.; Nilsen-Hamilton, M., J. Mol. Med. (Berl.) 2013, 91 (12), 1333-42.

14. Ellington, A. D.; Szostak, J. W., Nature 1990, 346 (6287), 818-22.

15. McKeague, M.; Derosa, M. C., J. Nucleic Acids 2012, 2012, 748913.

16. Tuerk, C.; Gold, L., Science 1990, 249 (4968), 505-10.

17. Robertson, D. L.; Joyce, G. F., Nature 1990, 344 (6265), 467-468.

18. Song, S.; Wang, L.; Li, J.; Fan, C.; Zhao, J., TrAC, Trends Anal. Chem. 2008, 27 (2), 108-117.

19. Mayer, G.; Jenne, A., Biodrugs 2004, 18 (6), 351-359.

20. Shangguan, D.; Li, Y.; Tang, Z.; Cao, Z. C.; Chen, H. W.; Mallikaratchy, P.; Sefah,

K.; Yang, C. J.; Tan, W., Proc. Natl. Acad. Sci. U. S. A. 2006, 103 (32), 11838-11843.

21. Fang, X.; Tan, W., Acc. Chem. Res. 2009, 43 (1), 48-57.

22. Sefah, K.; Shangguan, D.; Xiong, X.; O'Donoghue, M. B.; Tan, W., Nat. Protocols 2010, $5(6), 1169-1185$.

23. Ye, M.; Hu, J.; Peng, M.; Liu, J.; Liu, J.; Liu, H.; Zhao, X.; Tan, W., Int. J. Mol. Sci. 2012, 13 (3), 3341-3353.

24. Cheng, C.; Chen, Y. H.; Lennox, K. A.; Behlke, M. A.; Davidson, B. L., Mol. Ther. Nucleic Acids 2013, 2, e67.

25. Hermann, T.; Patel, D. J., Science 2000, 287 (5454), 820-5.

26. Jayasena, S. D., Clin. Chem. 1999, 45 (9), 1628-1650.

27. Mirkin, S. M., Front. Biosci. 2008, 13, 1064-71.

28. Kypr, J.; Kejnovská, I.; Renčiuk, D.; Vorlíčková, M., Nucleic Acids Res. 2009, 37 (6), 1713-1725.

29. Keefe, A. D.; Pai, S.; Ellington, A., Nat. Rev. Drug Discov. 2010, 9 (7), 537-550. 
30. Tolle, F.; Mayer, G., Chem. Sci. 2013, 4 (1), 60-67.

31. Oney, S.; Lam, R. T. S.; Bompiani, K. M.; Blake, C. M.; Quick, G.; Heidel, J. D.;

Liu, J. Y.-C.; Mack, B. C.; Davis, M. E.; Leong, K. W.; Sullenger, B. A., Nat. Med. 2009, 15 (10), 1224-1228.

32. Rusconi, C. P.; Roberts, J. D.; Pitoc, G. A.; Nimjee, S. M.; White, R. R.; Quick Jr, G.;

Scardino, E.; Fay, W. P.; Sullenger, B. A., Nat Biotech 2004, 22 (11), 1423-1428.

33. Baldrich, E.; Restrepo, A.; O'Sullivan, C. K., Anal. Chem. 2004, 76 (23), 7053-7063.

34. Cho, E. J.; Lee, J.-W.; Ellington, A. D., Annu. Rev. Anal. Chem. 2009, 2 (1), 241-264.

35. Wang, R. E.; Zhang, Y.; Cai, J.; Cai, W.; Gao, T., Curr. Med. Chem. 2011, 18 (27),

4175-4184.

36. Zhou, W.; Jimmy Huang, P.-J.; Ding, J.; Liu, J., Analyst 2014, 139 (11), 2627-2640.

37. Xing, H.; Hwang, K.; Li, J.; Torabi, S.-F.; Lu, Y., Curr. Opin. Chem. Eng. 2014, 4, 79-87.

38. Levy-Nissenbaum, E.; Radovic-Moreno, A. F.; Wang, A. Z.; Langer, R.; Farokhzad,

O. C., Trends Biotechnol. 2008, 26 (8), 442-449.

39. Gatto, B.; Palumbo, M.; Sissi, C., Curr. Med. Chem. 2009, 16 (10), 1248-1265.

40. Gragoudas, E. S.; Adamis, A. P.; Cunningham, E. T.; Feinsod, M.; Guyer, D. R., New Engl. J. Med. 2004, 351 (27), 2805-2816.

41. Ng, E. W. M.; Shima, D. T.; Calias, P.; Cunningham, E. T.; Guyer, D. R.; Adamis, A. P., Nat. Rev. Drug Discov. 2006, 5 (2), 123-132.

42. Silverman, S., Artificial Functional Nucleic Acids: Aptamers, Ribozymes, and Deoxyribozymes Identified by In Vitro Selection. In Functional Nucleic Acids for Analytical Applications, Yingfu, L.; Yi, L., Eds. Springer New York: 2009; pp 47-108. 43. Ku, T.-H.; Zhang, T.; Luo, H.; Yen, T. M.; Chen, P.-W.; Han, Y.; Lo, Y.-H., Sensors 2015, 15 (7), 16281-16313.

44. Zhang, Y.; Chen, Y.; Han, D.; Ocsoy, I.; Tan, W., Bioanalysis 2010, 2 (5), 907-918. 45. Stoltenburg, R.; Nikolaus, N.; Strehlitz, B., J. Anal. Methods Chem. 2012, 2012, 415697.

46. Oh, S. S.; Plakos, K.; Lou, X.; Xiao, Y.; Soh, H. T., Proc. Natl. Acad. Sci. U.S.A. 2010, 107 (32), 14053-14058.

47. Reese, C. B., Org. Biomol. Chem. 2005, 3 (21), 3851-3868.

48. Khorana, H. G.; Razzell, W. E.; Gilham, P. T.; Tener, G. M.; Pol, E. H., J. Am. Chem. Soc. 1957, 79 (4), 1002-1003.

49. Gilham, P. T.; Khorana, H. G., J. Am. Chem. Soc. 1958, 80 (23), 6212-6222.

50. Agarwal, K. L.; Yamazaki, A.; Cashion, P. J.; Khorana, H. G., Angew. Chem. Int. Ed. 1972, $11(6), 451-459$.

51. Schaller, H.; Weimann, G.; Lerch, B.; Khorana, H. G., J. Am. Chem. Soc. 1963, 85

(23), 3821-3827.

52. Letsinger, R. L.; Mahadevan, V., J. Am. Chem. Soc. 1965, 87 (15), 3526-3527.

53. Letsinger, R. L.; Ogilvie, K. K., J. Am. Chem. Soc. 1967, 89 (18), 4801-4803.

54. Letsinger, R. L.; Ogilvie, K. K.; Miller, P. S., J. Am. Chem. Soc. 1969, 91 (12), 33603365 .

55. Letsinger, R. L.; Lunsford, W. B., J. Am. Chem. Soc. 1976, 98 (12), 3655-3661. 
56. Beaucage, S. L.; Caruthers, M. H., Tetrahedron Lett. 1981, 22 (20), 1859-1862.

57. Abramova, T., Molecules 2013, 18 (1).

58. Percivalle, C.; Bartolo, J.-F.; Ladame, S., Org. Biomol. Chem. 2013, 11 (1), 16-26.

59. Rosen, C. B.; Toerring, T.; Gothelf, K. V., Nucleic Acids Mol. Biol. 2014, 29 (Nucleic Acid Nanotechnology), 173-197.

60. Michaelis, J.; Roloff, A.; Seitz, O., Org. Biomol. Chem. 2014, 12 (18), 2821-2833.

61. Herrlein, M. K.; Letsinger, R. L., Nucleic Acids Res. 1994, 22 (23), 5076-5078.

62. Herrlein, M. K.; Nelson, J. S.; Letsinger, R. L., J. Am. Chem. Soc. 1995, 117 (40),

10151-10152.

63. Xu, Y.; Kool, E. T., Tetrahedron Lett. 1997, 38 (32), 5595-5598.

64. Xu, Y.; Kool, E. T., Nucleic Acids Res. 1999, 27 (3), 875-881.

65. Xu, Y.; Kool, E. T., J. Am. Chem. Soc. 2000, 122 (37), 9040-9041.

66. Sando, S.; Kool, E. T., J. Am. Chem. Soc. 2002, 124 (10), 2096-2097.

67. Sando, S.; Abe, H.; Kool, E. T., J. Am. Chem. Soc. 2004, 126 (4), 1081-1087.

68. Li, X.; Zhan, Z.-Y. J.; Knipe, R.; Lynn, D. G., J. Am. Chem. Soc. 2002, 124 (5), 746747.

69. Gartner Zev, J.; Kanan Matthew, W.; Liu David, R., Angew. Chem. Int. Ed. 2002, 41 (10), 1796-1800.

70. Gorska, K.; Winssinger, N., Angew. Chem., Int. Ed. 2013, 52 (27), 6820-6843.

71. Gartner, Z. J.; Kanan, M. W.; Liu, D. R., Angew. Chem. Int. Ed. 2002, 41 (10), 17961800.

72. Yang, H.; Seela, F., Chem. Eur. J. 2017, 23 (14), 3375-3385.

73. Mukae, M.; Ihara, T.; Tabara, M.; Arslan, P.; Jyo, A., Supramol. Chem. 2009, 21 (3-

4), 292-295.

74. Sakurai, K.; Snyder, T. M.; Liu, D. R., J. Am. Chem. Soc. 2005, 127 (6), 1660-1661.

75. Saxon, E.; Bertozzi, C. R., Science 2000, 287 (5460), 2007.

76. Cai, J.; Li, X.; Yue, X.; Taylor, J. S., J. Am. Chem. Soc. 2004, 126 (50), $16324-$ 16325.

77. Pianowski, Z. L.; Winssinger, N., Chem. Commun. 2007, (37), 3820-3822.

78. Franzini Raphael, M.; Kool Eric, T., ChemBioChem 2008, 9 (18), 2981-2988.

79. Franzini, R. M.; Kool, E. T., J. Am. Chem. Soc. 2009, 131 (44), 16021-16023.

80. Cló, E.; Snyder, J. W.; Voigt, N. V.; Ogilby, P. R.; Gothelf, K. V., J. Am. Chem. Soc. 2006, 128 (13), 4200-4201.

81. Han, K.; Liang, Z.; Zhou, N., Sensors 2010, 10 (5), 4541-4557.

82. Han, K.; Zhu, J.-Y.; Wang, S.-B.; Li, Z.-H.; Cheng, S.-X.; Zhang, X.-Z., J. Mater.

Chem. B 2015, 3 (41), 8065-8069.

83. Seok Kim, Y.; Ahmad Raston, N. H.; Bock Gu, M., Biosensors Bioelectron. 2016, 76, 2-19.

84. Ensafi, A. A.; Amini, M.; Rezaei, B., Microchim. Acta 2018, 185 (5), 1-7.

85. Hianik, T.; Ostatná, V.; Sonlajtnerova, M.; Grman, I., Bioelectrochemistry 2007, 70

(1), 127-133.

86. Li, F.; Yu, Z.; Han, X.; Shi, W.; Liu, Y.; Yan, H.; Zhang, G., Sens. Actuators, B 2018, $273,480-487$. 
87. Zhang, Y.; Xia, J.; Zhang, F.; Wang, Z.; Liu, Q., Sens. Actuators, B 2018, 267, 412418.

88. Zhu, X.; Kou, F.; Xu, H.; Han, Y.; Yang, G.; Huang, X.; Chen, W.; Chi, Y.; Lin, Z., Sens. Actuators, B 2018, 270, 263-269.

89. Kitajima, M.; Wang, N.; Tay, M. Q. X.; Miao, J.; Whittle, A. J., Micro Nano Lett.

2016, 11 (10), 582-585.

90. Babelova, L.; Sohova, M. E.; Poturnayova, A.; Burikova, M.; Bizik, J.; Hianik, T., Electroanalysis 2018, Ahead of Print.

91. Yu, X.; Chen, F.; Wang, R.; Li, Y., J. Biotechnol. 2018, 266, 39-49.

92. Kim, Y. H.; Kim, J. P.; Han, S. J.; Sim, S. J., Sensors Actuators B: Chem. 2009, 139 (2), 471-475.

93. Bai, Y.; Feng, F.; Zhao, L.; Wang, C.; Wang, H.; Tian, M.; Qin, J.; Duan, Y.; He, X., Biosens. Bioelectron. 2013, 47, 265-70.

94. Fukusaki, E.-i.; Hasunuma, T.; Kajiyama, S.-i.; Okazawa, A.; Itoh, T. J.; Kobayashi, A., Bioorg. Med. Chem. Lett. 2001, 11 (22), 2927-2930.

95. Hawkins, M. E., Cell Biochem. Biophys. 2001, 34 (2), 257-281.

96. Fang, X.; Cao, Z.; Beck, T.; Tan, W., Anal. Chem. 2001, 73 (23), 5752-5757.

97. Tyagi, S.; Kramer, F. R., Nat. Biotechnol. 1996, 14, 303.

98. Mirkin, C. A.; Letsinger, R. L.; Mucic, R. C.; Storhoff, J. J., Nature 1996, 382, 607.

99. Xu, Y.; Karalkar, N. B.; Kool, E. T., Nat. Biotech. 2001, 19 (2), 148-152.

100. Silverman, A. P.; Kool, E. T., Trends Biotechnol. 2005, 23 (5), 225-230.

101. Silverman, A. P.; Kool, E. T., Nucleic Acids Res. 2005, 33 (15), 4978-4986.

102. Moter, A.; Göbel, U. B., J. Microbiol. Methods 2000, 41 (2), 85-112.

103. Sando, S.; Kool, E. T., J. Am. Chem. Soc. 2002, 124 (10), 2096-7.

104. Abe, H.; Kool, E. T., J. Am. Chem. Soc. 2004, 126 (43), 13980-13986.

105. Miller, G. P.; Silverman, A. P.; Kool, E. T., Biorg. Med. Chem. 2008, 16 (1), 56-64.

106. Kleinbaum, D. J.; Miller, G. P.; Kool, E. T., Bioconjugate Chem. 2010, 21 (6), 1115-1120.

107. Hussein, H. S.; Brasel, J. M., Toxicology 2001, 167 (2), 101-134.

108. Bennett, J. W.; Klich, M., Clin. Microbiol. Rev. 2003, 16 (3), 497-516.

109. Miller, J. D.; Schaafsma, A. W.; Bhatnagar, D.; Bondy, G.; Carbone, I.; Harris, L. J.; Harrison, G.; Munkvold, G. P.; Oswald, I. P.; Pestka, J. J.; Sharpe, L.; Sumarah, M. W.; Tittlemier, S. A.; Zhou, T., World Mycotoxin J. 2014, 7 (1), 63-82.

110. Pitt, J. I.; Miller, J. D., J. Agric. Food Chem. 2017, 65 (33), 7021-7033.

111. Ji, C.; Fan, Y.; Zhao, L., Anim Nutr 2016, 2 (3), 127-133.

112. Cruz-Aguado, J. A.; Penner, G., J. Agric. Food Chem. 2008, 56 (22), 10456-10461.

113. el Khoury, A.; Atoui, A., Toxins (Basel) 2010, 2 (4), 461-493.

114. Pfohl-Leszkowicz, A.; Manderville Richard, A., Mol. Nutr. Food Res. 2006, 51 (1), 61-99.

115. Afsah-Hejri, L.; Jinap, S.; Mirhosseini, H., Food Control 2012, 23 (1), 113-119.

116. Rhouati, A.; Yang, C.; Hayat, A.; Marty, J.-L., Toxins (Basel) 2013, 5 (11), 19882008.

117. Cabañes, F. J.; Bragulat, M. R.; Castellá, G., Toxins (Basel) 2010, 2 (5), 1111-1120. 
118. Duan, N.; Wu, S.-j.; Wang, Z.-P., Chin. J. Anal. Chem. 2011, 39 (3), 300-304. 119. Barthelmebs, L.; Jonca, J.; Hayat, A.; Prieto-Simon, B.; Marty, J.-L., Food Control 2011, 22 (5), 737-743.

120. McKeague, M.; Velu, R.; Hill, K.; Bardóczy, V.; Mészáros, T.; DeRosa, C. M., Toxins (Basel) 2014, 6 (8).

121. Lv, Z.; Chen, A.; Liu, J.; Guan, Z.; Zhou, Y.; Xu, S.; Yang, S.; Li, C., PLoS One 2014, 9 (1), e85968/1-e85968/5, 5 pp.

122. Zhao, Q.; Geng, X.; Wang, H., Anal. Bioanal. Chem. 2013, 405 (19), 6281-6286.

123. Lee, B.; Park, J.-H.; Byun, J.-Y.; Kim, J. H.; Kim, M.-G., Biosens. Bioelectron.

2018, 102, 504-509.

124. Chrouda, A.; Sbartai, A.; Baraket, A.; Renaud, L.; Maaref, A.; Jaffrezic-Renault, N., Anal. Biochem. 2015, 488, 36-44.

125. Orlov, A. V.; Burenin, A. G.; Massarskaya, N. G.; Betin, A. V.; Nikitin, M. P.;

Nikitin, P. I., Sens. Actuators, B 2017, 246, 1080-1084.

126. Armbruster, D. A.; Pry, T., Clin. Biochem. Rev. 2008, 29 (Suppl 1), S49-S52.

127. Kolb Hartmuth, C.; Finn, M. G.; Sharpless, K. B., Angew. Chem. Int. Ed. 2001, 40

(11), 2004-2021.

128. Kolb, H. C.; Sharpless, K. B., Drug Discov. Today 2003, 8 (24), 1128-1137.

129. Hein, C. D.; Liu, X.-M.; Wang, D., Pharm. Res. 2008, 25 (10), 2216-2230.

130. Becer, C. R.; Hoogenboom, R.; Schubert Ulrich, S., Angew. Chem. Int. Ed. 2009, 48 (27), 4900-4908.

131. Nicolaou, K. C.; Snyder Scott, A.; Montagnon, T.; Vassilikogiannakis, G., Angew.

Chem. Int. Ed. 2002, 41 (10), 1668-1698.

132. Lutz, J.-F., Angew. Chem. Int. Ed. 2007, 46 (7), 1018-1025.

133. Agard, N. J.; Prescher, J. A.; Bertozzi, C. R., J. Am. Chem. Soc. 2004, 126 (46), 15046-15047.

134. Hein, J. E.; Fokin, V. V., Chem. Soc. Rev. 2010, 39 (4), 1302-1315.

135. Huisgen, R., Angew. Chem. Int. Ed. 1963, 2 (10), 565-598.

136. Meldal, M.; Tornøe, C. W., Chem. Rev. 2008, 108 (8), 2952-3015.

137. Tornøe, C. W.; Christensen, C.; Meldal, M., J. Org. Chem. 2002, 67 (9), 3057-3064.

138. Rostovtsev Vsevolod, V.; Green Luke, G.; Fokin Valery, V.; Sharpless, K. B., Angew. Chem. Int. Ed. 2002, 41 (14), 2596-2599.

139. Bock Victoria, D.; Hiemstra, H.; van Maarseveen Jan, H., Eur. J. Org. Chem. 2005, 2006 (1), 51-68.

140. Himo, F.; Lovell, T.; Hilgraf, R.; Rostovtsev, V. V.; Noodleman, L.; Sharpless, K.

B.; Fokin, V. V., J. Am. Chem. Soc. 2005, 127 (1), 210-216.

141. Rodionov Valentin, O.; Fokin Valery, V.; Finn, M. G., Angew. Chem. Int. Ed. 2005, 44 (15), 2210-2215.

142. Chan, T. R.; Hilgraf, R.; Sharpless, K. B.; Fokin, V. V., Org. Lett. 2004, 6 (17), 2853-2855.

143. Sletten Ellen, M.; Bertozzi Carolyn, R., Angew. Chem. Int. Ed. 2009, 48 (38), 6974-6998.

144. Agard, N. J.; Baskin, J. M.; Prescher, J. A.; Lo, A.; Bertozzi, C. R., ACS Chem. Biol. 
2006, 1 (10), 644-648.

145. Ess, D. H.; Jones, G. O.; Houk, K. N., Org. Lett. 2008, 10 (8), 1633-1636.

146. Johnson, J. A.; Baskin, J. M.; Bertozzi, C. R.; Koberstein, J. T.; Turro, N. J., Chem. Commun. 2008, (26), 3064-3066.

147. Ning, X.; Guo, J.; Wolfert Margreet, A.; Boons, G.-J., Angew. Chem. Int. Ed. 2008, 47 (12), 2253-2255.

148. Jewett, J. C.; Sletten, E. M.; Bertozzi, C. R., J. Am. Chem. Soc. 2010, 132 (11), 3688-3690.

149. Sharma, A. K.; Heemstra, J. M., J. Am. Chem. Soc. 2011, 133 (32), 12426-12429.

150. Le Droumaguet, C.; Wang, C.; Wang, Q., Chem. Soc. Rev. 2010, 39 (4), 1233-1239.

151. Zhou, Z.; Fahrni, C. J., J. Am. Chem. Soc. 2004, 126 (29), 8862-8863.

152. Seixas de Melo, J. S.; Becker, R. S.; Macanita, A. L., J. Phys. Chem. 1994, 98 (24), 6054-6058.

153. Sivakumar, K.; Xie, F.; Cash, B. M.; Long, S.; Barnhill, H. N.; Wang, Q., Org. Lett. 2004, $6(24), 4603-4606$.

154. Sawa, M.; Hsu, T.-L.; Itoh, T.; Sugiyama, M.; Hanson, S. R.; Vogt, P. K.; Wong, C.H., Proc. Natl. Acad. Sci. U.S.A. 2006, 103 (33), 12371.

155. Sun, H.; Peng, X., Bioconjugate Chem. 2013, 24 (7), 1226-1234.

156. McKay, C. S.; Finn, M. G., Chem. Biol. 2014, 21 (9), 1075-1101.

157. Spiropulos, N. G.; Heemstra, J. M., Artif DNA PNA XNA 2012, 3 (3), 123-128.

158. Tyndall, S.; Wong, K. F.; VanAlstine-Parris, M. A., J. Org. Chem. 2015, 80 (18), 8951-8953.

159. Von Pechmann, H.; Duisberg, C., Ber. Dtsch. Chem. Ges. 1883, 16 (2), 2119-2128.

160. Fan, W.; Tong, X.; Yan, Q.; Fu, S.; Zhao, Y., Chem. Commun. 2014, 50 (88), $13492-13494$.

161. Chen, Y.; Wang, S.; Xu, X.; Liu, X.; Yu, M.; Zhao, S.; Liu, S.; Qiu, Y.; Zhang, T.;

Liu, B.-F.; Zhang, G., J. Med. Chem. 2013, 56 (11), 4671-4690.

162. Krishnaswamy, G.; Desai, N. R.; Naika, R. N. H.; Mahadevan, K. M.; Satyendra, R.

V.; Kumar, D. B. A., J. Chem. Pharm. Res. 2016, 8 (4), 19-28.

163. Murthy, P. K.; Krishnaswamy, G.; Armaković, S.; Armaković, S. J.; Suchetan, P.

A.; Desai, N. R.; Suneetha, V.; SreenivasaRao, R.; Bhargavi, G.; Aruna Kumar, D. B., J. Mol. Struct. 2018, 1162, 81-95.

164. Ji, W.; Liu, G.; Wang, F.; Zhu, Z.; Feng, C., Chem. Commun. 2016, 52 (85), 1257412577.

165. Goud, E. Y.; Rao, Y. J.; Hemasri, Y.; Thirupathi, G., Russ. J. Gen. Chem. 2016, 86 (12), 2732-2736.

166. Pedersen, D. S.; Rosenbohm, C., Synthesis 2001, 2001 (16), 2431-2434.

167. Chinchilla, R.; Nájera, C., Chem. Soc. Rev. 2011, 40 (10), 5084-5121.

168. Chinchilla, R.; Nájera, C., Chem. Rev. 2007, 107 (3), 874-922.

169. Sonogashira, K., J. Organomet. Chem. 2002, 653 (1), 46-49.

170. Jeon, M.-K.; Kang, M.-K.; Park, K. H., Tetrahedron 2012, 68 (30), 6038-6053.

171. DeForest, C. A.; Anseth, K. S., Nat. Chem. 2011, 3 (12), 925-931.

172. Huntington J, A., J. Thromb. Haemost. 2005, 3 (8), 1861-1872. 
173. Deng, B.; Lin, Y.; Wang, C.; Li, F.; Wang, Z.; Zhang, H.; Li, X.-F.; Le, X. C., Anal. Chim. Acta 2014, 837, 1-15.

174. Di Cera, E., Mol. Aspects Med. 2008, 29 (4), 203-254.

175. Crawley J. T, B.; Zanardelli, S.; Chion C. K. N, K.; Lane D, A., J. Thromb.

Haemost. 2007, 5 (s1), 95-101.

176. Bock, L. C.; Griffin, L. C.; Latham, J. A.; Vermaas, E. H.; Toole, J. J., Nature 1992, 355, 564.

177. Bock, L. C.; Griffin, L. C.; Latham, J. A.; Vermaas, E. H.; Toole, J. J., Nature 1992, 355 (6360), 564-566.

178. Tasset, D. M.; Kubik, M. F.; Steiner, W., J. Mol. Biol. 1997, 272 (5), 688-698.

179. Tucker, W. O.; Shum, K. T.; Tanner, J. A., Curr. Pharm. Des. 2012, 18 (14), 20142026.

180. Anna, A.; Carme, F.; Maria, T.; Ramon, E., Curr. Pharm. Des. 2012, 18 (14), 20362047.

181. Hamaguchi, N.; Ellington, A.; Stanton, M., Anal. Biochem. 2001, 294 (2), 126-131.

182. Wang, W.; Chen, C.; Qian, M.; Zhao, X. S., Anal. Biochem. 2008, 373 (2), 213-219.

183. Levy, M.; Cater Sean, F.; Ellington Andrew, D., ChemBioChem 2005, 6 (12), 2163 2166.

184. Nicoludis, J. M.; Miller, S. T.; Jeffrey, P. D.; Barrett, S. P.; Rablen, P. R.; Lawton, T. J.; Yatsunyk, L. A., J. Am. Chem. Soc. 2012, 134 (50), 20446-20456.

185. Bell, R.; Stevens, W. K.; Jia, Z.; Samis, J.; Côté, H. C. F.; MacGillivray, R. T. A.; Nesheim, M. E., J. Biol. Chem. 2000, 275 (38), 29513-29520.

186. Chen, A.; Yan, M.; Yang, S., TrAC, Trends Anal. Chem. 2016, 80, 581-593.

187. Stojanovic, M. N.; de Prada, P.; Landry, D. W., J. Am. Chem. Soc. 2000, 122 (46), $11547-11548$.

188. Liu, J.; Bai, W.; Niu, S.; Zhu, C.; Yang, S.; Chen, A., Sci. Rep. 2014, 4, 7571. 189. Liu, X.; Shi, L.; Hua, X.; Huang, Y.; Su, S.; Fan, Q.; Wang, L.; Huang, W., ACS Appl. Mater. Int. 2014, 6 (5), 3406-3412.

190. Du, Y.; Guo, S.; Qin, H.; Dong, S.; Wang, E., Chem. Commun. 2012, 48 (6), 799801.

191. Jiang, H.; Ling, K.; Tao, X.; Zhang, Q., Biosensors Bioelectron. 2015, 70, 299-303. 192. Yuan, B.; Zhou, Y.; Guo, Q.; Wang, K.; Yang, X.; Meng, X.; Wan, J.; Tan, Y.; Huang, Z.; Xie, Q.; Zhao, X., Chem. Commun. (Cambridge, U. K.) 2016, 52 (8), 15901593.

193. Lei, Y.; Tang, J.; Shi, H.; Ye, X.; He, X.; Xu, F.; Yan, L. a.; Qiao, Z.; Wang, K., Anal. Chem. (Washington, DC, U. S.) 2016, 88 (23), 11699-11706.

194. Liu, X.; Li, X.; Lu, Y.; Cao, J.; Li, F., Anal. Methods 2018, 10 (19), 2281-2286. 195. Lin, Z.; Chen, L.; Zhu, X.; Qiu, B.; Chen, G., Chem. Commun. (Cambridge, U. K.) 2010, 46 (30), 5563-5565.

196. Chen, J.; Zhang, J.; Li, J.; Yang, H.-H.; Fu, F.; Chen, G., Biosens. Bioelectron. 2010, 25 (5), 996-1000.

197. Wang, Q.; Huang, J.; Yang, X.; Wang, K.; He, L.; Li, X.; Xue, C., Sens. Actuators, B 2011, 156 (2), 893-898. 
198. Sergelen, K.; Liedberg, B.; Knoll, W.; Dostalek, J., Analyst 2017, 142 (16), 2995 3001.

199. Sharma, A. K.; Kent, A. D.; Heemstra, J. M., Anal. Chem. 2012, 84 (14), $6104-$ 6109.

200. Sun, N.; Guo, Q.; Shao, J.; Qiu, B.; Lin, Z.; Wong, K. Y.; Chen, G., Anal. Methods 2014, $6(10), 3370-3374$.

201. Xu, X.; Zhang, J.; Yang, F.; Yang, X., Chem. Commun. 2011, 47 (33), 9435-9437. 202. Liu, X.; Shi, L.; Hua, X.; Huang, Y.; Su, S.; Fan, Q.; Wang, L.; Huang, W., ACS Appl. Mater. Interfaces 2014, 6 (5), 3406-3412.

203. Stojanovic, M. N.; de Prada, P.; Landry, D. W., J. Am. Chem. Soc. 2001, 123 (21), 4928-4931.

204. Stojanovic, M. N.; Landry, D. W., J. Am. Chem. Soc. 2002, 124 (33), 9678-9679.

205. Neves, M. A. D.; Reinstein, O.; Saad, M.; Johnson, P. E., Biophys. Chem. 2010, 153 (1), 9-16.

206. Neves, M. A. D.; Reinstein, O.; Johnson, P. E., Biochemistry 2010, 49 (39), 84788487.

207. Slavkovic, S.; Altunisik, M.; Reinstein, O.; Johnson, P. E., Bioorg. Med. Chem. 2015, 23 (10), 2593-2597.

208. Zhang, J.; Wang, L.; Pan, D.; Song, S.; Boey Freddy, Y. C.; Zhang, H.; Fan, C., Small 2008, 4 (8), 1196-1200.

209. Cai, Q.; Chen, L.; Luo, F.; Qiu, B.; Lin, Z.; Chen, G., Anal. Bioanal. Chem. 2011, 400 (1), 289-294.

210. Tang, Y.; Long, F.; Gu, C.; Wang, C.; Han, S.; He, M., Anal. Chim. Acta 2016, 933, 182-188.

211. Yu, H.; Canoura, J.; Guntupalli, B.; Lou, X.; Xiao, Y., Chem. Sci. 2017, 8 (1), 131141.

212. Yu, H.; Canoura, J.; Guntupalli, B.; Alkhamis, O.; Xiao, Y., Anal. Chem. 2018, 90 (3), 1748-1758.

213. Drake, L. R.; Scott, P. J. H., ACS Chem. Neurosci. 2018.

214. Carroll, F. I.; Lewin, A. H.; Boja, J. W.; Kuhar, M. J., J. Med. Chem. 1992, 35 (6), 969-981.

215. Biondich, A. S.; Joslin, J. D., Wilderness Environ. Med. 2015, 26 (4), 567-571.

216. Goldberg, M. F., Arch. Ophthalmol. 1984, 102 (10), 1443-1447.

217. Koller, C., Arch. Ophthalmol. 1934, 12 (4), 473-474.

218. O'Leary, M. E.; Hancox, J. C., Br. J. Clin. Pharmacol. 2010, 69 (5), 427-442.

219. Ritz, M. C.; Cone, E. J.; Kuhar, M. J., Life Sci. 1990, 46 (9), 635-645.

220. Rump, A. F. E.; Theisohn, M.; Klaus, W., Forensic Sci. Int. 1995, 71 (2), 103-115.

221. Sun, H.; Peng, X., Bioconj. Chem. 2013, 24 (7), 1226-1234.

222. Rotaru, A.; Dutta, S.; Jentzsch, E.; Gothelf, K.; Mokhir, A., Angew. Chem. Int. Ed. 2010, 49 (33), 5665-5667.

223. Zhou, Z.; Du, Y.; Dong, S., Anal. Chem. 2011, 83 (13), 5122-5127.

224. Ma, S.-Y.; Yeh, Y.-C., Anal. Methods 2015, 7 (16), 6475-6478.

225. Chen, J.; Liu, J.; Fang, Z.; Zeng, L., Chem. Commun. 2012, 48 (7), 1057-1059. 
226. Qiu, L.; Zhou, H.; Zhu, W.; Qiu, L.; Jiang, J.; Shen, G.; Yu, R., New J. Chem. 2013, 37 (12), 3998-4003.

227. Oueslati, R.; Cheng, C.; Wu, J.; Chen, J., Biosens. Bioelectron. 2018, 108, 103-108. 228. Reinstein, O.; Yoo, M.; Han, C.; Palmo, T.; Beckham, S. A.; Wilce, M. C. J.; Johnson, P. E., Biochemistry 2013, 52 (48), 8652-8662. 